UNIVERSITY OF LJUBLJANA

FACULTY OF ECONOMICS

SHAOFANG LI

FINANCIAL INSTITUTIONS IN THE GLOBAL FINANCIAL CRISIS: THE ROLE OF FINANCIAL DERIVATIVES, BANK CAPITAL, AND CLEARING AND CUSTODY SERVICES

DOCTORAL DISSERTATION 


\section{AUTHORSHIP STATEMENT}

The undersigned Shaofang Li, a student at the University of Ljubljana, Faculty of Economics, (hereafter: FELU), declare that I am the author of the doctoral dissertation entitled Financial Institutions in the Global Financial Crisis: The Role of Financial Derivatives, Bank Capital, and Clearing and Custody Services, written under supervision of Assistant Professor Matej Marinč, PhD.

In accordance with the Copyright and Related Rights Act (Official Gazette of the Republic of Slovenia, Nr. 21/1995 with changes and amendments) I allow the text of my doctoral dissertation to be published on the FELU website.

I further declare

- the text of my doctoral dissertation to be based on the results of my own research;

- the text of my doctoral dissertation to be language-edited and technically in adherence with the FELU's Technical Guidelines for Written Works which means that I

- cited and / or quoted works and opinions of other authors in my doctoral dissertation in accordance with the FELU's Technical Guidelines for Written Works and

o obtained (and referred to in my doctoral dissertation) all the necessary permits to use the works of other authors which are entirely (in written or graphical form) used in my text;

- to be aware of the fact that plagiarism (in written or graphical form) is a criminal offence and can be prosecuted in accordance with the Copyright and Related Rights Act (Official Gazette of the Republic of Slovenia, Nr. 21/1995 with changes and amendments);

- to be aware of the consequences a proven plagiarism charge based on the submitted doctoral dissertation could have for my status at the FELU in accordance with the relevant FELU Rules on Doctoral Dissertation.

Date of public defense:

Committee Chair:

Supervisor:

Co-supervisor:

Member:

Ljubljana,

(Month in words / Day / Year)
Author's signature: 


\section{Financial Institutions in the Global Financial Crisis: The Role of Financial Derivatives, Bank Capital, and Clearing and Custody Services \\ Summary}

The research purpose of this dissertation is to study the role of financial institutions in the financial markets in normal times and during the global financial crisis. The global financial crisis forced a large number of financial institutions to restructure their core operations, rely substantially on the government support, or even enter insolvency procedures. This raises concerns about solvency and liquidity of financial institutions and indicates that special attention should be devoted to analyzing the impact of the financial crisis on financial institutions. Such analysis could substantially improve the responses of regulators, policy makers, and financial institutions themselves to the adverse events in the future. To that end, this doctoral dissertation evaluates the role of financial derivatives, bank capital, and clearing and settlement services in normal times and during the global financial crisis.

By using the most recent data of U.S. bank holding companies, Chapter 1 examines the impact of financial derivatives on risk exposures of BHCs in normal times and during the global financial crisis. The empirical analysis employs a two-stage time-series cross-section regression model to examine the relationship between risk exposures and the use of financial derivatives. In the first stage, the stock return of each BHC is regressed against the changes in the market return, interest rate, exchange rate, and credit spread. In this way, risk betas that measure the BHC's systematic (i.e., nondiversifiable) exposure towards market risk, interest rate risk, exchange rate risk, and credit risk can be obtained. In the second-stage regression, the risk betas are regressed against the on-balance-sheet variables and financial derivatives variables. The results show that financial derivatives are positively and significantly related to systematic risk exposures of BHCs. Higher use of interest rate derivatives, exchange rate derivatives, and credit derivatives corresponds to greater systematic interest rate risk, exchange rate risk, and credit risk. The positive relationship strengthens with the size of a BHC. Our analysis also confirms a positive relationship between trading derivatives and systematic risks, as well as between hedging derivatives and systematic risks.

Chapter 2 aims to investigate whether and how different types of bank capital affect bank lending and whether this relation changes in times of the global financial crisis compared to normal times. This chapter focuses on the decline in credit growth due to the 2008-2010 global financial crisis and also studies the impact of other variables on lending growth in normal times and in the times of a financial crisis. Also of an interest is how the level of deposits affects lending growth and the decline in lending in the times of a financial crisis. The impact of the size of a bank on credit growth is also studied. Several other dimensions that may influence the decline in credit growth during the financial crisis are also analyzed. The analysis shows a significant and positive effect of the tier 1 capital ratio on bank loan growth during the global financial crisis. This positive effect seems to be more pronounced for small banks and for banks in the non-OECD and BRIC countries. Customer deposits also positively affected bank lending during the global financial crisis. Furthermore, the results also indicate some (but weak) evidence that the tier 2 capital ratio and interbank deposits positively affect loan growth in 
normal times and that interbank deposits negatively affected bank lending during the global financial crisis. The evidence highlights a sharp contrast on the impact of different funding sources on bank lending during the global financial crisis. Whereas tier 1 capital and customer deposits acted as a stable source of funding during the global financial crisis, tier 2 capital and interbank deposits spurred bank lending during normal times but did not do so during the global financial crisis. During normal times, a bank lends more if the tier 1 capital ratio of competing banks is high. This relationship reversed during the global financial crisis: during the global financial crisis, a bank lent more if the tier 1 capital ratio of competing banks was low.

Chapter 3 aims to contribute to the scarce knowledge of competitive landscape in the clearing and settlement industry. This chapter uses the unbalanced annual data of 49 clearing and settlement institutions from 24 countries during 1989-2012, employs the Panzar and Rosse (1982, 1987) model, Lerner index (1934), and Boone indicator (2001, 2008), and examines the competitive conditions in the clearing and settlement industry. The findings suggest that monopoly equilibrium exists in the clearing and settlement industry. During the global financial crisis, the level of competition between clearing and settlement institutions is higher than in normal times. International CSDs face higher competition than CSDs in the local market. Our evidence also indicates that competition increases continuously over time, possibly due to the technological development and implementation of new clearing and settlement systems. The results reveal that competition increases with the size of clearing and settlement institutions and after mergers and acquisitions between clearing and settlement institutions. Our findings also suggest that competition between clearing and settlement institutions in the U.S. market is higher than in the European market. This indicates that renewed initiative is necessary to enhance competition between clearing and settlement institutions in Europe.

Keywords: Financial Derivatives, Interest Rate Derivatives, Exchange Rate Derivatives, Credit Derivatives, Systematic Risk, Tier 1 capital, Bank Capital, Bank Lending, Clearing and Settlement Services, Competitive Condition, Panzar-Rosse Model, Global Financial Crisis 


\section{FINANČNE INSTITUCIJE V SVETOVNA FINANČNI KRIZI: VLOGA IZVEDENIH FINANČNIH INSTRUMENTOV, BANČNEGA KAPITALA, TER STORITEV OBRAČUNA IN SKRBNIŠKE \\ Povzetek}

Namen raziskave te disertacije je študija vloge finančnih institucij na finančnih trgih v normalnih razmerah in $v$ času svetovne finančne krize. Slednja je prisilila mnoge finančne institucije $\mathrm{k}$ rekonstruiranju glavnih dejavnosti, $\mathrm{k}$ večjemu zanašanju na vladno podporo ali celo $\mathrm{v}$ postopek insolventnosti, kar pa vzbuja skrb glede solventnosti in likvidnosti finančnih institucij in nakazuje na to, da bi bilo treba posvetiti posebno pozornost analizi vpliva finančne krize na finančne institucije. Taka analiza bi lahko znatno izboljšala odziv regulatorjev, oblikovalcev politike in samih finančnih institucij na neželene dogodke v prihodnje. Proti koncu disertacija oceni vlogo izvedenih finančnih instrumentov, bančnega kapitala ter storitev obračuna in poravnave $\mathrm{v}$ normalnih razmerah in med svetovno finančno krizo.

Prvo poglavje $\mathrm{z}$ uporabo najnovejših podatkov ameriških bančnih holdingov preiskuje vpliv izvedenih finančnih instrumentov na izpostavljenost bančnih holdingov tveganju v normalnih razmerah in med svetovno finančno krizo. Empirična analiza $\mathrm{z}$ uporabo dvostopenjskega, presečnega regresijskega modela in $\mathrm{s}$ pomočjo časovnih vrst proučuje razmerje med izpostavljenostjo tveganju in uporabo izvedenih finančnih instrumentov. V prvi fazi se z metodo regresije izračuna donos delnic vsakega bančnega holdinga proti spremembam v tržnem donosu, obrestni meri, menjalnem tečaju in kreditnem pribitku. Na ta način lahko pridobimo bete tveganja, ki izmerijo sistematično izpostavljenost bančnih holdingov tržnemu tveganju, tveganju obrestne mere ter tečajnemu in kreditnemu tveganju. V drugi fazi pa je uporabljena metoda regresije med betami tveganja in bilančnimi spremenljivkami ter spremenljivkami izvedenih finančnih instrumentov. Rezultati kažejo na to, da izvedeni finančni instrumenti pozitivno in močno vplivajo na izpostavljenost bančnih holdingov sistematičnemu tveganju. Večja uporaba obrestnih, menjalnih in kreditnih izvedenih finančnih instrumentov ustreza večjemu sistematičnemu obrestnemu, menjalnemu in kreditnemu tveganju. Pozitivno razmerje se okrepi z velikostjo holdinga. Naša analiza prav tako potrjuje pozitivno razmerje tako med izvedenimi finančnimi instrumenti za trgovanje in sistematičnim tveganjem, kot tudi med finančnimi instrumenti za varovanje pred tveganjem in sistematičnim tveganjem.

Drugo poglavje preučuje, če in kako različne vrste bančnega kapitala vplivajo na bančna posojila, in če se to razmerje spreminja $\mathrm{v}$ času svetovne finančne krize $\mathrm{v}$ primerjavi $\mathrm{z}$ normalnimi razmerami. Poglavje se osredotoča na upad kreditne rasti zaradi svetovne finančne krize v letih 2008-2010 in prav tako preučuje vpliv drugih spremenljivk na rast posojil v normalnih razmerah in v času krize. Prav tako je zanimivo, kako raven bančnih vlog vpliva na rast in upad posojil med krizo, študija pa vključuje tudi vpliv velikosti bank na kreditno rast. Analizira tudi vpliv drugih razsežnosti, ki lahko vplivajo na upad kreditne rasti med finančno krizo. Analiza kaže na znaten, pozitiven vpliv deleža kapitala prvega reda (Tier 1 kapitala) na rast bančnih posojil med svetovno finančno krizo. Zdi se, da je vpliv bolj izrazit pri manjših bankah in bankah držav nečlanic OECD in držav BRIK, prav tako pa so depoziti strank pozitivno vplivali na rast bančnih posojil med svetovno finančno krizo. Rezultati do neke mere nakazujejo tudi na to, da je delež 
kapitala drugega reda (tier 2 kapitala) skupaj z medbančnimi vlogami pozitivno vplival na rast bančnih posojil v normalnih razmerah, in da so medbančne vloge negativno vplivale na bančna posojila $\mathrm{v}$ času krize. Dokazi opozarjajo na ostra nasprotja med vplivi različnih virov financiranja na bančna posojila $v$ času finančne krize. Medtem ko so kapital prvega reda in depoziti strank predstavljali stabilen vir financiranja med svetovno finančno krizo, pa je kapital drugega reda skupaj z medbančnimi vlogami spodbudil bančna posojila v normalnih razmerah vendar ne tudi v času krize. V normalnih razmerah je bilo bančnih posojil več, če je bil delež kapitala prvega reda konkurenčnih bank visok, v času svetovne finančne krize pa so banke dajale več posojil, če je bil delež kapitala prvega reda konkurenčnih bank nizek.

Tretje poglavje skuša prispevati $\mathrm{k}$ do sedaj še omejenemu znanju o konkurenčnem okolju v industriji obračuna in poravnave. Poglavje uporablja neuravnotežene letne podatke 49 institucij obračuna in poravnave iz 24 držav med leti 1989-2012 in s pomočjo modela Panzar-Rosse (1982, 1987), Lernerjevega indeksa (1934) in Boonovega indikatorja (2001, 2008) preučuje konkurenčne pogoje $\mathrm{v}$ industriji obračuna in poravnave. Ugotovitve kažejo na obstoj monopolnega ravnovesja $v$ le-tej industriji. Med svetovno finančno krizo je raven konkurence med institucijami obračuna in poravnave višja kot v normalnih razmerah. Mednarodne centralne depotne družbe (CDD) se soočajo z večjo konkurenco kot CDD na lokalnih trgih. Naši dokazi prav tako nakazujejo na to, da se konkurenca skozi čas nenehno povečuje, kar je mogoče pripisati tehnološkemu napredku in izvajanju novih sistemov obračuna in poravnave. Naši rezultati tudi razkrivajo, da je konkurenca med institucijami obračuna in poravnave večja na ameriškem trgu kot na evropskem, kar pa kaže na to, da bo potrebno obnoviti pobudo za povečanje konkurence med evropskimi institucijami obračuna in poravnave.

Ključne besede: Izvedeni Finančni Instrumenti, Obrestni Izvedeni Finančni Instrumenti, Tečajni Izvedeni Finančni Instrumenti, Kreditni Izvedeni Finančni Instrumenti, Sistematično Tveganje, Kapital Prvega Reda, Bančni Kapital, Bančna Posojila, Storitve Obračuna in Poravnave, Konkurenčni Pogoji, Model Panzar-Rosse, Svetovna Finančna Kriza 


\section{TABLE OF CONTENTS}

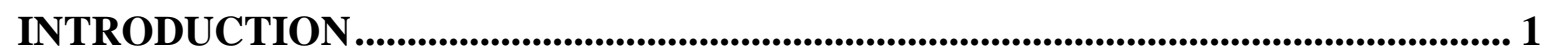

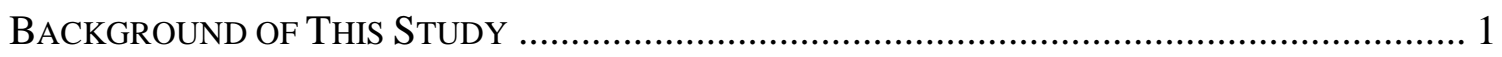

RESEARCH QUESTIONS ADDRESSED IN THIS STUDY ...................................................... 2

STRUCTURE AND CONTENTS OF THIS STUDY .............................................................. 4

\section{THE USE OF FINANCIAL DERIVATIVES AND RISKS OF U.S. BANK HOLDING}

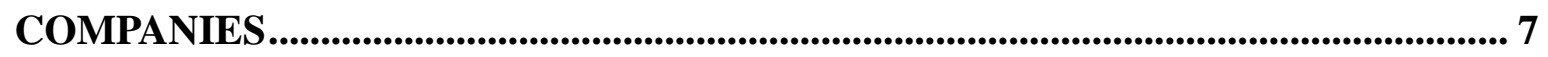

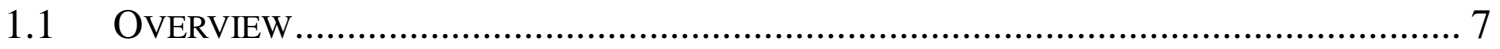

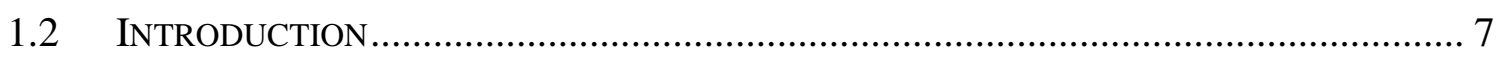

1.3 RELATION TO THE LITERATURE AND HYPOTHESIS FORMATION............................... 10

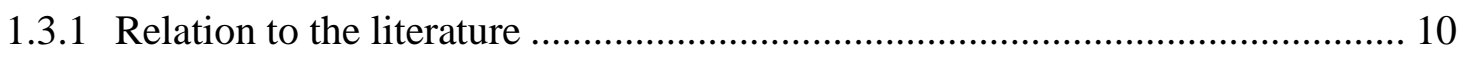

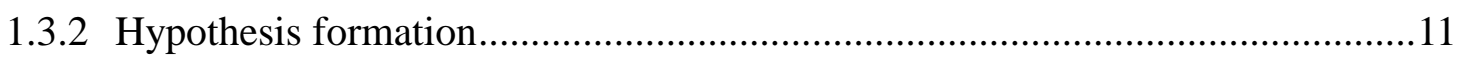

1.4 Data Sources, SAMPLE SELECTION AND Data DESCRIPTION................................ 14

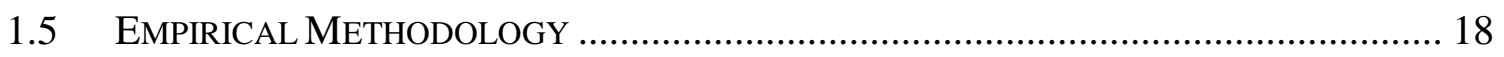

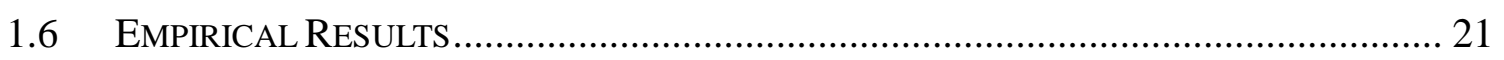

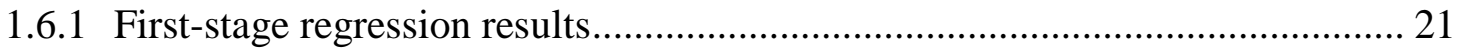

1.6.2 Bank-specific determinants of risk betas: initial analysis.................................. 26

1.6.3 Purposes of financial derivatives and systematic risk exposures ...................... 33

1.6.4 The global financial crisis and financial derivatives ..................................... 39

1.6.5 The impact of financial derivatives on market risk and idiosyncratic risk ........ 41

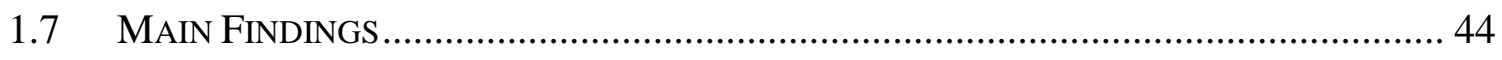

2 QUALITY OF BANK CAPITAL AND BANK LENDING BEHAVIOR DURING THE GLOBAL FINANCIAL CRISIS ............................................................................ 45

2.1 OVERVIEW

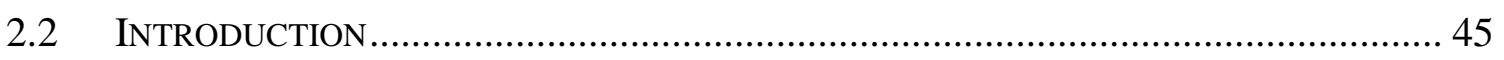

2.3 PreVIOUS StUdiES AND DEVELOPMENT OF HyPOTHESES ….................................. 47

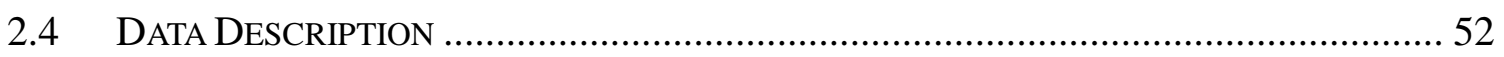

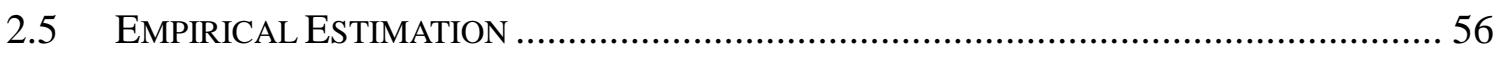

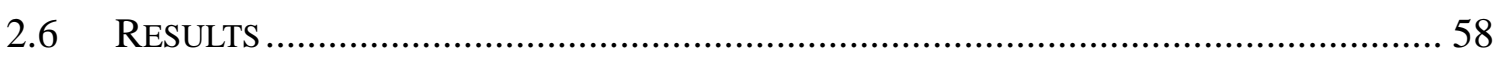

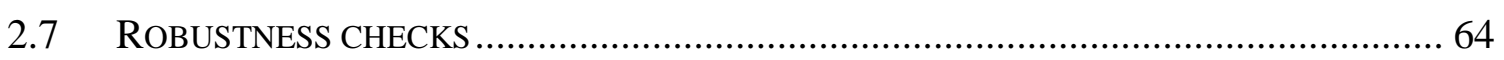

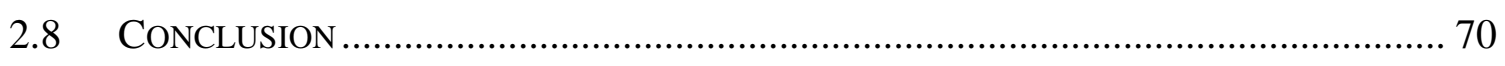

3 COMPETITION IN THE CLEARING AND SETTLEMENT INDUSTRY ....... 71

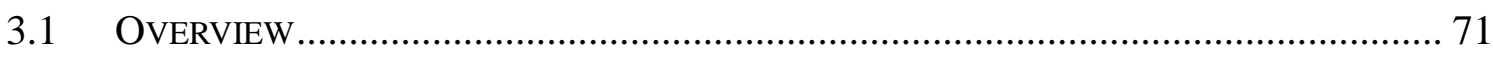

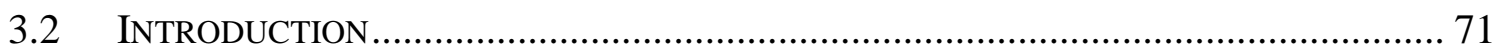

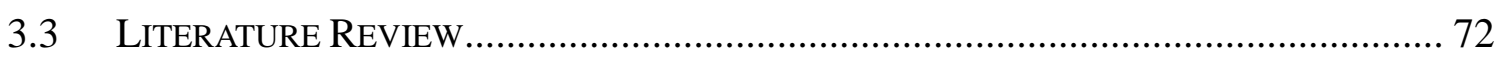

3.3.1 Clearing and settlement institutions in Europe and in the U.S. ....................... 72

3.3.2 Industry structure in clearing and settlement services ..................................... 74

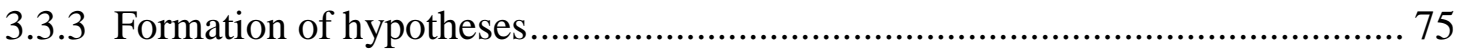


3.4 Methodological Basis to MeAsuring CoMPETITION ......................................77

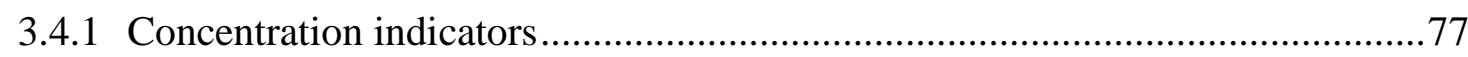

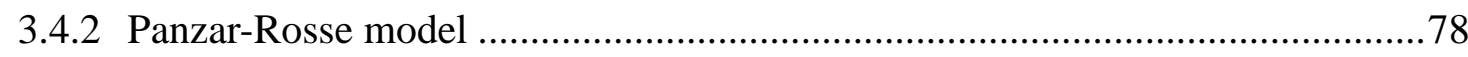

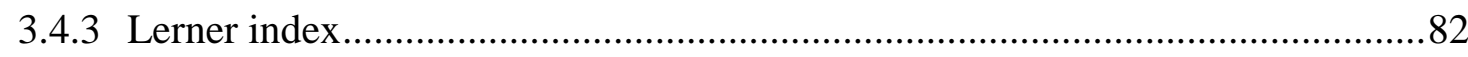

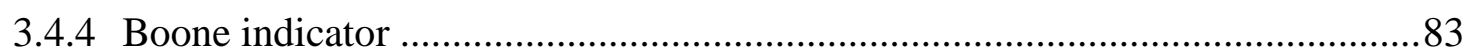

3.5 DESCRIPTION OF DATA AND CONCENTRATION MEASURES ........................................8

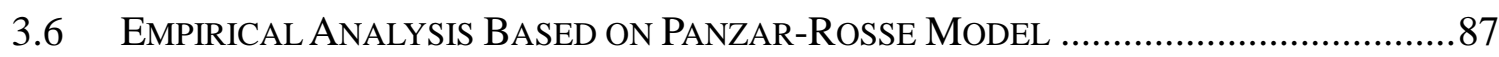

3.7 FACTORS AFFECTING THE LERNER INDEX …....................................................... 93

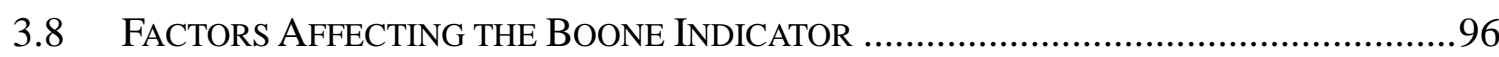

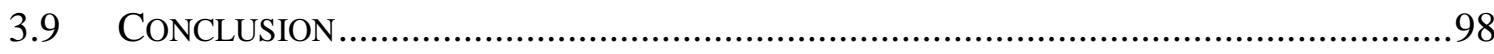

GENERAL DISCUSSION AND CONCLUSION ........................................................99

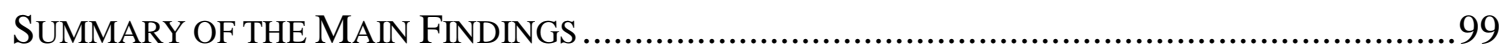

OVERARCHING THEORETICAL AND EMPIRICAL CONTRIBUTIONS ................................... 100

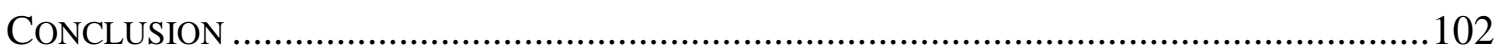

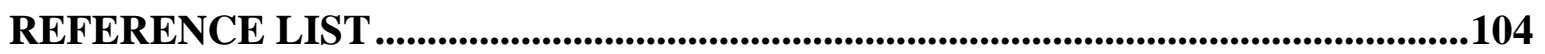

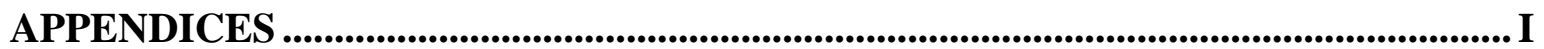




\section{LIST OF TABLES}

Table 1.1. Description of Variables

Table 1.2. Financial Derivatives Used by U.S. BHCs (Notional Principal Amounts, \$ billion)

Table 1.3. Difference in Means: Financial Characteristics of BHCs, Large BHCs, and Small

$\mathrm{BHCs}$

Table 1.4. Correlation Coefficients Between Macroeconomic Factors ................................. 21

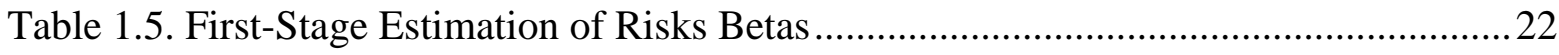

Table 1.6. Difference between Risk Betas: Before and After Use of Financial Derivatives . 22

Table 1.7. Correlation Between On-and Off-balance-Sheet BHCs' Specific Variables ......... 28

Table 1.8. Determinants of Interest Rate, Exchange Rate, and Credit Risk Betas

Table 1.9. Determinants of Interest Rate, Exchange Rate, and Credit Risk Betas, and Interaction Terms

Table 1.10. Impact of Financial Crisis on the Determinants of Interest Rate, Exchange Rate, and Credit Risk Betas

Table 1.11. The Impact of Financial Derivatives on Scaled Idiosyncratic Risk $\left(1-\mathrm{R}^{2}\right) \ldots \ldots . .42$

Table 1.12. The Impact of Financial Derivatives on Market Risk Beta...............................43

Table 2.1. Descriptive Statistics of the Total Sample of Banks, 2000-2010........................54

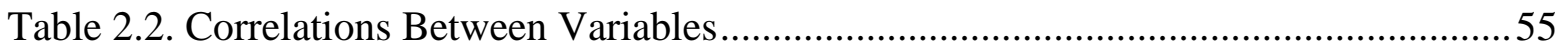

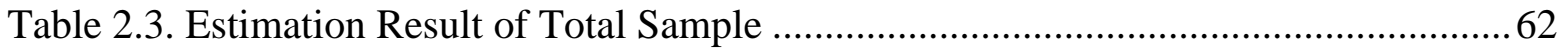

Table 2.4. Estimation Result of Subsamples by Different Regions ......................................66

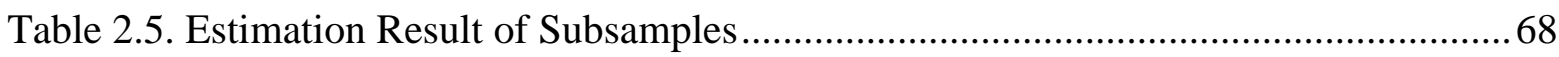

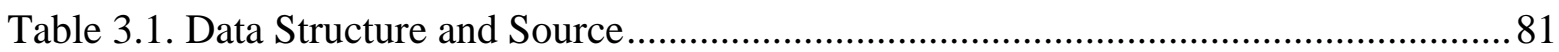

Table 3.2. Summary of Sample Clearing and Settlement Institutions, 1989-2012 ............... 85

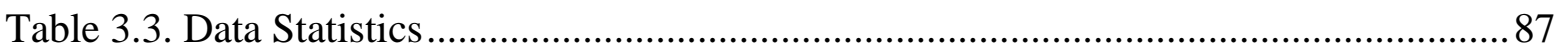

Table 3.4. The Competitive Equilibrium and the Impact of the Global Financial Crisis on

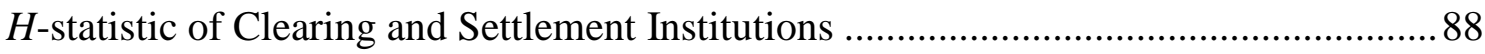

Table 3.5. The Impact of Institutional Structure (ICSD) on $H$-statistic of Clearing and

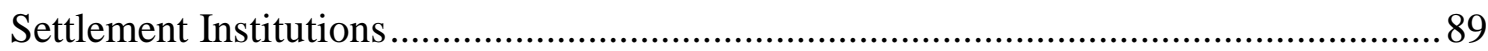

Table 3.6. The Impact of Size on $H$-statistic of Clearing and Settlement Institutions ...........91

Table 3.7. The Impact of Merger on $H$-statistic of Clearing and Settlement Institutions ...... 91

Table 3.8. The Impact of ICT Ratio on $H$-statistic of Clearing and Settlement Institutions .. 92 Table 3.9. The Impact of Geographic Location (USregion) on $H$-statistic of Clearing and

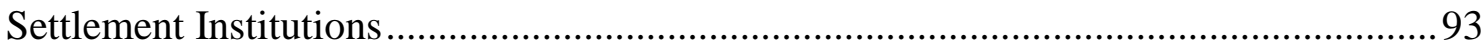

Table 3.10. Estimation of Factors Affecting the Lerner Index ........................................... 95

Table 3.11. Estimation of Factors Affecting the Boone Indicator .....................................97

Table 4.1. Summary of Main Findings and Contributions in Chapters 1, 2, and 3............. 101 


\section{LIST OF FIGURES}

Figure 1.1. Financial Derivatives Held by Large BHCs (\$ Trillion) …................................. 8

Figure 1.2. Financial Derivatives Held by Small BHCs (\$ Trillion) .................................... 8

Figure 1.3. Financial Derivatives Held for Trading Purposes ( $\$$ Trillion) ............................. 9

Figure 1.4. Financial Derivatives Held for Hedging Purposes (\$ Trillion) ........................... 9

Figure 1.5. Interest Rate Risk Sensitivity for Three Separate Sample Groups ...................... 23

Figure 1.6. Exchange Rate Risk Sensitivity for Three Separate Sample Groups .................. 24

Figure 1.7. Credit Risk Sensitivity for Three Separate Sample Groups ................................. 24

Figure 1.8. Market Risk Sensitivity for Three Separate Sample Groups .............................. 24

Figure 1.9. Idiosyncratic Risk $\left(1-\mathrm{R}^{2}\right.$ ) Sensitivity for Three Separate Sample Groups ........ 25

Figure 1.10. Interest Rate Risk Sensitivity by Different Size of BHCs (by Interest Rate

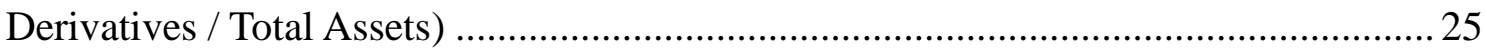

Figure 1.11. Exchange Rate Risk Sensitivity by Different Size of BHCs (by Exchange Rate

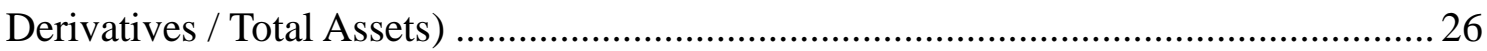

Figure 1.12. Credit Risk Sensitivity by Different Size of BHCs (by Credit Derivatives / Total

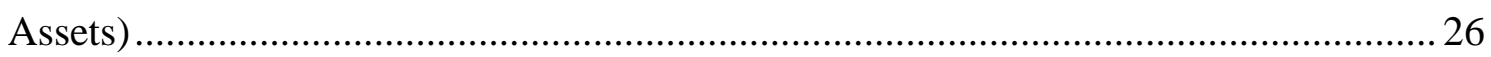

Figure 2.1. Loan Growth of Different Subsamples by Tier 1 ......................................... 46 


\section{INTRODUCTION}

\section{Background of This Study}

Financial institutions are special as they provide a variety of financial services to individuals and other institutions, and the influence of financial institutions is fundamental to the development of economy. The purpose of this dissertation is to study the role of financial institutions in the financial markets in normal times and during the global financial crisis. The global financial crisis, also known as the subprime crisis or the credit crisis, originated in the U.S. subprime mortgage market and spread to other financial markets and countries, and is considered to be the worst financial crisis since the Great Depression in 1930s. The global financial crisis led to a huge loss of economic output and financial wealth. Atkinson, Luttrell, and Rosenblum (2013) indicate that the financial crisis led to the loss of around $40 \%$ to $90 \%$ of one year output of the U.S., an estimated $\$ 6$ trillion to $\$ 14$ trillion, which equals $\$ 50,000$ to $\$ 120,000$ for each U.S. household. ${ }^{1}$ Thakor (2014) estimates that the loss of the total U.S. wealth from the crisis, including human capital and the present value of future wage income, is as high as $\$ 15$ trillion to $\$ 30$ trillion, which is $100 \%-190 \%$ of the 2007 U.S. output.

The crisis also brought the U.S. and the global financial system to the brink of collapse. As a consequence, this forced governments to provide a back stop through wide programs of state aid, restructuring programs and assisted mergers. For example, Bear Stearns, which was the sixth-largest investment bank in the U.S., was bought by JP Morgan Chase in March 2008. Meanwhile, Wells Fargo acquired Wachovia, Washington Mutual and many other small financial institutions that all failed as a consequence of the losses related to the subprime crisis. The consequences revealed to be even worse when Lehman Brothers filed for bankruptcy, Fannie Mae and Freddie Mac, which are government-sponsored enterprises and the key players in the securitization market, and AIG, were bailed out by the government. At the same time, many other large financial institutions were exposed to huge losses because of the use of mortgage-backed securities products. ${ }^{2}$ The subprime crisis forced a large number of financial institutions to restructure their core operations, rely substantially on the government support, or even enter insolvency procedures. This raises the concerns about solvency and liquidity of financial institutions and indicates that special attention should be devoted to the impact of the financial crisis on financial institutions' activities. Such knowledge could substantially improve the responses of regulators, policy makers and financial institutions themselves to the adverse events in the future.

Given the importance of financial institutions and the impact of recent subprime crisis on their behavior, the primary objective of this doctoral dissertation is to evaluate the role of financial derivatives, bank capital, and clearing and settlement services in normal times and during the global financial crisis. To that end, this dissertation proceeds from three perspectives:

\footnotetext{
1 The real GDP for 2007 was $\$ 15.2$ trillion in 2012 dollars (Atkinson, et al., 2013).

2 Source: Deutsche Bank, “Global Markets Research,” March 11, 2008 -
} 
1) explores the relationship between the risk exposures and the use of financial derivatives by bank holding companies (BHCs), and tests the difference between the impact of financial derivatives held for trading and financial derivatives held for hedging on risk exposures;

2) analyzes the effects of bank capital structure on bank lending activities, and tests the effect of different funding sources on credit growth;

3) examines the competitive conditions of clearing and settlement institutions, and investigates whether factors, including the global financial crisis, institutional structure, institutional size, mergers, technological development, and geographic location, affect the competitive conditions in the clearing and settlement industry.

\section{Research Questions Addressed in This Study}

This doctoral dissertation is built on three main parts: Chapter 1 focuses on a direct link between the use of financial derivatives and risk exposures of U.S. BHCs. Because of the need of risk management for profitability of financial institutions, the innovative forms of financial instruments have grown rapidly in recent years. Banking is one of the most regulated industries in the U.S., and the rules on bank risk management are an integral part of the regulations. Risk management techniques that reduce return volatility are generally classified as hedging activities. Financial instruments that increase return volatility are classified as speculative activities. Since March 1995, BHCs are required to report whether their financial derivatives activity is for trading purpose (i.e., mainly for speculative purpose) or for purpose other than trading (i.e., for hedging purpose).

The increased activity in financial derivatives markets was generally looked upon favorably before the global financial crisis. Greenspan (1999) noted that "the value added of derivatives themselves derives from their ability to enhance the process of wealth creation." Trichet (2007) further explained that "[p]rice discovery in the credit derivatives market reduces the risk of mispricing loans." Recently, however, the perspective has turned around as the risks of financial derivatives have become more evident. The Financial Stability Board (2010) concluded that "the crisis demonstrated the potential for contagion arising from the interconnectedness of OTC derivatives market participants and the limited transparency of counterparty relationships." The importance of financial derivatives inspired the first research question in this study, which examines the impact of financial derivatives on systematic risk (systematic interest rate risk, exchange rate risk, and credit risk) of BHCs in normal times and during the global financial crisis. In addition we test whether there is any difference between the impact of financial derivatives held for trading and financial derivatives held for hedging on systematic risk. We employ a dynamic panel analysis on the sample of BHCs in the U.S. in the period between 1997 and 2012.

Chapter 2 focuses on the relation between bank capital structure and bank credit growth in normal times and during the financial crisis. The recent global financial crisis has brought several large financial institutions to the brink of collapse. They obtained government support or have been forced to raise new capital from private investors. The global financial crisis has 
highlighted the importance of bank capital not only for stability in the banking system, but also to increase credit supply in the financial market.

Bank capital needs to be sufficiently high and appropriately structured in order to prevent the future financial crisis (Kashyap et al. (2008), Acharya et al. (2010), and Hart and Zingales (2011)). The regulators pushed for enhanced capital regulation, incorporated in the revised Basel III capital regulatory framework (Basel Committee on Banking Supervision, 2010). As Ben Bernanke, the former chairman of the Federal Reserve, put it, "this framework would require banking organizations to hold more and higher quality capital . . improving the resilience of the U.S. banking system in times of stress, thus contributing to the overall health of the U.S. economy." In Europe, Andrea Enria, the chairman of the European Banking Authority, hailed improved capital positions of European banks by noting that "European banks are now in a stronger position, which should support lending to the real economy ..."4 In contrast, bankers strongly objected to this reasoning. Vikram Pandit, former CEO of Citigroup, argued that "double-digit ratios will undermine lending, slow capital formation, lower demand and restrict growth." 5

While some studies have considered the impact of bank capital on bank lending (e.g. De Haas and Van Lelyveld (2010) and Jiménez et al. (2012)), only a few have empirically examined the relation between the bank capital structure with bank lending. Chapter 2 examines the impact of bank capital and capital structure on bank lending behavior in normal times and during the global financial crisis. Specifically, the main hypothesis is that the higher quality of the bank funding side (i.e., a high tier 1 bank capital ratio, high proportion of customer deposits, and prevalent government support) better supports bank lending during crisis times. This analysis provides new insight into the relation between bank capital structure (and bank funding structure) and bank lending, and contributes to the difference between tier 1 bank capital and tier 2 bank capital.

Chapter 3 focuses on the competitive conditions in the clearing and settlement industry. The globalization and consolidation in capital markets have increased considerably, and trading on international capital markets grows faster. Well-developed clearing and settlement services are the essential ingredients of well-functioning capital markets. As the financial markets become more and more integrated (Boot, 2011), the importance of clearing and settlement services (viewed as a subset of transaction costs that investors face in a banking system (Giddy, Saunders, and Walter, 1996; Schaper, 2008)) is increasing. This indicates that an efficient clearing and settlement system is crucial to minimize the risks and costs involved in the transactions. The progress in financial innovations and standardization of regulatory environments make investors less restricted to their physical market locations. Therefore, clearing and settlement services are faced with increasing competition in domestic and international markets.

\footnotetext{
3 Statement by Chairman Ben S. Bernanke, 7 June, 2012, http://www.federalreserve.gov/newsevents/press/bcreg/bernanke20120607a.htm

${ }_{5}^{4}$ www.eba.europa.eu/News--Communications/Year/2012/Update-implementation-capital-exercise.aspx

5 Vikram Pandit, We must rethink Basel or growth will suffer, Financial Times, 10 November, 2010.
} 
Previous studies show that economies of scale, technological development, cost and revenue efficiency, and mergers and acquisitions affect the performance of stock exchanges and clearing and settlement industry (Hasan and Malkamäki, 2001; Hasan, Malkamäki, and Schmiedel, 2003; Hasan and Schmiedel, 2004; Schmiedel, 2001). Therefore, the focus of Chapter 3 is to examine the competitive conditions in the clearing and settlement industry. We also test whether other factors (i.e., the global financial crisis, institutional structure, institutional size, mergers, technological development, and geographic location) affect competition in the clearing and settlement industry. The empirical analysis covers the clearing and settlement institutions in the U.S. and European market in the sample period from 1989 to 2012.

\section{Structure and Contents of This Study}

Apart from the Introduction and Conclusion, this dissertation is organized in three chapters. Chapter 1 gives an analysis of the relationship between the use of financial derivatives and systematic risk exposures of U.S. bank holding companies. This part tries to answer the following questions: (1) Does the use of financial derivatives have a significant impact on systematic risk exposures of BHCs? (2) Is there any difference between the impact of financial derivatives held for trading and financial derivatives held for hedging? (3) Do the BHCs' size and capital ratio significantly change the relation between the financial derivatives and systematic risk exposures? (4) Does the relation between financial derivatives and systematic risk exposures change during the global financial crisis?

Chapter 1 starts with an overview of previous studies on the theoretical models and empirical studies, followed by research design and sample selection, results of empirical analysis, and ends with discussion and conclusion. In order to extend earlier studies on the relationship between financial derivatives and risk exposures, the research design considers the previous work and studies the joint effect of different risk factors. In this part, the research makes several improvements in research design and sample selection: (1) we use the extended four-factor model that analyzes the joint effect of market risk, interest rate risk, exchange rate risk, and credit risk; (2) we employ the recent sample period (1997-2012) and include a representative sample of BHCs; (3) we take into account the effect of macroeconomic conditions (financial crisis, GDP growth, and income tax rate); (4) we control for the impact of size and capital ratio on systematic risk exposures; and (5) we distinguish between financial derivatives held for trading and financial derivatives held for hedging.

The analysis employs a two-stage time-series cross-section regression model and examines the relationship between systematic risks and the use of financial derivatives. The regression proceeds in two stages (consistent with Fama and French (1992)). In the first stage, the excess stock returns of each $\mathrm{BHC}$ are regressed against changes in the market return, interest rate, exchange rate, and credit spread. In this way, risk betas that measure the BHC's systematic (i.e., nondiversifiable) risk exposures towards market risk, interest rate risk, exchange rate risk, and credit risk can be generated. In the second-stage regression, the risk betas generated in the first stage are regressed against the on-balance-sheet variables and financial derivatives variables. The 
main findings indicate that the use of financial derivatives is positively and significantly related to the BHCs' systematic risk exposures. More specifically, higher use of interest rate derivatives, exchange rate derivatives, and credit derivatives corresponds to greater systematic interest rate risk, exchange rate risk, and credit risk. In addition, the positive relationship between financial derivatives and risks persists for financial derivatives for trading as well as for financial derivatives for hedging.

Chapter 2 provides empirical analysis of the relation between bank capital structure and bank lending in normal times and during the global financial crisis. Financial crises, including the most recent one, have shown that instabilities in banking systems negatively affect real economy. In particular, banks may cut back on lending and this may constrain small and medium businesses and therefore be further detrimental for the economy as a whole. Understanding the determinants of credit growth would enable us to better act in times of a banking crisis or even before the crisis by setting the regulatory standards that would minimize cyclicality of credit growth. The question is whether and, if so, why banks responded differently during the financial crisis 2008-2010 in their credit growth strategies.

To address this question, Chapter 2 first examines whether and how different types of bank capital affect bank lending and whether this relation changed during the 2008-2010 global financial crisis compared to normal times. In addition, the impact of deposits, bank size, state ownership, and implicit of government guarantees on lending growth in normal times and during the times of the global financial crisis are also examined. The results imply that the high quality of bank funding strategy (tier 1 bank capital and retail deposits) and prevalent government backing were crucial to continuous bank lending during the crisis period. The results also indicate that the higher use of tier 2 capital and interbank deposits could be important for increased lending during a normal period, but did not support lending activities during the financial crisis. This chapter concludes by suggesting that in crisis periods high-quality bank capital is a bank's competitive strength.

Chapter 3 focuses on the competitive conditions in the clearing and settlement industry. The exiting literature that investigates the competitive conditions in the clearing and settlement industry is scarce. This chapter employs the Panzar-Rosse model (Panzar and Rosse, 1982, 1987), Lerner index (Lerner, 1934), and Boone indicator (Boone, 2001, 2008), and analyzes the competitive landscape within the clearing and settlement industry. Chapter 3 starts with a description of the role and industry structure in clearing and settlement services, and the measures of competition. It then continues with a research design, including Panzar-Rosse model, the Lerner index, and Boone indicator, followed by the data statistical analysis, and empirical analysis. The chapter analyzes competition in the clearing and settlement industry, and tests how competition is affected by several factors including 1) the global financial crisis; 2) institutional structure; 3) institutional size; 4) mergers; 5) technical development, and 6) geographic location.

The findings under the Panzar-Rosse model, Lerner index, and Boone indicator are consistent. The results show that clearing and settlement institutions operate in monopoly markets. 
International CSDs face higher competition than national CSDs. The level of competition has increased over time, possibly due to the technological development and implementation of new clearing and settlement systems. The results reveal that competition increases with size and after mergers of clearing and settlement institutions. The competition between clearing and settlement institutions in the U.S. market is higher than in the European market.

The rest of the dissertation proceeds as follows: Chapter 1 addresses the relationship between financial derivatives and systematic risks of the U.S. BHCs and is entitled The Use of Financial Derivatives and Risks of U.S. Bank Holding Companies. Chapter 2 analyses the relationship between bank capital and lending and is entitled Quality of Bank Capital and Bank Lending Behavior during the Global Financial Crisis. Chapter 3 investigates competition landscape in clearing and custody services and is entitled Competition in the Clearing and Settlement Industry. Chapter 4 concludes the dissertation. 


\section{THE USE OF FINANCIAL DERIVATIVES AND RISKS OF U.S. BANK HOLDING COMPANIES ${ }^{6}$}

\subsection{Overview}

This chapter examines the impact of financial derivatives on systematic risk of publicly listed U.S. bank holding companies (BHCs) from 1997 to 2012. We find that the use of financial derivatives is positively and significantly related to BHCs' systematic risk exposures. Higher use of interest rate derivatives, exchange rate derivatives, and credit derivatives corresponds to the greater systematic interest rate risk, exchange rate risk, and credit risk. The positive relationship between derivatives and risks persists for derivatives for trading as well as for derivatives for hedging. We also analyze the role of BHCs' size and capital and the impact of the global financial crisis on the relationship between derivatives and risks.

\subsection{Introduction}

Banks have drastically increased the use of financial derivatives in recent decades. The notional principal amount of financial derivatives held by U.S. bank holding companies (BHCs) rose from less than \$18 trillion at the end of 1995 to nearly $\$ 270$ trillion at the end of 2012. Increased activity in financial derivatives markets was generally looked upon favorably before the 2007-2010 global financial crisis. Greenspan (1999) noted that "the value added of derivatives themselves derives from their ability to enhance the process of wealth creation." Trichet (2007) further explained that "[p]rice discovery in the credit derivatives market reduces the risk of mispricing loans." Recently, however, the perspective has turned around because the risks of financial derivatives have become more evident. The Financial Stability Board (2010) concluded that "the crisis demonstrated the potential for contagion arising from the interconnectedness of OTC derivatives market participants and the limited transparency of counterparty relationships." The unanswered question is whether banks use financial derivatives for hedging or for speculative purposes.

This chapter analyzes why BHCs use financial derivatives and, more specifically, whether financial derivatives expose BHCs further towards more or fewer risks. In particular, we measure whether the use of financial derivatives is related to the risk exposures of BHCs towards systematic interest rate risk, exchange rate risk, and credit risk.

We collected on-balance-sheet and off-balance-sheet financial data and stock prices of publicly traded U.S. BHCs from 1997 to 2012. Financial derivatives activity in the U.S. financial market

\footnotetext{
${ }^{6}$ This chapter is co-authored with Matej Marinč. We would like to thank Giuseppe Galloppo, Iftekhar Hasan, Joon Ho Hwang, Marko Košak, Igor Lončarski, Nadia Massoud, Thu Hang Nguyen, and Min-Teh Yu, and the participants at the Midwest Finance Association Conference 2013 in Chicago, the participants at the 9th Conference of Asia-Pacific Association of Derivatives (APAD2013) in Busan, the participants at the AIDEA 2013 Bicentenary conference in Lecce, and the participants at the INFINITI 2014 in Prato for their valuable comments and suggestions. This chapter has been published in International Review of Financial Analysis, 35, 2014, 46-71.

7 FRB of Chicago, holding company data, https://www.chicagofed.org/applications/bhc_data/bhcdata_index.cfm. 
is dominated by a small group of large financial institutions (i.e., the top 25 BHCs hold $99.8 \%$ of the financial derivatives, Office of the Comptroller of the Currency, 2012). We split our sample BHCs into large and small BHCs (asset size more vs. less than $\$ 50$ billion). Figures 1.1 and 1.2 depict the notional principal amounts of interest rate, exchange rate, and credit derivatives held by large BHCs and small BHCs in our sample.

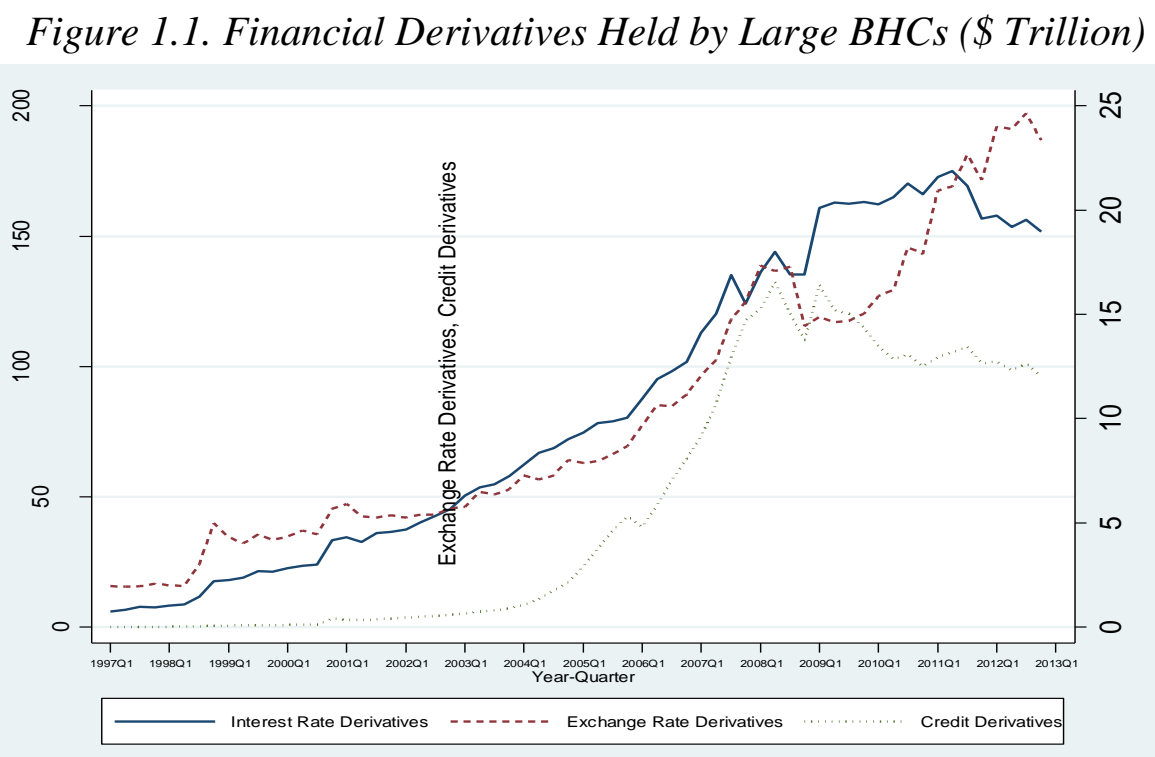

Source: Quarterly data from FR Y-9C, sample period: 1997:Q1-2012:Q4

Figure 1.2. Financial Derivatives Held by Small BHCs (\$ Trillion)

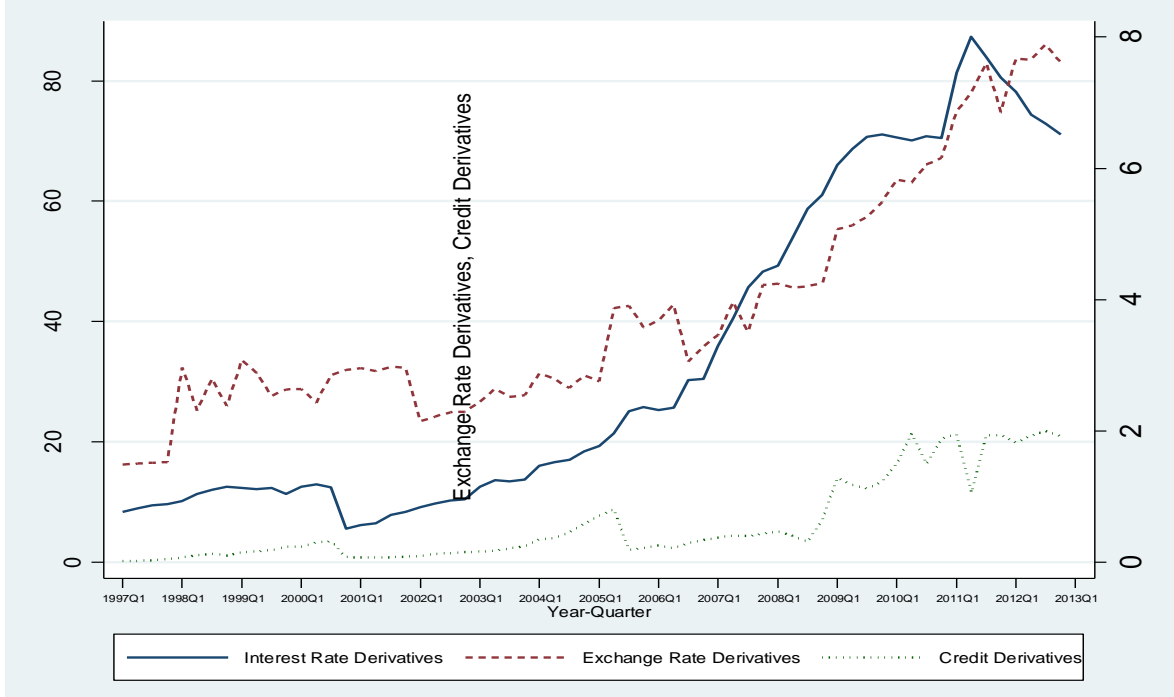

Source: Quarterly data from FR Y-9C, sample period: 1997:Q1-2012:Q4

Our analysis shows that a BHC's use of financial derivatives is associated with its higher exposure towards systematic interest rate risk, exchange rate risk, and credit risk (i.e., nondiversifiable risk exposures that investors cannot trade away on the financial markets). Interestingly, the positive relationship between financial derivatives and systematic risk exposure seems stronger for large BHCs than for small BHCs. These results may indicate that large BHCs with their main operations such as prime brokerage, asset management, proprietary trading, and market making primarily use financial derivatives to derive trading-related gains and that these activities (and the related involvement in derivatives) further expose them to systematic risk. In 
comparison, the results may indicate that small BHCs (with their main operations in deposit taking and commercial lending) employ financial derivatives to a larger extent to hedge against systematic risk.

To further analyze what impact financial derivatives have on systematic risk exposures, we decompose financial derivatives according to their reported purposes. Since March 1995, BHCs are required to report whether their financial derivatives activity is for trading purposes or for purposes other than trading (i.e., for hedging). In Figures 1.3 and 1.4, we report the use of financial derivatives according to their reported purposes in the subsamples of large BHCs and small BHCs.

Figure 1.3. Financial Derivatives Held for Trading Purposes (\$ Trillion)

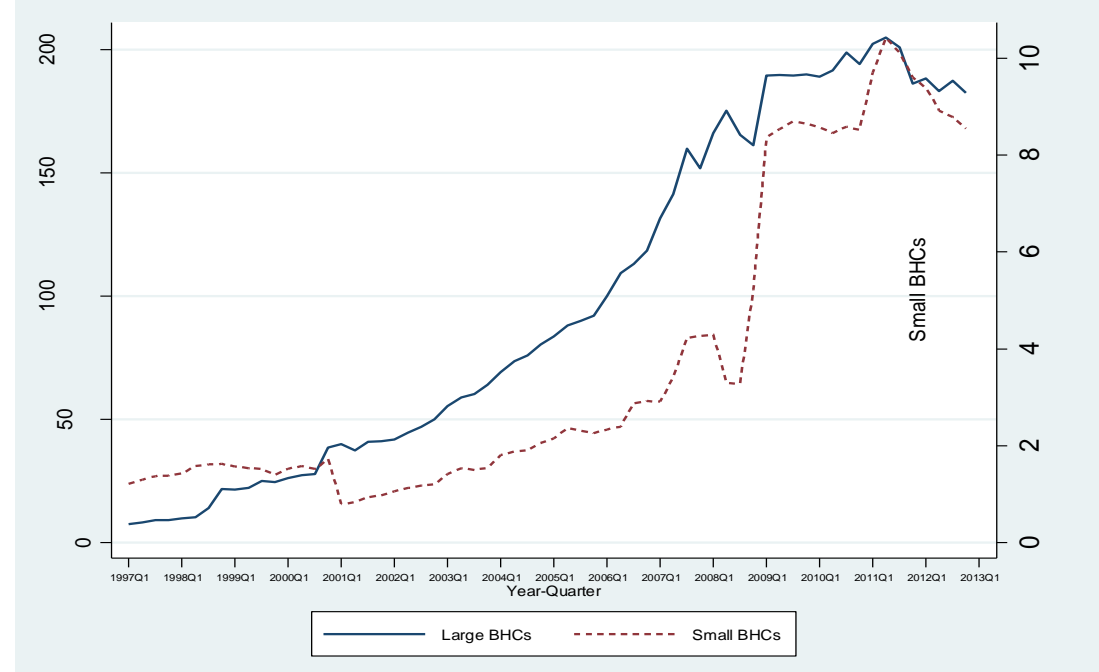

Source: Quarterly data from FR Y-9C, sample period: 1997:Q1-2012:Q4

Figure 1.4. Financial Derivatives Held for Hedging Purposes (\$ Trillion)

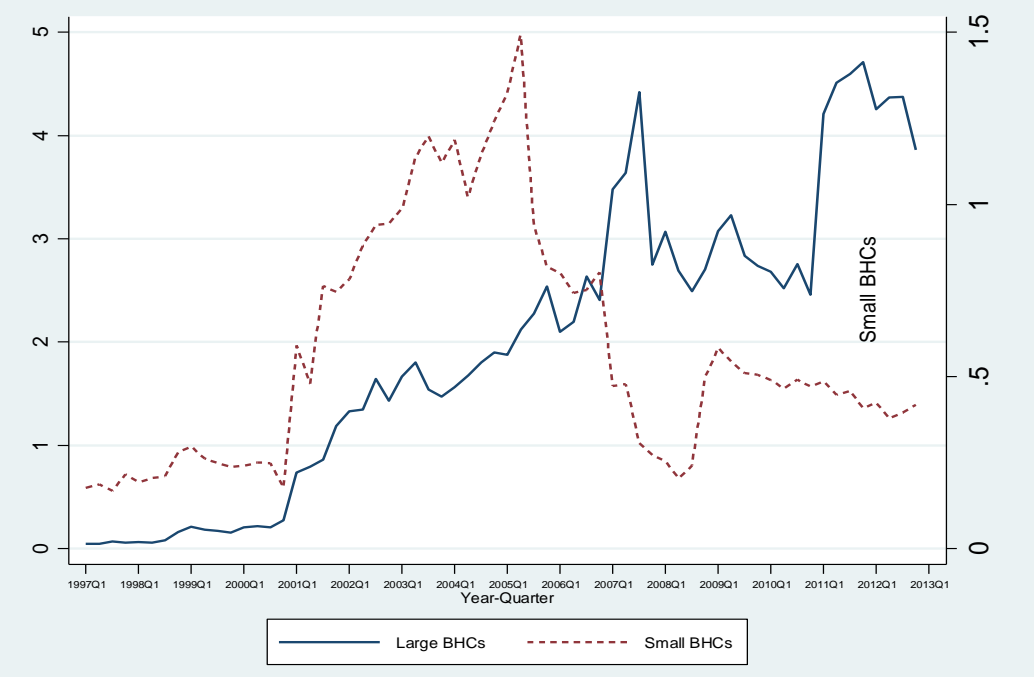

Source: Quarterly data from FR Y-9C, sample period: 1997:Q1-2012:Q4

Our findings show that derivatives held for trading and derivatives held for hedging purposes are both positively and significantly related to BHCs' systematic risk exposures (in the case of interest rate derivatives, exchange rate derivatives, and credit derivatives). This result suggests that the use of financial derivatives might not be aligned with the reported (hedging vs. trading) 
purposes, and that even financial derivatives classified for hedging purposes are associated with higher rather than lower systematic risks.

We also analyze the impact of the global financial crisis on the use of financial derivatives. In the global financial crisis, the relationship between interest rate derivatives and exchange rate derivatives and risk exposures became stronger than in normal times, and the positive relationship between credit derivatives and credit risk became less pronounced.

This chapter is organized as follows; Section 1.3 reviews the literature on financial derivatives and forms hypotheses. Section 1.4 presents the data selection and provides a basic data description. Section 1.5 describes the empirical methodology. Section 1.6 contains the main empirical findings. We analyze how the use of financial derivatives affects BHCs' systematic risk exposures. Section 1.7 concludes this chapter.

\subsection{Relation to the Literature and Hypothesis Formation}

\subsubsection{Relation to the literature}

Empirical literature has been inconclusive about the relationship between financial derivatives use and the risks of BHCs. Choi and Elyasiani (1997) find that the use of financial derivatives further exposes BHCs to risks and that this effect is particularly pronounced for exchange rate risk. Hirtle (1997) finds evidence for the positive relationship between interest rate derivatives and systematic interest rate risk of a BHC (see also Choi, Elyasiani, and Kopecky (1992) and Gunther and Siems (2002)). More recently, Yong, Faff, and Chalmers (2009) were unable to confirm any impact of financial derivatives on exchange rate risk on a sample of Asia-Pacific banks. However, they found that the interest rate derivatives activities are positively associated with long-term interest rate risk exposure but negatively associated with short-term interest rate exposure.

Chaudhry et al. (2000) analyze how different types of exchange rate derivatives affect BHCs' exposure to risks. They find that exchange rate options tend to increase risk, whereas swaps are mainly used to mitigate risk exposures. Carter and Sinkey (1998) focus on large community banks that act as end-users of interest rate derivatives. They find that interest rate derivatives are positively associated with the interest rate risk. Cyree, Huang, and Lindley (2012) show that financial derivatives contributed neither to the increase in bank values in times of growth nor to the depletion of bank values during the global financial crisis. Minton, Stulz, and Williamson (2009) argue that the use of credit derivatives by banks is limited and question the benefits of credit derivatives used for hedging purposes.

Gorton and Rosen (1995) find that banks, especially large dealer banks, use interest rate derivatives mainly to hedge against interest rate risk. Duffee and Zhou (2001) argue that credit derivatives hedge a bank against financial distress and that this additional flexibility allows the bank to avoid the lemon problem due to bank information superiority. In a recent study, Norden, Buston, and Wagner (2011) also find that banks use credit derivatives to improve their credit risk 
management. Mayordomo, Rodriguez-Moreno, and Peña (2014) analyze the impact of financial derivatives on bank systemic risk.

Our main contribution is to disentangle systematic (i.e., undiversifiable) risk into three components - systematic interest rate, exchange rate, and credit risk - at the same time. This allows us to jointly analyze the impact of interest rate derivatives, exchange rate derivatives, and credit derivatives on the corresponding systematic risks. In addition, we analyze how bank-specific characteristics (i.e., the size and the capital of a BHC), the purpose of the financial derivatives (i.e., hedging vs. trading), and the global financial crisis impact the relationship between financial derivatives and systematic risks.

\subsubsection{Hypothesis formation}

Broadly speaking, banks may employ financial derivatives to follow two objectives that sometimes conflict. First, banks may use financial derivatives for hedging purposes to lower their risk exposures. Second, banks may use financial derivatives for speculative purposes to collect revenues and fees (mostly related to financial derivatives trading and origination), which may increase banks' risk exposures.

According to risk management theory (Froot, Scharfstein, and Stein, 1993; Stulz, 1996), banks would employ hedging strategies to lower the costs associated with costly external funding. Raising external funds may be costly for a bank, especially in an unfavorable external environment (see Admati et al. (2012)). A bank partially avoids a refinancing need by hedging uncontrollable risks (Froot, et al., 1993), especially interest rate risk and exchange rate risk, lowering its funding costs. In the spirit of Diamond (1984), banks use financial derivatives to hedge against uncontrollable risks, so that they can focus on their core activity: monitoring their borrowers. Boot and Thakor (1991) argue that banks with large off-balance-sheet activities (e.g., loan commitments) lower their risk exposures compared to banks that lend on a spot market. Their result dwells on the observation that a loan commitment locks a bank into the current interest rate, which mitigates the asset substitution problem of the bank's borrowers if the interest rates rise in the future. If the hedging purpose of financial derivatives prevails, we anticipate that higher derivatives use is associated with lower systematic risk exposures of a BHC.

However, hedging with financial derivatives also has a dark side. Morisson (2005) argues that the informational value of a bank loan ceases to exist if banks can trade on the credit derivatives market. More specifically, when the bank obtains credit default protection, it is no longer exposed to the borrower's potential default. Consequently, the bank can no longer commit to monitoring and screening its borrowers, which increases its risk. ${ }^{8}$

\footnotetext{
${ }^{8}$ In addition, the adverse selection problem may also be present. A bank may want to buy credit protection against the borrowers it perceives as most risky. This is aligned with empirical evidence from Dahiya, Puri, and Saunders (2003) that identifies a significant negative stock price reaction for a borrower when a bank announces that the borrower's loan is to be sold. Dewally and Shao (2012) find that the use of financial derivatives by BHCs increases their opacity.
} 
Large investment banks in particular see their core business in generating and trading financial derivatives and other innovative financial products (Boot and Marinč, 2008). Their engagement in financial derivatives business may then be driven by profit motives rather than by hedging objectives. Banks may use financial derivatives to expose themselves to additional systematic risk exposures and herd with other banks (Acharya and Yorulmazer, 2008). Boot (2014) argues that information technology and related financial innovations, such as the plethora of financial derivatives that originated in the securitization process, have promoted market-driven behavior of banks (see also Boot and Thakor (2010) and Marinč (2013)). Banks have become increasingly exposed to systematic risks on financial markets (and, vice-versa, financial markets are largely affected by banks; see Fiordelisi and Marques-Ibanez (2013)). If this speculative purpose of financial derivatives prevails, we anticipate that financial derivatives will be associated with higher systematic risk exposures of BHCs.

To analyze whether financial derivatives are used for hedging or speculative purposes, we set up the following first hypothesis.

Hypothesis 1.1: Financial derivatives (i.e., interest rate derivatives, exchange rate derivatives, and credit derivatives) impact the (systematic interest rate, exchange rate, and credit) risk of a BHC.

To further analyze the relationship between financial derivatives and risk, we use the classification from Accounting Standards SFAS 133 (Statement of Financial Accounting Standards 133, Accounting for Derivative Instruments and Hedging Activities; FASB, 1998), which demands that banks classify derivatives into two categories: for hedging and for trading purposes. Derivatives are classified for hedging if they are of a certain type (i.e., if they hedge against the changes in the value or cash flows) or if they hedge risks from specific exposures. ${ }^{9}$ In addition, the mitigating relationship between the financial derivative and the hedged risk exposures needs to be proven. This may suggest that financial derivatives for hedging are generally associated with lower risks of a BHC. However, SFAS 133 requires the establishment of the hedging relationship at the level of a specific risk exposure rather than at the enterprise level. It may happen that financial derivatives for hedging may hedge a bank against the specific risk exposure, but increase the risk at the enterprise level. In addition, banks may strive to classify their financial derivative holdings as financial derivatives for hedging rather than for trading due to more favorable regulatory treatment. ${ }^{10}$ Our task is to determine whether derivatives for hedging will lower risks at the level of a BHC.

Hypothesis 1.1a: Financial derivatives for hedging impact risks of a BHC.

\footnotetext{
9 See Ahmed, Kilic, and Lobo (2011) for further description of SFAS 133 and detailed comparison with previous accounting standards SFAS 52 and SFAS 80, including the impact of the accounting change on the hedging effectiveness of derivatives.

${ }^{10}$ Our analysis may relate to the scant literature on regulatory compliance. Bajo et al. (2009) find that family firms and firms with an established corporate ethos comply with regulations more often than others. Considering that family firms are typically small, we anticipate that the smaller size of a BHC might better support regulatory compliance in classification of financial derivatives.
} 
In addition to the hedging purposes, banks also use financial derivatives for trading purposes. Bank derivatives for trading can typically be decomposed into proprietary derivative positions, customer-related derivatives, and positions used for hedging purposes. Whereas proprietary trading in derivatives is considered a profit-generating activity without an expressed hedging objective, the customer-related derivatives and positions used for hedging also have a hedging dimension attached to them. For example, revenues from trading derivatives come from generated fee income and stronger customer relationships. If used for hedging purposes, financial derivatives can prevent financial distress of bank customers that buy them (e.g., small banks and nonfinancial firms), increasing the stability of bank revenues. The direction of the relationship between derivatives for trading and a BHC's risk is therefore ambiguous.

Hypothesis 1.1b: Financial derivatives for trading impact risks of a BHC.

We also aim to analyze the determinants of the relationship between financial derivatives and risks. Undercapitalized banks in particular may use financial derivatives to lower the probability of default and in this way avoid the costs of financial distress (e.g., due to a bank run; see Bauer and Ryser (2004)). In this view, banks would especially hedge the risks that exacerbate the costs of financial distress (see Smith and Stulz (1985) and Stulz (2003)). Consistent with this theory, Purnanandam (2007) shows empirically that banks closer to financial distress hedge against interest rate risk more aggressively.

Rampini and Viswanathan (2010) provide an alternative risk management theory. In their view, financially constrained firms have little leeway in scraping together resources for current investment. ${ }^{11}$ Engaging in costly risk management practices would further exhaust their investment capabilities and more current investment would be foregone. Rampini and Viswanathan (2010) predict that capital-constrained firms hedge less than well-capitalized firms. In our setting, weakly-capitalized BHCs would hedge less than well-capitalized BHCs.

Hypothesis 1.2: The relationship between financial derivatives and risks is affected by a BHC's capital strength.

Another determinant of the relationship between derivatives and risks may be a BHC's size. Bank involvement in dealing and trading in financial derivatives markets requires a substantial investment in capital, skilled employees, and good reputation, which all act as entry barriers for small banks. Tufano (1989) analyzes financial innovations and the first-mover advantage in investment banking in light of the substantial costs associated with the development of a new product. Hunter and Timme (1986) argue that size and technical efficiencies allow large banks to take the lead in financial innovations. Consequently, trading activities of financial derivatives are limited to a set of large banks, whereas smaller banks have little chance to provide full-size risk management services and a broad range of financial derivatives products to their clients.

\footnotetext{
${ }^{11}$ Géczy, Minton, and Schrand (1997) show that corporations use exchange rate derivatives to mitigate cash flow variations, so that they are able to exploit profitable growth opportunities. For further determinants of corporate hedging, see Nance, Smith, and Smithson (1993) and Mian (1996).
} 
Thakor (2012a) builds a theory of financial innovation in which banks offer innovative products that are opaque to investors. Opaqueness of innovations allows for higher profit in a highly competitive banking sector, but at the same time gives rise to refinancing risk, potentially precipitating a financial crisis. Thakor (2012a) predicts a positive relationship between bank opaqueness and financial innovation. Under the assumption that larger banks are also more opaque, the empirical prediction would be that the relationship between financial derivatives and risks is more pronounced for larger banks.

Hypothesis 1.3: The positive relationship between financial derivatives and risks intensifies for larger BHCs.

\subsection{Data Sources, Sample Selection and Data Description}

We combine data from several sources. For financial derivatives data, we collected firm-level data from the FR Y-9C report (Consolidated Financial Statements for Holding Companies) from 1997 to 2012. ${ }^{12}$ We use historical BHCs' stock prices from the Center for Research in Security Prices (CRSP) at the University of Chicago. Macroeconomic data were obtained from the Federal Reserve Board of Governors. ${ }^{13}$ Stock prices and macroeconomic data are monthly data between 1997 and 2012. We also split BHCs into large BHCs (whose total assets are equal to or higher than $\$ 50$ billion) and small BHCs (whose total assets are less than $\$ 50$ billion). ${ }^{14}$ Table 1.1 presents the variables, their definitions, and sources.

Table 1.2 summarizes the use of financial derivatives held by BHCs in the fourth quarter of 2012. Following Guay and Kothari (2003) and Cyree et al. (2012), we use notional principal amounts to depict a BHC's derivatives position. Panel A of Table 1.2 provides a breakdown of financial derivatives by contract types for all U.S. BHCs included in the FR Y-9C report (column 1), the BHCs included in our sample (column 2), and the large BHCs in our sample (column 4). In the fourth quarter of 2012, BHCs' assets reached $\$ 17.6$ trillion and held nearly $\$ 270$ trillion of financial derivative contracts, indicating that the size of financial derivative contracts was more than fifteen times that of the BHCs' total assets. Interest rate derivative contracts (including futures, forwards, swaps, and options) accounted for more than $\$ 228$ trillion and nearly $84 \%$ of the total amount of financial derivatives. Exchange rate derivative contracts and credit derivative contracts accounted for $\$ 31.3$ trillion and $\$ 10.3$ trillion, respectively. Among the interest rate derivative contracts, swaps were the largest individual derivative contract type. They accounted for more than $\$ 155$ trillion and nearly $57 \%$ of interest rate contracts. Among the exchange rate derivative contracts, exchange rate forwards were the most important individual contract type. The value of exchange rate forwards was $\$ 14.3$ trillion, nearly $46 \%$ of all exchange rate derivative contracts. Credit default swaps accounted for $95 \%$ of all credit derivatives held by U.S. BHCs.

\footnotetext{
12 These data are available at: https://www.chicagofed.org/applications/bhc_data/bhcdata_index.cfm.

13 These data are available at: http://www.federalreserve.gov/econresdata/default.htm.

14 Our decomposition is consistent with the classification of sections 165 and 166 of the Dodd-Frank Act, in which BHCs with $\$ 50$ billion or more in consolidated assets are automatically considered to be systemically important institutions (Dodd-Frank Wall Street Reform and Consumer Protection Act and Independent Commission on Banking (2011)). See also http://www.federalreserve.gov/newsevents/testimony/gibson20120516a.htm. 
Table 1.1. Description of Variables

\begin{tabular}{|c|c|c|}
\hline $\begin{array}{l}\text { Variable } \\
\text { First-Stage Variables }\end{array}$ & Definition & Data Sources \\
\hline Stock Return & Measured by the excess rate of return of stock price over the risk-free rate & Center for Research in Security Prices \\
\hline Market Return & Measured by the excess rate of return on market portfolio S\&P 500 over the risk- free rate & Center for Research in Security Prices \\
\hline Interest Rate & Measured by the percentage changes of the price of three-month U.S. treasury bills & $\begin{array}{l}\text { H.15, Federal Reserve Board of Governors } \\
\text { Hons }\end{array}$ \\
\hline Exchange Rate & Measured by the change in the inverse of nominal broad dollar index & $\begin{array}{l}\text { G.5, Federal Reserve Board of Governors } \\
\text { G. }\end{array}$ \\
\hline \multicolumn{3}{|c|}{ Measured by the change of 5 -year BBB bond yield } \\
\hline & (Interest margin) / average interest earning assets & FR Y-9C. BHCK 4074 / earning assets \\
\hline C\&I Loans & Commercial and industrial loans / total assets & FR Y-9C, (BHCK1763 + BHCK1764) / total assets \\
\hline Mortgage Loans & Mortgage loans / total assets & FR Y-9C, (BHCK1410 + BHCK1590) / total assets \\
\hline Other Loans & (Loans-commercial \&industrial loans-mortgage loans) / total assets & FRY-9C, (BHCK2122 - BHCK1766 - BHCK1410 - BHCK1590) / total assets \\
\hline Domestic Deposits & $\begin{array}{l}\text { Domestic deposits / total assets }\end{array}$ & FR Y-9C, $($ BHDM6631 + BHDM6636) / total assets \\
\hline GAP Ratio & $\begin{array}{l}\text { Interest sensitive assets that are repriceable within one year or mature within one year / interest } \\
\text { rate-sensitive liabilities that are repriceable within one vear or mature within one vear }\end{array}$ & FR Y-9C, BHCK3197 / BHCK3296 \\
\hline Interest Rate Exposures & 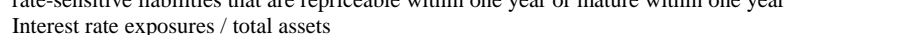 & FR Y-9C, BHCK 8757 / total assets \\
\hline Interest Rate Derivatives for Trading & Notional principal amounts of interest rate contracts for trading purposes / total assets & FR Y-9C, BHCK A126/ total assets \\
\hline Interest Rate Derivatives for Hedging & Notional principal amounts of interest rate contracts for other-than trading purposes / total assets & FR Y-9C, BHCK 8725 / total assets \\
\hline Interest Rate Derivatives & Notional principal amounts of interest rate contracts / total assets & FR Y-9C, (BHCK A126 + BHCK 8725) / total assets \\
\hline Assets in Foreign Currencies & Assets in foreign offices / total assets & FR Y-9C, $($ BHCK0397 + BHCK $1742+$ BHCK $1746+$ BHCK2081 + BHCK 12 \\
\hline Foreign Exchange Deposits & Deposits denominated in foreign currencies and in foreign offices / total assets & FR Y-9C, (BHFN6631 + BHFN6636) / total assets \\
\hline Foreign Exchange Exposures & Foreign exchange exposures / total assets & FR Y-9C, BHCK8758 / total assets \\
\hline Exchange Rate Derivatives for Trading & Notional principal amounts of exchange rate contracts for trading purposes / t & FR Y-9C, BHCK A127 / total assets \\
\hline Exchange Rate Derivatives for Hedging & $\begin{array}{l}\text { Notional principal amounts of exchange rate contracts for other-than trading purposes / total } \\
\text { assets }\end{array}$ & FR Y-9C, BHCK 8726 / total assets \\
\hline Exchange Rate Derivatives & Notional principal amounts of exchange rate contracts / total assets & FR Y-9C, (BHCK A127 + BHCK \\
\hline Market Liquidity & (Cash + securities + fed funds lent) / total assets & FRY-9C, (BHCK0081 + BHCK0395 + BHCK0397 + BHCK1754 + BHCK1773) / total assets \\
\hline Funding Liquidity & (Federal funds sold + securities purchased under agreements) / total assets & FR Y-9C, (BHDMB987 + BHCKB989) / total assets \\
\hline Non-Performing Loans & (Total amount of loans classified as non - performing ) / total assets & FR Y-9C, (BHCK5524 + BHCK5525 + BHCK5526) / total assets \\
\hline Loan Charge-Offs & Loan charge-offs / total assets & FR Y-9C, BHCK4635 / total assets \\
\hline Loan Loss Provisions & Loan loss provisions / total assets & FR Y-9C, BHCK4230 / total assets \\
\hline Credit Exposures & Credit exposures / total assets & FR Y-9C, F186 / total assets \\
\hline Credit Protection Sold & Notional principal amounts of credit risk protection sold / total assets & FR Y-9C, (BHCKC968 + BHCKC970 + BHCKC972 + BHCKC974) / total assets \\
\hline $\begin{array}{l}\text { Credit Protection Bought } \\
\text { Gross Credit Protection }\end{array}$ & $\begin{array}{l}\text { Notional principal amounts of credit risk protection bought / total assets } \\
\text { (Credit risk protection bought + Credit risk protection sold) } / 2\end{array}$ & $\begin{array}{l}\text { FR Y-9C, }(\text { BHCKC } 969+\text { BHCKC971 + BHCKC973 + BHCKC975) / total assets } \\
\text { FRY-9C, ((BHCKC969 + BHCKC971 + BHCKC973 + BHCKC975)/2 + (BHCKC968 + BHCKC } 970+\text { BHCKC972 + }\end{array}$ \\
\hline Net Credit Protection Bought & (Credit risk protection bought - Credit risk protection sold) & $\begin{array}{l}\text { BHCKC974) / 2) / total assets } \\
\text { FR Y-9C, ((BHCKC969 + BHCKC971 + BHCKC973 + BHCKC975) - (BHCKC968 + BHCKC970 + BHCKC972 + } \\
\text { BHCKC974) / total assets }\end{array}$ \\
\hline Credit Derivatives & (Credit risk protection bought + Credit risk protection sold) & 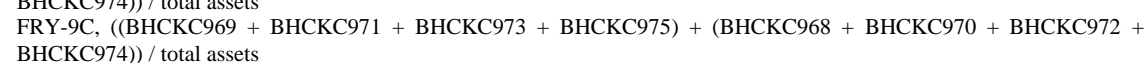 \\
\hline Size & $\log$ (total assets) & FR Y-9C, $\log ($ BHCK2170) \\
\hline Capital Ratio & Total risk-based capital ratio & FR Y-9C, BHCK7205 \\
\hline Tier 1 Ratio & Tier 1 capital ratio & FRY-9C, BHCK 8274 / BHCK2170 \\
\hline & The GDP growth in each state & http://www.bea.gov/regional/ \\
\hline $\begin{array}{l}\text { GDP Grown } \\
\text { Income Tax Rate }\end{array}$ & $\begin{array}{l}\text { Corporate income tax rates in each state, as the data before } 2000 \text { is missing, we use the income } \\
\text { tax rate in } 2000 \text { to measure the corporate income tax rates from 1997-1999 }\end{array}$ & www.taxfoundation.org \\
\hline Crisis & $\begin{array}{l}\text { Equals to } 1 \text { during the financial crisis (i.e., from the third quarter of } 2007 \text { to the fourth quarter of } \\
2010 \text { and } 0 \text { otherwise. }\end{array}$ & \\
\hline SIFI & Equals to 1 if the asset of the BHCs is larger or equal to $\$ 50$ billion, and 0 otherwise. & \\
\hline Total Financial Derivative & Interest Rate Derivatives + Exchange Rate Derivatives+ Credit Derivatives & \\
\hline Financial Derivatives for Trading & $\begin{array}{l}\text { Interest Rate Derivatives for Trading + Exchange Rate Derivatives for Trading + Gross Credit } \\
\text { Protection }\end{array}$ & \\
\hline Financial Derivatives for Hedging & $\begin{array}{l}\text { Inteection } \\
\text { Protest Rate Derivatives Bought }\end{array}$ & \\
\hline
\end{tabular}


Column 2 of Panel A summarizes financial derivatives data in the BHCs in our sample and compares them to the total U.S. BHCs (column 3). The total assets of the BHCs in our sample were almost $\$ 11.9$ trillion, which accounted for $68 \%$ of the total assets of total reported U.S. BHCs. The financial derivative contracts used by the BHCs in our sample were more than \$221 trillion; that is, $82 \%$ of the total amount. This indicates that our sample BHCs are a good reflection of U.S. BHCs and the U.S. financial derivatives market.

Column 4 of Panel A summarizes financial derivatives by the large BHCs in our sample and compares this to the total U.S. BHCs (column 5) and to the BHCs in our sample (column 6). Large BHCs held $\$ 11.2$ trillion of total assets, accounting for $64 \%$ of the total assets of all U.S. BHCs and $94 \%$ of total assets of the BHCs in our sample. Large BHCs held more than $\$ 182$ trillion of financial derivative contracts, which accounts for $68 \%$ of the total BHCs' financial derivative contracts and $82 \%$ of the financial derivative contracts in our sample. This shows that large BHCs are the main participants in the U.S. financial derivatives market.

Panel B of Table 1.2 presents the breakdown of financial derivatives with respect to their reported purposes (trading vs. hedging). More than $\$ 253$ trillion (out of $\$ 270$ trillion held by BHCs in total) of financial derivative contracts were held for trading, which accounts for over $94 \%$ of all financial derivative contracts. The financial derivatives held for trading were mainly (i.e., $71 \%$ of them) concentrated in large BHCs. Concentration of financial derivatives in large BHCs was less pronounced for financial derivatives held for hedging purposes. Large BHCs held $65.6 \%$ of all financial derivatives for hedging purposes. The implication is that the small BHCs in our sample predominantly act as end-users in the financial derivatives market and mainly classify financial derivatives for hedging purposes.

Table 1.2. Financial Derivatives Used by U.S. BHCs (Notional Principal Amounts, \$ billion)

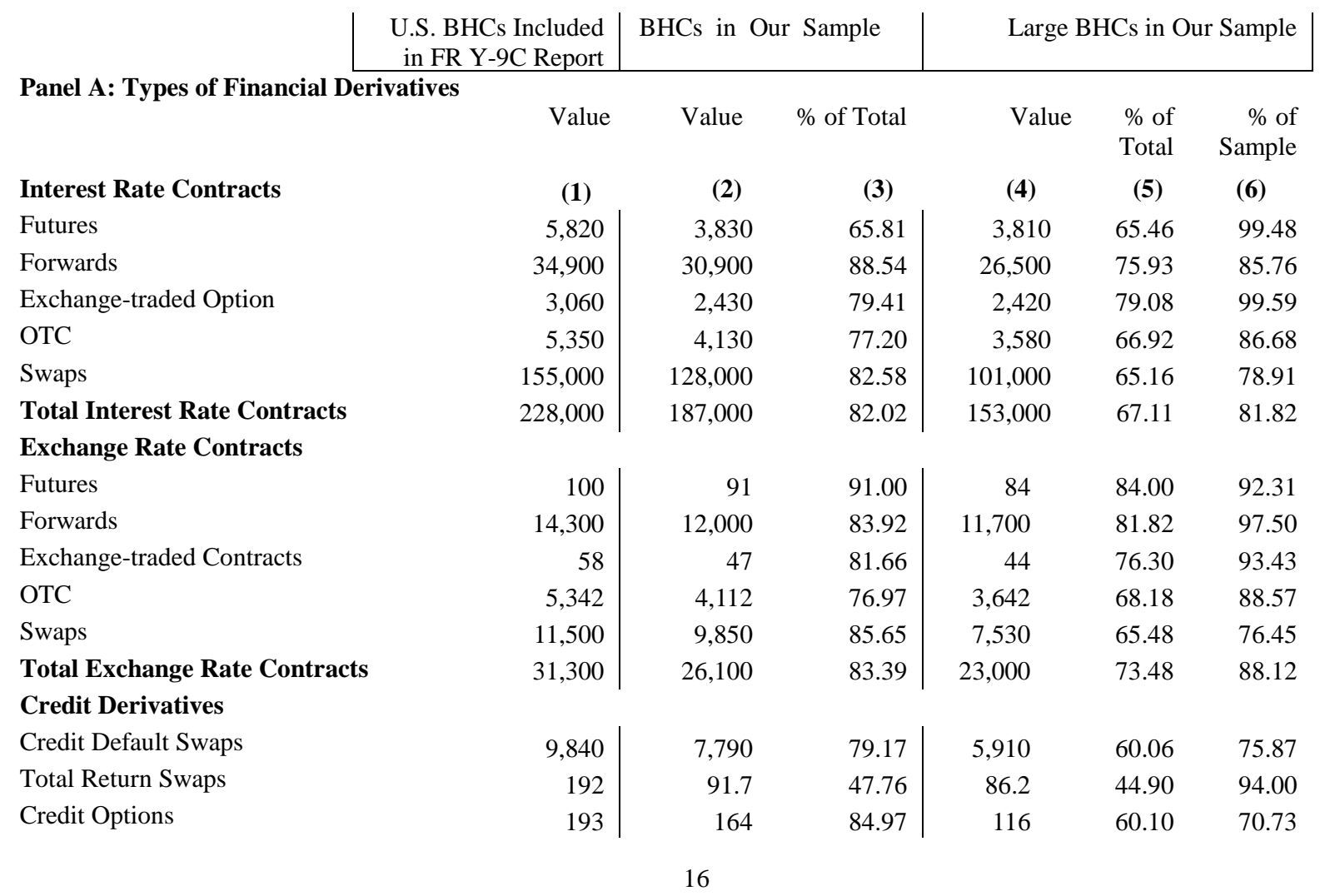


Table 1.2. Financial Derivatives Used by U.S. BHCs (Notional Principal Amounts, \$ billion)

\begin{tabular}{|c|c|c|c|c|c|c|}
\hline \multirow[b]{2}{*}{ Other Credit Derivatives } & $\begin{array}{l}\text { U.S. BHCs Included } \\
\text { in FR Y-9C Report }\end{array}$ & \multicolumn{2}{|c|}{ BHCs in Our Sample } & \multicolumn{3}{|c|}{ Large BHCs in Our Sample } \\
\hline & 105 & 98.6 & 96.19 & 101 & 93.90 & 97.62 \\
\hline Total Credit Derivatives & 10,330 & 8,144 & 78.84 & 6,213 & 60.15 & 76.29 \\
\hline Total Assets & 17,600 & 11,900 & 67.61 & 11,200 & 63.64 & 94.12 \\
\hline Total Financial Derivatives & 269,630 & 221,244 & 82.05 & 182,213 & 67.58 & 82.36 \\
\hline \multicolumn{7}{|c|}{ Panel B: Financial Derivatives for Different Purposes } \\
\hline $\begin{array}{l}\text { Interest Rate Contracts Held For } \\
\text { Trading }\end{array}$ & 223,000 & 184,000 & 82.51 & 149,000 & 66.82 & 80.98 \\
\hline $\begin{array}{l}\text { Interest Rate Contracts Held for } \\
\text { Hedging }\end{array}$ & 4,110 & 3,270 & 79.56 & 3,250 & 79.08 & 99,29 \\
\hline $\begin{array}{l}\text { Foreign Exchange Contracts Held For } \\
\text { Trading }\end{array}$ & 30,400 & 25,500 & 83.88 & 22,400 & 73.68 & 87.84 \\
\hline $\begin{array}{l}\text { Foreign Exchange Contracts Held for } \\
\text { Hedging }\end{array}$ & 861 & 609 & 70.73 & 599 & 69.57 & 98.37 \\
\hline Credit Protection Sold & 9,910 & 7,900 & 79.72 & 6,000 & 60.54 & 75.59 \\
\hline Credit Protection Bought & 10,100 & 7,940 & 78.61 & 6,040 & 59.80 & 76.07 \\
\hline
\end{tabular}

Note. The financial data are for the 4th quarter of 2012 from FR Y-9C.

Table 1.3 compares the means of on-balance-sheet and off-balance-sheet variables for the total sample BHCs and the two subsamples (i.e., large BHCs and small BHCs) from 1997 to 2012. We report the means, the difference in means, and $t$ statistics based on unequal group variance. Panel A depicts the use of interest rate derivatives. Large BHCs have a lower interest margin ratio, more loans and deposit, and more interest rate derivatives than small BHCs. Panel B focuses on exchange rate variables. Large BHCs have more foreign currency assets and deposits, and are more active in the exchange rate derivatives market than small BHCs. Panel $\mathrm{C}$ depicts credit risk variables. Large BHCs have higher market liquidity and funding liquidity, more loan charge-offs, and more loan provisions and non-performing loans, and they hold more credit derivatives than small BHCs. Panel D presents control variables. Large BHCs' total assets are thirty times higher than those of small BHCs, they have a lower capital ratio, and they hold more financial derivatives.

Table 1.3 indicates that large BHCs mainly focus on trading-related activities in the financial derivatives business, whereas small BHCs use financial derivatives products mainly for hedging purposes as end-users.

Table 1.3. Difference in Means: Financial Characteristics of BHCs, Large BHCs, and Small BHCs

\begin{tabular}{|c|c|c|c|c|c|c|}
\hline \multirow[b]{2}{*}{ Variable } & \multicolumn{2}{|c|}{ Group Means } & \multirow[b]{2}{*}{$\begin{array}{c}\text { Small (S) } \\
\text { BHCs }\end{array}$} & \multicolumn{3}{|c|}{ Difference in Means } \\
\hline & Total Sample & $\begin{array}{c}\text { Large }(\mathrm{L}) \\
\text { BHCs }\end{array}$ & & $\mathrm{L}-\mathrm{S}$ & $t$-statistic & $p$-value \\
\hline \multicolumn{7}{|l|}{ Panel A: Interest Rate Variables } \\
\hline Interest Margin Ratio & 0.021 & 0.019 & 0.022 & -0.003 & $-9.543^{* * *}$ & 0.0000 \\
\hline C\&I Loans & 0.108 & 0.143 & 0.106 & 0.037 & $15.623^{* * *}$ & 0.0000 \\
\hline Mortgage Loans & 0.473 & 0.301 & 0.484 & -0.183 & $-48.421^{* * *}$ & 0.0000 \\
\hline Other Loans & 0.074 & 0.147 & 0.069 & 0.078 & $32.690^{* * *}$ & 0.0000 \\
\hline Domestic Deposits & 0.734 & 0.548 & 0.746 & -0.198 & $-36.013^{* * *}$ & 0.0000 \\
\hline GAP Ratio & 0.051 & 0.302 & 0.051 & 0.269 & $3.135^{* * *}$ & 0.0021 \\
\hline Interest Rate Options Bought & 0.040 & 0.250 & 0.026 & 0.223 & $13.217^{* * *}$ & 0.0000 \\
\hline Interest Rate Options Written & 0.035 & 0.249 & 0.021 & 0.229 & $13.024^{* * *}$ & 0.0000 \\
\hline Interest Rate Forwards \& Futures & 0.058 & 0.443 & 0.031 & 0.412 & $14.009^{* * *}$ & 0.0000 \\
\hline Interest Rate Swaps & 0.219 & 1.956 & 0.101 & 1.855 & $13.187^{* * *}$ & 0.0000 \\
\hline Interest Rate Derivatives for Trading & 0.303 & 2.699 & 0.140 & 2.559 & $12.901^{* * *}$ & 0.0000 \\
\hline
\end{tabular}


Table 1.3. Difference in Means: Financial Characteristics of BHCs, Large BHCs, and Small BHCs

\begin{tabular}{|c|c|c|c|c|c|c|}
\hline \multirow[b]{2}{*}{ Variable } & \multicolumn{2}{|c|}{ Group Means } & \multirow[b]{2}{*}{$\begin{array}{c}\text { Small (S) } \\
\text { BHCs }\end{array}$} & \multicolumn{3}{|c|}{ Difference in Means } \\
\hline & Total Sample & $\begin{array}{c}\text { Large }(\mathrm{L}) \\
\text { BHCs }\end{array}$ & & $\mathrm{L}-\mathrm{S}$ & $t$-statistic & $p$-value \\
\hline Interest Rate Derivatives for Hedging & 0.039 & 0.158 & 0.031 & 0.127 & $20.847^{* * *}$ & 0.0000 \\
\hline Interest Rate Derivatives & 0.352 & 2.902 & 0.179 & 2.724 & $13.647^{* * *}$ & 0.0000 \\
\hline \multicolumn{7}{|l|}{ Panel B: Exchange Rate Variables } \\
\hline Assets in Foreign Currencies & 0.004 & 0.033 & 0.002 & 0.031 & $15.866^{* * *}$ & 0.0000 \\
\hline Foreign Currency Deposits & 0.010 & 0.070 & 0.005 & 0.065 & $21.162^{* * *}$ & 0.0000 \\
\hline Exchange Rate Options Bought & 0.005 & 0.036 & 0.003 & 0.033 & $13.005^{* * *}$ & 0.0000 \\
\hline Exchange Rate Options Written & 0.006 & 0.036 & 0.003 & 0.033 & $12.465^{* * *}$ & 0.0000 \\
\hline Exchange Rate Forwards \& Futures & 0.043 & 0.410 & 0.017 & 0.393 & $16.435^{* * *}$ & 0.0000 \\
\hline Exchange Rate Swaps & 0.009 & 0.077 & 0.005 & 0.072 & $12.554^{* * *}$ & 0.0000 \\
\hline Spot Exchange Rate & 0.005 & 0.037 & 0.002 & 0.035 & $16.087^{* * *}$ & 0.0000 \\
\hline Exchange Rate Derivatives for Trading & 0.061 & 0.545 & 0.028 & 0.517 & $17.055^{* * *}$ & 0.0000 \\
\hline Exchange Rate Derivatives for Hedging & 0.002 & 0.012 & 0.001 & 0.011 & $16.416^{* * *}$ & 0.0000 \\
\hline Exchange Rate Derivatives & 0.063 & 0.560 & 0.029 & 0.531 & $17.346^{* * *}$ & 0.0000 \\
\hline \multicolumn{7}{|l|}{ Panel C: Credit Risk Variables } \\
\hline Market Liquidity & 0.263 & 0.246 & 0.264 & -0.018 & $-5.095^{* * *}$ & 0.0000 \\
\hline Funding Liquidity & 0.014 & 0.027 & 0.013 & 0.014 & $9.4566^{* * *}$ & 0.0000 \\
\hline Non-Performing Loans & 0.019 & 0.017 & 0.019 & -0.002 & $-3.175^{* * *}$ & 0.0004 \\
\hline Loan Charge-Offs & 0.003 & 0.004 & 0.003 & 0.001 & $9.497^{* * *}$ & 0.0000 \\
\hline Loan Loss Provisions & 0.003 & 0.004 & 0.003 & 0.001 & $4.993^{* * *}$ & 0.0000 \\
\hline Total Return Swaps & 0.0005 & 0.0033 & 0.0002 & 0.0003 & $9.497^{* * *}$ & 0.0000 \\
\hline Credit Option & 0.0002 & 0.0011 & 0.0002 & 0.0010 & $5.593^{* * *}$ & 0.0000 \\
\hline Other Credit Derivatives & 0.0002 & 0.0022 & 0.0001 & 0.0021 & $10.268^{* * *}$ & 0.0000 \\
\hline Credit Protection Sold & 0.009 & 0.071 & 0.005 & 0.066 & $8.999^{* * *}$ & 0.0000 \\
\hline Credit Protection Bought & 0.010 & 0.076 & 0.004 & 0.072 & $9.376^{* * *}$ & 0.0000 \\
\hline Credit Derivatives & 0.019 & 0.147 & 0.010 & 0.137 & $9.210^{* * *}$ & 0.0000 \\
\hline \multicolumn{7}{|l|}{ Panel D: Control Variables } \\
\hline Total Assets ( $\$$ billion) & 25.3 & 275 & 8.54 & 253.59 & $20.804^{* * *}$ & 0.0000 \\
\hline Capital Ratio (\%) & 14.08 & 12.77 & 14.17 & -1.40 & $-9.568^{* * *}$ & 0.0000 \\
\hline GDP Growth (\%) & 1.79 & 1.81 & 1.78 & 0.031 & 0.395 & 0.6927 \\
\hline Tier 1 ratio $(\%)$ & 8.684 & 7.412 & 8.771 & -1.360 & $-22.231^{*}$ & 0.0000 \\
\hline Income Tax Rate (\%) & 41.90 & 42.11 & 41.88 & 0.23 & $3.435^{* * *}$ & 0.0001 \\
\hline
\end{tabular}

Note. $* p<0.10 * * p<0.05, * * * p<0.01$. The $t$-statistics are based on unequal group variances. Variables used are described in Table 1.1.

Source: The financial data are between 1997 and 2012 and from Financial Statement of FR Y-9C.

\subsection{Empirical Methodology}

The empirical analysis employs a two-stage time-series cross-section regression model to examine the relationship between systematic risk and the use of financial derivatives. The regression proceeds in two stages (consistent with Fama and French (1992)). In the first stage, the stock return of each BHC is regressed against the changes in the market return, interest rate, exchange rate, and credit spread. In this way, we obtain risk betas that measure the BHC's systematic (i.e., nondiversifiable) risk exposure towards market risk, interest rate risk, exchange rate risk, and credit risk. In the second-stage regression, the risk betas are regressed against the on-balance-sheet variables and financial derivatives variables.

First-Stage Regression: The monthly stock returns of publicly traded BHCs are used to measure systematic exposures of each bank towards market risk, interest rate risk, exchange rate risk, and credit risk. Such a multi-factor model has also been employed by Flannery and James (1984), Choi and Elyasiani (1997), and Hirtle (1997). The first-stage regression is as follows: 
Stock Return $_{\mathrm{it}}=\alpha_{\mathrm{i}}+\beta_{\text {Market }, i t}$ Market Return $_{\mathrm{it}}+\beta_{\text {Interest }, \text { It }}$ Interest Rate $_{\mathrm{it}}$

$$
+\beta_{\text {Exchange }, \text { Et }} \text { Exchange Rate }{ }_{\mathrm{it}}+\beta_{\text {Credit }, \text { Cr }} \text { Credit Risk }_{\mathrm{it}}+\varepsilon_{\mathrm{it}}
$$

where $\beta_{\text {Market, } i t}, \beta_{\text {Interest }, i t}, \beta_{\text {Exchange }, i t}, \beta_{\text {Credit }, i t}$ are systematic risk exposures of BHC $i$ towards market risk, interest rate risk, exchange rate risk, and credit risk at time $t$, respectively; $\alpha_{i}$ are constant error terms, and $\varepsilon_{i t}$ are random error terms.

The dependent variable Stock Return is the excess rate of stock return over the risk-free rate (i.e., annualized rate on three-month U.S. treasury bills). The independent variable Market Return denotes the excess rate of return on the Standard and Poor's 500 index over the risk-free rate; Interest Rate is defined as the rate of change in the price of the three-month U.S. treasury bill rate (i.e., $\left.\left(\sqrt[4]{\frac{1+r_{t-1}}{1+r_{t}}}-1\right)\right)$, where $r$ is the annualized rate on three-month U.S. treasury bills; following Francis, Hasan, and Hunter (2008), Exchange Rate is the rate of change in the inverse of the nominal broad dollar index (i.e., $\left.\left(\mathrm{e}_{t}-\mathrm{e}_{t-1}\right) / \mathrm{e}_{t-1}\right)$, where $e_{t}$ is the value of basket of foreign currencies against the U.S. dollar at time $t,{ }^{15}$ and Credit Risk is defined as the change of BBB bond yield (i.e., $\left.\left(\mathrm{b}_{t}-\mathrm{b}_{t-1}\right) / \mathrm{b}_{t-1}\right)$, where $b_{t}$ is the five-year corporate BBB bond yield in the U.S. market at time $t$. All data are calculated on a monthly basis.

To adjust for possible bias due to cross-equation dependencies, the regression equations for each BHC are estimated as a simultaneous equation system, using a modified seemingly unrelated technique (SUR). The modified SUR technique, developed by Chamberlain (1982), MaCurdy (1982), and Choi and Elyasiani (1997), is a variation of the standard SUR method and produces asymptotically efficient estimates without imposing either conditional homoskedasticity or serial independence restrictions on disturbance terms. ${ }^{16}$

The market model regressions are performed quarterly by using a four-year rolling window between 1997 and 2012 to estimate quarterly-varying beta coefficients for each BHC. This process results in separate risk betas for each BHC for each quarter in the sample. ${ }^{17}$ The values of $\beta_{\text {Market }, i t}, \beta_{\text {Interest }, i t}, \beta_{\text {Exchange }, i t}$, and $\beta_{\text {Credit }, i t}$ are therefore quarterly and bank-specific data, and are treated as panel data in the second-stage regression.

Second-Stage Regression: In the second stage, we follow Hutson and Stevenson (2009), Choi and Jiang (2009), and Bredin and Hyde (2011), and regress betas (i.e., interest rate risk $\beta_{\text {Interest,it, }}$ exchange rate risk $\beta_{\text {Exchange,it, }}$ and credit risk $\beta_{\text {Credit,it }}$ ) generated in the first stage in a panel data

\footnotetext{
15 The nominal broad dollar index is a weighted average of the foreign exchange value of the U.S. dollar against the currencies of a broad group of major U.S. trading partners. Weights for the broad index can be found at http://www.federalreserve.gov/releases/H10/Weights. For more information on exchange rate indexes for the U.S. dollar, see "Indexes of the Foreign Exchange Value of the Dollar," Federal Reserve Bulletin, 91:1 (Winter 2005), pp. 1-8 (http://www.federalreserve.gov/pubs/bulletin/2005/winter05_index.pdf).

${ }_{16}$ The SUR regression has been employed in recent studies by Viale, Kolari, and Fraser (2009), Yong et al. (2009), Ammer, Vega, and Wongswan (2010), Białkowski, Etebari, and Wisniewski (2012), and Lim, Sum, and Khun (2012).

17 A number of BHCs drop out of the sample because of mergers and failures during our sample period.
} 
regression against bank-specific on-balance-sheet and off-balance-sheet (i.e., financial derivatives) variables. To increase the accuracy of second-stage estimation, we follow Doidge, Griffin, and Williamson (2006) and Chue and Cook (2008), and weight each observation by the inverse of the standard errors of $\beta_{\text {Interest,it, }} \beta_{\text {Exchange, it, }}$ and $\beta_{\text {Credit,it }}$, obtained in the first stage. With this procedure, the betas that are estimated more precisely in the first-stage regression receive a heavier weight in the second-stage regression.

The equations can be written as follows:

$\beta_{\text {Interest }, i t}=\gamma_{i}+\sum_{j} \delta_{j} \mathrm{X}_{j i t}+\sum_{\mathrm{j}}\left(\eta_{j}+\mu_{\mathrm{j}} \mathrm{Z}_{j i t}\right) \mathrm{Y}_{j i t}+\sum_{j} \varrho_{j} Z_{j i t}+\mathrm{v} \mathrm{YEAR}_{t}+\epsilon_{i t}$

where $X_{j i t}$ are on-balance-sheet variables (including Interest Margin, C\&I Loans, Mortgage Loans, Other Loans, and Domestic Deposits) and three control variables (Size, Capital Ratio, and GDP Growth), and $Y_{j i t}$ are the notional principal amounts of interest rate derivatives used. In a slightly changed specification, $Y_{j i t}$ can be Interest Rate Derivatives for Trading and Interest Rate Derivatives for Hedging. Potential determinants of the relationship between financial derivatives and risks are included in $Z_{j i t}$ and include the crisis dummy variable $C r i s i s_{t}$, the level of regulatory capital ratio Capital Ratio $_{i t}$ and Tier 1 Ratio $_{i t}$, size variables Size $i t$, and the dummy variable SIFI, denoting a large BHC (see Table 1.1 for precise definition of variables). The regression also includes the bank-specific fixed effects and yearly dummy variables to control for macroeconomic factors that may vary over time. Standard errors $\left(\varepsilon_{i t}\right)$ are heteroskedasticity-consistent.

$\beta_{\text {Exchange }, i t}=\Phi_{i}+\sum_{j} \xi_{j} \mathrm{~A}_{j i t}+\sum_{\mathrm{j}}\left(\varsigma_{j}+\omega_{\mathrm{j}} \mathrm{Z}_{j i t}\right) \mathrm{B}_{j i t}+\sum_{j} \varphi_{j} Z_{j i t}+\rho \mathrm{YEAR}_{t}+\epsilon_{i t}$

where $A_{j i t}$ are on-balance-sheet variables (including Assets in Foreign Currencies and Foreign Exchange Deposits) and three control variables (Size, Capital Ratio, and GDP Growth) and $B_{j i t}$ are the notional principal amounts of exchange rate derivatives used. In a slightly changed specification, $B_{j i t}$ can be Exchange Rate Derivatives for Trading and Exchange Rate Derivatives for Hedging.

$\beta_{\text {Credit }, i t}=\Psi_{i}+\sum_{j} \mu_{j} \mathrm{O}_{j i t}+\sum_{\mathrm{j}}\left(v_{j}+\tau_{\mathrm{j}} \mathrm{Z}_{j i t}\right) \mathrm{P}_{j i t}+\sum_{j} \kappa_{j} Z_{j i t}+\sigma \mathrm{YEAR}_{t}+\epsilon_{i t}$

where $O_{j i t}$ are on-balance-sheet variables (including Market Liquidity, Funding Liquidity, Non-Performing Loans, Loan Charge-Offs, and Loan Loss Provisions) and three control variables (Size, Capital Ratio, and GDP Growth), and $P_{j i t}$ are the notional principal amounts of credit derivatives used. In a slightly changed specification, $P_{j i t}$ can be Gross Credit Protection and Net Credit Protection Bought. 


\subsection{Empirical Results}

\subsubsection{First-stage regression results}

In the first stage, we estimate the beta coefficients of market risk, interest rate risk, exchange rate risk, and credit risk for each $\mathrm{BHC}$ in each quarter in our sample.

Correlations between the first-stage variables are shown in Table 1.4. We observe from Table 1.4 that excess stock returns are significantly negatively correlated with the changes in bond yields, but significantly positively correlated with changes in interest rate and exchange rate. Excess stock returns are positively and significantly correlated with excess market returns for the total sample and small BHCs, whereas the relationship is insignificant for large BHCs.

\section{Table 1.4. Correlation Coefficients Between Macroeconomic Factors}

This table indicates the extent of multicollinearity, if any, between the various variables used to determine the interest rate, exchange rate, and credit sensitivities for all bank holding companies(BHCs), the top group BHCs, the median group BHCs, and the bottom BHCs in Panel A, B, C, and D, respectively. The variables are the excess stock returns (SR), the excess market return (MKT), the changes on the price of three-month US Treasury bills (IR), the change in the inverse of nominal broad dollar index (FX), and the change in the BBB bond yield (CREDIT).

\begin{tabular}{|c|c|c|c|c|c|}
\hline \multicolumn{6}{|c|}{ Panel A: Total Sample } \\
\hline & SR & MKT & IR & FX & CREDIT \\
\hline SR & 1 & & & & \\
\hline MKT & $0.395 * * *$ & 1 & & & \\
\hline IR & $0.0424 * * *$ & $-0.178 * * *$ & 1 & & \\
\hline FX & $0.232 * * *$ & $0.526^{* * *}$ & $-0.0633 * * *$ & 1 & \\
\hline CREDIT & $-0.0783 * * *$ & $-0.284 * * *$ & 0.00590 & $-0.432 * * *$ & 1 \\
\hline \multicolumn{6}{|c|}{ Panel B: Large Group } \\
\hline & SR & MKT & IR & FX & CREDIT \\
\hline SR & 1 & & & & \\
\hline MKT & $0.515 * * *$ & 1 & & & \\
\hline IR & -0.00359 & $-0.176^{* * *}$ & 1 & & \\
\hline $\mathrm{FX}$ & $0.281 * * *$ & $0.517 * * *$ & $-0.0617 * * *$ & 1 & \\
\hline CREDIT & $-0.127 * * *$ & $-0.281 * * *$ & 0.00226 & $-0.428 * * *$ & 1 \\
\hline \multicolumn{6}{|c|}{ Panel C: Small Group } \\
\hline & SR & MKT & IR & FX & CREDIT \\
\hline SR & 1 & & & & \\
\hline MKT & $0.348 * * *$ & 1 & & & \\
\hline IR & $0.0607 * * *$ & $-0.179 * * *$ & 1 & & \\
\hline FX & $0.213 * * *$ & $0.529 * * *$ & $-0.0639 * * *$ & 1 & \\
\hline CREDIT & $-0.0593 * * *$ & $-0.286 * * *$ & 0.00733 & $-0.433 * * *$ & 1 \\
\hline
\end{tabular}

In Table 1.5, we report the results of the multifactor index model based on the entire sample period for the total sample, large BHCs, and small BHCs. The results indicate that market risk beta $\left(\beta_{\text {Market }}\right)$ and interest rate beta $\left(\beta_{\text {Interest }}\right)$ are statistically significant (at the $1 \%$ level on two-tailed tests) for the total sample and for two subsamples (large BHCs and small BHCs). Exchange rate beta ( $\left.\beta_{\text {Exchange}}\right)$ is significant at the $1 \%$ level for the total sample and small BHCs, but for the large BHCs at a lower level $(10 \%)$. Credit risk beta $\left(\beta_{\text {Credit }}\right)$ is significant in all cases (at least at the $5 \%$ level).

Table 1.5 compares risk betas across large and small BHCs. Market risk beta $\left(\beta_{\text {Market }}\right)$ is higher 
for large BHCs, followed by the total sample and small BHCs. This is consistent with the popular notion that large BHCs, acting as market makers and holding a large proportion of financial derivatives for trading purposes, are more exposed to market risk (Standard and Poor's, 2011). In contrast, small BHCs are more sensitive to systematic interest rate risk, exchange rate risk, and credit risk than large BHCs. Higher sensitivity to systematic interest rate risk and credit risk is aligned with the notion that lending (and associated credit risk) is the core business of small BHCs.

Table 1.5. First-Stage Estimation of Risks Betas

\begin{tabular}{|c|c|c|c|c|c|}
\hline \multicolumn{6}{|c|}{ Panel A: Regression Results } \\
\hline \multirow{3}{*}{ Total Sample BHCs } & Intercept & $\beta_{\text {Market }}$ & $\beta_{\text {Interest }}$ & $\beta_{\text {Exchange }}$ & $\beta_{\text {Credit }}$ \\
\hline & $0.00352 * * *$ & $0.893 * * *$ & $0.654 * * *$ & $0.340 * * *$ & $0.132 * * *$ \\
\hline & $(4.42)$ & $(44.32)$ & $(15.10)$ & $(5.35)$ & (6.89) \\
\hline \multirow[t]{2}{*}{ Large BHCs (L) } & $0.00328 * *$ & $1.155 * * *$ & $0.504 * * *$ & $0.203^{*}$ & $0.0759 * *$ \\
\hline & $(2.32)$ & $(32.55)$ & $(6.58)$ & $(1.81)$ & $(2.23)$ \\
\hline \multirow[t]{2}{*}{ Small BHCs (S) } & $0.00362 * * *$ & $0.788 * * *$ & $0.713 * * *$ & $0.400 * * *$ & $0.155^{* * *}$ \\
\hline & $(3.79)$ & $(32.51)$ & $(13.69)$ & $(5.25)$ & $(6.70)$ \\
\hline Group Difference (L - S) & -0.00034 & 0.367 & -0.209 & -0.197 & -0.0791 \\
\hline$[p$-value: $\mathrm{L}=\mathrm{S}]$ & {$[0.299]$} & {$[0.000 * * *]$} & {$[0.000 * * *]$} & {$[0.049 * *]$} & {$[0.000 * * *]$} \\
\hline Panel B:Regression & Total Sample & Large & \multirow{2}{*}{\multicolumn{2}{|c|}{ Small BHCs }} & \\
\hline Statistics & $\mathrm{BHCs}$ & $\mathrm{BHCs}$ & & & \\
\hline Adjusted-R2 & 0.173 & 0.273 & \multicolumn{2}{|c|}{0.142} & \\
\hline$N$ & 14102 & 3982 & \multicolumn{2}{|c|}{10120} & \\
\hline
\end{tabular}

In Table 1.6, we focus on a subset of BHCs that started using the financial derivatives (interest rate derivatives, exchange rate derivatives, or credit derivatives) during the sample period and compare their risk exposures before and after the use of the financial derivatives. The BHCs are exposed to a higher level of systematic interest rate risk, exchange rate risk, credit risk, and market risk if they start using interest rate derivatives, exchange rate derivatives, and credit derivatives (see Table 1.6). The changes in systematic risks are statistically significant in the case of interest rate derivatives and exchange rate derivatives, but not for credit derivatives.

Table 1.6. Difference between Risk Betas: Before and After Use of Financial Derivatives

\begin{tabular}{|c|c|c|c|}
\hline & After Use (A) & Before Use (B) & $\begin{array}{c}\text { Group Difference }(\mathrm{A}-\mathrm{B}) \\
(p \text {-value: } \mathrm{A}=\mathrm{B})\end{array}$ \\
\hline \multicolumn{4}{|c|}{ Panel A: New Users of Interest Rate Derivatives } \\
\hline$\beta_{\text {Market }}$ & $\begin{array}{c}0.894 * * * \\
(39.13)\end{array}$ & $\begin{array}{c}0.408 * * * \\
(20.51)\end{array}$ & $\begin{array}{c}0.486 * * * \\
{[0.000]}\end{array}$ \\
\hline$\beta_{\text {Interest }}$ & $\begin{array}{c}0.623 * * * \\
(14.07)\end{array}$ & $\begin{array}{c}0.266^{* * * *} \\
(6.10)\end{array}$ & $\begin{array}{c}0.357 * * * \\
{[0.000]}\end{array}$ \\
\hline$\beta_{\text {Exchange }}$ & $\begin{array}{c}0.163^{* *} \\
(2.37)\end{array}$ & $\begin{array}{c}0.128^{*} \\
(1.84)\end{array}$ & $\begin{array}{c}0.035 \\
{[0.614]}\end{array}$ \\
\hline$\beta_{\text {Credit }}$ & $\begin{array}{l}0.142 * * * \\
(7.38)\end{array}$ & $\begin{array}{c}-0.0743 * * * \\
(-3.58)\end{array}$ & $\begin{array}{c}0.2163 * * * \\
{[0.000]}\end{array}$ \\
\hline \multicolumn{4}{|c|}{ Panel B: New Users of Exchange Rate Derivatives } \\
\hline$\beta_{\text {Market }}$ & $\begin{array}{c}0.853 * * * \\
(20.87)\end{array}$ & $\begin{array}{c}0.747 * * * \\
(22.87)\end{array}$ & $\begin{array}{c}0.106 * * * \\
{[0.009]}\end{array}$ \\
\hline$\beta_{\text {Interest }}$ & $\begin{array}{l}0.715^{* * * *} \\
\quad(8.12)\end{array}$ & $\begin{array}{l}0.486 * * * \\
(6.75)\end{array}$ & $\begin{array}{c}0.229 * * * \\
{[0.009]}\end{array}$ \\
\hline$\beta_{\text {Exchange }}$ & $0.549 * * *$ & -0.0185 & $0.568 * * *$ \\
\hline
\end{tabular}


Table 1.6. Difference between Risk Betas: Before and After Use of Financial Derivatives

\begin{tabular}{lccc} 
& After Use (A) & Before Use (B) & $\begin{array}{c}\text { Group Difference (A - B) } \\
(p \text {-value: A = B) }\end{array}$ \\
\hline$\beta_{\text {Credit }}$ & $(4.08)$ & $(-0.17)$ & {$[0.000]$} \\
& $0.196^{* * *}$ & $0.0606^{*}$ & $(1.78)$ \\
\hline Panel C: New Users of Credit Derivatives & $(5.25)$ & $0.814^{* * * *}$ & {$[0.000]$} \\
$\beta_{\text {Market }}$ & $1.039^{* * *}$ & $(26.68)$ & $0.227^{* * *}$ \\
$\beta_{\text {Interest }}$ & $(22.27)$ & $0.535^{* * *}$ & {$[0.000]$} \\
$\beta_{\text {Exchange }}$ & $0.632^{* * *}$ & $(7.62)$ & 0.097 \\
$\beta_{\text {Credit }}$ & $(6.74)$ & 0.0988 & {$[0.299]$} \\
& $0.300^{* *}$ & $(0.96)$ & 0.2012 \\
\hline
\end{tabular}

Note. The $t$ statistics are in parentheses. $* p<0.10, * * p<0.05$, *** $p<0.01$. In the brackets, the $p$-values for the test of equality of coefficients before and after use of financial derivatives are reported.

Source: The individual computation is based on the data from the Center for Research in Security Prices (CRSP) database and Federal Reserve monthly Statistical Releases.

Figures 1.5 through 1.8 depict the average systematic interest rate risk, exchange rate risk, credit risk, market risk exposures, and their $10 \%$ confidence interval for all BHCs and for large and small BHCs. Systematic interest rate risk, credit risk, and market risk exposures of BHCs increased substantially during the global financial crisis. Consistent with Hypothesis 1.3, large BHCs are more exposed to higher systematic interest rate risk, exchange rate risk, credit risk, and market risk exposures than small BHCs, especially during the global financial crisis (see Figures $1.5,1.6,1.7$, and 1.8). In contrast, idiosyncratic risk was lower during the financial crisis and small BHCs are exposed to higher idiosyncratic risk than large BHCs (see Figure 1.9).

\section{Figure 1.5. Interest Rate Risk Sensitivity for Three Separate Sample Groups}

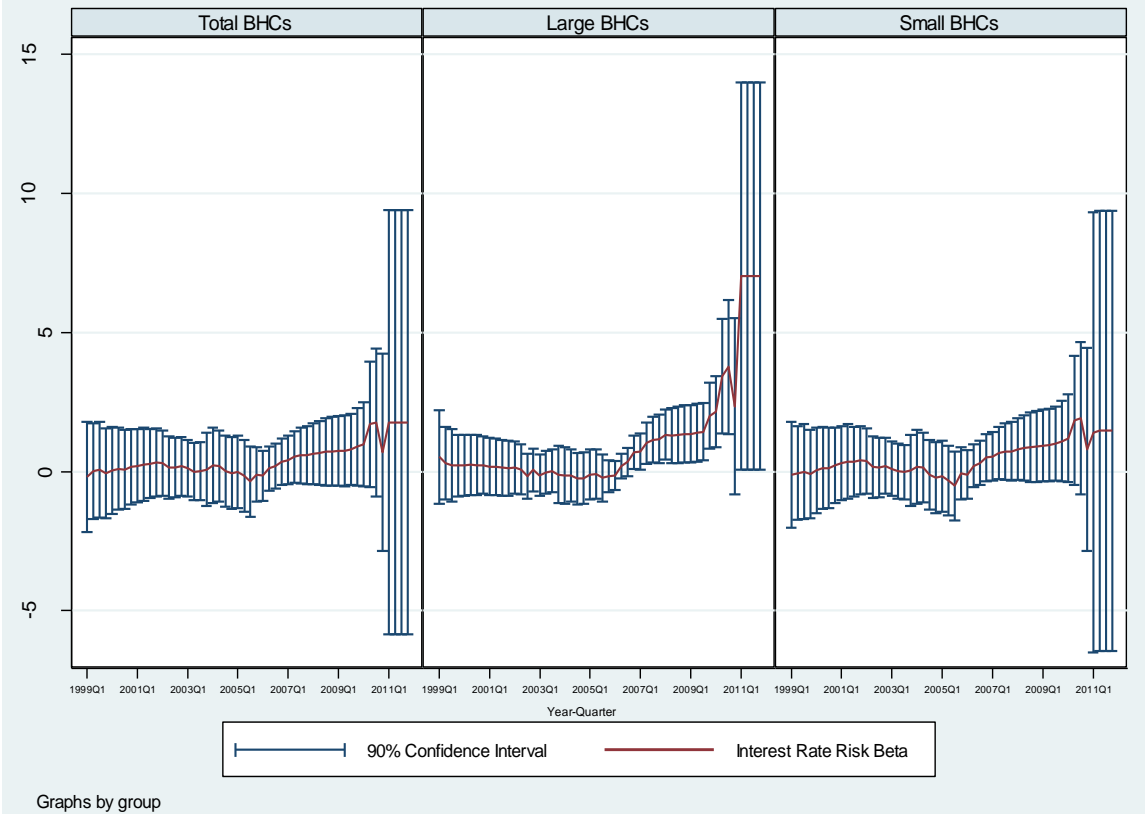

Note. Quarterly Data. Interest rate risk sensitivity is calculated at the midpoint of the four-year rolling window, sample period: 1999:Q1-2012:Q4. 
Figure 1.6. Exchange Rate Risk Sensitivity for Three Separate Sample Groups

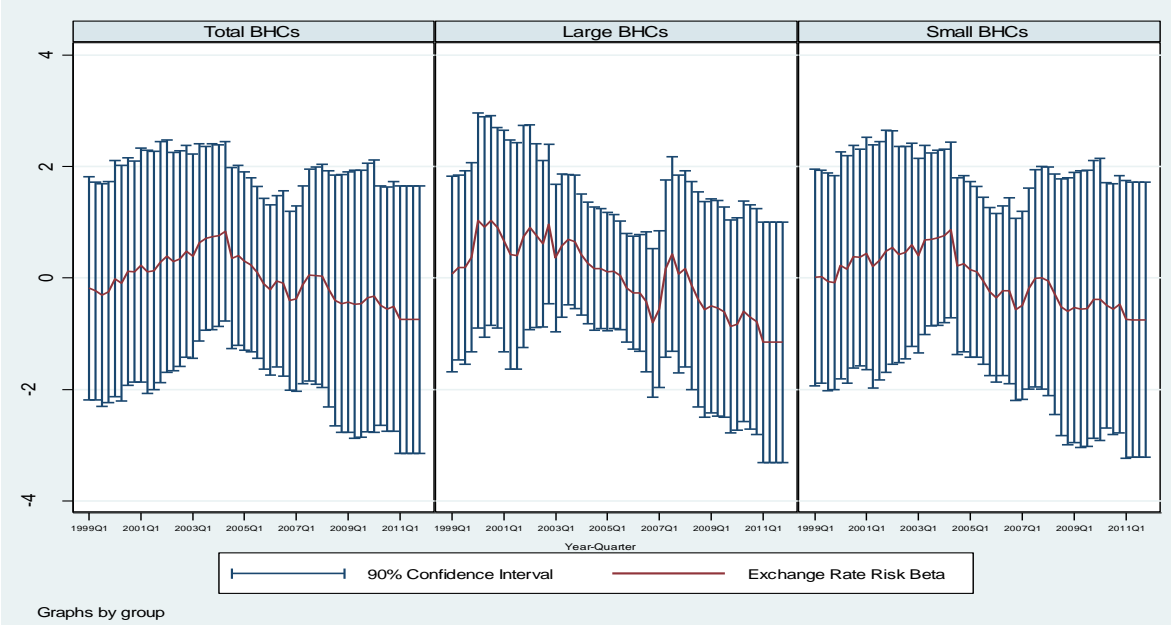

Note. Quarterly Data. Exchange rate risk sensitivity is calculated at the midpoint of the four-year rolling window, sample period: 1999:Q1-2012:Q4.

Figure 1.7. Credit Risk Sensitivity for Three Separate Sample Groups

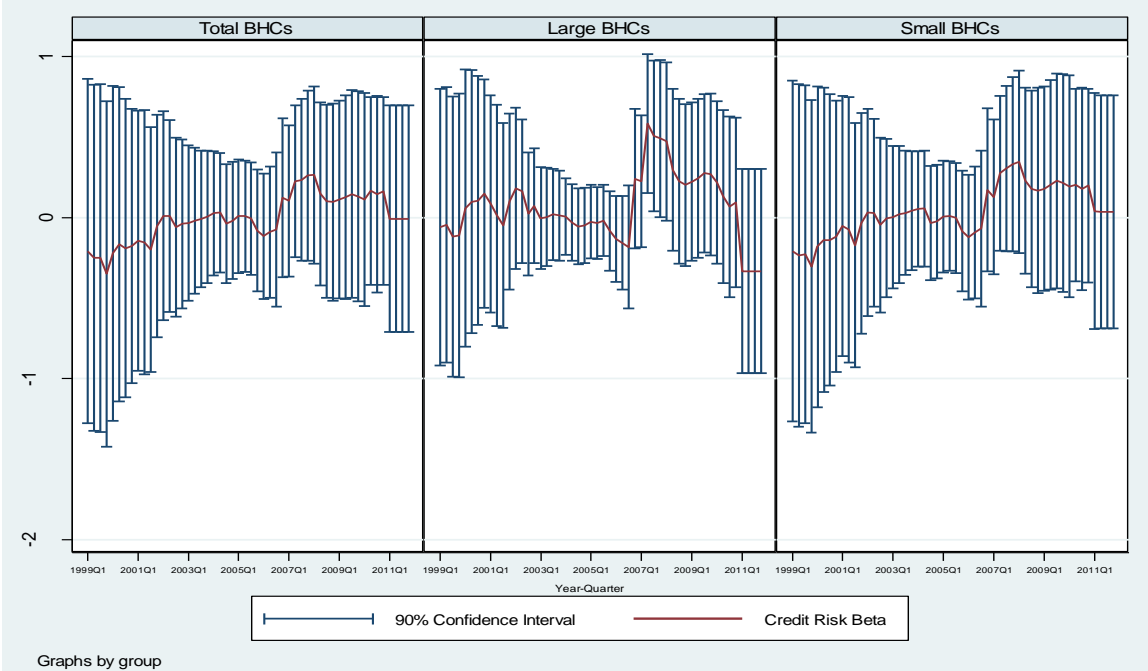

Note. Quarterly Data. Credit risk sensitivity is calculated at the midpoint of the four-year rolling window, sample period: 1999:Q1-2012:Q4.

Figure 1.8. Market Risk Sensitivity for Three Separate Sample Groups

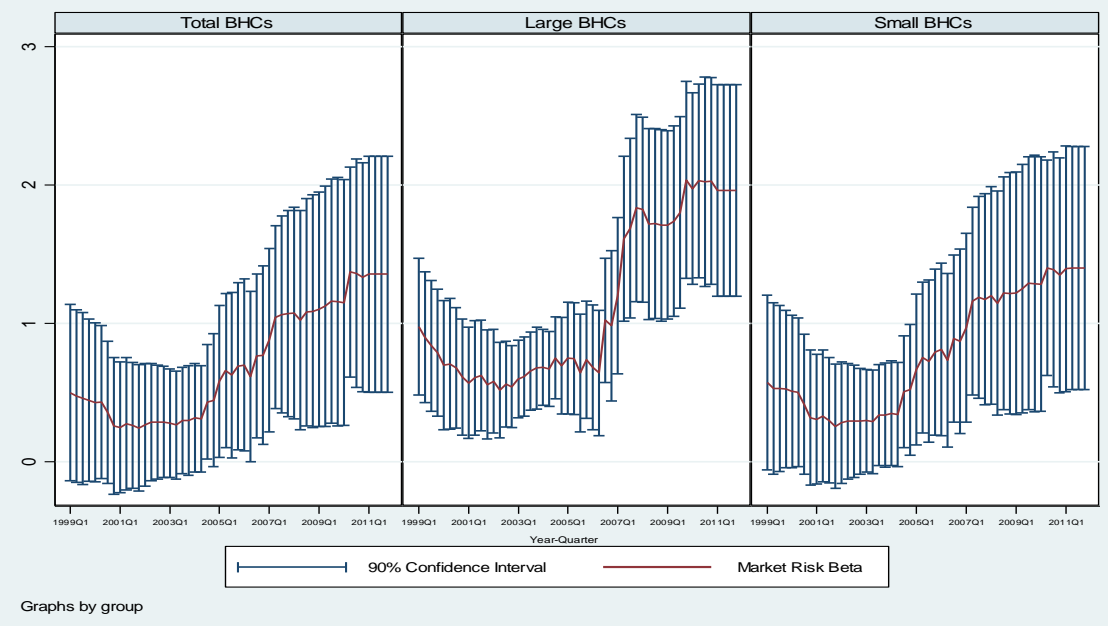

Note. Quarterly Data. Market risk sensitivity is calculated at the midpoint of the four-year rolling window, sample period: 1999:Q1-2012:Q4. 
Figure 1.9. Idiosyncratic Risk $\left(1-R^{2}\right)$ Sensitivity for Three Separate Sample Groups

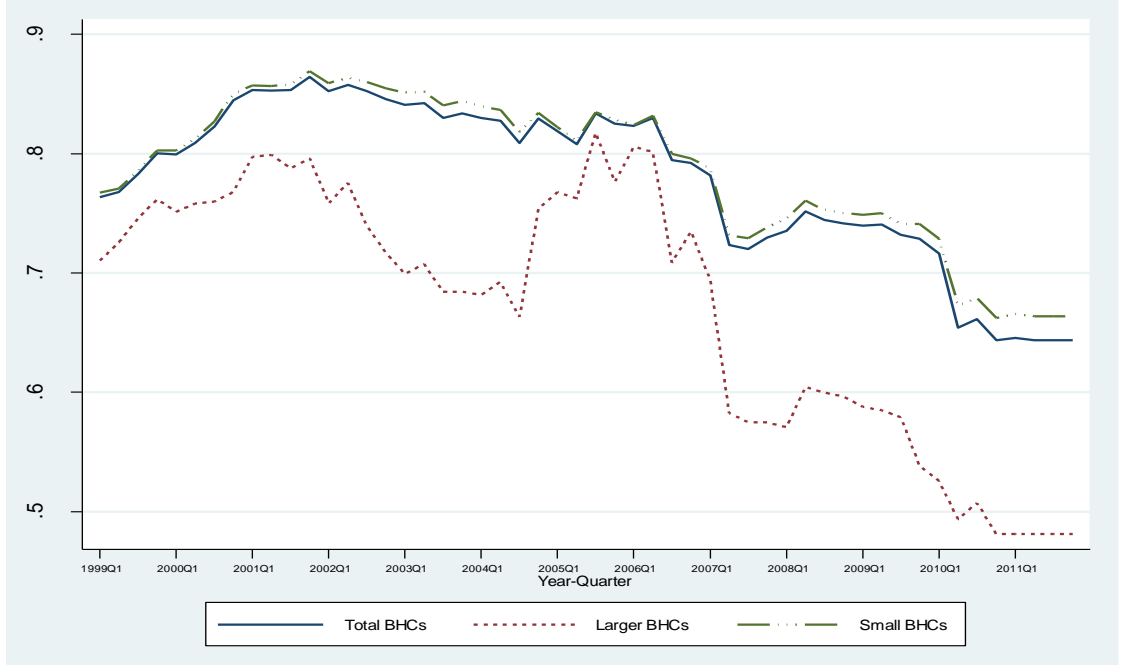

Note. Quarterly Data. Idiosyncratic Risk $\left(1-\mathrm{R}^{2}\right)$ sensitivity is calculated at the midpoint of the four-year rolling window, sample period: 1999:Q1-2012:Q4.

Figures 1.10 through 1.12 provide the first inspection of the impact of financial derivatives (i.e., interest rate derivatives, exchange rate derivatives, and credit derivatives) on systematic risk exposures. BHCs are split into tertiles according to how intensively they use financial derivatives (interest rate derivatives / total assets, exchange rate derivatives / total assets, and credit derivatives / total assets). Figure 1.10 shows that the top tertile of BHCs (i.e., the BHCs with the most intensive use of interest rate derivatives) were exposed to the highest systematic interest rate risk during the global financial crisis. Figures 1.11 and 1.12 show that the tertile of BHCs with the highest use of exchange rate derivatives and credit derivatives were exposed to the highest systematic exchange rate risk and credit risk exposures during the global financial crisis.

Figure 1.10. Interest Rate Risk Sensitivity by Different Size of BHCs (by Interest Rate Derivatives /Total Assets)

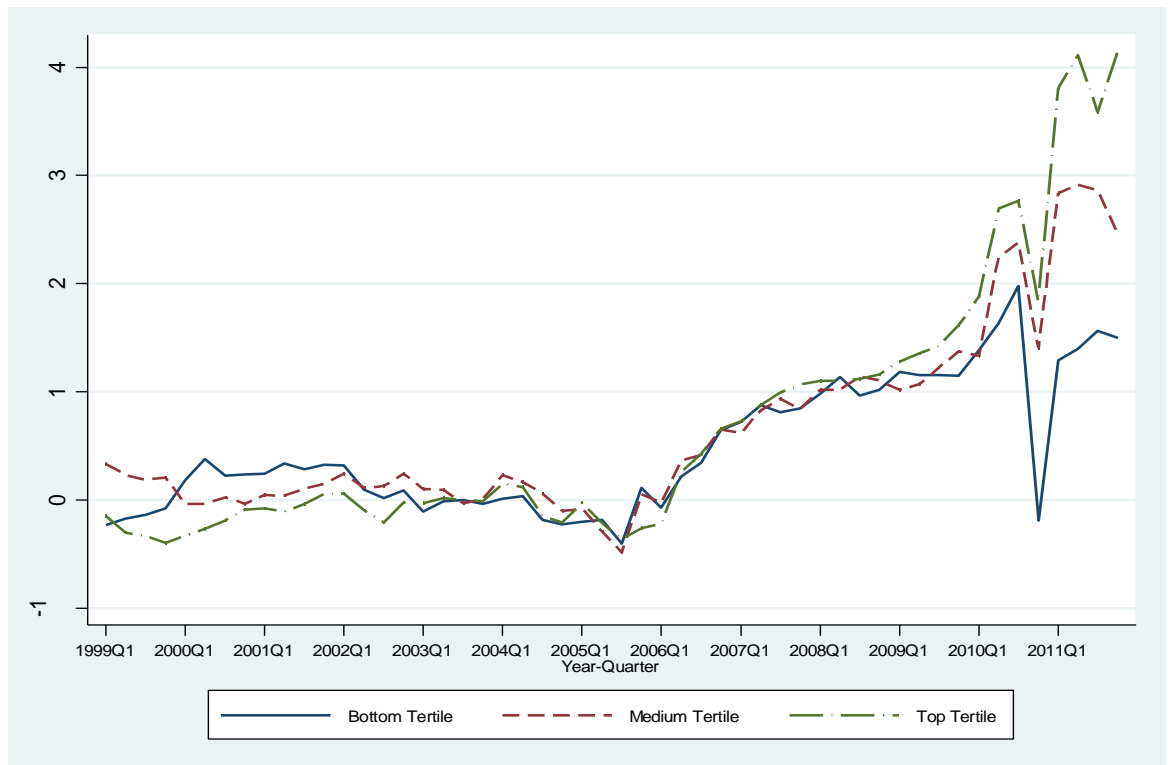

Note. Quarterly Data. Interest rate risk sensitivity is calculated at the midpoint of the four-year rolling window, sample period: 1999:Q1-2012:Q4. 


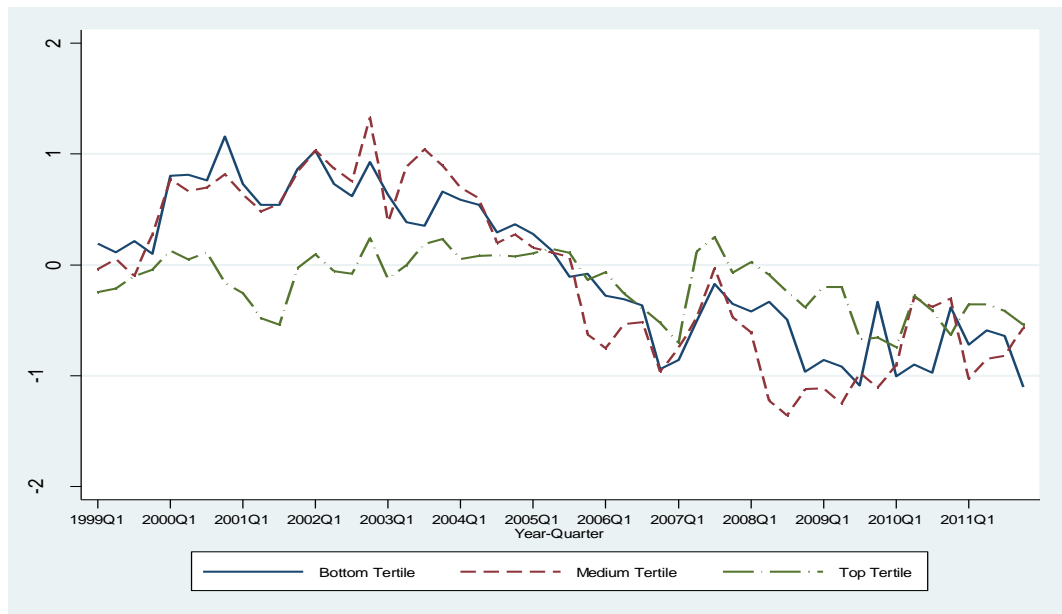

Note. Quarterly Data. Exchange rate risk sensitivity is calculated at the midpoint of the four-year rolling window, sample period: 1999:Q1-2012:Q4.

Figure 1.12. Credit Risk Sensitivity by Different Size of BHCs (by Credit Derivatives / Total Assets)

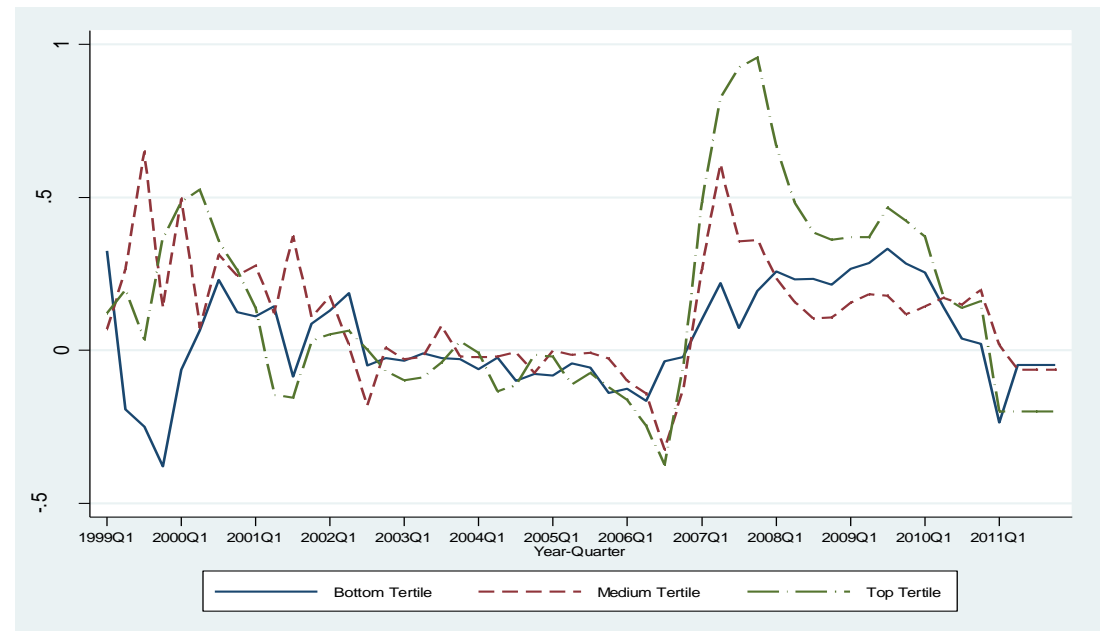

Note. Quarterly Data. Credit risk sensitivity is calculated at the midpoint of the four-year rolling window, sample period: 1999:Q1-2012:Q4.

\subsubsection{Bank-specific determinants of risk betas: initial analysis}

We now present the second-stage regression using the fixed effects panel data regression model based on (1.2), (1.3), and (1.4). ${ }^{18}$ Correlations among the variables used in the second-stage estimation are presented in Table 1.7. Correlations among on-balance-sheet variables and off-balance-sheet variables related to interest rate risk (in Panel A) are generally low, whereas the correlations among BHCs' size and interest rate derivatives variables (interest rate derivatives for trading, interest rate derivatives for hedging, and interest rate derivatives) are higher (above 0.2). This indicates that there is no multicollinearity problem between the on-balance-sheet variables and that a BHC's size is an important driver of the use of interest rate derivatives. The correlations between variables related to exchange rate risk and credit risk are presented in Panels B and C of Table 1.7, respectively.

18 The Hausman test indicates that a fixed effects model should be used rather than a random effects model. 
As a robustness check, we also employ an instrumental-variables estimator. We are concerned about the potential endogeneity of the financial derivatives variables in the fixed effects model. In particular, the changes of risk betas of BHCs may not be driven by financial derivatives alone, but also by other unidentified variables that also affect financial derivatives. For example, BHCs may respond to the increased exposure to trading revenues by employing more financial derivatives. To deal with this endogeneity, we need to find valid instruments that are uncorrelated with the error term but correlated with our dependent variable.

We account for potential endogeneity of financial derivatives variables by instrumenting them with their one-quarter lagged terms, exposure variables from trading revenues (Interest Rate Exposures, Foreign Exchange Exposures, and Credit Exposures), and Income Tax Rate. ${ }^{19}$ Following Baum (2006), we employ the two-stage least squares (2SLS) estimator to obtain a consistent and efficient estimation in the presence of non-i.i.d. errors. Using the Anderson and Rubin (1949) test of the validity of the instruments, the hypothesis that the instruments are not valid is rejected at the 5\% level for all regression models based on (1.2), (1.3), and (1.4). Hansen's J statistics, reported at the bottom of the tables, provide a test for the joint validity of instruments. We never reject the null hypothesis that the overidentifying restrictions are correct. In addition to this, the underidentification test (measured by the Kleibergen-Paap rk LM statistic (Kleibergen and Paap, 2006) and weak identification test (measured by the Cragg-Donald Wald F statistic (Cragg and Donald, 1993), and Kleibergen-Paap Wald rk F statistic (Baum, Schaffer, and Stillman, 2007)) also confirm the validity of instrumental variables. ${ }^{20}$

As an additional robustness check, we use a dynamic panel-data setup to account for potential endogeneity of our dependent variables risk betas in (1.2), (1.3), and (1.4). If lagged risk beta is correlated with the panel-level effects, the estimator may become inconsistent. We use the two-step difference generalized method of moments (GMM) procedure of Arellano and Bond (1991) for the estimation of (1.2), (1.3), and (1.4), in which the lagged levels of the regressor are instruments for the equations in first differences. The Arellano-Bond estimator is useful for obtaining unbiased and efficient estimates in short dynamic panels with lagged endogenous variables as an explanatory variable. Our sample has a large sample dimension and short time dimension. We employ a robust estimator to account for potentially non-i.i.d. errors and to obtain consistent standard error estimates even in the presence of heteroskedasticity or autocorrelation within panels. We use one-quarter lagged risk beta variables and financial derivatives variables as endogenous instruments and exposures variables from trading revenue (Interest Rate Exposures, Foreign Exchange Exposures, and Credit Exposures), Income Tax Rate and all other regressors as exogenous instruments (in line with Roodman (2009)). ${ }^{21}$ We use sets of lags (from 2 to 5) to mitigate the overidentification problem of endogenous instruments.

\footnotetext{
19 Berger and Bouwman (2013) use corporate income tax rates as an instrument for the level of bank capital.

${ }^{20}$ In Appendix A Table A.1, we report the diagnostic tests of instruments used in IV regression.

21 To ensure that interest rate, foreign exchange, and credit exposures are significantly related to financial derivatives and uncorrelated with the risk betas, we have taken the following approach in Table 1.8. In Panel A, the interest rate derivatives are instrumented with foreign exchange and credit exposures. In Panel $\mathrm{B}$, the exchange rate derivatives are instrumented with interest rate and credit exposures. In Panel $\mathrm{C}$, the credit derivatives are instrumented with interest rate and foreign exchange exposures.
} 
Table 1.7. Correlation Between On-and Off-balance-Sheet BHCs'Specific Variables

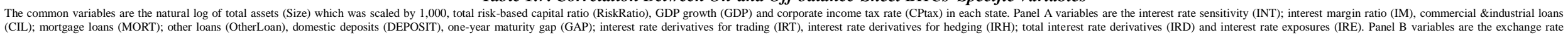

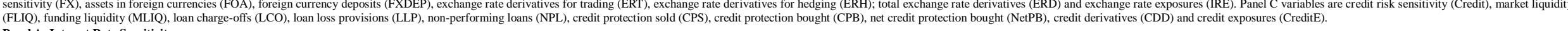
INT IM
$\mathrm{CII}$
MORT
OtherLoar
DEPOSIT
GAP
Size
RiskRatio CPTax
IRT
Cris
IR
GDPgrowth

$\begin{array}{llll}\text { IM } & -0.00735 & 1 & \\ \text { CL } & -0.00660 & 0.0955 * * * & 1 \\ \text { MORT } & 0.0106 & 0.0848 * * * & -0.289 * * *\end{array}$

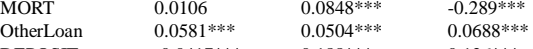

$-0.0417 * * * \quad 0.188$

$\begin{array}{llll} & 0.164 * * * & -0.0124 * * & -0.0447 * * \\ \text { Size } & -0.100 * * & 0.0135\end{array}$

$\begin{array}{llll}\text { RiskRatio } & 0.00710 & -0.00702 & 0.135^{* * * *} \\ \text { CPTat } & -0.0648^{* * * *}\end{array}$

$\begin{array}{lll}\text { IRT } & -0.00654 & -0.04654 * * \\ \text { IRT } & -0.094 * *\end{array}$

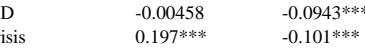

$\begin{array}{lll}\text { IRE } & -0.0171 * & -0.0264 * \\ \text { GDPgrowth } & -0.0935 * * * & 0.102 * * * \\ \end{array}$

Panel B: Exchange Rate Sensitivity

$-0.0382 * * *$

1
$-0.510^{* * * *}$
$0.417^{* * * *}$

$0.417 * * * *-0.172 * * *$

$\begin{array}{ll}-0.0848 * * * & -0.00528 \\ -0.441 * * * & 0.348 *\end{array}$

$-0.0871^{* * *}-0.0135 *-0.118 * * *$

$0.0722^{* * *}$

$\begin{array}{ll}-0.0499^{* * * *} & 0.0580^{* * *} \\ -0.235^{* * *} & 0.0792^{* * * *}\end{array}$

$0.227 * * *-0.167 * * *$

$-0.167 * * * *$
$0.0296 * * *$
$0.0787 * * * *$

$0.0455^{* * * *}$

$-0.354 * * 216 * *$
$-0.369 * * *$

0.00474

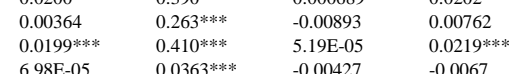

\begin{tabular}{ll}
$0.0363 * * * *$ & -0.00427 \\
$0.139 * * *$ & $0.0287 * *$ \\
\hline
\end{tabular}

Size

RiskRatio

CPTax

ERT

ERH

ERD

FXDEP Siz

1
-0.00326

$0.405^{* * *}$

\begin{tabular}{ll}
-0.0035 & -0.00326 \\
$0.0420^{* * * *}$ & $0.0350^{* * * *}$ \\
\hline & $0.0210 * * 2$
\end{tabular}

${ }^{1} .0149 *$

$\begin{array}{ll}0.421 * * * & 0.0038 \\ 0.211^{* * * *} & 0.0124\end{array}$

$0.0597^{* * * *}$

$\begin{array}{ll}0.427 * * * 36 * * & 0.00453 \\ 0.351 * * * * & -0.00427 \\ 0.00195 & 0.0058\end{array}$

$0.351^{* * * *}$

0.00195
0.00584

$\begin{array}{lll}0.0462 * * * & 0.212^{* * *} & 1 \\ 0.0615^{* * *} & 0.999 * * * & 0.254^{* * *}\end{array}$

$\begin{array}{lll}0.0615 * * * & 0.999 * * * & 0.254 * * * \\ -0.0067 & -0.0114 * & -0.0212 \\ -0.02 * 2 * & 0.027 * * & 0.028 * *\end{array}$

$\begin{array}{lll}0.0802 * * * & 0.627 * * * & -0.00212 \\ -0.00248 & 0.0174 * * & 0.248 * 0 \\ & & \end{array}$

$-0.0114^{*}$

$-0.181^{* * *} \quad-0.0241^{* * *}$

$\begin{array}{lll} & 0.0238 * * * & 0.578 * \\ \text { DPgrowth } & 0.0588 * * * & 0.00757\end{array}$

FLIQ

NPL

LCO

LLP

Size

RiskRatio

CPTax

$-0.0224 * *$

CDS

CDB

NePB

CDD

Crisis

CreditE

GDPgrowth

$0.600^{* * * *}$

$\begin{array}{ll}0.00259 & 0.909 * * * * \\ 0.112 * * *\end{array}$

$0.0693 * * *$

$-0.0810^{* * * *}$

$-0.0243^{* * *}$

$-0.0200^{* * *}$

$-0.0239 * * *$

$\begin{array}{ll}0.00349 & 0.00185 \\ 2 & 0.00127\end{array}$

$\begin{array}{lll}0.266^{* * * *} & 0.00528 & 0.00735 \\ 0.198^{* * * *} & 0.000779 & 0.0175^{*} \\ 0 & 0.0036 & 0.0675\end{array}$

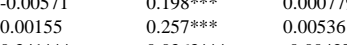

$-0.0239^{* * * *}$
$-0.276^{* * * *}$

$0.175 * * *$
-0.0048

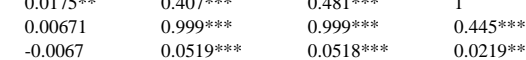

$-0.0321^{* * * *}$

$\begin{array}{lll}0.0511^{* * * *} & 0.0219 * *\end{array}$

$0.398 * * * *$
$-0.0218 * * * *$

$0.0519^{* * * *}$

$*$ factor model using data from Center for
$* p<0.10, * * * 0.05, * * * p<0.01$. 
Table 1.8 provides the regression results. Panel A in Table 1.8 shows that the use of Interest Rate Derivatives is positively and significantly (at 1\%) associated with systematic interest rate risk exposure for the total sample and two subsamples. This indicates that interest rate derivatives are mainly used speculatively rather than for a hedging purpose: they may be used for fee-generating business such as trading. This result is consistent with previous studies (e.g., Hirtle, 1997; Reichert and Shyu, 2003; Yong et al., 2009).

The results from Panel A show that C\&I Loans, Size, and Capital Ratio are positively and significantly associated with systematic interest rate risk for the total sample. This is consistent with previous findings by Elyasiani and Mansur (1998, 2004), Saporoschenko (2002), Reichert and Shyu (2003), and Faff, Hodgson, and Kremmer (2005), and indicates that especially large BHCs with higher lending activities are exposed to higher systematic interest rate risk exposure. GAP Ratio is negatively associated with systematic interest rate risk for the total sample and two subsamples. GDP Growth has a significant and negative impact on systematic interest rate risk exposure for small BHCs.

Panel B in Table 1.8 analyzes systematic exchange rate risk of BHCs. Exchange Rate Derivatives are positively and significantly (at $1 \%$ ) associated with systematic exchange rate risk for the total sample, large BHCs, and small BHCs. This demonstrates that BHCs are exposed to higher systematic exchange rate risk when they use more exchange rate derivatives. In addition, the economic impact of exchange rate derivatives on systematic exchange rate risk is more pronounced for small BHCs than for large BHCs, indicating that the speculative purpose of exchange rate derivatives is stronger for small BHCs.

Panel B in Table 1.8 also shows that Foreign Exchange Deposits are positively associated with systematic exchange rate risk exposure for the total sample and small BHCs, whereas Assets in Foreign Currencies are negatively associated with systematic exchange rate risk exposure for large BHCs but positively for small BHCs. The explanation may be that small BHCs are restricted by their small size and international business, and can hardly combine foreign exchange deposit-taking with lending in the same foreign currencies and hedge against systematic exchange rate risk exposure as large BHCs. We also see that Size is negatively and significantly associated with systematic exchange rate risk exposure. This may suggest that large BHCs in particular match assets in foreign currencies and foreign exchange deposits in order to lower systematic exchange rate risk exposures.

Panel C of Table 1.8 depicts the systematic credit risk exposure of BHCs. The use of Credit Derivatives is positively and significantly related to systematic credit risk exposure for the total sample, large BHCs, and small BHCs. The relationship is stronger for large BHCs than for the total sample or small BHCs. This may indicate that especially large BHCs use credit derivatives predominantly not to hedge, but to further expose themselves towards higher systematic credit risk exposure.

Market Liquidity is negatively and significantly (at 1\%) associated with systematic credit risk 
exposure. The explanation may be that liquid funds help BHCs mitigate their exposure to systematic credit risk. Non-Performing Loans and Loan Charge-Offs are negatively associated with systematic credit risk exposure. Loan Loss Provisions are positively and significantly associated with systematic credit risk exposure. Size and GDP Growth are positively and statistically significantly (at $1 \%$ ) related to systematic credit risk exposure for the total sample and two subsamples.

In short, the use of interest rate derivatives, exchange rate derivatives, and credit derivatives is positively and significantly related to systematic interest rate, exchange rate, and credit risk. This points to the positive relationship between financial derivatives and risks in Hypothesis 1.1. 
Table 1.8. Determinants of Interest Rate, Exchange Rate, and Credit Risk Betas

Panel A: Interest Rate Risk Beta

\author{
Interest Margin
}

C\&I Loans

Mortgage Loans

Other Loans

Domestic Deposits

GAP Ratio

Size

Capital Ratio

GDP Growth

Interest Rate Derivatives

L.Interest Rate Risk Beta

$N$

Adjusted-R2

$\mathrm{AR}(1)$

$\operatorname{AR}(2)$

Hansen J Statistic ( $p$-value)

Number of Instruments

Panel B: Exchange Rate Risk Beta

Assets in Foreign Currencies

Foreign Exchange Deposits

Size

Capital Ratio

GDP Growth

Exchange Rate Derivatives
Total BHCs

Large BHCs

Small BHCs

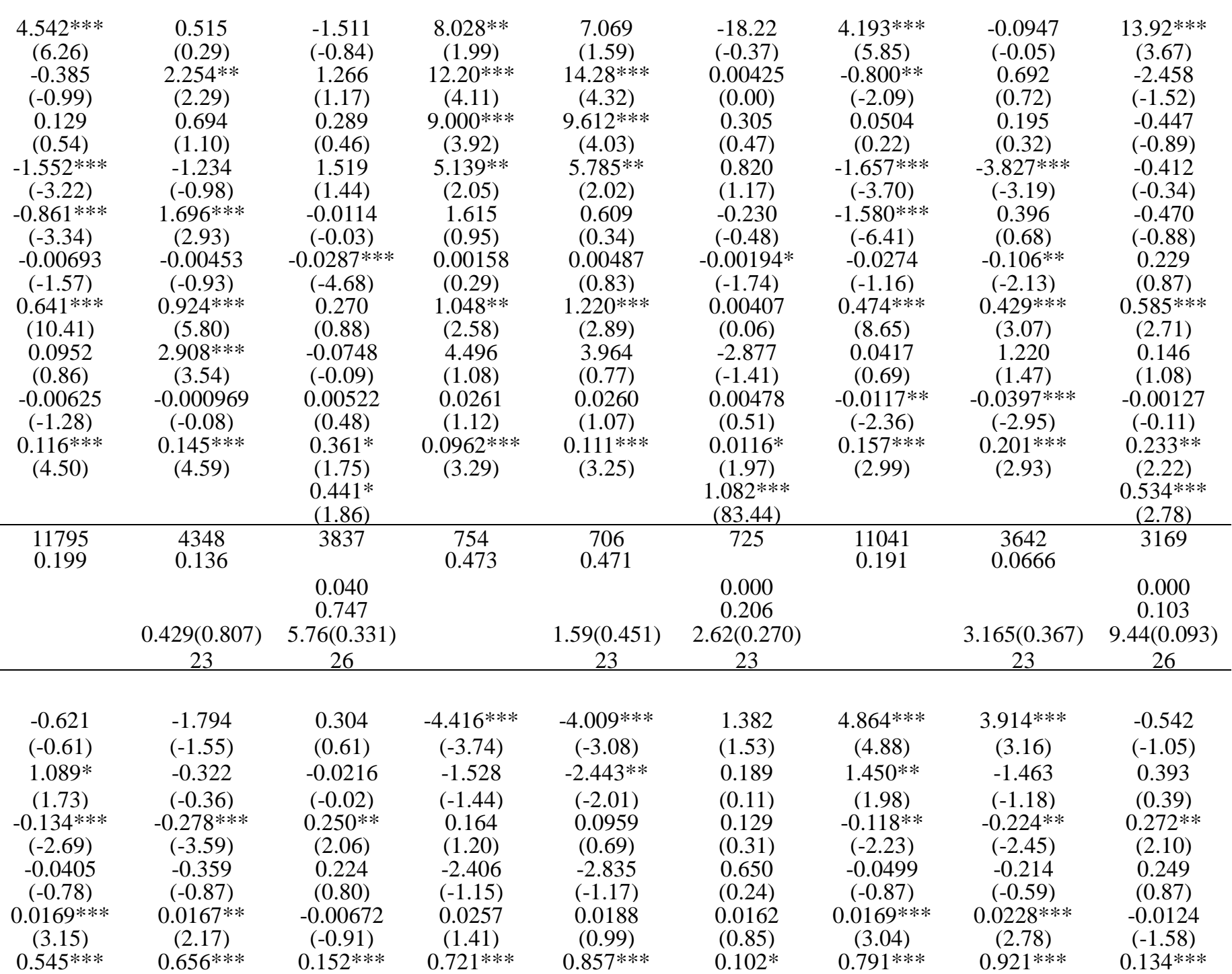


Table 1.8. Determinants of Interest Rate, Exchange Rate, and Credit Risk Betas

L. Exchange Rate Risk Beta Total BHCs

\begin{tabular}{|c|c|c|c|c|c|c|c|c|c|}
\hline & & & $(10.68)$ & & & $(4.08)$ & & & $(10.42)$ \\
\hline$N$ & 11803 & 4349 & 3835 & 759 & 711 & 682 & 11044 & 3638 & 3153 \\
\hline Adjusted-R2 & 0.165 & 0.205 & & 0.404 & 0.404 & & 0.160 & 0.193 & \\
\hline $\mathrm{AR}(1)$ & & & 0.000 & & & 0.036 & & & 0.000 \\
\hline $\mathrm{AR}(2)$ & & & 0.476 & & & 0.131 & & & 0.541 \\
\hline Hansen J Statistic ( $p$-value) & & $4.97(0.147)$ & $1.75(0.626)$ & & $0.120(0.942)$ & $2.70(0.746)$ & & $4.503(0.105)$ & $1.99(0.737)$ \\
\hline Number of Instruments & & 19 & 20 & & 19 & 22 & & 19 & 21 \\
\hline \multicolumn{10}{|l|}{ Panel C: Credit Risk Beta } \\
\hline Market Liquidity & $\begin{array}{c}-0.511 * * * \\
(-9.38)\end{array}$ & $\begin{array}{c}-0.440 * * * \\
(-5.48)\end{array}$ & $\begin{array}{c}-0.0904 \\
(-1.21)\end{array}$ & $\begin{array}{l}-0.253 \\
(-1.11)\end{array}$ & $\begin{array}{c}-0.438 * \\
(-1.95)\end{array}$ & $\begin{array}{l}-0.274 \\
(-0.05)\end{array}$ & $\begin{array}{c}-0.518 * * * \\
(-9.23)\end{array}$ & $\begin{array}{c}-0.463 * * * \\
(-5.53)\end{array}$ & $\begin{array}{l}0.118 \\
(1.11)\end{array}$ \\
\hline Funding Liquidity & $\begin{array}{c}-0.388 * * * \\
(-2.94)\end{array}$ & $\begin{array}{c}-0.661 * * * \\
(-4.02)\end{array}$ & $\begin{array}{c}0.0333 \\
(0.32)\end{array}$ & $\begin{array}{c}-1.562 * * * \\
(-3.59)\end{array}$ & $\begin{array}{c}-1.601 * * * \\
(-3.76)\end{array}$ & $\begin{array}{c}13.63 * * \\
(2.13)\end{array}$ & $\begin{array}{c}-0.346^{* *} \\
(-2.48)\end{array}$ & $\begin{array}{c}-0.545 * * * \\
(-3.02)\end{array}$ & $\begin{array}{c}-0.0266 \\
(-0.21)\end{array}$ \\
\hline Non-Performing Loans & $\begin{array}{c}-4.717 * * * \\
(-11.39)\end{array}$ & $\begin{array}{c}-3.149 * * * \\
(-6.25)\end{array}$ & $\begin{array}{l}-0.510 \\
(-1.56)\end{array}$ & $\begin{array}{c}-11.42 * * * \\
(-7.45)\end{array}$ & $\begin{array}{c}-12.54 * * * * \\
(-8.51)\end{array}$ & $\begin{array}{l}-39.86 \\
(-0.07)\end{array}$ & $\begin{array}{c}-4.269 * * * \\
(-10.17)\end{array}$ & $\begin{array}{c}-1.574 * * * \\
(-3.23)\end{array}$ & $\begin{array}{l}0.231 \\
(0.75)\end{array}$ \\
\hline Loan Charge-Offs & $\begin{array}{c}-5.922 * * * \\
(-2.68)\end{array}$ & $\begin{array}{c}-4.519 * \\
(-1.94)\end{array}$ & $\begin{array}{l}-0.267 \\
(-0.26)\end{array}$ & $\begin{array}{c}-17.73 * * * \\
(-3.13)\end{array}$ & $\begin{array}{c}-23.53 * * * \\
(-5.16)\end{array}$ & $\begin{array}{l}-41.32 \\
(-1.22)\end{array}$ & $\begin{array}{c}-3.878^{*} \\
(-1.65)\end{array}$ & $\begin{array}{l}-0.766 \\
(-0.33)\end{array}$ & $\begin{array}{l}0.148 \\
(0.12)\end{array}$ \\
\hline Loan Loss Provisions & $\begin{array}{c}6.488 * * * \\
(3.19)\end{array}$ & $\begin{array}{c}4.983 * * \\
(2.21)\end{array}$ & $\begin{array}{l}0.334 \\
(0.31)\end{array}$ & $\begin{array}{c}12.56 * * * \\
(2.70)\end{array}$ & $\begin{array}{c}16.66^{* * * *} \\
(3.91)\end{array}$ & $\begin{array}{c}80.43 * * \\
(2.82)\end{array}$ & $\begin{array}{c}5.592 * * * \\
(2.60)\end{array}$ & $\begin{array}{l}2.595 \\
(1.15)\end{array}$ & $\begin{array}{c}2.839 * * \\
(2.35)\end{array}$ \\
\hline Size & $\begin{array}{c}0.0496 \text { **** } \\
(2.83)\end{array}$ & $\begin{array}{c}-0.00236 \\
(-0.09)\end{array}$ & $\begin{array}{c}-0.0175 \\
(-0.58)\end{array}$ & $\begin{array}{c}0.207 * * * \\
(4.14)\end{array}$ & $\begin{array}{l}0.277 * * * \\
(5.99)\end{array}$ & $\begin{array}{l}-0.388 \\
(-0.72)\end{array}$ & $\begin{array}{c}0.0658 * * * \\
(3.60)\end{array}$ & $\begin{array}{l}0.0267 \\
(0.93)\end{array}$ & $\begin{array}{c}0.00513 \\
(0.11)\end{array}$ \\
\hline Capital Ratio & $\begin{array}{c}-0.00646 \\
(-0.69)\end{array}$ & $\begin{array}{c}-0.0208 \\
(-0.24)\end{array}$ & $\begin{array}{c}0.126 * * \\
(2.05)\end{array}$ & $\begin{array}{c}2.162 * * * \\
(3.03)\end{array}$ & $\begin{array}{c}1.720 * * \\
(2.16)\end{array}$ & $\begin{array}{c}-33.13^{*} \\
(-1.82)\end{array}$ & $\begin{array}{c}-0.00130 \\
(-0.11)\end{array}$ & $\begin{array}{c}0.0688 \\
(0.78)\end{array}$ & $\begin{array}{c}0.0879 \\
(1.00)\end{array}$ \\
\hline GDP Growth & $\begin{array}{c}0.00617 * * * \\
(3.40)\end{array}$ & $\begin{array}{c}0.00612 * * \\
(2.23)\end{array}$ & $\begin{array}{c}0.00575 * * \\
(2.04)\end{array}$ & $\begin{array}{c}0.0161 * * \\
(2.41)\end{array}$ & $\begin{array}{l}0.0164 * * \\
(2.54)\end{array}$ & $\begin{array}{c}-0.0677 \\
(-1.63)\end{array}$ & $\begin{array}{c}0.00520 * * * \\
(2.80)\end{array}$ & $\begin{array}{c}0.00439 \\
(1.52)\end{array}$ & $\begin{array}{c}0.00694 * \\
(1.67)\end{array}$ \\
\hline Credit Derivatives & $\begin{array}{c}0.0761 * * * \\
(4.73)\end{array}$ & $\begin{array}{c}0.0738 * * * \\
(4.35)\end{array}$ & $\begin{array}{c}0.0264 * * \\
(2.51)\end{array}$ & $\begin{array}{c}0.103 * * * \\
(5.88)\end{array}$ & $\begin{array}{c}0.0960 * * * \\
(5.77)\end{array}$ & $\begin{array}{c}0.995 * * * \\
(3.65)\end{array}$ & $\begin{array}{c}0.0708 * \\
(1.76)\end{array}$ & $\begin{array}{l}0.101 \\
(1.41)\end{array}$ & $\begin{array}{c}0.0466 * * * \\
(3.32)\end{array}$ \\
\hline L. Credit Risk Beta & & & $\begin{array}{c}-0.683 * * * \\
(-7.34)\end{array}$ & & & $\begin{array}{c}0.0664 \\
(0.19) \\
\end{array}$ & & & $\begin{array}{c}0.901 * * * \\
(7.31) \\
\end{array}$ \\
\hline$N$ & 10984 & 4114 & 3655 & 697 & 667 & 646 & 10287 & 3447 & 3009 \\
\hline Adjusted-R2 & 0.163 & 0.218 & & 0.594 & 0.620 & & 0.149 & 0.168 & \\
\hline $\operatorname{AR}(1)$ & & & 0.000 & & & 0.002 & & & 0.000 \\
\hline $\operatorname{AR}(2)$ & & & 0.785 & & & 0.480 & & & 0.127 \\
\hline Hansen J Statistic ( $p$-value) & & $1.166(0.558)$ & $1.94(0.585)$ & & $0.171(0.679)$ & $6.91(0.960)$ & & $3.365(0.339)$ & $3.34(0.342)$ \\
\hline Number of Instruments & & 21 & 22 & & 21 & 34 & & 21 & 22 \\
\hline Estimation Method & FE & IV & GMM & $\mathrm{FE}$ & IV & GMM & FE & IV & GMM \\
\hline
\end{tabular}

Note: The dependent variable in each Panel is our estimates of risk beta of each BHC $i$ at the start time $t$ of four-year rolling window regression in the first-stage. We weight each observation by the inverse of the standard error of beta coefficients in the first-stage estimation. The regressions included bank-specific fixed effects and yearly dummy variables. Heteroskedasticity-consistent standard errors are used and $t$ statistics are reported in parentheses. ${ }^{*} p<0.10,{ }^{* * *} p<0.05,{ }^{* * * *} p<0.01$.

Source: Financial data is from FR Y-9C; Risk betas are computed from the four-factor model using data from Center for Research in Security Prices (CRSP) database and Federal Reserve monthly. 


\subsubsection{Purposes of financial derivatives and systematic risk exposures}

To further depict the relationship between risks and financial derivatives, we analyze how financial derivatives for trading and financial derivatives for hedging affect systematic interest rate risk, exchange rate risk, and credit risk exposures, and we analyze the impact of bank capital ratio and bank size. ${ }^{22}{ }^{23}$

Panel A in Table 1.9 shows that the positive relationship between financial derivatives and systematic interest rate risk is more pronounced for BHCs with a higher total capital ratio and tier 1 ratio. Interest Rate Derivatives for Trading and Interest Rate Derivatives for Hedging are both positively and significantly related to systematic interest rate risk (column 5). For BHCs with higher Capital Ratio, Tier 1 Ratio, and Size, the positive impact of Interest Rate Derivatives for Trading on systematic interest rate risk becomes stronger, whereas the impact of Interest Rate Derivatives for Hedging weakens (or is insignificant).

Panel B in Table 1.9 shows that the positive relationship between exchange rate derivatives and systematic exchange rate risk is more pronounced for larger BHCs. Exchange Rate Derivatives for Trading and Exchange Rate Derivatives for Hedging are both significantly and positively related to systematic exchange rate risk. For large BHCs and for BHCs with higher Capital Ratio and Tier 1 Ratio, the positive relationship between Exchange Rate Derivatives for Trading and systematic exchange rate risk becomes more pronounced, whereas the positive relationship between Exchange Rate Derivatives for Hedging and systematic exchange rate risk weakens (or the impact is insignificant).

Panel $\mathrm{C}$ in Table 1.9 shows that the positive relationship between credit derivatives and systematic credit risk becomes more pronounced for larger BHCs and for BHCs with lower Capital Ratio and Tier 1 Ratio. Gross Credit Protection and Net Credit Protection Bought are significantly and positively related to systematic credit risk, but this positive relationship becomes less pronounced for BHCs with higher Capital Ratio and Tier 1 Ratio. The positive relationship between Gross Credit Protection and systematic credit risk weakens for larger BHCs (but with low statistical significance), whereas the positive relationship between Net Protection Bought and systematic credit risk becomes more pronounced.

Consistent with our expectations in Hypotheses 1.1a and 1.1b, both financial derivatives for hedging and financial derivatives for trading impact systematic risks of BHCs. We show that this relationship is positive and highly statistically significant. This result suggests that the real

\footnotetext{
22 Bank regulatory reports separate financial derivatives (interest rate, foreign exchange, commodity, and equity derivatives) held for trading purposes and for purposes other than trading, but do not separately report credit derivatives held for trading and for hedging purposes. Hence, similar to Minton, Stulz, and Williamson (2009) and Hirtle (2009), we use net credit protection bought, which is the difference between the notional principal of credit derivatives on which the bank is a beneficiary (Credit Protection Bought) and the notional principal amount of credit derivatives on which the bank is a guarantor (Credit Protection Sold) as a measure of the extent to which BHCs use credit derivatives to hedge credit risk.

${ }^{23}$ We also included the dummy variable SIFI in the regression, but it was dropped from the model due to collinearity.
} 
impact of financial derivatives for hedging on systematic risk exposure is inconsistent with their reported purpose.

Our empirical findings support Hypothesis 1.3, which states that the positive relationship between financial derivatives and risks is stronger for larger BHCs (especially for Exchange Rate Derivatives and Credit Derivatives). We also find empirical support for Hypothesis 1.2, which states that the relationship between financial derivatives and risk is influenced by the BHC's capital strength. The sign of the relationship, however, changes across the types of the financial derivatives. Capital Ratio and Tier 1 Ratio significantly strengthen the positive relationship between Interest Rate Derivatives and systematic interest rate risk, and weaken the positive relation between Credit Derivatives and systematic credit risk. High capital reinforces the positive relationship between financial derivatives for trading and systematic risks, but weakens the positive relationship between financial derivatives for hedging and systematic risk. This may indicate that weakly capitalized banks classify more derivatives as hedging derivatives to be treated more favorably by the regulator, which strengthens the positive relation between financial derivatives for hedging and systematic risk. 
Table 1.9. Determinants of Interest Rate, Exchange Rate, and Credit Risk Betas, and Interaction Terms

Panel A: Interest Rate Risk Beta

Interest Margin

\section{C\&I Loans}

Mortgage Loans

Other Loans

Domestic Deposits

GAP Ratio

Size

Capital Ratio

Tier1 Ratio

GDP Growth

Interest Rate Derivatives

Capital Ratio * Interest Rate Derivatives

Tier1 Ratio * Interest Rate Derivatives

Size * Interest Rate Derivatives

SIFI * Interest Rate Derivatives

Interest Rate Derivatives for Trading

Interest Rate Derivatives for Hedging

Capital Ratio * Interest Rate Derivatives for Trading

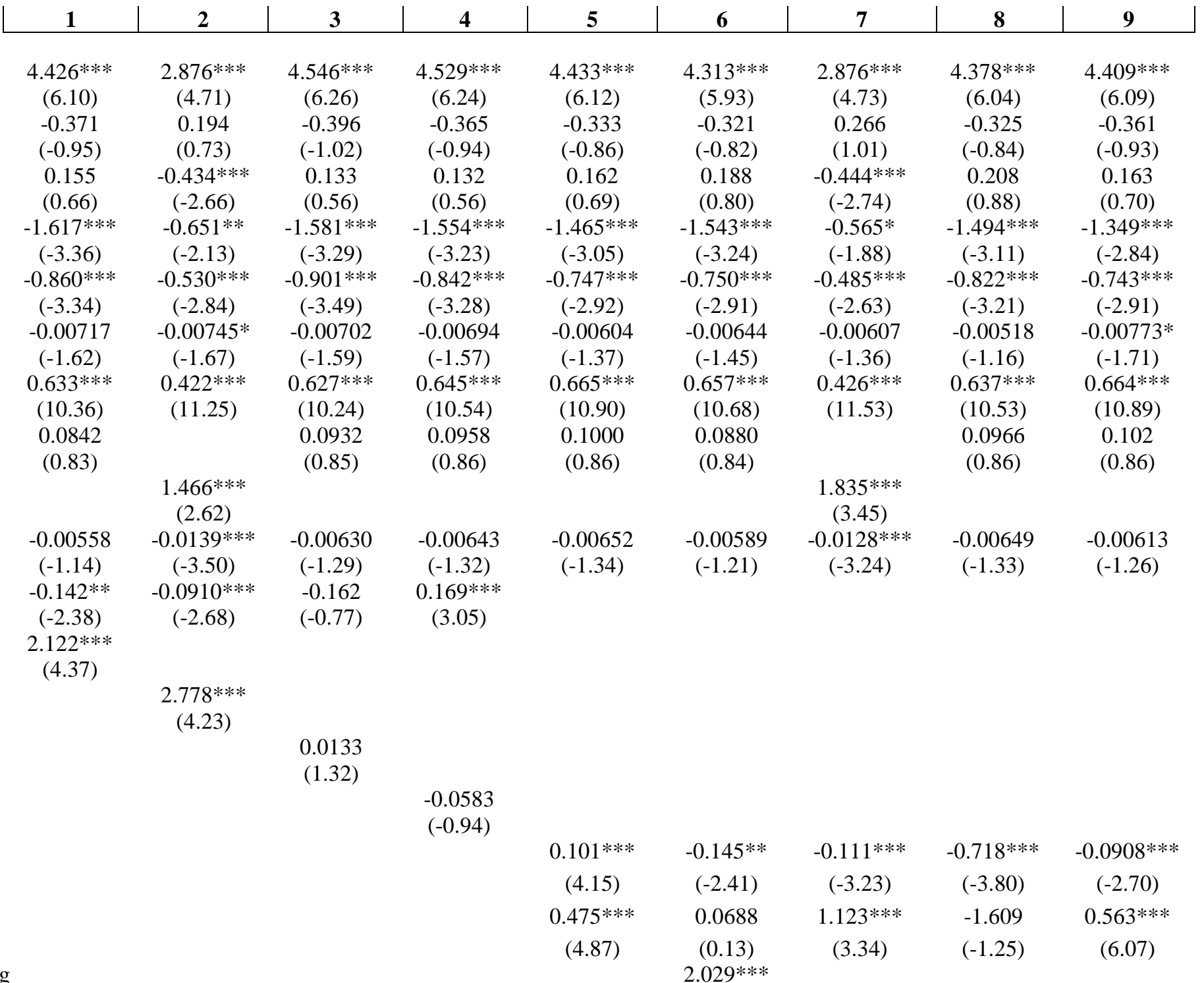


Table 1.9. Determinants of Interest Rate, Exchange Rate, and Credit Risk Betas, and Interaction Terms

\begin{tabular}{|c|c|c|c|c|c|}
$\mathbf{1}$ & $\mathbf{2}$ & $\mathbf{3}$ & $\mathbf{4}$ & $\mathbf{5}$ & $\mathbf{6}$ \\
\hline
\end{tabular}

Capital Ratio * Interest Rate Derivatives for Hedging

3.009

Tier1 Ratio * Interest Rate Derivatives for Trading

$(0.78)$

Tier1 Ratio*Interest Rate Derivatives for Hedging

Size * Interest Rate Derivatives for Trading

Size * Interest Rate Derivatives for Hedging

SIFI * Interest Rate Derivatives for Trading

\begin{tabular}{l} 
SIFI * Interest Rate Derivatives for Hedging \\
\hline$N$
\end{tabular}

$\mathrm{N}$ Adjusted-R2

Panel B: Exchange Rate Risk Beta

Assets in Foreign Currencies

Foreign Exchange Deposits

Size

Capital Ratio

Tier1 Ratio

GDP Growth

Exchange Rate Derivatives

Capital Ratio * Exchange Rate Derivatives

Tier1 Ratio * Exchange Rate Derivatives

Size * Exchange Rate Derivatives

\author{
$2.861 * * *$
$(4.15)$ \\ $(4.15)$
$-7.456^{*}$ \\ $(-1.92)$
}

$0.0389 * * *$

$(4.19)$

0.127

(1.58)

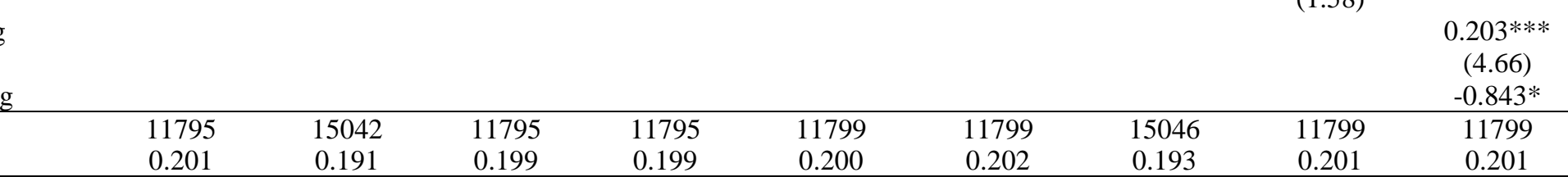

$\begin{array}{lllllllll}-0.658 & -0.495 & -0.617 & -0.747 & -0.686 & -2.136^{*} & -1.622 & -0.850 & -1.011\end{array}$

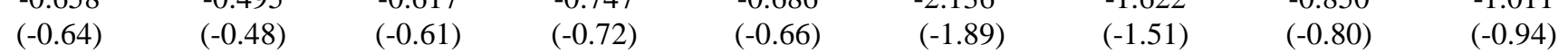

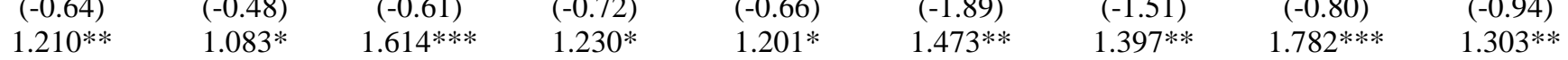

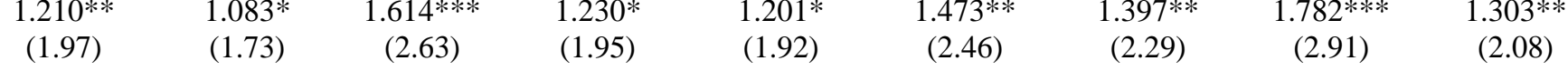

$\begin{array}{lllllllll}-0.136 * * * & -0.143 * * * & -0.150 * * * & -0.132 * * * & -0.140 * * * & -0.143 * * * & -0.157 * * * & -0.159 * * * & -0.141 * * *\end{array}$

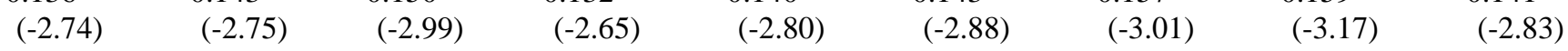

$\begin{array}{ccccccc}-0.0438 & -0.0458 & -0.0406 & -0.0416 & -0.0440 & -0.0477 & -0.0421 \\ (-0.81) & (-0.83) & (-0.78) & (-0.79) & (-0.82) & (-0.84) & (-0.79)\end{array}$

$\begin{array}{ll}-0.420 & -0.640 \\ (-0.88) & (-1.30)\end{array}$

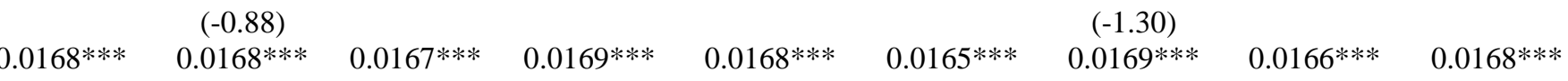

$\begin{array}{lllllll}(3.13) & (3.14) & (3.13) & (3.17) & (3.15) & (3.08) & \text { (3.14) }\end{array}$

$0.381 * * * \quad 0.472 * * * \quad-1.697 * * \quad 0.796 * * *$

$\begin{array}{llll}(2.60) & (3.33) \quad(-2.36) \quad(5.47)\end{array}$

1.012

(1.35)

1.052

(0.64)

$0.115^{* * *}$

(3.15) 
Table 1.9. Determinants of Interest Rate, Exchange Rate, and Credit Risk Betas, and Interaction Terms

SIFI * Exchange Rate Derivatives

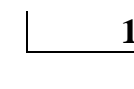

2

Exchange Rate Derivatives for Trading

Exchange Rate Derivatives for Hedging

Capital Ratio * ExchangeRate Derivatives for Trading

CapitalRatio*Exchange RateDerivatives forHedging

Tier1 Ratio * Exchange Rate Derivatives for Trading

Tier1 Ratio * Exchange Rate Derivatives for Hedging

Size * Exchange Rate Derivatives for Trading

Size * Exchange Rate Derivatives for Hedging

SIFI * Exchange Rate Derivatives for Trading

3

4

$(-1.79)$ 5

6

$\begin{array}{ccccc}0.501 * * * & 0.248 * & 0.328 * * & -1.717 * * & 0.672 * * * \\ (5.66) & (1.75) & (2.43) & (-2.13) & (4.42) \\ 2.316 * * * & 11.41 * * * & 8.873 * * * & -13.31 & 2.073 * * *\end{array}$
$(3.78)$
$1.335^{*}$
(1.83)

(5.18)

$(-0.94)$

(4.44)

$-49.39 * * *$

$(-3.56)$

$$
\begin{gathered}
2.096 \\
(1.32) \\
-91.64 * * * \\
(-4.25)
\end{gathered}
$$

0.929

(1.09)

\section{SIFI * Exchange Rate Derivatives for Hedging}

N

\begin{tabular}{l}
\hline$N$ \\
Adjusted-R2 \\
\hline Panel C: Credit Risk Beta \\
Market Liquidity
\end{tabular}

Funding Liquidity

Non-Performing Loans

Loan Charge-Offs

Loan Loss Provisions

Size

Capital Ratio

$11803 \quad 11802$

$-0.503 * * *-0.507 * * *$

$-0.503 *$

$(-9.23)$

$-0.393 * * *$

$-4.694 * * *$

$(-9.31)$

$-0.397 * * *$

$(-3.01)$

$-4.679 * * *$

$(-11.26)$
$-5.713 * *$

$(-11.35)$

$-5.644 *$

$(-2.56)$

$6.249 * * *$

$6.299 * * *$

(3.07)

(3.07)

$0.0501 * * *$

$0.0444 * *$

(2.86)

$(2.41)$

-0.00527
$(-0.54)$

(2.4)

$-0.393 * * *$

11803

11803

(-6.37)

$-0.511 * * *$

$(-9.37)$

$-0.389 * * *$

$(-1.01)$

$(-2.94)$

$-4.416^{* * *}$

$-4.716 * * *$

$(-11.39)$

$-3.515$

$-5.920 * * *$

$(-2.68)$

$4.552 *$

$6.485 * * *$

(3.19)

$(1.88)$

$0.0495 * * *$

$(5.38)$

(2.83)

$(5.38)$

$-0.00646$

$(-1.25)$

$(-0.69)$

\begin{tabular}{llll}
11803 & 11803 & 11802 & 11803 \\
0.165 & 0.167 & 0.167 & 0.166 \\
\hline
\end{tabular}

$-0.512 * * * \quad-0.503 * * *$

$-0.505 * * *$

$(-9.39)$

$(-9.23)$

$(-9.28)$

$-0.507 * * *$

$0.511 * * *$

$-0.389 * * *$

$-0.412 * * *$

$-0.411 * * *$

$(-9.29)$

$(-9.38)$

$(-2.94) \quad(-3.13)$

$(-3.11)$

$(-2.90)$

$(-2.94)$

(-11.38)

$-4.689 * * *$

$-4.659 * * *$

$-4.706 * * *$

$-4.709 * * *$

$-5.475 * *$

$(-11.38)$

$(-11.37)$

$(-2.68)$

$(-2.46)$

$(-2.65)$

$(-2.68)$

$6.490 * * *$

$6.203 * * *$

$6.011 * * *$

$6.388 * *$

$(-2.68)$

(3.19)

$(3.05)$
$0.0476 * *$

(2.93)

(3.13)

(3.18)
(2.83)
$(2.71)$

(2.47)

$0.0517 * *$

$.0496 * * *$

$-0.00646-0.00483$

$(-0.49)$

(2.94)

(2.83)

(-0.69)

(-0.60)

(-0.68) 
Table 1.9. Determinants of Interest Rate, Exchange Rate, and Credit Risk Betas, and Interaction Terms

Tier1 Ratio

GDP Growth

Credit Derivatives

Capital Ratio * Credit Derivatives

Tier1 Ratio * Credit Derivatives

Size * Credit Derivatives

SIFI $*$ Credit Derivatives

Gross Credit Protection

Net Credit Protection Bought

Capital Ratio * Gross Credit Protection

Capital Ratio * Net Credit Protection Bought

Tier1 Ratio * Gross Credit Protection

Tier1 Ratio *Net Credit Protection Bought

Size * Gross Credit Protection

Size * Net Credit Protection Bought

SIFI * Gross Credit Protection

SIFI * Net Credit Protection Bought

\begin{tabular}{|c|c|c|c|c|c|c|c|c|}
$\mathbf{1}$ & $\mathbf{2}$ & $\mathbf{3}$ & $\mathbf{4}$ & $\mathbf{5}$ & $\mathbf{6}$ & $\mathbf{7}$ & $\mathbf{8}$ & $\mathbf{9}$ \\
& -0.211 & & & & -0.196 \\
& $(-1.36)$ & & & & & $(-1.28)$ \\
$0.00615^{* * *}$ & $0.00628^{* * *}$ & $0.00453^{* *}$ & $0.00617^{* * *}$ & $0.00614^{* * *}$ & $0.00601 * * *$ & $0.00617^{* * *}$ & $0.00617^{* * *}$ & $0.00615^{* * *}$ \\
$(3.39)$ & $(3.46)$ & $(2.36)$ & $(3.40)$ & $(3.38)$ & $(3.32)$ & $(3.40)$ & $(3.40)$ & $(3.39)$
\end{tabular}

$\begin{array}{llll}0.511 * * * & 0.371 * * * & 0.0599 * * * & 0.0702\end{array}$

(6.12) (4.60) (3.83)

$-3.116^{* * * *}$

$(-5.01)$

\section{$-4.753 * * *$}

$(-3.73)$

$0.0329 * * *$

(10.66)

0.00645

(0.15)

$\begin{array}{ccccc}0.0704 * * * & 0.333 * * * & 0.168 * & 1.540 * & 0.0739 \\ (4.26) & (3.72) & (1.69) & (1.76) & (1.42) \\ 0.337 * * & 11.09 * * * & 11.52 * * * & -16.35 * * * & -0.133\end{array}$

(2.12)

$1.52 * * *$

$(-2.64)$

$-0.133$

$-1.655^{* *}$

$(-2.41)$

$-89.72 * * *$

$(-4.12)$

$-1.563$

$(-1.00)$

$-185.7 * * *$

$(-3.96)$

$-0.0693 *$

$(-1.69)$

$0.791 * * *$

(2.71)

$-0.00292$

$(-0.05)$

0.486

(0.40)

$N$

Adjusted-R2

10984

10983

9720

10984

10984

0.163

0.163

10984

0.165

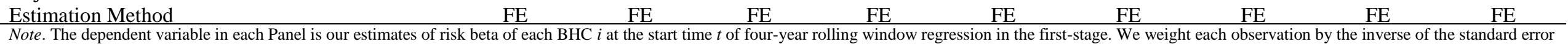

10983

0.165

0.163

0.163

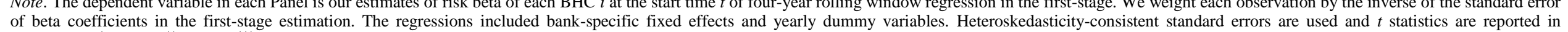
parentheses. ${ }^{*} p<0.10,{ }^{* * *} p<0.05,{ }^{* * *} p<0.01$

Source: Financial data is from FR Y-9C; Risk betas are computed from the four-factor model using data from Center for Research in Security Prices (CRSP) database and Federal Reserve monthly Statistical Releases. 


\subsubsection{The global financial crisis and financial derivatives}

We now analyze the impact of the global financial crisis on the relationship between financial derivatives and systematic risk exposures. Table 1.10 shows that the positive relationship between financial derivatives and systematic risk exposures remains unchanged when we include the crisis dummy Crisis. Crisis has a negative (but largely insignificant) impact on systematic interest rate risk, a positive and insignificant impact on systematic exchange rate risk, and a positive and highly significant impact on systematic credit risk. During the global financial crisis, the positive relationship between Interest Rate Derivatives and systematic interest rate risk intensifies, whereas the positive relation between Credit Derivatives and systematic credit risk becomes less pronounced. Crisis increases the positive relationship between financial derivatives for hedging and systematic interest rate risk, whereas it decreases the positive relationship between Gross Credit Protection and systematic credit risk. This provides some (but limited) evidence that during the crisis, BHCs strove to classify more financial derivatives as derivatives for hedging purposes, which strengthens the positive relationship between derivatives for hedging and systematic risks.

Table 1.10. Impact of Financial Crisis on the Determinants of Interest Rate, Exchange Rate, and Credit Risk

\begin{tabular}{|c|c|c|c|c|}
\hline \multicolumn{5}{|c|}{ Betas } \\
\hline Variable & 1 & 2 & 3 & 4 \\
\hline \multicolumn{5}{|c|}{ Panel A: Interest Rate Risk Beta } \\
\hline \multirow[t]{2}{*}{ Interest Margin } & $4.660 * * *$ & $4.658^{* * *}$ & $4.526 * * *$ & $4.449 * * *$ \\
\hline & $(6.04)$ & $(6.03)$ & $(5.87)$ & $(5.81)$ \\
\hline \multirow{2}{*}{ C\&I Loans } & -0.380 & -0.393 & -0.329 & -0.420 \\
\hline & $(-0.98)$ & $(-1.01)$ & $(-0.85)$ & $(-1.09)$ \\
\hline \multirow[t]{2}{*}{ Mortgage Loans } & 0.130 & 0.142 & 0.163 & 0.334 \\
\hline & $(0.55)$ & $(0.60)$ & $(0.69)$ & $(1.42)$ \\
\hline Other Loans & $\begin{array}{c}-1.546 * * * \\
(-3.21)\end{array}$ & $\begin{array}{c}-1.543 * * * \\
(-3.20)\end{array}$ & $\begin{array}{c}-1.461 * * * \\
(-3.04)\end{array}$ & $\begin{array}{c}-1.319 * * * \\
(-2.80)\end{array}$ \\
\hline \multirow[t]{2}{*}{ Domestic Deposits } & $-0.869 * * *$ & $-0.887^{* * * *}$ & $-0.755 * * *$ & $-0.745^{* * *}$ \\
\hline & $(-3.36)$ & $(-3.43)$ & $(-2.93)$ & $(-2.90)$ \\
\hline GAP Ratio & $\begin{array}{c}-0.00694 \\
(-1.57)\end{array}$ & $\begin{array}{c}-0.00699 \\
(-1.58)\end{array}$ & $\begin{array}{c}-0.00605 \\
(-1.37)\end{array}$ & $\begin{array}{c}-0.00466 \\
(-1.05)\end{array}$ \\
\hline Size & $0.641 * * *$ & $0.631 * * *$ & $0.665 * * *$ & $0.636^{* * *}$ \\
\hline Capital Ratio & $\begin{array}{c}10.41) \\
0.0954 \\
(0.86)\end{array}$ & $\begin{array}{c}(10.28) \\
0.0934 \\
(0.86)\end{array}$ & $\begin{array}{c}(10.90) \\
0.100 \\
(0.87)\end{array}$ & $\begin{array}{l}(10 . / 8) \\
0.101 \\
(0.94)\end{array}$ \\
\hline GDP Growth & $\begin{array}{c}-0.00624 \\
(-1.28)\end{array}$ & $\begin{array}{c}-0.00599 \\
(-1.23)\end{array}$ & $\begin{array}{c}-0.00651 \\
(-1.34)\end{array}$ & $\begin{array}{c}-0.00510 \\
(-1.05)\end{array}$ \\
\hline Interest Rate Derivatives & $\begin{array}{c}0.116 * * * \\
(4.50)\end{array}$ & $\begin{array}{c}0.103 * * * \\
(4.00)\end{array}$ & & \\
\hline Crisis & $\begin{array}{c}-0.0218 \\
(-0.74)\end{array}$ & $\begin{array}{c}-0.0279 \\
(-0.95)\end{array}$ & $\begin{array}{c}-0.0172 \\
(-0.59)\end{array}$ & $\begin{array}{c}-0.118 * * * \\
(-3.56)\end{array}$ \\
\hline \multicolumn{2}{|c|}{ Crisis * Interest Rate Derivatives } & $\begin{array}{c}0.0191 * \\
(1.65)\end{array}$ & & \\
\hline \multicolumn{2}{|c|}{ Interest Rate Derivatives for Trading } & & $\begin{array}{c}0.101 * * * \\
(4.15)\end{array}$ & $\begin{array}{c}0.0827 * * * \\
(3.48)\end{array}$ \\
\hline \multicolumn{2}{|c|}{ Interest Rate Derivatives for Hedging } & & $\begin{array}{c}0.474 * * * \\
(4.87)\end{array}$ & $\begin{array}{c}0.463 * * * \\
(4.98)\end{array}$ \\
\hline \multicolumn{2}{|c|}{ Crisis * Interest Rate Derivatives for Trading } & & & $\begin{array}{l}0.0146 \\
(1.50)\end{array}$ \\
\hline \multicolumn{2}{|c|}{ Crisis * Interest Rate Derivatives for Hedging } & & & $\begin{array}{c}3.029 * * * \\
(5.38) \\
\end{array}$ \\
\hline $\begin{array}{l}N \\
\text { Adjusted-R2 }\end{array}$ & $\begin{array}{l}11795 \\
0.199 \\
\end{array}$ & $\begin{array}{l}11795 \\
0.199 \\
\end{array}$ & $\begin{array}{l}11799 \\
0.200 \\
\end{array}$ & $\begin{array}{l}11799 \\
0.209 \\
\end{array}$ \\
\hline
\end{tabular}

Panel B: Exchange Rate Risk Beta 
Table 1.10. Impact of Financial Crisis on the Determinants of Interest Rate, Exchange Rate, and Credit Risk

Variable

Assets in Foreign Currencies

Crisis * Exchange Rate Derivatives

$(1.06)$

Exchange Rate Derivatives for Trading

$0.500 * * * \quad 0.467 * * *$

Exchange Rate Derivatives for Hedging

Crisis * Exchange Rate Derivatives for Trading

$(4.75)$

0.0581

$(1.48)$

Crisis * Exchange Rate Derivatives for Hedging

$-2.288$

$(-1.26)$

\begin{tabular}{|c|c|c|c|c|}
\hline $\begin{array}{l}N \\
\text { Adjusted-R2 }\end{array}$ & $\begin{array}{l}11803 \\
0.165 \\
\end{array}$ & $\begin{array}{l}11803 \\
0.165 \\
\end{array}$ & $\begin{array}{l}11803 \\
0.165 \\
\end{array}$ & $\begin{array}{l}11803 \\
0.165 \\
\end{array}$ \\
\hline \multicolumn{5}{|l|}{ Panel C: Credit Risk Beta } \\
\hline Market Liquidity & $\begin{array}{c}-0.506 * * * \\
(-9.29)\end{array}$ & $\begin{array}{c}-0.506 * * * \\
(-9.29)\end{array}$ & $\begin{array}{c}-0.507 * * * \\
(-9.30)\end{array}$ & $\begin{array}{c}-0.507 * * * \\
(-9.31)\end{array}$ \\
\hline \multirow[t]{2}{*}{ Funding Liquidity } & $-0.367 * * *$ & $-0.373 * * *$ & $-0.367 * * *$ & $-0.375 * * *$ \\
\hline & $(-2.77)$ & $(-2.82)$ & $(-2.77)$ & $(-2.83)$ \\
\hline \multirow[t]{2}{*}{ Non-Performing Loans } & $-4.747 * * *$ & $-4.747 * * *$ & $-4.742 * * *$ & $-4.741 * * *$ \\
\hline & $(-11.45)$ & $(-11.45)$ & $(-11.44)$ & $(-11.43)$ \\
\hline \multirow{2}{*}{ Loan Charge-Offs } & $-5.977 * * *$ & $-5.999 * * *$ & $-5.972 * * *$ & $-6.003 * * *$ \\
\hline & $(-2.71)$ & $(-2.72)$ & $(-2.71)$ & $(-2.72)$ \\
\hline Loan Loss Provisions & $\begin{array}{c}6.401 * * * \\
(3.15)\end{array}$ & $\begin{array}{c}6.438 * * * \\
(3.16)\end{array}$ & $\begin{array}{c}6.403 * * * \\
(3.15)\end{array}$ & $\begin{array}{c}6.451 * * * \\
(3.17)\end{array}$ \\
\hline Size & $\begin{array}{c}0.0482 * * * \\
(2.75)\end{array}$ & $\begin{array}{c}0.0482 * * * \\
(2.75)\end{array}$ & $\begin{array}{c}0.0481 * * * \\
(2.75)\end{array}$ & $\begin{array}{c}0.0481 * * * \\
(2.75)\end{array}$ \\
\hline Capital Ratio & $\begin{array}{c}-0.00750 \\
(-0.79)\end{array}$ & $\begin{array}{c}-0.00732 \\
(-0.76)\end{array}$ & $\begin{array}{c}-0.00750 \\
(-0.79)\end{array}$ & $\begin{array}{c}-0.00732 \\
(-0.76)\end{array}$ \\
\hline GDP Growth & $\begin{array}{c}0.00615 * * * \\
(3.39)\end{array}$ & $\begin{array}{c}0.00607 * * * \\
(3.35)\end{array}$ & $\begin{array}{c}0.00612 * * * \\
(3.37)\end{array}$ & $\begin{array}{c}0.00601 * * * \\
(3.32)\end{array}$ \\
\hline Credit Derivatives & $\begin{array}{c}0.0756 * * * \\
(4.70)\end{array}$ & $\begin{array}{c}0.121 * * * \\
(6.08)\end{array}$ & & \\
\hline Crisis & $\begin{array}{c}0.0353^{* * * *} \\
(3.17)\end{array}$ & $\begin{array}{c}0.0364 * * * \\
(3.27)\end{array}$ & $\begin{array}{c}0.0353 * * * \\
(3.18)\end{array}$ & $\begin{array}{c}0.0371 * * * \\
(3.33)\end{array}$ \\
\hline \multicolumn{2}{|l|}{ Crisis $*$ Credit Derivatives } & $\begin{array}{c}-0.0499 * * * \\
(-3.83)\end{array}$ & & \\
\hline \multicolumn{2}{|l|}{ Gross Credit Protection } & & $\begin{array}{c}0.0699 * * * \\
(4.22)\end{array}$ & $\begin{array}{c}0.117 * * * \\
(5.90)\end{array}$ \\
\hline \multicolumn{2}{|l|}{ Net Credit Protection Bought } & & $\begin{array}{c}0.338^{* *} \\
(2.12)\end{array}$ & $\begin{array}{c}0.584 * * \\
(2.37)\end{array}$ \\
\hline \multicolumn{2}{|l|}{ Crisis $*$ Gross Credit Protection } & & & $\begin{array}{c}-0.0482 * * * \\
(-3.67)\end{array}$ \\
\hline Crisis * Net Credit Protection Bought & & & & $\begin{array}{l}-0.392 \\
(-1.18) \\
\end{array}$ \\
\hline $\begin{array}{l}N \\
\text { Adjusted-R2 }\end{array}$ & $\begin{array}{l}10984 \\
0.163\end{array}$ & $\begin{array}{l}10984 \\
0.164\end{array}$ & $\begin{array}{l}10984 \\
0.163\end{array}$ & $\begin{array}{l}10984 \\
0.164\end{array}$ \\
\hline Estimation Method & $\mathrm{FE}$ & FE & FE & FE \\
\hline
\end{tabular}

Prices (CRSP) database and Federal Reserve monthlv Statistical Releases. 


\subsubsection{The impact of financial derivatives on market risk and idiosyncratic risk}

We now test the impact of financial derivatives on idiosyncratic risk (Table 1.11) and market risk (Table 1.12). Given the bounded nature of idiosyncratic risk $\left(1-\mathrm{R}^{2}\right)$, we use its logistic transformation risk (i.e., $\log \left(\frac{1-\mathrm{R}^{2}}{\mathrm{R}^{2}}\right)$ ) as the dependent variable in Table 1.11. Table 1.11 shows that larger and well-capitalized BHCs face higher idiosyncratic risk than smaller and weakly-capitalized BHCs. The relationship between total financial derivatives and idiosyncratic risk is negative and significant (especially for financial derivatives for trading). The negative relationship exists between exchange rate derivatives and idiosyncratic risk. Interest rate derivatives are negatively related to idiosyncratic risk for total BHCs and for large BHCs, but positively for small BHCs. Credit derivatives are positively associated with idiosyncratic risk for total BHCs, but negatively for small BHCs.

Table 1.12 indicates that larger BHCs and BHCs with higher capital ratios are exposed to higher market risk. The relationship between financial derivatives and market risk varies across types of financial derivatives: Exchange Rate Derivatives are negatively and significantly related to market risk, whereas Interest Rate Derivatives are positively related to market risk. ${ }^{24}$

\footnotetext{
${ }^{24}$ To assess the reliability of our results, we conducted several robustness checks. We used the change in the difference between BBB bond yield and the risk-free rate in the first-stage regression as an alternative definition of Credit Risk. In addition, we used several different instrument variables in our estimations. Our findings are qualitatively robust compared to alternative specifications. The robustness checks results can be found in Appendix A from Table A.2 to Table A.10.
} 
Variable

Idiosyncratic Risk

Size

Capital Ratio

GDP Growth

Interest Rate Derivatives

Exchange Rate Derivatives

Credit Derivatives

Total Financial Derivatives

Financial Derivatives for Trading

Financial Derivatives for Hedging

$N$

Adjusted-R2

Hansen J Statistic ( $p$-value)

Number of Instruments

Estimation Method

Table 1.11. The Impact of Financial Derivatives on Scaled Idiosyncratic Risk $\left(1-R^{2}\right)$ Total BHCs Large BHCs

\begin{tabular}{|c|c|c|c|c|c|c|c|c|c|}
\hline $\begin{array}{c}0.227 * * * \\
(6.51)\end{array}$ & $\begin{array}{c}0.423 * * * \\
(8.29)\end{array}$ & $\begin{array}{c}0.232 * * * \\
(6.63)\end{array}$ & $\begin{array}{c}0.232 * * * \\
(6.63)\end{array}$ & $\begin{array}{c}0.426 * * * \\
(4.43)\end{array}$ & $\begin{array}{c}0.488 * * * \\
(4.91)\end{array}$ & $\begin{array}{c}0.470 * * * \\
(5.18)\end{array}$ & $\begin{array}{c}0.202 * * * \\
(5.51)\end{array}$ & $\begin{array}{c}0.428 * * * \\
(7.29)\end{array}$ & $\begin{array}{c}0.208 * * * \\
(5.63)\end{array}$ \\
\hline $\begin{array}{c}-0.0579 * * \\
(-2.44)\end{array}$ & $\begin{array}{c}0.518 * * \\
(2.15)\end{array}$ & $\begin{array}{c}-0.0576 * * \\
(-2.43)\end{array}$ & $\begin{array}{c}-0.0575 * * \\
(-2.42)\end{array}$ & $\begin{array}{c}2.797 * * \\
(2.33)\end{array}$ & $\begin{array}{l}2.289 \\
(1.62)\end{array}$ & $\begin{array}{c}4.040 * * * \\
(3.20)\end{array}$ & $\begin{array}{c}-0.0678 * * \\
(-2.42)\end{array}$ & $\begin{array}{c}0.513 * * \\
(1.98)\end{array}$ & $\begin{array}{c}-0.0722 * * \\
(-2.36)\end{array}$ \\
\hline 0.000744 & $0.0143 * *$ & 0.000905 & 0.000911 & $0.0356 * *$ & $0.0245^{*}$ & $0.0379 * * *$ & -0.00337 & 0.00681 & -0.00307 \\
\hline$(0.19)$ & $(2.55)$ & $(0.24)$ & $(0.24)$ & $(2.45)$ & $(1.76)$ & $(2.61)$ & $(-0.85)$ & $(1.14)$ & $(-0.77)$ \\
\hline$-0.0363 * * *$ & $-0.0358 * * *$ & & & $-0.0302 * * *$ & $-0.0317 * * *$ & & $0.0483 *$ & $0.0659 *$ & \\
\hline$(-4.43)$ & $(-3.81)$ & & & $(-4.50)$ & $(-4.21)$ & & (1.94) & (1.92) & \\
\hline$-0.368 * * *$ & $-0.441 * * *$ & & & $-0.316 * * *$ & $-0.375 * * *$ & & $-1.027 * * *$ & $-0.975 * * *$ & \\
\hline$(-6.33)$ & $(-7.07)$ & & & $(-6.72)$ & $(-6.74)$ & & $(-4.71)$ & $(-4.69)$ & \\
\hline \multirow{7}{*}{$\begin{array}{c}0.0857^{*} \\
(1.82)\end{array}$} & 0.0265 & & & -0.00177 & 0.00331 & & $-0.319 * *$ & $-0.615 * *$ & \\
\hline & $(0.52)$ & & & $(-0.04)$ & $(0.07)$ & & $(-2.22)$ & $(-2.33)$ & \\
\hline & & $\begin{array}{c}-0.0393 * * * \\
(-5.22)\end{array}$ & & & & & & & \\
\hline & & & $-0.0394 * * *$ & & & $-0.0367 * * *$ & & & $-0.0519 * *$ \\
\hline & & & $(-5.18)$ & & & $(-5.86)$ & & & $(-2.39)$ \\
\hline & & & -0.0312 & & & $-0.223 * * *$ & & & 0.00721 \\
\hline & & & $(-0.73)$ & & & $(-2.91)$ & & & $(0.15)$ \\
\hline 11888 & 4374 & 11888 & 11888 & 757 & 709 & 757 & 11131 & 3670 & 11131 \\
\hline \multirow[t]{2}{*}{0.281} & 0.421 & 0.279 & 0.279 & 0.591 & 0.616 & 0.577 & 0.269 & 0.401 & 0.266 \\
\hline & $2.40(0.301)$ & & & & $4.98(0.09)$ & & & $3.83(0.147)$ & \\
\hline
\end{tabular}

FE $\quad 18 \quad 18$

IV

18 estimation. The regressions included bank-specific fixed effects and yearly dummy variables. Heteroskedasticity-consistent standard errors are used and $t$ statistics are reported in parentheses. ${ }^{*} p<0.10,{ }^{* * * *} p<0.05$, $p<0.01$.

Source: Financial data is from FR Y-9C; Risk betas are computed based on the four-factor model using data from Center for Research in Security Prices (CRSP) database and Federal Reserve monthly Statistical Releases. 
Table 1.12. The Impact of Financial Derivatives on Market Risk Beta

Variable

\section{Market Risk Beta}

Size

Capital Ratio

GDP Growth

Interest Rate Derivatives

Exchange Rate Derivatives

Credit Derivatives

$N$

Adjusted-R2

Hansen J Statistic ( $p$-value)

Number of Instruments

Estimation Method
Total Sample

Large BHCs

Small BHCs

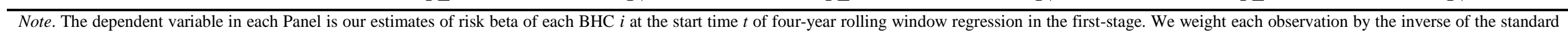

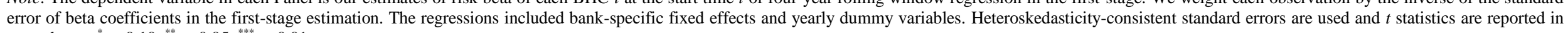
parentheses. ${ }^{*} p<0.10,{ }^{* *} p<0.05,{ }^{* * *} p<0.01$.

Source: Financial data is from FR Y-9C; Risk betas are computed based on the four-factor model using data from Center for Research in Security Prices (CRSP) database and Federal Reserve monthly Statistical Releases. 


\subsection{Main Findings}

We examine whether financial derivatives magnify or mitigate systematic interest rate risk, exchange rate risk, and credit risk of publicly traded U.S. BHCs from 1997 to 2012. In the first-stage regression, we obtain betas that measure systematic interest rate risk, exchange rate risk, and credit risk. In the second stage, we regress risk betas generated in the first stage against financial derivatives variables.

We show that financial derivatives are positively and significantly related to systematic risk exposures of BHCs. Higher use of interest rate derivatives, exchange rate derivatives, and credit derivatives corresponds to greater systematic interest rate risk, exchange rate risk, and credit risk. The positive relationship strengthens with the size of a BHC. We establish a positive relationship between trading derivatives and risks as well as between hedging derivatives and risks.

Policy implications immediately follow. Our analysis shows that further caution is needed regarding BHCs' engagement in the derivatives business, giving further support for limiting the use of financial derivatives across BHCs. Many recent regulatory attempts aim to separate commercial banking from more risky banking activities, such as engagement in proprietary trading (see the Volker rule under the Dodd-Frank Wall Street Reform and Consumer Protection Act and Independent Commission on Banking, 2011; Boot and Ratnovksi, 2013 for theoretical analysis). Regulators need to think how to reverse the positive relationship between derivatives and systematic risks and at the same time preserve the efficiency of bank risk management (see also Thakor (2012b)). In this light, regulators aim to separate financial derivatives that are used for hedging from the ones generated in the proprietary trading business. The problem that may occur is that it is difficult to determine when financial derivatives are used for trading purposes and when for hedging purposes. We show that financial derivatives for hedging (and trading) purposes are associated with higher systematic risks of BHCs. This indicates that prohibiting financial derivatives for trading may give a false sense of safety because risks may then concentrate in financial derivatives for hedging purposes. 


\section{QUALITY OF BANK CAPITAL AND BANK LENDING BEHAVIOR DURING THE GLOBAL FINANCIAL CRISIS ${ }^{25}$}

\subsection{Overview}

Using a worldwide bank sample from 2000 to 2010, this chapter analyzes the determinants of bank lending behavior during the global financial crisis, highlighting the role of bank capital. It reveals that the high quality of the bank funding strategy (tier 1 bank capital and retail deposits) and prevalent government backing were crucial to continuous bank lending during the crisis period. This effect was especially pronounced in non-OECD and BRIC countries. It also points out that, although higher use of tier 2 capital and interbank deposits could be important for increased lending during a normal period, this did not support lending activities during the financial crisis. This chapter concludes by suggesting that in crisis periods, high-quality bank capital is a bank's competitive strength.

\subsection{Introduction}

The global financial crisis of 2008-2012 was propagated through the banking systems across the world and triggered unprecedented consequences for the global economy. The regulators pushed for enhanced regulation, incorporated in the revised, Basel III capital regulatory framework (Basel Committee on Banking Supervision, 2010). As Ben Bernanke, the chairman of the Federal Reserve, put it, "this framework would require banking organizations to hold more and higher quality capital . . . improving the resilience of the U.S. banking system in times of stress, thus contributing to the overall health of the U.S. economy." ${ }^{26}$ In Europe, Andrea Enria, the chairman of the European Banking Authority, hailed improved capital positions of European banks by noting that "European banks are now in a stronger position, which should support lending to the real economy . . ."27 In contrast, bankers strongly objected to this reasoning. Vikram Pandit, former CEO of Citigroup, argued that "double-digit ratios will undermine lending, slow capital formation, lower demand and restrict growth." 28

This chapter empirically evaluates whether bank funding structure affects bank lending and, in particular, whether the quality of bank capital matters for lending growth. In line with the Basel accords, we distinguish between high-quality bank capital - that is, tier 1 capital with the highest loss-absorbing capacity - and supplementary tier 2 bank capital with a lower loss-absorbing capacity.

We use annual financial data for banks worldwide from 2000 to 2010 to discern the relationships

\footnotetext{
${ }^{25}$ This chapter is co-authored with Marko Košak, Igor Lončarski and Matej Marinč. We wish to thank Jonathan Batten, Arnoud Boot, Nadia Massoud, and Razvan Vlahu, as well as the participants at the EBES 2012 Conference, the Australasian Finance and Banking Conference 2012, and INFINITI 2013 for valuable comments. This chapter has been published in International Review of Financial Analysis, 37, 2015, 168-183.

${ }^{26}$ Statement by Chairman Ben S. Bernanke, 7 June, 2012, http://www.federalreserve.gov/newsevents/press/bcreg/bernanke20120607a.htm

27 www.eba.europa.eu/News--Communications/Year/2012/Update-implementation-capital-exercise.aspx

28 Vikram Pandit, We must rethink Basel or growth will suffer, Financial Times, 10 November, 2010.
} 
between bank lending and bank capital in normal times and during the global financial crisis. Figure 2.1 provides the first inspection of the role of the bank capital for bank lending activity. In Figure 2.1, banks are split in quartiles according to their tier 1 capital ratio (tier 1 capital per risk-weighted assets). This shows that lending grew faster for banks with a high tier 1 capital ratio (a tier 1 capital ratio in the highest quartile) than for banks with a low tier 1 capital ratio.

Figure 2.1. Loan Growth of Different Subsamples by Tier 1

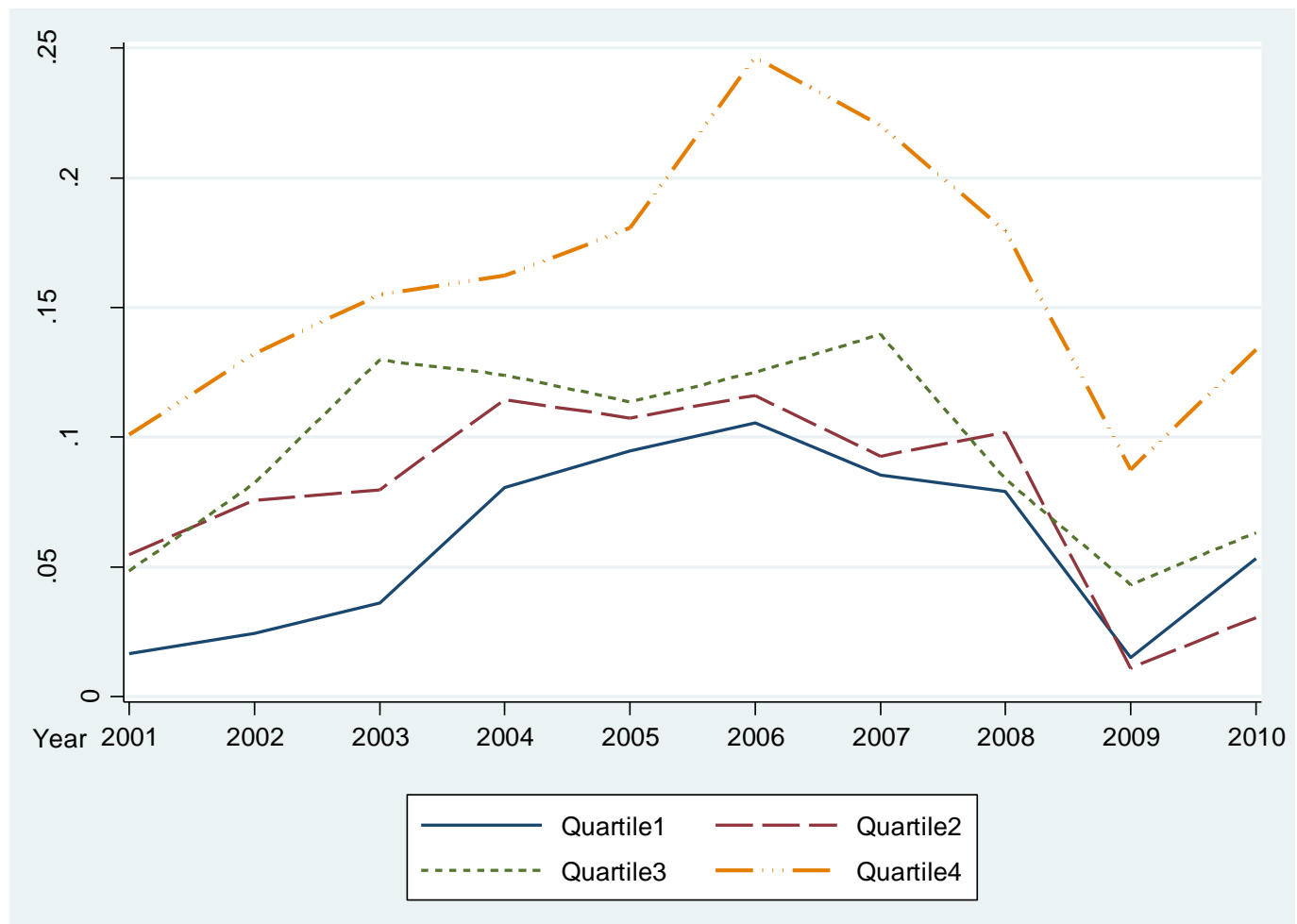

Source: BankScope, 2011.

Our findings provide support for the hypothesis that the higher quality of the bank funding side (i.e., a high tier 1 bank capital ratio, high proportion of customer deposits, and prevalent government support) better supports bank lending during crisis times. We find that the tier 1 capital ratio positively affected bank loan growth during the global financial crisis, and this relation is particularly strong within the subsample of non-OECD countries and BRIC countries. This indicates that the tier 1 capital ratio helps banks overcome periods of distress and maintain or even intensify their lending activity, especially for developing countries. In contrast, we generally do not find tier 2 capital to have been statistically significantly related to lending growth during the global financial crisis, potentially indicating that tier 2 capital does not provide adequate support for bank lending activities during a financial crisis.

Interestingly, we show that during the global financial crisis, banks cut back on lending more if competing banks had high tier 1 ratios. This indicates that high-quality capital strengthens the competitive position of a bank in a financial crisis. Our analysis confirms the findings in Berger and Bouwman (2013) from the U.S. banking sector and extends them to a global setting. In particular, Berger and Bouwman (2013) show that capital increases the survival probability and market share of banks. The effect occurs at all times for small banks and during banking crises 
for medium and large banks. Our evidence shows that small banks lent more if they had high levels of bank capital, whereas large banks lent more in the global financial crisis (but less in normal times) if their competing banks had low levels of bank capital. Hence, in a crisis, bank capital directly helps small banks, whereas large banks gain a competitive advantage against weakly capitalized competitors.

Banks' funding risks may stem not only from the insufficient levels of bank capital, but also from an inadequate structure of liabilities that banks took over in times of accelerated economic growth and the abundance of liquidity. We find some (limited) evidence that interbank deposits negatively affected bank lending during the global financial crisis. Banks tried to compensate for this by turning to more stable funding sources, such as retail deposits (European Central Bank, 2011). We find some evidence that customer (retail) deposits were sticky and acted as a stable source of funding even during the global financial crisis. In particular, we show that customer deposits were positively related to loan growth during the global financial crisis.

During the crisis, banks were largely supported by the governments to overcome refinancing difficulties. We control for various aspects of ownership and indirect government support. We find a positive impact of government ownership (and some limited evidence for a negative impact of foreign ownership) on bank loan growth during the global financial crisis. This points to the benefits of government ownership in mitigating the credit crunch. ${ }^{29}$

The remainder of this chapter is organized as follows; In Section 2.3, we look at previous studies and define the main hypotheses. In Section 2.4, we describe our data. In Section 2.5, we present the empirical model. Section 2.6 presents and discusses the results. Section 2.7 provides several robustness checks. Section 2.8 concludes this chapter.

\subsection{Previous Studies and Development of Hypotheses}

The literature on the impact of bank capital structure on bank lending was scarce prior to the global financial crisis from 2008 to 2010 and did not distinguish between tier 1 and tier 2 capital. For example, De Haas and Van Lelyveld (2010) analyze micro and macro determinants of multinational bank lending, but consider an aggregate equity-to-total-assets ratio to account for the solvency of individual banks. Gambacorta and Mistruli (2004) analyze the role of capital in bank lending behavior and find that well-capitalized banks can better shield their lending from monetary policy shocks. Lending decisions of banks in relation to their capitalization are also addressed in studies by Admati et al. (2010) and Jiménez et al. (2012), who observe that the global financial crisis negatively affected the lending activity of banks, especially those with low capital and liquidity ratios. Using a disaggregate measure, we confirm that tier 1 bank capital (but not tier 2 bank capital) and retail or customer deposits positively affected continuous

\footnotetext{
${ }^{29}$ Although several studies point to the inefficiency of government ownership and benefits of foreign ownership on bank efficiency (see, e.g., Berger, Hasan, and Zhou (2009); Bonin, Hasan, and Wachtel (2005a, 2005b); Shen and Lin (2012); Shen, Hasan, and Lin (2014)), others stress the negative impact of foreign ownership on the quality of governance (e.g., Lensink, Meesters, and Naaborg (2008)) or analyze alternative institutional forms (e.g., Columba, Gambacorta, and Mistrulli $(2009,2010)$ argue that mutual guarantee institutions may alleviate access to finance for SMEs).
} 
lending during the financial crisis.

Our analysis is closely related to the one by Gambacorta and Marques-Ibanez (2011), which also highlights the positive effect of tier 1 capital on bank lending activities during the crisis (see also Brei, Gambacorta, and von Peter (2013)). ${ }^{30}$ Whereas these studies focus on selected advanced economies, we extend some of their perspectives to include worldwide data from 131 countries because our focus shifts beyond the biggest banks, given that the overwhelming majority of European and U.S. businesses are dependent on loans from smaller banks and their subsequent relationships (see Hancock and Wilcox (1998); Berger, Hasan, and Klapper (2004)). In addition, non-listed smaller banks faced greater difficulties in finding additional funding sources on the market during the financial crisis. Therefore, the role of tier 1 for lending of all banks (and especially small ones) during the global financial crisis warrants further scrutiny. As reported earlier, our analysis confirms that tier 1 capital is of particular importance for smaller banks.

We build the analysis around five main hypotheses related to the role of 1) tier 1 capital, 2) tier 2 capital, 3) various categories of deposits, 4) a competitive environment, including tier 1 capital of competing banks, and 5) ownership in explaining bank credit dynamics. We distinguish between periods before and after the global financial crisis.

In relation to our first key hypothesis, we investigate whether and how different types of bank capital affected bank lending in normal times and during the global financial crisis. The first role of bank capital is to serve as a buffer to absorb banks' losses and insulate banks from insolvency. The purpose of holding additional capital for banks above the required regulatory level is to protect banks against large losses during a cyclical downturn and reduce the risk of insolvency (Rajan (1994); see also Ayuso, Perez, and Saurina (2004); Jokipii and Milne (2008)). In this view, banks with high levels of bank capital (and therefore with a high capital buffer) could accommodate faster loan growth and could lend more than banks with small levels of bank capital. In addition, banks with high levels of bank capital could better weather the global financial crisis and support lending than banks with low levels of bank capital.

The second role of bank capital is to act as an incentive device that can commit banks to prudent behavior by reducing the attractiveness of risk-taking. Banks are highly leveraged institutions that operate with a broad safety net (e.g., deposit insurance schemes and implicit government bailout guarantees). This exacerbates risk-taking by bank managers and shareholders, who bet on high returns knowing that losses are primarily subsumed by debt holders and taxpayers. Only a sufficiently high level of capital puts the skin of the bankers and shareholders into the game and induces prudent lending behavior (see VanHoose (2007); Goodhart (2013)). ${ }^{31}$

\footnotetext{
30 Berrospide and Edge (2010) analyze lending by the U.S. Bank Holding Companies to confirm a positive but small effect of bank capital on lending. Cornett et al. (2011) analyze the relationship between credit supply and liquidity and capital positions of all U.S. commercial banks during the global financial crisis. They focus on liquidity risk management and do not distinguish between different types of bank capital. Carlson, Shan, and Warusawitharana (2013) develop a novel empirical matching strategy to confirm the positive relationship between capital ratios and bank lending in the U.S. during the global financial crisis.

31 Demsetz, Saidenberg, and Strahan (1996) analyze the relationship between franchise value and risk-taking in banking. In line with Keeley (1990), they show that banks with high franchise values have much to lose in insolvency. Consequently, the high-franchise-value banks hold more capital and take on less risk than banks with lower franchise value in order to prevent insolvency from occurring. Banks' risk-taking may be driven by the banks'
} 
If the bank capital acts as an incentive device, we can make the following prediction regarding bank capital and lending behavior: to the extent that excessive lending growth is a sign of risky lending behavior (see Dell'Ariccia and Marquez (2006); Foos, Norden, and Weber (2010)), well-capitalized banks will engage in more prudent behavior and therefore will expand their lending less than weakly capitalized banks.

The abundant literature on bank capital may have underestimated the importance of the quality of bank capital. This analysis focuses on the importance of the quality of bank capital for bank lending behavior. Our main hypothesis stresses that, all else being equal, banks with larger tier 1 capital ratios better overcame the global financial crisis and cut back on lending less than banks with smaller tier 1 capital ratios. In normal times, the positive effect of the tier 1 capital ratio on credit growth persists but is less pronounced. Hypothesis 2.1, if confirmed, would suggest that tier 1 capital acts as a buffer and not as an incentive device.

Hypothesis 2.1: Tier 1 capital positively affects credit growth. This effect was more pronounced during the global financial crisis.

Only a few articles analyze the difference between tier 1 bank capital and tier 2 bank capital. Demirgüç-Kunt, Detragiache, and Merrouche (2013), for example, show that the positive association between stock returns and capital is significantly stronger for higher-quality (tier 1) bank capital than it is for lower-quality (tier 2) bank capital. Barrell et al. (2011) show that an increase in the overall capital adequacy ratio reduces the risk appetite of banks, and that the proportional increase of tier 2 bank capital, within a given capital adequacy structure, increases the risk appetite of banks (see also Ashcraft (2008b)). In addition, regulators have already acknowledged the need to readjust and recalibrate their regulatory measures. ${ }^{32}$ The intention of the Basel III Accord is to significantly increase the role of tier 1 bank capital relative to tier 2 bank capital (Basel Committee on Banking Supervision, 2010). Similarly, the European Banking Authority issued a call for recapitalization of systemically important banks in the European Union by raising the core tier 1 capital ratio to $9 \%$ in 2012. Bank capital regulatory measures are very likely to affect the credit activity of banks.

The ability of a bank to raise tier 2 capital positively affects loan growth in normal times. In normal times, banks may fund their high growth strategies by relying on subordinated debt, which as a part of tier 2 capital contributes to higher regulatory capital and helps banks meet capital requirements. During the global financial crisis, the situation reversed. The main concern of a bank and its creditors became the bank's stability. A bank with a lot of subordinated debt

\footnotetext{
business models (Altunbas, Manganelli, and Marques-Ibanez (2011)) or by the macroeconomic environment (e.g., an extended period of low interest rates; see Altunbas, Gambacorta, and Marques-Ibanez (2012), or market power; see Berger, Klapper, and Turk-Ariss (2009)) and may be mitigated by recapitalization measures or regulatory interventions (Berger et al. (2012)). Rather than on risk-taking in general, our focus is on the determinants of bank lending behavior.

${ }^{32}$ Hasan, Siddique, and Sun (2015) discuss how to construct market-based capital requirements by using market data in conjunction with regulatory data to estimate a bank's total risk. They show that capital adequacy metrics thus constructed outperform VaR-based capital models as well as purely market-based capital models that rely on CDS premia.
} 
may have a hard time renewing it. The bank may need to replace subordinated debt, which may have a detrimental effect on bank liquidity and its lending capacity. Consequently, a bank with high tier 2 capital may be forced to cut back on lending when the crisis hits. Hypothesis 2.2, if confirmed, would suggest that tier 2 capital acts as a buffer during normal times, but acted as an incentive device during the global financial crisis.

Hypothesis 2.2: Tier 2 capital positively affects loan growth during normal times. During the global financial crisis, tier 2 capital negatively affected loan growth.

The third main hypothesis focuses on the impact of various types of deposits (i.e., customer deposits and interbank deposits) on credit growth in normal times and in times of crisis. On the one hand, uninsured interbank deposits may serve as the main disciplining device for bank managers not to take excessive risks - depositors would run and withdraw their funds from the bank as soon as they anticipated bank instability (Calomiris and Kahn (1991)). According to this view, banks with a large proportion of deposits would have realized bigger withdrawals of deposits during the global financial crisis and would have needed to respond with a larger decline in their credit growth.

On the other hand, core (retail customer) deposits serve as the most stable funding source for banks (Berlin and Mester (1999); Song and Thakor (2007)). The main explanation for this is that deposits are insured and that banks offer several other services and products to small depositors that effectively bind them in a long-term relationship with the bank. According to this view, banks with a large proportion of core deposits easily weathered the global financial crisis and needed to respond with a smaller decline in credit growth. ${ }^{33}$

Hypothesis 2.3: The decline in bank lending during the global financial crisis was higher for banks with higher levels of interbank deposits and lower levels of customer deposits.

The fourth hypothesis relates to the role of the competitive environment for bank lending behavior. We specifically address the issue of market concentration by including the HerfindahlHirschman index (HHI). We anticipate that higher concentration may inhibit credit growth in normal times. During the global financial crisis, however, higher concentration may (in line with Beck, Demirgüç-Kunt, and Levine (2006)) have made the banking system more resilient, which may have mitigated the credit crunch.

Hypothesis 2.4a: Higher market concentration (HHI) is associated with lower lending during normal times, but was associated with higher lending during the global financial crisis.

In addition, we investigate how a bank's lending behavior is affected by the tier 1 capital ratios of the competing banks in the specific market. Whereas bank capital may be seen as a costly source of funding for banks (Hellmann, Murdock, and Stiglitz (2000); Berger (2006); Berger and

\footnotetext{
33 Ivashina and Scharfstein (2010) provide some evidence that banks with better access to deposits restrict their lending to a lesser extent and are less affected by the banking crisis than banks with limited access to deposits.
} 
Bonaccorsi di Patti (2006)), others stress the positive role of bank capital, especially during a financial crisis (Admati, et al. (2010); Berger and Bouwman (2013)). Mehran and Thakor (2011) predict and empirically confirm that bank values are positively correlated with bank equity capital in a cross-section. Allen, Carletti, and Marquez (2011) argue that banks may choose higher capital than required by the regulators in order to commit to high monitoring of their borrowers and, by doing this, gain a competitive advantage. We construct a proxy variable, which reflects the weighted average (by assets) of tier 1 ratios of all the competitors in the market. We anticipate that positive aspects of bank capital prevail in a financial crisis, whereas the negative aspects dominate in normal times.

Hypothesis 2.4b: Higher tier 1 capital ratios of competing banks are positively associated with bank lending during normal times, but were negatively associated during the global financial crisis.

The fifth hypothesis refers to the impact of government ownership on bank lending. Empirical literature offers different views regarding the question of whether foreign- and domestically-owned banks react differently to business cycles and banking crises. Government ownership may represent an important factor, because of the implicit or/and explicit government guarantee that protects state-owned banks. Therefore, we expect to detect a positive relationship between credit growth and government ownership during the global financial crisis.

Hypothesis 2.5a: Government owned banks sustained lending better during the global financial crisis than non-government owned banks.

We also analyze the effect of foreign ownership on bank lending. Globalization of banking is transforming the way shocks are transmitted internationally. Whereas global banks may be more resilient and better prepared to handle local shocks, they also facilitate transmission of international shocks (Cetorelli and Goldberg (2009)). In particular, parent-bank fragility negatively affects lending by subsidiaries. The result is that home market shocks are easily transmitted into foreign markets (Allen et al., 2012). Evidence in De Haas and Van Lelyveld (2010) and De Haas et al. (2012) show that foreign banks sharply cut their lending during the global financial crisis.

Hypothesis 2.5b: Foreign ownership was associated with weaker lending during the global financial crisis.

Similarly, we test for the effect of the subsidiary status on bank lending behavior. Kashyap and Stein (1997) argue that local banks, especially if they are stand-alone in structure, are the least able to access liquidity when market liquidity conditions tighten. This would predict that reduction in lending to firms and households during a crisis period is higher for domestic, stand-alone banks. International banks are usually able to borrow under significantly better conditions than smaller regional or local banks. Ashcraft (2008a) demonstrates that the banks that are affiliated with a multi-bank holding company are less likely to experience financial 
distress because of capital injections by the parent company. Therefore, we expect subsidiaries to be able to rely on the financial assistance of their parent banks, which minimizes their refinancing risk and makes them more robust and also able to maintain credit activity during a crisis.

Hypothesis 2.5c: The subsidiary status of banks was associated with stronger credit growth during the global financial crisis.

In our empirical analysis, we also control for several additional factors that might be important for banks' credit growth. First, we control for bank risk taking by including loan loss provisions and by a measure for the tangibility of bank assets (fixed assets). Second, we control for the size of the banks and commercial and savings bank status. The difference in credit growth for smaller and larger banks also needs to be considered. Berger and Bouwman (2009) find that bank capital supports liquidity creation in large banks, but not in small banks. Puri, Rocholl, and Steffen (2009) show that smaller and liquidity-constrained banks reject more loan applications during the financial crisis than larger and less liquidity-constrained banks. Small banks were the most vulnerable during the global financial crisis, whereas large and multinational banks were more likely to remain stable and financially sound. In addition, their access to external capital markets facilitates replacement of lost assets (Kashyap and Stein (1997)). Hau, Langfield, and Marques-Ibanez (2013) provide evidence that large banks are also more favorably assessed by credit rating agencies which intensifies the too-big-to-fail problem.

In addition, government assistance may act as a substitute for the bank capital (see Berger and Bouwman (2013)). Hence, we control for government support by using a bank's Fitch Support Rating. We expect more stable loan growth for banks with stronger external support and lower bailout probabilities. The effect of external support is also expected to have had a stronger impact during the global financial crisis.

\subsection{Data Description}

We use the annual financial data for 4,197 banks worldwide for the period from 2000 to 2010, obtained from the BankScope database, amounting to 16,838 bank-year observations. Our sample consists of commercial, savings, and co-operative banks from 131 countries. All of the data are used on an unconsolidated basis, and they are inflation adjusted and expressed in USD.

In Table 2.1, we present descriptive statistics of the variables for the total sample over the entire time period. In the first panel, we present the results for bank-specific variables. The average value of total asset is $\$ 16.1$ billion, but the size of the banks in our sample varies quite substantially. This implies that any analysis needs to account for the size effect. Gross loans amount to $\$ 8.67$ billion, or more than $50 \%$ of the total assets for the average-sized bank, whereas loan loss provision has an average value of $\$ 71.9$ million, or approximately $0.8 \%$ of the average value of gross loans. The fixed assets only account for $\$ 130$ million, which is less than $1 \%$ of the total assets of the average bank. 
Looking at the liability side of bank balance sheets, we find that customer deposits with an average value of $\$ 9.58$ billion account for roughly $60 \%$ of the total assets of the average-sized bank. Interbank deposits with an average value of $\$ 2.10$ billion account for roughly $13 \%$ of the total assets of the average-sized bank. Furthermore, an average value of $\$ 1.27$ billion of total capital accounts for $7.8 \%$ of the total assets of the average-sized bank. The average value of tier 1 capital and tier 2 capital is \$919 million and \$313 million, respectively. We have eliminated bank-year observations with negative tier 1 capital ratio or negative total assets. The average values of tier 1 capital per risk-weighted assets (TIERI) and tier 2 capital per risk-weighted assets (TIER2) are $16.3 \%$ and $1.7 \%$, respectively. The average ratios of customer deposits to total assets (TCD) and interbank deposits to total assets $(D E P)$ are $62.8 \%$ and $8.3 \%$, respectively.

In the second panel of Table 2.1, we include two macroeconomic variables (GDP growth and Interest rates) to control for the demand-side effects on loan growth. The average value for GDP growth is $2 \%$. Variable Interest rate denotes an interest rate for prime bank customers in real terms.

We use two industry structure variables to control for the competitive environment in a given country (see the third panel of Table 2.1). Bank concentration is measured by the HerfindahlHirschman index $(H H I)$, calculated as the sum of the squares of the market shares of the asset for the three largest banks in a country. The HHI has wide variation in the sample, and the sample mean is 0.045 (see Table 2.1). For each bank, we also compute the average tier 1 capital ratio of competing banks within the same country, weighted by assets of these banks, and we denote it by COMPTIER1. The average value of COMPTIER 1 is $9.4 \%$.

To control for the impact of institutional factors on bank behavior (see, e.g., Berger and Udell (1994); Rime (2001)), we replicate the institutional and regulatory variables from the World Bank (2008) based on the methodology used in Barth, Caprio, and Levine (2004) and Demirgüç-Kunt et al. (2008); see the fourth panel of Table 2.1. The variable Overall capital stringency has a mean of 3.72. The variable Deposit insurance has a mean of 0.77.

In the fifth panel of Table 2.1, we report descriptive statistics of several dummy variables related to organizational and ownership characteristics of banks. Fifty-five percent of the banks in our sample are commercial banks, and $16.3 \%$ are savings banks. The rest are cooperative banks, real estate and mortgage banks, and specialized government credit institutions. In our sample, 9.4\% of the banks are government-owned, and 3\% are foreign-owned. Finally, 9.4\% of banks in the sample are bank subsidiaries.

In the sixth panel of Table 2.1, we present Bailout probability, as defined in Gropp, Hakenes, and Schnabel (2010). Bailout probability measures the probability that a bank, upon having financial difficulties, is supported by the government. Bailout probability is calculated on the basis of the Fitch Support Rating variable, adjusted for potential government ownership in the bank. The average value of the variable Bailout probability is 0.68 indicating an average long-term rating above $\mathrm{BB}-$. This means on average a moderate probability of government 
support due to uncertainties regarding the ability or propensity of the government. Table B.1 in Appendix B summarizes the variables used, defines them, and describes the data sources. ${ }^{34}$

The correlations between main variables are shown in Table 2.2. We observe that size is significantly negatively correlated with credit growth and the tier 1 ratio, but significantly positively correlated with the tier 2 ratio and interbank deposits. Although statistically not significant, the correlation between size and total customer deposits is positive. The tier 1 ratio is significantly negatively correlated with the tier 2 ratio (it seems that the two act as substitutes) and total customer deposits as well.

Table 2.1. Descriptive Statistics of the Total Sample of Banks, 2000-2010

\begin{tabular}{|c|c|c|c|c|c|}
\hline Variables & Obs & Mean & Std. Dev. & $\mathrm{p} 10$ & p90 \\
\hline \multicolumn{6}{|l|}{ Bank specific variables } \\
\hline Gross loans (\$ million) & 16,838 & 8,665 & 37,153 & 65.72 & 17,681 \\
\hline Total capital (\$ million) & 15,256 & 1,270 & 8,187 & 14.96 & 1,867 \\
\hline Tier 1 capital $(\$$ million $)$ & 15,259 & 919 & 4,451 & 14.06 & 1,589 \\
\hline Tier 2 capital ( $\$$ million $)$ & 15,084 & 313 & 2,471 & 0.00 & 340 \\
\hline Total customer deposits ( $\$$ million) & 16,838 & 9,578 & 53,319 & 63.97 & 18,091 \\
\hline Interbank deposits ( $\$$ million) & 12,114 & 2,094 & 12,595 & 0.12 & 2,059 \\
\hline Total asset ( $\$$ million $)$ & 16,838 & 16,108 & 79,622 & 122 & 28,496 \\
\hline Loan loss provision(\$ million) & 16,838 & 71.89 & 474 & 0.00 & 105 \\
\hline Fixed asset ( $\$$ million) & 16,838 & 130 & 592 & 1.00 & 249 \\
\hline TAXTA & 16,620 & 2,139 & 11,301 & 0.00 & 4,387 \\
\hline$\Delta \log \mathrm{GL}$ & 16,838 & 0.103 & 0.368 & -0.073 & 0.301 \\
\hline TIER1 & 16,838 & 0.163 & 0.193 & 0.076 & 0.263 \\
\hline TIER2 & 16,838 & 0.017 & 0.039 & 0.000 & 0.042 \\
\hline TCD & 16,838 & 0.628 & 0.208 & 0.371 & 0.863 \\
\hline DEP & 12,114 & 0.083 & 0.133 & 0.000 & 0.236 \\
\hline $\mathrm{TA}$ & 16,838 & 14.231 & 2.076 & 11.713 & 17.165 \\
\hline LLP & 16,838 & 0.009 & 0.038 & 0.000 & 0.021 \\
\hline FA & 16,838 & 0.015 & 0.017 & 0.002 & 0.028 \\
\hline $\mathrm{ROA}$ & 16,704 & 0.009 & 0.017 & 0.000 & 0.020 \\
\hline$\delta$ & 16,838 & 0.333 & 0.471 & 0 & 1 \\
\hline \multicolumn{6}{|l|}{ Macroeconomic variables } \\
\hline GDP growth & 16,503 & 0.022 & 0.039 & -0.017 & 0.066 \\
\hline Interest rate & 14,747 & 0.039 & 0.058 & 0.014 & 0.063 \\
\hline \multicolumn{6}{|l|}{ Industry structure variables } \\
\hline HHI & 16,838 & 0.045 & 0.065 & 0.014 & 0.087 \\
\hline COMPTIER1 & 16,773 & 0.094 & 0.060 & 0.025 & 0.152 \\
\hline \multicolumn{6}{|l|}{ Bank type variables } \\
\hline Commercial dummy & 16,838 & 0.549 & 0.498 & 0 & 1 \\
\hline Savings dummy & 16,838 & 0.163 & 0.370 & 0 & 1 \\
\hline Government dummy & 16,838 & 0.094 & 0.291 & 0 & 0 \\
\hline Foreign dummy & 16,838 & 0.030 & 0.171 & 0 & 0 \\
\hline Subsidiary dummy & 16,838 & 0.094 & 0.292 & 0 & 0 \\
\hline \multicolumn{6}{|l|}{ Regulation variables } \\
\hline Overall capital stringency & 11,080 & 3.721 & 1.325 & 2 & 5 \\
\hline Deposit insurance & 16,838 & 0.765 & 0.935 & 0 & 2 \\
\hline Bail-out probability & 4,054 & 0.676 & 0.399 & 0 & 1 \\
\hline
\end{tabular}

Note. p10 and p90 indicate the 10th percentile and 90th percentile respectively.

\footnotetext{
34 Table B.2 in Appendix B gives a summary about the number of observations in our sample countries from 2001-2010.
} 
Table 2.2. Correlations Between Variables

\begin{tabular}{|c|c|c|c|c|c|c|c|c|c|c|c|c|}
\hline Variables & $\Delta \log G \mathrm{~L}$ & TIER1 & TIER2 & TCD & DEP & TA & LLP & FA & $\delta$ & GDP growth & Interest rate & TAXTA \\
\hline$\Delta \log G \mathrm{~L}$ & 1 & & & & & & & & & & & \\
\hline TIER1 & $-0.0290 * * *$ & 1 & & & & & & & & & & \\
\hline TIER2 & -0.00540 & $-0.0464 * * *$ & 1 & & & & & & & & & \\
\hline TCD & $-0.0131 *$ & $-0.179 * * *$ & $-0.0194 * *$ & 1 & & & & & & & & \\
\hline DEP & $0.0400 * * *$ & -0.0116 & $0.0938 * * *$ & $-0.504 * * *$ & 1 & & & & & & & \\
\hline TA & $-0.0437 * * *$ & $-0.235 * * *$ & $0.155 * * *$ & 0.00697 & $0.171 * * *$ & 1 & & & & & & \\
\hline LLP & $-0.0775^{* * *}$ & $-0.0308^{* * * *}$ & $0.0228 * * *$ & $-0.0161 * *$ & $0.0505^{* * *}$ & -0.000377 & 1 & & & & & \\
\hline FA & $0.0449 * * *$ & $0.0242 * * *$ & $0.0381 * * *$ & $0.0545^{* * *}$ & $-0.0778 * * *$ & $-0.234 * * *$ & $0.0458 * * *$ & 1 & & & & \\
\hline$\delta$ & $-0.0571 * * *$ & -0.00347 & $0.0453^{* * *}$ & $0.0256^{* * *}$ & $0.0613^{* * * *}$ & $0.0729 * * *$ & $0.0959 * * *$ & 0.00103 & 1 & & & \\
\hline GDP growth & $0.142 * * *$ & $0.0180^{* *}$ & $0.0416^{* * *}$ & $0.106^{* * *}$ & $0.0163 *$ & $0.0450 * * *$ & $-0.0298 * * *$ & $0.138^{* * * *}$ & $-0.363 * * *$ & 1 & & \\
\hline Interest rate & 0.0115 & $0.0448 * * *$ & $0.0166 * *$ & $-0.144 * * *$ & 0.000668 & $-0.0403 * * *$ & $0.0962 * * *$ & $0.0389 * * *$ & $0.0175^{* *}$ & 0.00311 & 1 & \\
\hline TAXTA & 0.00286 & $-0.0432 * * *$ & $0.0456 * * *$ & 0.00496 & 0.00136 & $0.348 * * *$ & -0.0115 & $-0.0678 * * *$ & $-0.0433 * * *$ & $0.0849 * * *$ & $-0.0271 * * *$ & 1 \\
\hline
\end{tabular}




\subsection{Empirical Estimation}

The empirical model is designed to test whether banks with different levels and quality of capital changed their lending behavior differently during the global financial crisis compared to the non-crisis period. The model is the following:

$\Delta \log G L_{i, t}=$ $\alpha_{i}+\beta \Delta \log G L_{i, t-1}+\left(\gamma+\gamma^{*} \delta_{t-1}\right) F U N D I N G_{i, t-1}+\left(\zeta+\zeta^{*} \delta_{t-1}\right)$ CONTROLS $_{i, t-1}+$ ${ }_{s M A C R O} \mathrm{MA}_{i, t}+\left(\eta+\eta^{*} \delta_{t-1}\right)$ COMPETITION $_{i, t-1}+\theta \delta_{t-1}$ BANK TYPE $_{i}+$ $\kappa \delta_{t-1}$ INSTITUTIONAL $_{i}+\lambda \delta_{t-1}$ Bailout probability $_{i}+\rho^{*} \delta_{t-1}+\varepsilon_{i, t-1}$

where:

$-\alpha$ is the intercept, $\beta, \lambda$, and $\rho$ are coefficients, and $\gamma, \gamma^{*}, \zeta, \zeta^{*}, \varsigma, \eta, \eta^{*}, \theta$, and $\kappa$ are coefficient vectors,

- $\Delta \log G L_{i, t}\left(\Delta \log G L_{i, t-1}\right)$ is the growth rate of the logarithm of gross loans of bank $i$ in year $t$ (in year $t-1$ ),

- $\delta_{t-1}$ denotes a dummy variable for the global financial crisis, which equals 1 in the period from 2008 to 2010 and 0 otherwise,

- FUNDING $G_{i, t-1}$ is a matrix of funding variables, including tier 1 capital ratio $\left(T I E R 1_{i, t-1}\right)$, tier 2 capital ratio $\left(T I E R 2_{i, t-1}\right)$, customer deposits to total assets $\left(T C D_{i, t-1}\right)$, and interbank deposits to total assets $\left(D E P_{i, t-1}\right)$,

- $\operatorname{CONTROLS}_{i, t-1}$ is a matrix of bank-specific control variables, including log total bank asset $\left(T A_{i, t-1}\right)$, loan loss provisions to total assets $\left(L L P_{i, t-1}\right)$, fixed assets to total assets $\left(F A_{i, t-}\right.$ $\left.{ }_{1}\right)$, and return on average assets $\left(R O A_{i, t-1}\right)$; only for $T A_{i, t-1}$ do we also include the interactive term with the crisis dummy,

- MACRO ${ }_{i, t}$ is a matrix of macroeconomic variables, including GDP growth $_{i, t}$ and Interest rate $_{i, t}$

- COMPETITION $N_{i, t-1}$ is a matrix of variables that describe industry structure, including the average tier 1 capital ratio of competing banks in a country $\left(C O M P T I E R 1_{i, t-1}\right)$ and Herfindahl-Hirschman Index $\left(H H_{i, t-1}\right)$,

- BANK TYPE $E_{i, t}$ is a matrix of bank characteristic dummies expressing ownership and organizational characteristics of banks, including Commercial dummy , Savings dummy $_{i}$, Government dummy ${ }_{i}$, Foreign dummy , and Subsidiary dummy $_{i}$,

- INSTITUTIONAL $i$ is a matrix of institutional and regulatory characteristics of bank $i$ 's country, including Overall capital stringency ${ }_{i}$, and Deposit insurance ${ }_{i}$,

- Bailout probability $i$ denotes the level of implicit government guarantees,

- $\varepsilon_{\mathrm{i}, \mathrm{t}}$ is an idiosyncratic error $\varepsilon_{\mathrm{i}, \mathrm{t}} \sim \operatorname{IID}\left(0, \delta_{\varepsilon}^{2}\right)$,

- $i=1,2, \ldots, N$ where $N$ is the number of banks in the sample,

- $t=1,2, \ldots, T_{i}$ where $T_{i}$ is the number of years in the sample for bank $i$.

We estimate three types of specifications. First, we use a fixed effects model with robust 
standard errors (that corresponds to (2.1) without the term $\Delta \log G L_{i, t-1}$ ). ${ }^{35}$ Second, we use an instrumental-variables estimator. We are concerned about the potential endogeneity of the tier 1 capital ratio in the fixed effects model. ${ }^{36}$ In particular, the fast growth of bank lending may not be due to a high tier 1 capital ratio, but because of other unidentified variables. For example, an efficient bank may easily build up a high tier 1 capital ratio (through retained earnings) and grow fast at the same time. In contrast, an inefficient bank grows slowly and is not able to build up a high level of capital. To deal with this endogeneity, we need to find valid instruments that are uncorrelated with the error term but correlated with our dependent variable.

We account for the potential endogeneity of our dependent variables $T I E R 1_{i, t-1}$, and $\delta_{t-1}$ TIER $1_{i, t-1}$ by instrumenting them with the first differences of TIER $1_{i, t-1}$ and $\delta_{t-1}$ TIER $1_{i, t-1}$ and crisis dummy $\delta_{t-1}$ and by $T A X T A_{t-1}$ (i.e., the ratio of tax values to bank size $\left.T A_{t-1}\right) .{ }^{37}$ We use the GMM estimator to obtain consistent and efficient estimates in the presence of non-i.i.d. errors (see Baum (2006)). Following Driscoll and Kraay (1998), we employ a cluster-robust estimator (where clusters are defined at the level of banks) to account for within-cluster correlation of the disturbances.

The instruments are statistically significant at large in first-stage regression equations. In particular, TIER $1_{i, t-1}$ is statistically significant (at $1 \%$ ) and positively related to the first difference of $T I E R 1_{i, t-1}$ and negatively related (at $5 \%$ statistical significance) to the first difference of $\delta_{t-1} . \delta_{t-1} T I E R 1_{i, t-1}$ is statistically significantly (at $1 \%$ ) and positively related to the first difference of $\delta_{t-1} T I E R 1_{i, t-1}$ and negatively related (at $5 \%$ statistical significance) to the first difference of $\delta_{t-1}$. In addition, tests for underidentification and weak identification (measured by the Kleibergen-Paap rk LM and Wald F statistic, Kleibergen and Paap (2006), and by the Cragg-Donald Wald F statistic, see Hall, Rudebusch, and Wilcox (1996); see also Hall and Peixe (2000) ) confirm the validity of the instruments chosen.

Third, we use the dynamic panel-data setup to account for potential endogeneity of our dependent variable $\Delta \log G L_{i, t}$. If lagged loan growth $\Delta \log G L_{i, t-1}$ is correlated with the panel-level effects, the estimator may become inconsistent. We employ the two-step generalized method of moments (GMM) procedure of Arellano and Bond (1991). Our sample has a short time dimension and large country dimension. The Arellano-Bond estimator is particularly useful in obtaining unbiased and efficient estimates in short dynamic panels with lagged endogenous variables as an explanatory variable. We employ a cluster-robust estimator to account for potentially non-i.i.d. errors and to obtain consistent standard error estimates even in the presence of autocorrelation or heteroskedasticity within panels. We use $\Delta \log G L_{i, t-1}, T I E R 1_{i, t-1}$ and $\delta_{t-1}$ TIER $1_{i, t-1}$ as endogenous instruments and all other regressors as exogenous instruments

\footnotetext{
35 The Hausman test indicates that a fixed effects model should be used rather than a random effects model.

36 The bank capital structure decision is endogenous and may depend on bank-specific variables and macroeconomic and regulatory conditions in a country (see Byoun (2008); Gropp and Heider (2010); Flannery and Rangan (2008); Memmel and Raupach (2010)).

37 Ashcraft (2008b) and Berger and Bouwman (2013) also employ tax rate as an instrumental variable. Alternatively, Billmeier and Nannicini (2013) employ a synthetic control approach to control for endogeneity issues in a cross-country study.
} 
(in line with Roodman (2009). We use sets of lags (from 2 to 6) to mitigate the overidentification problem of endogenous instruments.

\subsection{Results}

We start by looking at the basic setup, where 'supply'-side credit factors (capital and deposits), individual bank controls (size, loan-loss provisions, tangibility), and 'demand'-side credit factors (economic growth, interest rates) are included. We continue by adding 1) industry competition factors (concentration, capitalization of competitors), 2) bank ownership characteristics (foreign vs. domestic ownership, subsidiary status), 3) institutional and regulatory characteristics (stringency of capital regulation and the coverage of deposit insurance), and 4) indirect government support (bail-out probability).

'Supply'-side (funding) factors and credit growth: In the basic model (columns 1, 6, and 11 in Table 2.3), we evaluate the impact of credit 'supply'-side variables (capital quality and funding) and the effect of financial crisis on credit growth (lending behavior) in the total sample of banks.

The results reported in columns $1-5$ in Table 2.3 indicate that the tier 1 coefficient is statistically significant at the $1 \%$ level. This points to a positive relationship between the highest quality bank capital $T I E R_{1, t-1}$ and the credit growth $\Delta \log G L_{t}$ and it confirms our Hypothesis 2.1. Moreover, the interaction term constructed as a product of the tier 1 ratio and crisis dummy also demonstrates a positive relationship with loan growth. This supports the notion of tier 1 serving as a buffer and not an incentive mechanism for banks and is consistent with Hypothesis 2.1.

Tier 1 capital provides banks with a cushion to absorb banks' losses and insulates banks from the risk of bankruptcy. Banks with higher tier 1 capital ratio levels are less sensitive to their actual credit and liquidity risk exposures. Consequently, they could secure market funding and support credit growth even during the global financial crisis. In contrast, banks with a low tier 1 capital ratio may face serious solvency and liquidity problems if a recession looms. Consequently, they needed to heavily cut back on lending in the global financial crisis. The positive effect of tier 1 capital ratio on credit growth and the reinforced effect during the financial crisis is consistent with Jiménez et al. (2012), who find that the banks, especially those with lower capital ratio levels, have a negative growth in lending activity.

Columns 6-10 in Table 2.3 present the results using the instrumental variables panel regression model. The main difference from the previous columns in Table 2.3 is that the coefficient tier 1 capital ratio becomes insignificant. However, the interaction term between tier 1 capital ratio and the crisis dummy is consistently highly significant and positively related to lending growth. This provides evidence that tier 1 capital was especially important in the global financial crisis, whereas it is not significantly related to bank lending in normal times. We assess the adequacy of instruments using a test of overidentifying restrictions. We employ Hansen's J statistic, which is robust to heteroskedasticity and autocorrelation issues. $P$-values of Hansen's $\mathrm{J}$ statistic show that we cannot reject the null hypothesis that the instruments are uncorrelated with the error term. This points to the validity of the instruments. In columns 11-15 in Table 2.3 we report the results 
of the difference GMM model. Hansen's J statistic again confirms the validity of the instruments. The signs and statistical significance of the estimated coefficients largely correspond to the previous columns in Table 2.3.

Next, we turn to the effect of tier 2 capital on credit growth. We can find some evidence that tier 2 capital positively affects lending growth in normal times. Contrary to our expectations in Hypothesis 2.2, tier 2 capital had no significant effect on credit growth during the global financial crisis.

We also find some evidence that the type of bank deposits affects lending. In particular, customer deposits $\left(T C D_{i, t-1}\right)$ positively and significantly at $1 \%$ affected bank lending during the global financial crisis in almost all empirical specifications. This is in line with Hypothesis 2.3 and the view that (mainly insured) customer deposits acted as a stable source of funding during the global financial crisis. Our analysis also provides some evidence that interbank lending $\left(D E P_{i, t-1}\right)$ is positively associated with bank lending during normal times and negatively during the global financial crisis, although the statistical significance is less pervasive across different empirical specifications (i.e., the negative signs of bank deposits during the global financial crisis $\delta_{t-1} D E P_{i, t-1}$ become significant when using the difference GMM model). This is consistent with our Hypothesis 2.3 .

Looking at bank-specific control variables, the regression result suggests that bank size $\left(T A_{i, t-1}\right)$ affects bank lending behavior. The negative and significant $T A_{i, t-1}$ coefficient indicates that in normal times larger banks experience lower credit growth rates than small banks. Other control variables are less statistically significant.

'Demand'-side factors and credit growth: In order to capture the effect of 'demand' side factors on credit growth, we include annual GDP growth (GDP growth $\left._{i, t}\right)$ and real interest rates to prime customers (Interest rate $_{i, t}$ ) in the model in Table 2.3. Not surprisingly, the results suggest that credit growth is negatively correlated with the interest rate and positively correlated with GDP growth ${ }_{i, t}{ }^{38}$

Banking sector competition and credit growth: The effect of funding factors on credit growth may be driven by the level of competition and the overall structure of the banking industry. We therefore add a concentration variable $\left(H H I_{i, t-1}\right)$, the average capitalization of the competitors $\left(\right.$ COMPTIER $\left.1_{i, t-1}\right)$, and their interactive terms with a crisis dummy $\left(\delta_{t-1} H H I_{i, t-1}\right.$ and $\delta_{t-1}$ COMPTIER $1_{i, t-1}$ ) as additional explanatory variables to the basic model (columns 2 , 7 , and 12 in Table 2.3).

We observe a negative effect of the concentration index $\left(H H I_{i, t-1}\right)$ on credit growth (although

\footnotetext{
${ }^{38} \mathrm{We}$ also tested for alternative empirical specifications, which include a yearly change in interest rate and its interactive term with the crisis dummy as additional explanatory variables. Their impact on bank lending behavior was statistically insignificant in normal times and during the global financial crisis. Our other results remain largely unchanged. This confirms the importance of tier 1 capital put forward within the lending channel literature (see Gambacorta and Marques-Ibanez (2011)).
} 
mostly insignificant) during normal times. Hence, we find very scant support for Hypothesis 2.4a. However, competitors' tier 1 capital ratios have a significantly positive impact on loan growth during normal times (i.e., the coefficient for COMPTIER $1_{i, t-1}$ is significantly positive), but this coefficient reversed during the global financial crisis. This points to the competitive advantage of high tier 1 capital ratios in the global financial crisis. In particular, banks grew more slowly in the global financial crisis if their competitors had high tier 1 ratios. In contrast, banks grow faster in normal times if their competitors have high tier 1 ratios. In normal times, high tier 1 ratios may act as a competitive disadvantage. This provides support only for Hypothesis 2.4b.

The results regarding the effect of the capital and deposits on credit growth are both qualitatively and quantitatively very similar to the basic model. This implies that our basic results are robust for the inclusion of the industry competition measures.

Regulatory environment: The relationship between bank capital and bank lending behavior may be driven by cross-country differences, especially in the regulatory and institutional framework. We control for country-specific regulatory variables by adding measures of capital regulation stringency and deposit insurance. The relationship between funding variables $\left(T I E R 1_{i, t-1}\right)$ and bank lending remains unchanged. Our results provide some evidence that banks cut back on lending more in the global financial crisis if capital stringency in the country was more pronounced. However, when using the instrumental variables for panel regression or difference GMM estimation, the regulation variable Overall capital stringency loses its statistical significance. The signs and statistical significance of other estimated coefficients largely correspond with the ones in the basic model specification.

Bank ownership and credit growth: Hypotheses 2.5a, 2.5b, and 2.5c are related to the effect of various ownership aspects on credit growth during the global financial crisis. We therefore include as dependent variables dummy variables related to organizational structure and ownership of individual banks multiplied by the crisis dummy (see columns 3, 7, and 13 in Table 2.3). We find a significant and positive effect of government ownership on credit growth in the global financial crisis. This corresponds to the direct support of governments through ownership participation in banks and also confirms our Hypothesis 2.5a. Government-owned banks could tap funding and support from governments during the global financial crisis and continue with their loan growth.

We also find some limited support for the negative effect of foreign ownership on lending growth during the global financial crisis. This is in line with Hypothesis 2.5b. Finally, we find limited evidence that credit growth during the global financial crisis was affected by the organizational structure of the bank. That is, we find that a subsidiary bank cut back on lending less during the global financial crisis than a stand-alone entity. This is in line with our Hypothesis 2.5c. Finally, we observe that the results regarding the effect of tier 1 capital on credit growth are both qualitatively and quantitatively very similar to the basic model. This implies that our basic results are robust for controlling for the bank organizational structure and 
ownership.

Indirect government support and the credit growth: During the financial crisis, banks were largely supported by governments. It is therefore important to control for the impact of implicit government guarantees on lending activities. For this purpose, we include the variable Bailout probability. We extend the basic model by including the interaction terms of Bailout probability with a crisis dummy. The results mainly show an insignificant effect of Bailout probability on credit growth during the global financial crisis. More importantly, the effects of capital and deposits on credit growth remain qualitatively similar to those in the basic model. 
Table 2.3. Estimation Result of Total Sample

\begin{tabular}{|c|c|c|c|c|c|c|c|c|c|c|c|c|c|c|c|}
\hline & (1) & (2) & (3) & (4) & (5) & (6) & (7) & (8) & (9) & (10) & (11) & (12) & (13) & (14) & (15) \\
\hline \multicolumn{16}{|c|}{ Bank specific variables } \\
\hline \multirow[t]{2}{*}{ Intercept } & $2.871^{* * * *}$ & $2.999^{* * * *}$ & $2.861^{* * *}$ & $2.765^{* * *}$ & $2.519^{* * *}$ & & & & & & & & & & \\
\hline & $(6.37)$ & $(6.32)$ & (6.40) & (4.91) & $(3.34)$ & & & & & & & & & & \\
\hline \multirow[t]{2}{*}{$\Delta \log G L_{i, t-1}$} & & & & & & & & & & & -0.265 & $-0.454^{* * *}$ & -0.296 & $-0.414^{*}$ & $-0.292^{*}$ \\
\hline & & & & & & & & & & & $(-1.42)$ & $(-2.65)$ & $(-1.60)$ & $(-1.73)$ & $(-1.87)$ \\
\hline \multirow[t]{2}{*}{ TIER $1_{\mathrm{i}, t-1}$} & $0.400^{* * * *}$ & $0.392^{* * *}$ & $0.402^{* * *}$ & $0.379^{* * *}$ & $0.510^{* * *}$ & 0.404 & 0.392 & 0.443 & 0.392 & 0.488 & -0.0028 & -0.0984 & 0.0208 & 0.0806 & 0.195 \\
\hline & (3.46) & (3.37) & $(3.50)$ & (2.74) & (2.77) & (1.32) & (1.28) & (1.44) & (1.01) & (1.03) & $(-0.01)$ & $(-0.18)$ & $(0.04)$ & $(0.09)$ & $(0.98)$ \\
\hline \multirow[t]{2}{*}{$\operatorname{TIER} 1_{\mathrm{i}, \mathrm{t}-\mathrm{l}} * \delta_{\mathrm{t}-1}$} & $0.310^{* * * *}$ & $0.317^{* * *}$ & $0.297^{* * *}$ & $0.423^{* * *}$ & $0.369^{* * * *}$ & $0.230^{* *}$ & $0.238^{* * *}$ & $0.211^{* *}$ & $0.324^{* * *}$ & $0.253^{* * *}$ & $0.513^{* * * 8}$ & $0.479^{* * * *}$ & $0.479^{* * * *}$ & $0.483^{* *}$ & $0.203^{*}$ \\
\hline & $(3.00)$ & (3.15) & $(3.05)$ & (4.91) & (3.82) & (2.54) & (2.75) & (2.34) & (4.05) & $(2.82)$ & (3.37) & (2.95) & $(2.90)$ & (1.99) & (1.89) \\
\hline \multirow[t]{2}{*}{$\operatorname{TIER} 2_{\mathrm{i},-1}$} & $0.639^{* * * *}$ & $0.623^{* * * *}$ & $0.624^{* * *}$ & $0.630^{* *}$ & $1.484^{* * * *}$ & $0.517^{* *}$ & $0.499^{*}$ & $0.510^{* *}$ & $0.560^{*}$ & $1.034^{*}$ & 0.318 & 0.187 & 0.332 & 0.356 & 0.132 \\
\hline & (2.72) & $(2.67)$ & (2.68) & $(2.50)$ & $(2.77)$ & $(2.00)$ & (1.95) & (1.97) & $(1.82)$ & (1.95) & $(0.91)$ & $(0.51)$ & $(0.90)$ & $(0.49)$ & $(0.27)$ \\
\hline \multirow[t]{2}{*}{$\operatorname{TIER} 2_{\mathrm{i}, \mathrm{t}-\mathrm{s}} * \delta_{\mathrm{t}-1}$} & 0.271 & 0.159 & 0.364 & 0.654 & -0.912 & 0.212 & 0.0624 & 0.361 & 0.302 & -0.896 & 0.616 & 0.298 & 0.475 & 0.343 & 0.294 \\
\hline & $(0.48)$ & $(0.28)$ & $(0.65)$ & $(1.22)$ & $(-1.01)$ & $(0.33)$ & $(0.10)$ & $(0.56)$ & $(0.52)$ & $(-0.96)$ & (1.10) & $(0.53)$ & $(0.84)$ & $(0.55)$ & $(0.55)$ \\
\hline \multirow[t]{2}{*}{$\mathrm{TCD}_{\mathrm{i},-1-1}$} & 0.152 & 0.136 & 0.157 & 0.0553 & 0.0955 & 0.204 & 0.171 & 0.224 & 0.0617 & 0.00434 & 0.14 & 0.152 & 0.14 & 0.115 & 0.068 \\
\hline & (1.26) & (1.12) & (1.29) & (0.36) & $(0.51)$ & (1.50) & (1.23) & (1.64) & (0.34) & $(0.02)$ & $(0.92)$ & $(0.96)$ & $(0.88)$ & $(0.39)$ & $(0.51)$ \\
\hline \multirow[t]{2}{*}{$\mathrm{TCD}_{\mathrm{i},-1-1} * \delta_{\mathrm{t}-1}$} & $0.168^{* * * *}$ & $0.166^{* * * *}$ & $0.144^{* * *}$ & $0.216^{* * *}$ & $0.190^{* * * *}$ & $0.201^{* * * *}$ & $0.202^{* * * *}$ & $0.189^{* * * *}$ & $0.230^{* * * *}$ & $0.218^{* *}$ & $0.104^{* *}$ & $0.102^{* *}$ & 0.0623 & 0.137 & $0.184^{3 *}$ \\
\hline & (3.86) & (3.80) & (2.58) & (3.10) & (2.70) & $(4.13)$ & $(4.13)$ & $(2.85)$ & $(2.75)$ & $(2.38)$ & $(2.11)$ & $(2.10)$ & $(0.88)$ & $(1.10)$ & $(2.23)$ \\
\hline \multirow[t]{2}{*}{$\operatorname{DEP}_{\mathrm{i}, t-1}$} & $0.354^{* *}$ & $0.338^{* *}$ & $0.352^{* *}$ & $0.477^{* *}$ & 0.138 & $0.340^{*}$ & $0.309^{*}$ & $0.364^{*}$ & 0.378 & 0.019 & 0.208 & 0.334 & 0.21 & 0.483 & -0.167 \\
\hline & (2.07) & (1.97) & $(2.01)$ & (2.14) & $(0.70)$ & (1.85) & (1.68) & (1.94) & (1.53) & $(0.07)$ & $(0.71)$ & (1.08) & $(0.72)$ & (1.04) & $(-0.73)$ \\
\hline \multirow[t]{2}{*}{$\operatorname{DEP}_{\mathrm{i}, \mathrm{t}-1} * \delta_{t-1}$} & -0.288 & -0.301 & -0.286 & -0.421 & -0.0208 & -0.182 & -0.164 & -0.207 & -0.122 & 0.0429 & $-0.598^{* *}$ & $-0.705^{* * *}$ & $-0.553^{*}$ & -0.66 & 0.0381 \\
\hline & $(-1.54)$ & $(-1.62)$ & $(-1.26)$ & $(-1.26)$ & $(-0.14)$ & $(-0.81)$ & $(-0.73)$ & $(-0.78)$ & $(-0.31)$ & $(0.24)$ & $(-2.49)$ & $(-2.92)$ & $(-1.91)$ & $(-1.33)$ & $(0.18)$ \\
\hline \multirow[t]{2}{*}{$\mathrm{TA}_{\mathrm{i}, t-1}$} & $-0.209^{* * * *}$ & $-0.217^{* * *}$ & $-0.209^{* * * *}$ & $-0.191^{* * * *}$ & $-0.163^{* * *}$ & $-0.189^{* * * *}$ & $-0.200^{* * *}$ & $-0.183^{* * *}$ & $-0.160^{* * * *}$ & $-0.153^{* * * *}$ & $-0.345^{* * *}$ & $-0.310^{* * *}$ & $-0.342^{2 * *}$ & $-0.284^{* * * *}$ & $-0.240^{s * * t}$ \\
\hline & $(-7.20)$ & $(-7.17)$ & $(-7.24)$ & $(-5.68)$ & $(-3.84)$ & $(-5.20)$ & $(-5.28)$ & $(-5.28)$ & $(-3.72)$ & $(-3.86)$ & $(-5.77)$ & $(-4.74)$ & $(-5.57)$ & $(-4.25)$ & $(-3.90)$ \\
\hline \multirow[t]{2}{*}{$\mathrm{TA}_{\mathrm{i}, \mathrm{t}-1} * \delta_{\mathrm{t}-1}$} & 0.000507 & 0.00269 & -0.00394 & 0.00545 & 0.00498 & -0.00425 & -0.00105 & -0.0078 & 0.00884 & 0.00277 & 0.00627 & $0.00992^{*}$ & 0.00416 & 0.0145 & -0.00417 \\
\hline & $(0.11)$ & $(0.54)$ & $(-0.61)$ & $(0.56)$ & $(0.62)$ & $(-0.81)$ & $(-0.19)$ & $(-1.01)$ & $(0.94)$ & $(0.28)$ & (1.19) & (1.67) & $(0.54)$ & $(0.88)$ & $(-0.49)$ \\
\hline \multirow[t]{2}{*}{$\operatorname{LLP}_{\mathrm{i},-1-1}$} & -1.102 & -1.106 & -1.107 & -1.267 & -0.512 & -0.48 & -0.37 & -0.446 & -0.489 & 0.191 & -1.095 & -1.079 & -0.997 & -0.753 & -0.388 \\
\hline & $(-1.45)$ & $(-1.47)$ & $(-1.46)$ & $(-1.60)$ & $(-0.65)$ & $(-0.52)$ & $(-0.41)$ & $(-0.49)$ & $(-0.49)$ & $(0.24)$ & $(-1.08)$ & $(-1.09)$ & $(-0.97)$ & $(-0.50)$ & $(-1.05)$ \\
\hline \multirow[t]{2}{*}{$\mathrm{FA}_{\mathrm{i}, t-1}$} & 0.697 & 0.676 & 0.683 & 0.944 & $2.086^{* *}$ & 1.067 & 1.012 & 1.056 & 1.328 & $2.245^{* *}$ & 0.401 & 0.103 & 0.345 & 0.805 & 0.638 \\
\hline & (1.15) & (1.13) & (1.12) & $(1.31)$ & $(2.20)$ & (1.48) & (1.43) & $(1.47)$ & (1.54) & (2.39) & $(0.59)$ & $(0.15)$ & $(0.50)$ & $(0.90)$ & $(0.62)$ \\
\hline \multirow[t]{2}{*}{$\mathrm{ROA}_{\mathrm{i},-1-1}$} & 0.363 & 0.393 & 0.35 & 0.349 & $1.131^{* *}$ & 0.595 & 0.592 & 0.576 & 0.485 & $1.162^{*}$ & -0.203 & 0.0604 & -0.121 & 0.0622 & 0.674 \\
\hline & $(0.59)$ & $(0.65)$ & $(0.58)$ & $(0.55)$ & $(2.08)$ & $(0.80)$ & $(0.80)$ & $(0.78)$ & $(0.61)$ & (1.81) & $(-0.33)$ & $(0.11)$ & $(-0.21)$ & $(0.10)$ & $(0.96)$ \\
\hline \multirow[t]{2}{*}{$\delta_{\mathrm{t}-1}$} & -0.103 & -0.0964 & -0.0314 & -0.169 & -0.141 & -0.052 & -0.0577 & 0.00229 & -0.273 & -0.114 & $-0.192^{* * *}$ & -0.171 & -0.148 & -0.305 & -0.0539 \\
\hline & $(-1.59)$ & $(-1.32)$ & $(-0.32)$ & $(-0.81)$ & $(-0.89)$ & $(-0.71)$ & $(-0.72)$ & $(0.02)$ & $(-1.34)$ & $(-0.56)$ & $(-2.27)$ & $(-1.62)$ & $(-1.13)$ & $(-0.88)$ & $(-0.30)$ \\
\hline \multicolumn{16}{|c|}{ Macroeconomic variables } \\
\hline \multirow{2}{*}{$\frac{\text { Macroeconomic }}{\text { GDP growth }_{\mathrm{i}, \mathrm{t}}}$} & $0.692^{\text {wat }}$ & $0.739^{n * * *}$ & $0.725^{m+m}$ & $0.865^{* * *}$ & $0.652^{2 * 2 *}$ & $0.641^{m+m}$ & $0.729^{\text {n*we }}$ & $0.657^{m+m}$ & $0.856^{* 2 *}$ & $0.694^{2 \times 4}$ & $0.535^{2 * m}$ & $0.518^{n+m e}$ & $0.571^{* 2 *}$ & $0.759^{m+m}$ & $0.493^{* 2 * *}$ \\
\hline & $(4.52)$ & $(4.76)$ & (4.44) & $(4.80)$ & (3.42) & (3.81) & (4.18) & (3.59) & $(4.27)$ & (3.50) & (3.04) & (2.77) & (3.11) & (2.91) & $(2.61)$ \\
\hline \multirow{2}{*}{ Interest rate $\mathrm{e}_{\mathrm{i}, \mathrm{t}}$} & $-0.599^{* * *}$ & $-0.639^{* * * *}$ & $-0.590^{* * *+}$ & $-0.540^{* 3 * *}$ & $-0.394^{* * *}$ & $-0.471^{* * *}$ & $-0.522^{* * *}$ & $-0.468^{* * t}$ & $-0.372^{* *}$ & -0.218 & $-0.458^{* * *}$ & $-0.477^{* * s}$ & $-0.451^{* * * *}$ & -0.229 & -0.0951 \\
\hline & $(-4.75)$ & $(-4.64)$ & $(-4.17)$ & $(-3.76)$ & $(-2.69)$ & $(-3.41)$ & $(-3.30)$ & $(-3.00)$ & $(-2.33)$ & $(-1.59)$ & $(-3.81)$ & $(-3.46)$ & $(-3.17)$ & $(-1.47)$ & $(-0.80)$ \\
\hline \multicolumn{16}{|c|}{ Industry structure variables } \\
\hline
\end{tabular}


Table 2.3. Estimation Result of Total Sample

\begin{tabular}{|c|c|c|c|c|c|c|c|c|c|c|c|c|c|c|c|}
\hline & (1) & (2) & (3) & (4) & (5) & (6) & (7) & (8) & (9) & (10) & (11) & (12) & (13) & (14) & (15) \\
\hline \multirow[t]{2}{*}{$\mathrm{HHI}_{\mathrm{i}, \mathrm{L}}$} & & $-0.684^{*}$ & & & & & -1.01 & & & & & $\begin{array}{c}-0.673 \\
\end{array}$ & & & \\
\hline & & $(-1.66)$ & & & & & $(-1.61)$ & & & & & $(-1.29)$ & & & \\
\hline \multirow{2}{*}{$\mathrm{HHI}_{\mathrm{i},-1-1} * \delta_{\mathrm{t}-1}$} & & 0.239 & & & & & 0.407 & & & & & -0.0689 & & & \\
\hline & & (1.16) & & & & & (1.52) & & & & & $(-0.27)$ & & & \\
\hline \multirow[t]{2}{*}{ COMPTIER $1_{i,-1}$} & & $0.303^{* * *}$ & & & & & $0.394^{* * * *}$ & & & & & $0.445^{* * * *}$ & & & \\
\hline & & $(3.05)$ & & & & & $(3.82)$ & & & & & $(3.81)$ & & & \\
\hline \multirow[t]{2}{*}{ COMPTIER $1_{1, t-1} * \delta_{t-1}$} & & $-0.467^{* * *}$ & & & & & $-0.585^{* * *}$ & & & & & $-0.635^{* * *}$ & & & \\
\hline & & $(-3.53)$ & & & & & $(-4.12)$ & & & & & $(-4.62)$ & & & \\
\hline \multicolumn{16}{|l|}{ Bank type variables } \\
\hline \multirow{2}{*}{ Commercial dummy $* \delta_{t-1}$} & & & 0.0000956 & & & & & -0.00187 & & & & & -0.00101 & & \\
\hline & & & $(0.00)$ & & & & & $(-0.04)$ & & & & & $(-0.02)$ & & \\
\hline \multirow[t]{2}{*}{ Savings dummy $* \delta_{t-1}$} & & & 0.00412 & & & & & -0.00333 & & & & & 0.0373 & & \\
\hline & & & $(0.13)$ & & & & & $(-0.10)$ & & & & & $(0.92)$ & & \\
\hline \multirow[t]{2}{*}{ Government dummy $* \delta_{t-1}$} & & & $0.0492^{* *}$ & & & & & 0.0314 & & & & & $0.0560^{* *}$ & & \\
\hline & & & (2.50) & & & & & $(1.33)$ & & & & & $(2.36)$ & & \\
\hline \multirow[t]{2}{*}{ Foreign dummy $* \delta_{\mathrm{t}-1}$} & & & -0.071 & & & & & $-0.105^{*}$ & & & & & -0.0266 & & \\
\hline & & & $(-1.51)$ & & & & & $(-1.94)$ & & & & & $(-0.57)$ & & \\
\hline \multirow[t]{2}{*}{ Subsidiary dummy $* \delta_{t-1}$} & & & 0.0256 & & & & & $0.0451^{* *}$ & & & & & $0.0384^{*}$ & & \\
\hline & & & $(1.27)$ & & & & & $(1.99)$ & & & & & $(1.74)$ & & \\
\hline \multicolumn{16}{|l|}{ Regulation variables } \\
\hline \multirow[t]{2}{*}{ Overall capital stringency $* \delta_{t-1}$} & & & & $-0.0173^{*}$ & & & & & -0.0106 & & & & & -0.011 & \\
\hline & & & & $(-1.87)$ & & & & & $(-1.00)$ & & & & & $(-0.79)$ & \\
\hline \multirow[t]{2}{*}{ Deposit insurance $* \delta_{t-1}$} & & & & 0.0106 & & & & & 0.021 & & & & & -0.016 & \\
\hline & & & & $(0.39)$ & & & & & $(0.70)$ & & & & & $(-0.38)$ & \\
\hline \multirow[t]{2}{*}{ Bail-out probability * $\delta_{t-1}$} & & & & & -0.0582 & & & & & $-0.0647^{*}$ & & & & & -0.0311 \\
\hline & & & & & $(-1.59)$ & & & & & $(-1.71)$ & & & & & $(-0.67)$ \\
\hline$N$ & 7375 & 7374 & 7375 & 3903 & 2048 & 5655 & 5655 & 5655 & 2631 & 1626 & 4538 & 4538 & 4538 & 2317 & 1379 \\
\hline Adjusted-R2 & 0.168 & 0.176 & 0.171 & 0.208 & 0.239 & & & & & & & & & & \\
\hline Hansen J Statistic ( $p$-value) & & & & & & $3.591(0.166)$ & $3.377(0.185)$ & $4.103(0.129)$ & $4.271(0.118)$ & $0.848(0.654)$ & $17.11(0.146)$ & $16.08(0.187)$ & $17.1(0.146)$ & $16.9(0.153)$ & $38.26(0.0935)$ \\
\hline Estimation Method & $\mathrm{FE}$ & $\mathrm{FE}$ & $\mathrm{FE}$ & $\mathrm{FE}$ & $\mathrm{FE}$ & IV & IV & IV & IV & IV & GMM & GMM & GMM & GMM & GMM \\
\hline
\end{tabular}




\subsection{Robustness Checks}

Subsamples of commercial banks: As a robustness check we also performed an analysis on the subsample of commercial banks only (see Appendix B Table B.3). The results were largely unchanged. The crisis dummy is negatively and in most specifications significantly related to bank lending. This confirms the view that commercial banks needed to cut back on lending during the global financial crisis. The concentration index $(H H I)$ also has a more significant impact on bank lending behavior, as predicted by Hypothesis 2.4a.

Subsamples of Banks in Different Regions: Despite having wide-reaching effects on the global economy, the global financial crisis affected developed and developing countries differently. It is therefore interesting and warranted to perform the same analysis on the subsamples of banks in OECD, non-OECD, and BRIC countries. In particular, we are interested in whether the impact of tier 1 capital on bank lending is unchanged in different world regions. ${ }^{39}$

Table 2.4 reports the results in each region based on the fixed effects model, instrumental variable regression model, and Arellano and Bond's (1991) GMM estimation.

In the subsample of banks in the OECD countries, the impact of the tier 1 capital ratio and its interaction term with the crisis dummy is fairly insignificant. Within the subsample of non-OECD countries and BRIC countries, however, the impact of the tier 1 capital ratio on bank lending during the global financial crisis is positive and significant among all model specifications. This shows that during the global financial crisis, a high tier 1 capital ratio was especially important for bank lending in developing countries (i.e., non-OECD and BRIC countries).

Interestingly, the role of government ownership for bank lending during the global financial crisis was positive and statistically significant only for banks in the non-OECD and BRIC subsamples. This may indicate that government ownership helps banks weather the global financial crisis only in developing countries.

Subsample of Banks According to Size and Funding: The size of a bank plays a significant role in its ability to access financial markets in order to secure various types of funding for its operations, as well as to sufficiently diversify the riskiness of its assets and achieve certain economies of scale and scope. The question arises whether our findings are limited to the subsample of banks with a high (or low) tier 1 and tier 2 capital ratio, customer deposits, and interbank deposits. We therefore replicate our analysis on the subsamples of large and small banks by total assets (columns 1 and 2), banks with a high and low tier 1 capital ratio (columns 3 and 4), banks with a high and low tier 2 capital (columns 5 and 6), banks with high and low customer deposits (columns 7 and 8), and banks with high and low interbank deposits (columns 9 and 10). We report

\footnotetext{
39 Brewer, Kaufman, and Wall (2008), for example, show that capital ratios of banks not only depend on bank-specific variables, but also on country characteristics and policy variables. In addition, Berger et al. (2008) demonstrate that U.S. bank holding companies actively manage their capital ratios. One can therefore expect the relationship between capital and credit growth to be country-specific as well.
} 
the results in Table 2.5. For the purpose of brevity, we limit our estimation method to the fixed effects model.

Our analysis shows that the tier 1 capital ratio and its interaction with the crisis dummy is statistically significant for smaller banks, but not for the largest banks. Small banks may be driven mostly by market forces and the impact of the global financial crisis on small banks may have been the highest. Therefore, high tier 1 capital ratio levels are the most important for small banks. In contrast, large banks may have been partially shielded from the global financial crisis by implicit government guarantees. Their too-big-to-fail status may lower the importance of the tier 1 capital ratio.

In addition, the tier 1 capital ratio and its interaction term with the crisis dummy significantly affect bank lending for banks with a high tier 1 ratio, low tier 2 ratio, low customer deposits-to-total asset ratio, and low interbank deposits-to-total asset ratio. This confirms the finding by Brei et al. (2013), who show that bank capital supports lending, but only if it surpasses a critical threshold. Our analysis brings in the size effect. Looking back at Table 2.2, it can also be noted that size is negatively correlated with TIERI and positively correlated with TIER2, TCD, and DEP. Table 2.5 therefore indicates that the tier 1 ratio was important for bank lending behavior during the global financial crisis, especially for small banks.

Interestingly, the competitive environment significantly affects the lending behavior of large banks but not small banks. COMPTIER 1 is significantly and positively related - and COMPTIERI $\delta_{t}$ is significantly and negatively related - to lending growth for large banks (and for banks with low tier 1 and high tier 2, TCD but low $D E P$ ), but its relation becomes insignificant for small banks (and for banks with a high tier 1 and low tier 2 capital ratio and TCD but with high $D E P$ ). That is, in normal times, large banks grow significantly faster if competing banks in the country have high tier 1 capital ratio, whereas during the global financial crisis large banks grew significantly faster if competing banks had a low tier 1 capital ratio. The explanation may be that high tier 1 capital ratio acted as a deterrent against the growth of large banks during the global financial crisis, but acts as a competitive disadvantage during normal times. 
Table 2.4. Estimation Result of Subsamples by Different Regions

Bank specific variables

Intercept

$\Delta \log \mathrm{GL}_{\mathrm{i}, \mathrm{t}-1}$

TIER $1_{i, t-1}$

TIER $1_{\mathrm{i}, \mathrm{t}-1} * \delta_{\mathrm{t}-1}$

TIER $2_{\mathrm{i}, \mathrm{t}-1}$

TIER $_{\mathrm{i}, \mathrm{t}-1} * \delta_{\mathrm{t}-1}$

$\mathrm{TCD}_{\mathrm{i}, \mathrm{t}-1}$

$\mathrm{TCD}_{\mathrm{i}, \mathrm{t}-1} * \delta_{\mathrm{t}-1}$

$\operatorname{DEP}_{i, t-1}$

$\mathrm{DEP}_{\mathrm{i}, \mathrm{t}-1} * \delta_{\mathrm{t}-1}$

$\mathrm{TA}_{\mathrm{i}, \mathrm{t}-1}$

$\mathrm{TA}_{\mathrm{i}, \mathrm{t}-1} * \delta_{\mathrm{t}-1}$

$\mathrm{LLP}_{\mathrm{i}, \mathrm{t}-1}$

$\mathrm{FA}_{\mathrm{i}, \mathrm{t}-1}$

$\mathrm{ROA}_{\mathrm{i}, \mathrm{t}-1}$

$\delta_{\mathrm{t}-1}$

\begin{tabular}{|c|c|c|c|c|c|c|c|c|}
\hline \multicolumn{3}{|c|}{ OECD Subsample } & \multicolumn{3}{|c|}{ Non-OECD Subsample } & \multicolumn{3}{|c|}{ BRIC Subsample } \\
\hline$(1)$ & $(2)$ & (3) & $(1)$ & $(2)$ & (3) & $(1)$ & $(2)$ & (3) \\
\hline $\begin{array}{c}3.448^{\text {**** }} \\
(4.65)\end{array}$ & & & $\begin{array}{c}2.972^{* * *} \\
(5.22)\end{array}$ & & & $\begin{array}{c}4.388^{* * *} \\
(3.87)\end{array}$ & & \\
\hline & & $\begin{array}{c}-0.757^{* * *} \\
(-2.89)\end{array}$ & & & $\begin{array}{l}-0.400^{*} \\
(-1.82)\end{array}$ & & & $\begin{array}{l}-0.400^{*} \\
(-1.82)\end{array}$ \\
\hline 0.228 & -0.223 & 1.027 & $0.390^{* * * *}$ & 0.567 & 0.0999 & $0.458^{* *}$ & -0.939 & 0.0999 \\
\hline$(1.45)$ & $(-0.70)$ & $(1.36)$ & $(2.70)$ & $(1.38)$ & $(0.13)$ & $(2.03)$ & $(-1.37)$ & $(0.13)$ \\
\hline 0.195 & $0.360^{* *}$ & -0.694 & $0.386^{* * *}$ & $0.231^{* * *}$ & $0.483^{* *}$ & $0.459^{* * *}$ & $0.363^{* * *}$ & $0.483^{* *}$ \\
\hline$(1.15)$ & $(2.21)$ & $(-1.09)$ & $(4.29)$ & $(2.67)$ & (1.99) & $(5.74)$ & $(3.55)$ & (1.99) \\
\hline 0.164 & -0.152 & 1.006 & $1.216^{* *}$ & 0.59 & 0.973 & $1.770^{* *}$ & 0.705 & 0.973 \\
\hline$(0.66)$ & $(-0.44)$ & $(1.50)$ & $(2.40)$ & $(1.02)$ & $(1.53)$ & $(2.38)$ & $(0.87)$ & $(1.53)$ \\
\hline 0.991 & 1.367 & -0.825 & 0.107 & 0.168 & -0.233 & -0.371 & 0.179 & -0.233 \\
\hline$(0.99)$ & $(1.26)$ & $(-0.66)$ & $(0.20)$ & $(0.29)$ & $(-0.49)$ & $(-0.36)$ & $(0.17)$ & $(-0.49)$ \\
\hline $0.383^{* *}$ & $0.487^{* * *}$ & $0.327^{*}$ & -0.0208 & 0.000442 & 0.0356 & 0.0575 & -0.304 & 0.0356 \\
\hline$(2.47)$ & $(2.86)$ & $(1.76)$ & $(-0.11)$ & 0.00 & $(0.13)$ & $(0.22)$ & $(-1.05)$ & $(0.13)$ \\
\hline 0.0227 & $0.150^{*}$ & 0.0559 & $0.274^{* * * *}$ & $0.240^{* *}$ & 0.168 & $0.383^{* * *}$ & $0.397^{* * *}$ & 0.168 \\
\hline$(0.31)$ & (1.77) & $(0.71)$ & $(3.48)$ & $(2.37)$ & (1.27) & (3.64) & (3.02) & $(1.27)$ \\
\hline 0.114 & 0.201 & 0.0242 & $0.424^{*}$ & $0.479^{*}$ & 0.415 & $0.998^{* *}$ & 0.534 & 0.415 \\
\hline$(0.49)$ & $(0.78)$ & $(0.07)$ & (1.77) & (1.85) & (1.02) & (2.38) & (1.16) & (1.02) \\
\hline-0.394 & -0.268 & -0.468 & -0.178 & -0.236 & -0.258 & $-0.453^{*}$ & -0.414 & -0.258 \\
\hline$(-1.06)$ & $(-0.69)$ & $(-1.09)$ & $(-1.14)$ & $(-1.28)$ & $(-1.26)$ & $(-1.69)$ & $(-1.39)$ & $(-1.26)$ \\
\hline$-0.257^{* * * *}$ & $-0.286^{* * * *}$ & -0.103 & $-0.207^{* * *}$ & $-0.146^{* * *}$ & $-0.310^{* * * *}$ & $-0.286^{* * * *}$ & $-0.293^{* * *}$ & $-0.310^{* * * *}$ \\
\hline$(-5.26)$ & $(-5.37)$ & $(-0.84)$ & $(-5.99)$ & $(-3.62)$ & $(-4.97)$ & $(-4.42)$ & $(-4.77)$ & $(-4.97)$ \\
\hline-0.0166 & -0.0115 & $-0.0284^{*}$ & 0.005 & -0.00282 & $0.0313^{* * *}$ & $-0.0294^{*}$ & -0.0234 & $0.0313^{* * *}$ \\
\hline$(-1.45)$ & $(-0.95)$ & $(-1.91)$ & $(0.65)$ & $(-0.29)$ & (2.69) & $(-1.80)$ & $(-1.23)$ & (2.69) \\
\hline 0.376 & 0.779 & $4.657^{*}$ & -1.214 & -0.159 & -0.746 & -1.689 & $-3.118^{* *}$ & -0.746 \\
\hline$(0.45)$ & $(0.88)$ & (1.79) & $(-1.54)$ & $(-0.16)$ & $(-0.57)$ & $(-1.35)$ & $(-2.13)$ & $(-0.57)$ \\
\hline 0.396 & 0.92 & -1.277 & 0.842 & $1.400^{*}$ & 0.569 & 0.349 & -0.091 & 0.569 \\
\hline$(0.61)$ & (1.49) & $(-1.06)$ & (1.22) & (1.67) & $(0.66)$ & $(0.31)$ & $(-0.06)$ & $(0.66)$ \\
\hline 1.034 & $1.877^{*}$ & -0.379 & 0.299 & 0.355 & 0.0278 & $1.398^{* *}$ & $2.118^{* * *}$ & 0.0278 \\
\hline (1.15) & $(1.75)$ & $(-0.21)$ & $(0.47)$ & $(0.46)$ & $(0.05)$ & (2.05) & $(2.25)$ & $(0.05)$ \\
\hline 0.168 & 0.00691 & 0.432 & -0.124 & -0.0152 & $-0.554^{* *}$ & $0.469^{*}$ & 0.393 & $-0.554^{* * *}$ \\
\hline
\end{tabular}


Table 2.4. Estimation Result of Subsamples by Different Regions

\begin{tabular}{|c|c|c|c|c|c|c|c|c|c|}
\hline & \multicolumn{3}{|c|}{ OECD Subsample } & \multicolumn{3}{|c|}{ Non-OECD Subsample } & \multicolumn{3}{|c|}{ BRIC Subsample } \\
\hline & (1) & (2) & (3) & (1) & (2) & (3) & (1) & (2) & (3) \\
\hline & $(1.02)$ & $(0.04)$ & $(1.62)$ & $(-0.87)$ & $(-0.09)$ & $(-2.11)$ & $(1.76)$ & $(1.19)$ & $(-2.11)$ \\
\hline \multicolumn{10}{|l|}{ Macroeconomic variables } \\
\hline \multirow[t]{2}{*}{ GDP growth $_{\mathrm{i}, \mathrm{t}}$} & -0.0703 & -0.166 & 0.0694 & $0.920^{* * * *}$ & $0.932^{* * * *}$ & $0.887^{* * * *}$ & 0.63 & 0.607 & $0.887^{*, * * *}$ \\
\hline & $(-0.38)$ & $(-0.90)$ & $(0.37)$ & $(4.60)$ & $(3.85)$ & $(3.59)$ & $(1.61)$ & $(1.33)$ & $(3.59)$ \\
\hline \multirow[t]{2}{*}{ Interest rate $\mathrm{e}_{\mathrm{i}, \mathrm{t}}$} & $-0.928^{*}$ & $-1.031^{*}$ & -0.946 & $-0.557^{* * *}$ & $-0.326^{*}$ & -0.253 & $-1.726^{* * *}$ & $-0.923^{* * *}$ & -0.253 \\
\hline & $(-1.72)$ & $(-1.71)$ & $(-1.49)$ & $(-3.45)$ & $(-1.79)$ & $(-1.62)$ & $(-4.06)$ & $(-2.23)$ & $(-1.62)$ \\
\hline \multicolumn{10}{|l|}{ Bank type variables } \\
\hline \multirow[t]{2}{*}{ Commercial dummy $* \delta_{\mathrm{t}-1}$} & 0.0705 & 0.0566 & 0.0253 & $-0.137^{* * *}$ & $-0.105^{*}$ & -0.102 & $-0.204^{* * *}$ & $-0.223^{* * *}$ & -0.102 \\
\hline & $(0.98)$ & $(0.76)$ & $(0.29)$ & $(-2.93)$ & $(-1.80)$ & $(-1.56)$ & $(-3.13)$ & $(-3.18)$ & $(-1.56)$ \\
\hline \multirow[t]{2}{*}{ Savings dummy $* \delta_{\mathrm{t}-1}$} & 0.0822 & 0.0646 & 0.052 & $-0.324^{* * *}$ & $-0.367^{*}$ & -0.135 & & & -0.135 \\
\hline & $(1.13)$ & $(0.96)$ & $(0.57)$ & $(-2.93)$ & $(-1.78)$ & $(-1.21)$ & & & $(-1.21)$ \\
\hline \multirow[t]{2}{*}{ Government dummy $* \delta_{\mathrm{t}-1}$} & -0.0142 & -0.0646 & 0.0554 & $0.128^{* * * *}$ & $0.125^{* * *}$ & $0.136^{* * *}$ & $0.175^{* * *}$ & $0.130^{* * *}$ & $0.136^{* * *}$ \\
\hline & $(-0.32)$ & $(-1.21)$ & $(0.87)$ & (4.89) & $(4.27)$ & $(4.24)$ & $(4.17)$ & $(2.89)$ & $(4.24)$ \\
\hline \multirow[t]{2}{*}{ Foreign dummy $* \delta_{\mathrm{t}-1}$} & -0.0553 & $-0.0835^{* * *}$ & 0.164 & -0.0757 & -0.107 & -0.00954 & & & -0.00954 \\
\hline & $(-0.98)$ & $(-2.82)$ & $(0.72)$ & $(-1.34)$ & $(-1.56)$ & $(-0.21)$ & & & $(-0.21)$ \\
\hline \multirow[t]{2}{*}{ Subsidiary dummy $* \delta_{\mathrm{t}-1}$} & 0.0241 & 0.0248 & 0.0408 & -0.00347 & 0.0513 & -0.00799 & 0.0629 & $0.109^{* *}$ & -0.00799 \\
\hline & $(0.92)$ & $(0.83)$ & $(1.56)$ & $(-0.12)$ & $(1.39)$ & $(-0.21)$ & (1.44) & $(2.36)$ & $(-0.21)$ \\
\hline$N$ & 5070 & 4028 & 3026 & 2305 & 1627 & 1512 & 919 & 733 & 1512 \\
\hline Adjusted-R2 & 0.116 & & & 0.227 & & & 0.349 & & \\
\hline Hansen J Statistic & & 1.97 & 18.06 & & 4.377 & 16.16 & & 0.0521 & 16.16 \\
\hline ( $p$-value) & & $(0.373)$ & $(0.114)$ & & $(0.112)$ & $(0.184)$ & & $(0.974)$ & $(0.184)$ \\
\hline Estimation Method & $\mathrm{FE}$ & IV & GMM & $\mathrm{FE}$ & IV & GMM & $\mathrm{FE}$ & IV & GMM \\
\hline
\end{tabular}

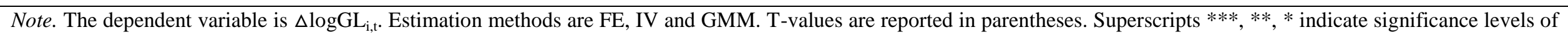
$1 \%, 5 \%$, and $10 \%$ respectively. 
Table 2.5. Estimation Result of Subsamples

\begin{tabular}{|c|c|c|c|c|c|c|c|c|c|c|}
\hline & (1) & (2) & (3) & (4) & (5) & (6) & (7) & (8) & (9) & (10) \\
\hline & $\begin{array}{c}\text { Bottom } \\
\text { TA }\end{array}$ & $\begin{array}{l}\text { Top } \\
\text { TA }\end{array}$ & $\begin{array}{c}\text { Bottom } \\
\text { Tier1 }\end{array}$ & $\begin{array}{c}\text { Top } \\
\text { Tier1 }\end{array}$ & $\begin{array}{c}\text { Bottom } \\
\text { Tier2 }\end{array}$ & $\begin{array}{c}\text { Top } \\
\text { Tier2 }\end{array}$ & $\begin{array}{c}\text { Bottom } \\
\text { TCD }\end{array}$ & $\begin{array}{c}\text { Top } \\
\text { TCD }\end{array}$ & $\begin{array}{l}\text { Bottom } \\
\text { DEP }\end{array}$ & $\begin{array}{l}\text { Top } \\
\text { DEP }\end{array}$ \\
\hline \multicolumn{11}{|c|}{ Bank specific variables } \\
\hline Intercept & $\begin{array}{c}3.424^{* * * *} \\
(4.93)\end{array}$ & $\begin{array}{c}2.535^{* * *} \\
(4.27)\end{array}$ & $\begin{array}{c}2.073^{* * *} \\
(3.79)\end{array}$ & $\begin{array}{c}3.302^{* * * *} \\
(4.63)\end{array}$ & $\begin{array}{c}3.110^{* * *} \\
(4.15)\end{array}$ & $\begin{array}{c}3.218^{* * *} \\
(4.92)\end{array}$ & $\begin{array}{c}4.036^{* * *} \\
(6.82)\end{array}$ & $\begin{array}{c}1.841^{* * *} \\
(2.85)\end{array}$ & $\begin{array}{c}2.948^{* * * *} \\
(3.92)\end{array}$ & $\begin{array}{c}3.396^{* * *} \\
(3.90)\end{array}$ \\
\hline TIER $1_{i, t-1}$ & $\begin{array}{c}0.438^{\text {**** }} \\
(2.96)\end{array}$ & $\begin{array}{l}0.116 \\
(0.54)\end{array}$ & $\begin{array}{c}1.482^{* * *} \\
(3.34)\end{array}$ & $\begin{array}{c}0.323^{* *} \\
(2.18)\end{array}$ & $\begin{array}{c}0.431^{* *} \\
(2.37)\end{array}$ & $\begin{array}{c}0.655^{* *} \\
(1.97)\end{array}$ & $\begin{array}{c}0.292^{* *} \\
(2.06)\end{array}$ & $\begin{array}{c}1.132^{* * *} \\
(3.58)\end{array}$ & $\begin{array}{c}0.388^{* *} \\
(2.17)\end{array}$ & $\begin{array}{l}0.131 \\
(0.71)\end{array}$ \\
\hline TIER $1_{\mathrm{i}, \mathrm{t}-1} * \delta_{\mathrm{t}-1}$ & $\begin{array}{c}0.381^{* * * *} \\
(4.42)\end{array}$ & $\begin{array}{l}0.161 \\
(0.72)\end{array}$ & $\begin{array}{l}-0.455 \\
(-1.04)\end{array}$ & $\begin{array}{c}0.340^{* * * *} \\
(3.02)\end{array}$ & $\begin{array}{c}0.394^{* * *} \\
(2.80)\end{array}$ & $\begin{array}{l}0.192 \\
(0.92)\end{array}$ & $\begin{array}{c}0.378^{* * * *} \\
(3.82)\end{array}$ & $\begin{array}{l}0.378 \\
(1.14)\end{array}$ & $\begin{array}{c}0.234^{* * * *} \\
(2.72)\end{array}$ & $\begin{array}{l}0.329 \\
(1.39)\end{array}$ \\
\hline TIER $2_{i, t-1}$ & $\begin{array}{l}1.282^{* *} \\
(2.18)\end{array}$ & $\begin{array}{l}0.126 \\
(0.50)\end{array}$ & $\begin{array}{l}1.056^{*} \\
(1.94)\end{array}$ & $\begin{array}{l}1.164^{* *} \\
(2.24)\end{array}$ & $\begin{array}{c}0.0855 \\
(0.08)\end{array}$ & $\begin{array}{l}-0.286 \\
(-0.54)\end{array}$ & $\begin{array}{l}0.574^{* *} \\
(2.21)\end{array}$ & $\begin{array}{l}0.259 \\
(0.57)\end{array}$ & $\begin{array}{l}0.726^{*} \\
(1.82)\end{array}$ & $\begin{array}{l}0.325 \\
(1.16)\end{array}$ \\
\hline TIER $2_{\mathrm{i}, \mathrm{t}-1} * \delta_{\mathrm{t}-1}$ & $\begin{array}{l}0.199 \\
(0.30)\end{array}$ & $\begin{array}{l}0.238 \\
(0.31)\end{array}$ & $\begin{array}{l}-0.454 \\
(-0.83)\end{array}$ & $\begin{array}{l}0.197 \\
(0.21)\end{array}$ & $\begin{array}{c}-0.0506 \\
(-0.03)\end{array}$ & $\begin{array}{l}-0.564 \\
(-0.82)\end{array}$ & $\begin{array}{l}0.645 \\
(0.75)\end{array}$ & $\begin{array}{l}0.432 \\
(0.78)\end{array}$ & $\begin{array}{l}0.718^{*} \\
(1.74)\end{array}$ & $\begin{array}{l}0.114 \\
(0.15)\end{array}$ \\
\hline $\mathrm{TCD}_{\mathrm{i}, \mathrm{t}-1}$ & $\begin{array}{c}0.18 \\
(1.15)\end{array}$ & $\begin{array}{l}0.237 \\
(1.59)\end{array}$ & $\begin{array}{c}0.346^{* *} \\
(2.16)\end{array}$ & $\begin{array}{c}0.0612 \\
(0.35)\end{array}$ & $\begin{array}{l}0.141 \\
(0.82)\end{array}$ & $\begin{array}{l}0.213 \\
(1.12)\end{array}$ & $\begin{array}{c}0.0637 \\
(0.41)\end{array}$ & $\begin{array}{c}0.458^{* *} \\
(2.51)\end{array}$ & $\begin{array}{l}0.215^{*} \\
(1.89)\end{array}$ & $\begin{array}{c}-0.0882 \\
(-0.33)\end{array}$ \\
\hline $\mathrm{TCD}_{\mathrm{i}, \mathrm{t}-1} * \delta_{\mathrm{t}-1}$ & $\begin{array}{c}0.0555 \\
(0.62)\end{array}$ & $\begin{array}{c}0.161^{* * *} \\
(3.09)\end{array}$ & $\begin{array}{c}0.0964^{* *} \\
(2.16)\end{array}$ & $\begin{array}{c}0.190^{* *} \\
(2.55)\end{array}$ & $\begin{array}{c}0.216^{* * *} \\
(2.67)\end{array}$ & $\begin{array}{l}0.0482 \\
(0.99)\end{array}$ & $\begin{array}{c}0.286^{* *} \\
(2.47)\end{array}$ & $\begin{array}{c}-0.0118 \\
(-0.07)\end{array}$ & $\begin{array}{c}0.0696 \\
(1.56)\end{array}$ & $\begin{array}{l}0.250^{* * *} \\
(2.06)\end{array}$ \\
\hline $\operatorname{DEP}_{i, t-1}$ & $\begin{array}{l}0.223 \\
(0.77)\end{array}$ & $\begin{array}{l}0.361^{*} \\
(1.74)\end{array}$ & $\begin{array}{c}0.0798 \\
(0.53)\end{array}$ & $\begin{array}{c}0.41 \\
(1.56)\end{array}$ & $\begin{array}{c}0.674^{* *} \\
(2.06)\end{array}$ & $\begin{array}{c}-0.12 \\
(-0.66)\end{array}$ & $\begin{array}{l}0.347^{*} \\
(1.80)\end{array}$ & $\begin{array}{l}0.581 \\
(1.24)\end{array}$ & $\begin{array}{l}-0.149 \\
(-0.26)\end{array}$ & $\begin{array}{c}0.34 \\
(1.31)\end{array}$ \\
\hline $\operatorname{DEP}_{\mathrm{i}, \mathrm{t}-1} * \delta_{\mathrm{t}-1}$ & $\begin{array}{l}-0.280^{*} \\
(-1.82)\end{array}$ & $\begin{array}{l}-0.331 \\
(-1.01)\end{array}$ & $\begin{array}{c}-0.0656 \\
(-0.57)\end{array}$ & $\begin{array}{l}-0.471 \\
(-1.39)\end{array}$ & $\begin{array}{l}-0.519 \\
(-1.34)\end{array}$ & $\begin{array}{c}-0.232^{* *} \\
(-2.03)\end{array}$ & $\begin{array}{c}-0.22 \\
(-1.23)\end{array}$ & $\begin{array}{c}-0.00951 \\
(-0.03)\end{array}$ & $\begin{array}{c}0.13 \\
(0.21)\end{array}$ & $\begin{array}{l}-0.316 \\
(-1.09)\end{array}$ \\
\hline $\mathrm{TA}_{\mathrm{i}, \mathrm{t}-1}$ & $\begin{array}{c}-0.278^{* * *} \\
(-5.41)\end{array}$ & $\begin{array}{c}-0.168^{* * *} \\
(-4.99)\end{array}$ & $\begin{array}{c}-0.158^{* * *} \\
(-4.85)\end{array}$ & $\begin{array}{c}-0.249^{* * *} \\
(-5.05)\end{array}$ & $\begin{array}{c}-0.239^{\text {*** }} \\
(-4.70)\end{array}$ & $\begin{array}{c}-0.222^{* * * *} \\
(-5.88)\end{array}$ & $\begin{array}{c}-0.287^{* * *} \\
(-7.43)\end{array}$ & $\begin{array}{c}-0.156^{\text {*** }} \\
(-4.03)\end{array}$ & $\begin{array}{c}-0.218^{* * *} \\
(-4.29)\end{array}$ & $\begin{array}{c}-0.227^{\text {*** }} \\
(-4.49)\end{array}$ \\
\hline $\mathrm{TA}_{\mathrm{i}, \mathrm{t}-1} * \delta_{\mathrm{t}-1}$ & $\begin{array}{c}0.00395 \\
(0.26)\end{array}$ & $\begin{array}{c}0.00466 \\
(0.68)\end{array}$ & $\begin{array}{c}0.00277 \\
(0.62)\end{array}$ & $\begin{array}{c}-0.00221 \\
(-0.22)\end{array}$ & $\begin{array}{c}-0.00636 \\
(-0.82)\end{array}$ & $\begin{array}{c}0.0091 \\
(1.25)\end{array}$ & $\begin{array}{c}-0.00438 \\
(-0.61)\end{array}$ & $\begin{array}{c}0.0125^{* *} \\
(2.40)\end{array}$ & $\begin{array}{c}-0.00302 \\
(-0.73)\end{array}$ & $\begin{array}{c}0.00871 \\
(0.72)\end{array}$ \\
\hline $\operatorname{LLP}_{\mathrm{i}, \mathrm{t}-1}$ & $\begin{array}{l}-0.955 \\
(-1.12)\end{array}$ & $\begin{array}{c}-1.324^{* *} \\
(-2.18)\end{array}$ & $\begin{array}{l}-0.401 \\
(-0.65)\end{array}$ & $\begin{array}{l}-1.144 \\
(-1.28)\end{array}$ & $\begin{array}{l}-1.043 \\
(-0.97)\end{array}$ & $\begin{array}{c}-1.998^{* * *} \\
(-2.74)\end{array}$ & $\begin{array}{l}-1.157 \\
(-1.39)\end{array}$ & $\begin{array}{c}-1.640^{\text {*** }} \\
(-3.17)\end{array}$ & $\begin{array}{l}-0.452 \\
(-0.62)\end{array}$ & $\begin{array}{l}-0.643 \\
(-0.79)\end{array}$ \\
\hline $\mathrm{FA}_{\mathrm{i}, \mathrm{t}-1}$ & $\begin{array}{l}1.019 \\
(1.12)\end{array}$ & $\begin{array}{l}0.213 \\
(0.47)\end{array}$ & $\begin{array}{l}0.128 \\
(0.18)\end{array}$ & $\begin{array}{l}0.838 \\
(1.07)\end{array}$ & $\begin{array}{l}-0.491 \\
(-0.53)\end{array}$ & $\begin{array}{l}1.218 \\
(1.42)\end{array}$ & $\begin{array}{l}1.370^{* *} \\
(2.00)\end{array}$ & $\begin{array}{l}-0.413 \\
(-0.38)\end{array}$ & $\begin{array}{l}-1.744 \\
(-1.64)\end{array}$ & $\begin{array}{l}1.134 \\
(1.49)\end{array}$ \\
\hline $\mathrm{ROA}_{\mathrm{i}, \mathrm{t}-1}$ & $\begin{array}{c}0.00903 \\
(0.01)\end{array}$ & $\begin{array}{l}0.832 \\
(1.38)\end{array}$ & $\begin{array}{c}0.98 \\
(1.15)\end{array}$ & $\begin{array}{l}0.155 \\
(0.21)\end{array}$ & $\begin{array}{l}0.443 \\
(0.56)\end{array}$ & $\begin{array}{l}-0.931 \\
(-1.39)\end{array}$ & $\begin{array}{l}0.393 \\
(0.59)\end{array}$ & $\begin{array}{l}-0.708 \\
(-1.00)\end{array}$ & $\begin{array}{l}-1.401^{*} \\
(-1.74)\end{array}$ & $\begin{array}{l}1.135 \\
(1.47)\end{array}$ \\
\hline
\end{tabular}


Table 2.5. Estimation Result of Subsamples

$\delta_{\mathrm{t}-1}$

\begin{tabular}{|c|c|c|c|c|c|c|c|c|c|c|}
$(1)$ & $(2)$ & $(3)$ & $(4)$ & $(5)$ & $(6)$ & $(7)$ & $(8)$ & $(10)$ \\
\hline Bottom & Top & Bottom & Top & Bottom & Top & Bottom & Top & Bottom & Top \\
TA & TA & Tier1 & Tier1 & Tier2 & Tier2 & TCD & TCD & DEP \\
\hline-0.0909 & -0.0966 & 0.018 & -0.0319 & -0.0251 & -0.0742 & -0.101 & -0.152 & 0.0188 \\
$(-0.41)$ & $(-0.83)$ & $(0.21)$ & $(-0.22)$ & $(-0.25)$ & $(-0.57)$ & $(-0.86)$ & $(-0.78)$ & $(0.36)$ & $(-1.01)$
\end{tabular}

Macroeconomic variables

\begin{tabular}{|c|c|c|c|c|c|c|c|c|c|c|}
\hline GDP growth $_{\mathrm{i}, \mathrm{t}}$ & $\begin{array}{c}0.888^{\text {**** }} \\
(3.75)\end{array}$ & $\begin{array}{c}0.720^{* * *} \\
(3.14)\end{array}$ & $\begin{array}{c}0.618^{\text {**** }} \\
(3.97)\end{array}$ & $\begin{array}{c}0.819^{\text {*** }} \\
(3.19)\end{array}$ & $\begin{array}{c}0.655^{\text {*** }} \\
(2.86)\end{array}$ & $\begin{array}{c}1.008^{* * *} \\
(4.80)\end{array}$ & $\begin{array}{c}0.548^{* *} \\
(2.15)\end{array}$ & $\begin{array}{c}0.754^{\text {*** }} \\
(3.83)\end{array}$ & $\begin{array}{l}0.273 \\
(1.56)\end{array}$ & $\begin{array}{c}0.910^{\text {*** }} \\
(2.97)\end{array}$ \\
\hline Interest rate $e_{i, t}$ & $\begin{array}{c}-0.928^{* * *} \\
(-3.66)\end{array}$ & $\begin{array}{c}-0.411^{* * *} \\
(-2.66)\end{array}$ & $\begin{array}{c}-0.260^{* *} \\
(-1.99)\end{array}$ & $\begin{array}{c}-0.880^{* * *} \\
(-3.89)\end{array}$ & $\begin{array}{c}-0.774^{* * *} \\
(-3.51)\end{array}$ & $\begin{array}{c}-0.385^{\text {**** }} \\
(-2.79)\end{array}$ & $\begin{array}{c}-1.252^{* * *} \\
(-5.55)\end{array}$ & $\begin{array}{l}-0.212 \\
(-1.02)\end{array}$ & $\begin{array}{l}-0.309^{*} \\
(-1.78)\end{array}$ & $\begin{array}{c}-0.722^{* * *} \\
(-3.49)\end{array}$ \\
\hline \multicolumn{11}{|c|}{ Industry structure variables } \\
\hline $\mathrm{HHI}_{\mathrm{i}, \mathrm{t}-1}$ & $\begin{array}{l}-1.057^{*} \\
(-1.69)\end{array}$ & $\begin{array}{l}-0.424 \\
(-1.06)\end{array}$ & $\begin{array}{c}0.0223 \\
(0.08)\end{array}$ & $\begin{array}{l}-1.391^{*} \\
(-1.81)\end{array}$ & $\begin{array}{l}0.188 \\
(0.51)\end{array}$ & $\begin{array}{c}-0.769^{* *} \\
(-2.01)\end{array}$ & $\begin{array}{c}-1.265^{* * *} \\
(-2.85)\end{array}$ & $\begin{array}{l}-0.485 \\
(-0.66)\end{array}$ & $\begin{array}{c}-0.41 \\
(-0.67)\end{array}$ & $\begin{array}{l}-0.614^{*} \\
(-1.87)\end{array}$ \\
\hline $\mathrm{HHI}_{\mathrm{i}, \mathrm{t}-1} * \delta_{\mathrm{t}-1}$ & $\begin{array}{l}0.801^{* *} \\
(2.27)\end{array}$ & $\begin{array}{l}-0.163 \\
(-0.62)\end{array}$ & $\begin{array}{c}-0.0748 \\
(-0.28)\end{array}$ & $\begin{array}{l}0.317 \\
(0.95)\end{array}$ & $\begin{array}{l}0.268 \\
(0.78)\end{array}$ & $\begin{array}{l}0.382 \\
(1.55)\end{array}$ & $\begin{array}{l}0.690^{*} \\
(1.78)\end{array}$ & $\begin{array}{c}-0.06 \\
(-0.19)\end{array}$ & $\begin{array}{l}-0.322 \\
(-1.22)\end{array}$ & $\begin{array}{l}0.433 \\
(1.37)\end{array}$ \\
\hline COMPTIER $1_{\mathrm{i}, \mathrm{t}-1}$ & $\begin{array}{l}0.105 \\
(0.68)\end{array}$ & $\begin{array}{c}0.363^{* *} \\
(2.44)\end{array}$ & $\begin{array}{l}0.216^{* *} \\
(2.17)\end{array}$ & $\begin{array}{l}0.233 \\
(1.47)\end{array}$ & $\begin{array}{l}0.192 \\
(1.26)\end{array}$ & $\begin{array}{c}0.330^{* *} \\
(2.44)\end{array}$ & $\begin{array}{l}0.083 \\
(0.57)\end{array}$ & $\begin{array}{l}0.262^{*} \\
(1.83)\end{array}$ & $\begin{array}{c}0.303^{* * *} \\
(3.25)\end{array}$ & $\begin{array}{c}0.00621 \\
(0.02)\end{array}$ \\
\hline COMPTIER $1_{\mathrm{i}, \mathrm{t}-1} * \delta_{\mathrm{t}-1}$ & $\begin{array}{l}-0.285 \\
(-0.81)\end{array}$ & $\begin{array}{c}-0.539^{* * *} \\
(-3.25)\end{array}$ & $\begin{array}{c}-0.461^{* * *} \\
(-3.84)\end{array}$ & $\begin{array}{l}-0.405 \\
(-1.45)\end{array}$ & $\begin{array}{l}-0.287 \\
(-1.27)\end{array}$ & $\begin{array}{c}-0.541^{* * *} \\
(-3.26)\end{array}$ & $\begin{array}{l}-0.273 \\
(-0.92)\end{array}$ & $\begin{array}{c}-0.414^{* *} \\
(-2.51)\end{array}$ & $\begin{array}{c}-0.379^{* * *} \\
(-3.03)\end{array}$ & $\begin{array}{c}0.00713 \\
(0.02)\end{array}$ \\
\hline$N$ & 3728 & 3646 & 3505 & 3869 & 3875 & 3499 & 4465 & 2909 & 4356 & 3018 \\
\hline Adjusted-R2 & 0.262 & 0.157 & 0.137 & 0.177 & 0.170 & 0.240 & 0.222 & 0.121 & 0.146 & 0.169 \\
\hline Estimation Method & FE & $\mathrm{FE}$ & $\mathrm{FE}$ & $\mathrm{FE}$ & FE & $\mathrm{FE}$ & $\mathrm{FE}$ & FE & FE & $\mathrm{FE}$ \\
\hline
\end{tabular}

$10 \%$ respectively. In "Top" and "Bottom" in each column are observations "above" and "below" the mean of each variable in each year respectively. 


\subsection{Conclusion}

In this study, we examine the relationship between loan growth and bank capital structure. We analyze the impact of the type of bank funding on bank lending behavior in the global financial crisis. We distinguish between tier 1 and tier 2 capital and customer and interbank deposits as bank funding sources. We combine unbalanced panel data using annual balance sheet bank data between 2000 and 2010 with several variables discerning the macroeconomic environment, organizational and ownership structure, regulatory environment, and government support.

We find a significant and positive effect of the tier 1 capital ratio on bank loan growth during the global financial crisis. The effect seems to be more pronounced for small banks and for banks in non-OECD and BRIC countries. Customer deposits also positively affected bank lending during the global financial crisis. Furthermore, we find some (but weak) evidence that the tier 2 capital ratio and interbank deposits positively affect loan growth in normal times and that interbank deposits negatively affected bank lending during the global financial crisis.

Our evidence highlights a sharp contrast on the impact of different funding sources on bank lending during the global financial crisis. Whereas tier 1 capital and customer deposits acted as a stable source of funding during the global financial crisis, tier 2 capital and interbank deposits spur bank lending during normal times, but did not do so during the global financial crisis.

We also find that during normal times a bank lends more if the tier 1 capital ratio of competing banks is high. This relationship reversed during the global financial crisis. That is, during the global financial crisis a bank lent more if the tier 1 capital ratio of competing banks was low. The effect is present only for the subsample of large banks but not for small banks. This may indicate that large banks gained a competitive advantage against weakly capitalized competitors especially during the global financial crisis but not during normal times.

We also find that government ownership helped banks better sustain credit growth during the global financial crisis. This effect was statistically significant only in non-OECD and BRIC countries, but not in OECD countries. 


\section{COMPETITION IN THE CLEARING AND SETTLEMENT INDUSTRY ${ }^{40}$}

\subsection{Overview}

This chapter empirically analyzes the competitive landscape within the clearing and settlement industry. Using the panel data of 49 clearing and settlement institutions from 24 countries, we confirm that clearing and settlement institutions operate under the monopoly equilibrium. We show that competition increases with the institutional size, mergers, and with technological development. Additionally, we find some evidence that competition in clearing and settlement is higher during the global financial crisis compared to normal times. We also show that competition between clearing and settlement institutions is higher in the U.S. than in Europe.

\subsection{Introduction}

Amid increasingly globalized financial markets, clearing and settlement institutions need to operate in an international and fast-changing environment. To reach international scale, some of the clearing and settlement institutions went through a massive consolidation process. For example, Belgium-based central securities depository (CSD) Euroclear became the largest international central securities depository in the world through a series of acquisitions (of French CSD Sicovam in 2001, the Dutch CSD Necigef and the UK CSD CrestCo in 2002, the Belgian CSD CIK in 2007, the CSD of Finland APK and Sweden VPC AB in 2008). Although consolidation brought internationalization and rapid expansion of cross-border clearing and settlement activities, it might also have negatively affected the competitive landscape. Due to the antitrust concerns, the European Commission prohibited the proposed merger between Deutsche Börse AG and NYSE Euronext in 2012. According to the European Commission, the merged company would have obtained near-monopolistic power in trading and clearing of European exchange-traded derivatives. ${ }^{41}$ Competition in clearing and settlement is therefore becoming a foremost issue.

We use unbalanced annual financial data of 49 clearing and settlement institutions from 24 countries between 1989 and 2012 to perform a comprehensive panel-based analysis of competition between clearing and settlement institutions across the European and the U.S. market. We employ the Panzar-Rosse model (Panzar and Rosse, 1982, 1987) (hereafter 'PR model') to estimate the competitive indicator ' $H$-statistic' that shows whether clearing and settlement institutions operate under a monopoly, monopolistic competition, or perfect competition. We also compute the Lerner index of monopoly power of clearing and settlement institutions (following Coccorese, 2009; Koetter et al., 2012) and the Boone indicator (Boone, 2001, 2008). We investigate several factors that affect competition in the clearing and settlement

\footnotetext{
40 This chapter is co-authored with Matej Marinč. We would like to thank Iftekhar Hasan, Marko Košak, and Igor Lončarski, and the participants at the 2nd EBR Conference in Ljubljana and at the 9th EBES Conference in Rome for their valuable comments and suggestions.

41 See

http://europa.eu/rapid/pressReleasesAction.do?reference=IP/12/94\&format=HTML\&aged=0\&language=EN\&guiLa $\underline{\text { nguage }=\text { en }}$.
} 
industry, including the role of mergers and acquisitions, size, institutional structure, technological development, and the global financial crisis.

We confirm that clearing and settlement institutions operate under the monopoly equilibrium. We show that competition increases with the institutional size, mergers, and with technological development. Additionally, we find some evidence that competition in clearing and settlement is higher during the global financial crisis compared to normal times. We also show that competition between clearing and settlement institutions is higher in the U.S. than in Europe.

To our knowledge, we are the first to analyze competition in the clearing and settlement industry using the PR model, Lerner index, and Boone indicator, which have frequently been applied in the banking literature (Angelini and Cetorelli, 2003; Bikker, Spierdijk, and Finnie, 2006; 2007). Previous studies provide empirical evidence on the existence of economies of scale, relative efficiency, and technological development in clearing and settlement and in stock exchange markets (Hasan and Malkamäki, 2001; Schmiedel, 2001; Hasan et al., 2003; Hasan and Schmiedel, 2004; Hasan, Schmiedel, and Song, 2012a; Van Cayseele and Wuyts, 2007). ${ }^{42}$ We analyze the competitive environment and the factors that affect competition in the clearing and settlement industry.

The remainder of the chapter is organized as follows: Section 3.3 discusses the role of clearing and settlement institutions, reviews the literature, and builds hypotheses. Section 3.4 describes the methodology. Section 3.5 provides descriptive statistics and concentration measures. In Section 3.6, we analyze competition and factors that affect competition in clearing and settlement using the PR model. Section 3.7 provides evidence from the Lerner index and Section 3.8 focuses on the Boone indicator. Section 3.9 concludes this chapter.

\subsection{Literature Review}

\subsubsection{Clearing and settlement institutions in Europe and in the U.S.}

Clearing and settlement services facilitate well-functioning of capital markets by lowering transaction costs that an investor faces when completing a trade (Giddy, et al., 1996; Schaper, 2008). When a security is transacted in the securities market, the trade has to be cleared and settled only then the transaction can be completed. Clearing is the process in which the buyer of a security and its seller establish the respective obligations. Settlement implies the transfer of money from the buyer to the seller, and simultaneous delivery of the securities from the seller to the buyer. The clearing and settlement institutions guarantee that these transactions are performed safely and efficiently. Countries generally have highly centralized and integrated clearing and settlement industry.

Three types of organizations are providing clearing and settlement services: domestic central

\footnotetext{
${ }^{42}$ Previous studies show that economies of scale, technological development, cost and revenue efficiency, and mergers and acquisition affect the performance of stock exchanges and clearing and settlement industry (Hasan and Malkamäki (2001); Schmiedel, Malkamäki, and Tarkka (2006); Nielsson (2009)).
} 
securities depositories (CSDs), international central securities depositories (ICSDs), and custodians. CSDs are engaged in the settlement of securities, traded on their respective domestic markets, and are frequently part of the exchange in their domestic country. CSDs enable processing and settlement of securities transactions by book entry. They provide custodial services (e.g., the administration of corporate actions and redemptions), and play an active role in ensuring the integrity of securities' issues. Historically, ICSDs' main function was to settle Eurobond trades. They are now active in clearing and settlements across different international markets, and currency areas. ICSDs typically also provide a wide range of ancillary services, such as securities lending, voluntary corporate actions, tax services, proxy voting, and collateral management. ${ }^{43}$ Custodians are large investment banks that provide securities custody services to its customers. We focus on CSDs and ICSDs in our analysis. ${ }^{44}$

Clearing and settlement infrastructures differ across the main capital markets. We focus on the European and the U.S. market. In the U.S. market, the Depository Trust Company, Fixed Income Clearing Corporation, and National Securities Clearing Corporation operate under the Depository Trust \& Clearing Corporation, and they clear and settle almost all the securities transactions (more than U.S. \$1.6 quadrillion in transactions every year) ${ }^{45}$

Clearing and settlement infrastructure is less integrated in Europe than in the U.S. Around 40 CSDs operate in the domestically-oriented and fragmented European market. In addition to CSDs, Clearstream International and Euroclear Group act as ICSDs and provide services in many different markets to domestic or cross-border investors. Clearstream International clears and settles securities transactions in over 110 countries and its global network extends across 50 markets. Clearstream International uses the services of a local agent, which can be either a local CSD or a financial institution in the local market. Similar to Clearstream International, Euroclear Group focuses on clearing and settlement of international trade securities. It operates in more than 90 countries (Giovannini Group, 2002). The costs of cross-border clearing and settlement services are significantly higher than in the domestic market (De Carvalho, 2004; Giovannini Group, 2002; Schmiedel and Schönenberger, 2005).

Several initiatives are directed towards establishing more integrated European clearing and settlement. In 2012, the European Commission issued a proposal for the regulation of CSDs to strengthen the legal framework for uniform financial market infrastructure in the EU and provide the legal basis for the introduction of TARGET2-Securities (T2S) infrastructure. ${ }^{46}$ T2S infrastructure aims at overcoming fragmentation across national settlement systems within the EU by offering a single IT platform for settlement across borders, national CSDs, and currencies. According to the European Central Bank $(2007,2008)$, the average costs for securities settlement

\footnotetext{
43 European Central Bank, "CSD Ancillary Services”, 28 October 2011. See https://www.ecb.europa.eu/paym/t2s/progress/pdf/hsg/mtg4/2011-11-07-csd-ancillary-services-status.pdf??b7560d6 3bcb62dd376a6c405e4133e3c.

${ }^{44}$ Clearing and settlement presents only a fraction of business of custodian banks. Therefore, incorporating accounting figures of custodian banks in our empirical analysis would distort our measures of competition in clearing and settlement.

45 See also http://www.dtcc.com/ /media/Files/Downloads/About/DTCC_Capabilities.ashx.

46 See http://europa.eu/rapid/press-release_IP-12-221_en.htm?locale=en.
} 
through $\mathrm{T} 2 \mathrm{~S}$ infrastructure could be reduced to $€ 0.28$ per transaction; however, the participation of all relevant CSDs is essential for the success of T2S infrastructure. If the participation in T2S infrastructure is voluntary, the low number of transactions could raise the costs per transaction (Schaper, 2008). The Eurosystem invited all CSDs in Europe to outsource their settlement services to T2S. By 2012, 22 CSDs have signed a legal agreement ('Framework Agreement') with the Eurosystem, including almost all CSDs in the euro area (Mercier and Sauer, 2013).

Differences in integration across the U.S. and Europe may affect the level of competition in clearing and settlement.

\subsubsection{Industry structure in clearing and settlement services}

The extant literature on industry structure in clearing and settlement provides some evidence of scale economies, mergers, and the type of competition within clearing and settlement. ${ }^{47}$

First, empirical research identifies economies of scale in the clearing and settlement industry in the U.S. and Europe. Demsetz (1968) documents the existence of economies of scale in the New York Stock Exchange. Hancock, Humphrey, and Wilcox (1999) provide evidence for economies of scale in Fedwire electronic funds transfer operation. Adams, Bauer, and Sickles (2004) find significant economies of scale and scope in the Federal Reserve's payment processing services.

Van Cayseele and Wuyts (2007) find that economies of scale exist in European clearing and settlement; however, they are exhausted far below the size of the entire European market. Schmiedel et al. (2006) show that the level of economies of scale varies by the size of a clearing and settlement institution. Smaller settlement service providers have a high potential to further exploit economies of scale. However, larger institutions are already becoming more and more cost effective. ${ }^{48}$ Hasan and Malkamäki (2001) provide evidence for significant economies of scale and scope among European stock exchanges. Schmiedel (2001) finds that the size of stock exchanges, index of market concentration, quality, structural reorganizations of exchange governance, diversification in trading service activities, and adoption of automated trading systems have a significant impact on how efficiently trading services are provided in Europe. Hasan, Schmiedel, and Song (2012) find that mergers among stock exchanges improve performace in the short run and in the long run. Mergers bring value especially in the case of horizontal and cross-border integration.

Second, several authors weigh the benefits of mergers within the trading infrastructure and clearing and settlement industry with potential anti-competitive concerns. Tapking and Yang

\footnotetext{
47 Table C.1 in Appendix C summarizes the previous empirical studies on clearing and settlement institutions.

48 Developments in the payment processing industry might indicate the future of clearing and settlement services in the EU. Beijnen and Bolt (2009) confirm that significant economies of scale are present within eight European payment processors. They argue that a single European payments area will facilitate consolidation among European payment processors, which will further exploit payment economies of scale. Bolt and Humphrey (2007) see substantial cost efficiency gains in cross border consolidation of payment processing in the European market. Developed payment infrastructure is also important for the performance of the banking system. Hasan, Schmiedel, and Song (2012) show that bank performance is higher in countries with more developed retail payment service markets.
} 
(2006) show that vertical integration of domestic service providers (integration of trading infrastructure with the clearing and settlement infrastructure) may be desirable if domestic investors are not inclined to invest in foreign securities (see also Pirrong, 2007). However, horizontal integration of CSDs improves welfare if investors want to invest in foreign securities. Köppl and Monnet (2007) argue that vertical silos (between CSDs and exchanges) can prevent efficiency gains from horizontal consolidation between CSDs.

Rochet (2005) analyzes whether it is optimal for a CSD to compete with or be allowed to merger vertically with custodian banks. He finds that the welfare effect of a vertical integration depends on the trade-off between efficiency gains and lower competition at the custodian level (see also Kauko, 2007). Cherbonnier and Rochet (2010) conclude that vertical integration spurs the need for regulation of access pricing and this introduces new inefficiencies, due to the incentives of the ICSD to hide cost information. Holthausen and Tapking (2007) also analyze competition between CSDs and the agent banks. They find that a CSD raises its rival's cost to increase its monopoly power at the custodian level.

Third, the literature on the competition in clearing and settlement is rather scarce. Van Cayseele (2004) argues that contestable quasi-monopolies might be the efficient industry configuration in European clearing and settlement. In the contestable quasi-monopolies outcome, a few (international) CSDs would grow sufficiently large to exploit most of the economies of scale, but would still compete against each other.

Milne (2007a) argues that several services of clearing and settlement institutions (e.g., the book entry function and the transmission of corporate actions) are characterized as a natural monopoly, at the issuer level. Milne stresses that these core functions should be kept as a monopoly to exploit economies of scale. However, competition should increase in all other clearing and settlement services, at domestic but even more so at the European level. An abuse of the CSDs' monopoly position can be contained by regulation on terms and pricing of access (see also Juranek and Walz, 2010).

Serifsoy and Weiß (2007) find that market forces coupled with regulatory framework can provide for contestable monopolies outcome that ensure a high degree of static, dynamic, and systemic efficiency.

\subsubsection{Formation of hypotheses}

During the financial crises, clearing and settlement institutions face severe pressure and potentially higher competition from other financial institutions (e.g., custodian banks). Bernanke (1990) points out that the clearing and settlement services faced severe problems during the 1987 stock market crash. Lloyd Blankfein, CEO and Chairman of Goldman Sachs Group Inc., said "I agree that clearinghouses make things less risky for the regular crisis, but in an extreme crisis that could affect the clearinghouse itself." ${ }^{49}$ Our first hypothesis is therefore the following:

\footnotetext{
${ }^{49}$ See Elena Logutenkova and Fabio Benedetti-Valentini, "Blankfein Says Clearinghouses May Increase Risks in Crises," Bloomberg Businessweek, September 29, 2010,
} 
Hypothesis 3.1: Competition in clearing and settlement increased during the global financial crisis.

Lannoo and Levin (2002) examine the structure of the settlement and depository industry in the EU and account for the difference between CSDs and ICSDs. They find that ICSDs incur higher operating costs than CSDs because of more complex back-office systems and higher costs in cross-border settlement. Despite higher costs of cross-border transactions, ICSDs are confronted with direct competition from other ICSDs or from local CSDs. Therefore, we test the following hypothesis.

Hypothesis 3.2: ICSDs are exposed to a higher level of competition than CSDs.

The empirical analysis of the relation between the competition and the size of clearing and settlement institutions is scarce. Borrowing from the banking literature, some studies confirm a positive relation between the market power and size (see e.g. Bikker and Bos, 2005; Bikker, et al., 2006). Alternatively, smaller institutions operate primarily on local markets with weaker competition whereas larger institutions primarily operate on international level with generally a higher level competition. Larger institutions also engage in multimarket contact, which increases competition (see Mester, 1987). Several empirical studies based on the PR model confirm the negative relationship between asset size and market power (Bikker, 2004; see Hempell, 2002). If competition in clearing and settlement is limited on domestic markets but fierce on the international level, we can hypothesize the following:

Hypothesis 3.3: Larger size of clearing and settlement institutions is associated with higher competition.

Theoretical studies show that vertical and horizontal mergers might improve welfare in clearing and settlement. Consolidation in the clearing and settlement systems through vertical and horizontal mergers and alliances is modifying the European landscape. ICSDs are increasingly acquiring domestic CSDs (see, for example, an expansion strategy of Clearstream International and Euroclear System through mergers and acquisitions). The intention of these mergers is to reduce costs and boost efficiency but at the same time, international mergers may open up previously closed domestic clearing and settlement markets. ${ }^{50}$

Hypothesis 3.4: Mergers between CSDs are associated with higher competition.

The developments of information technology might also lead to substantial transformation of the clearing and settlement industry. Developments in information technology generally increase efficiency in the financial industry, but may also increase transaction nature of financial services, which is associated with higher competition (see Boot, 2014; Marinč, 2013).

http://www.bloomberg.com/news/2010-09-29/blankfein-says-using-clearing-houses-could-increase-risks.html

50 We focus on horizontal mergers between CSDs rather than on vertical mergers between CSDs and custodian banks. 
Hasan et al. (2003) find that the investments in standardization and new technologies increase the productivity of stock exchanges. Knieps (2006) argues that the implementation of new systems and further development in settlement technology improves cost effectiveness in the post-trade markets. Developments in information and communication technology promote integration of financial markets in the euro area (see e.g. Hasan and Malkamäki, 2001; Schmiedel, et al., 2006), reduce the importance of location for efficiency of transactions, and foster a single market especially if regulatory barriers are also removed (see Gehrig and Stenbacka, 2007). Information technology serves as a competitive factor in the post-trading industry (Schaper and Chlistalla, 2010).

Hypothesis 3.5: Technological development increases competition between clearing and settlement institutions.

Competition in clearing and settlement might differ across markets. Giovannini Group (2002) finds substantial barriers to European financial market integration in cross-border clearing and settlements. Lannoo and Levin (2002) confirm that the operating cost of securities settlement is higher in the EU than in the U.S. NERA Economic Consulting (2004) finds that the main reasons for higher clearing and settlement costs in Europe, compared to the U.S., are lower volume, several legal, regulatory, and technical barriers to non-domestic clearing and settlement in Europe, and differences in market structure. European clearing and settlement might still be substantially fragmented and this might hamper the level of competition compared to the U.S.

Hypothesis 3.6: Competition between clearing and settlement institutions is higher in the U.S. than in Europe.

\subsection{Methodological Basis to Measuring Competition}

We now provide the methodological basis for the competition analysis. That is, we discuss concentration indexes, Panzar Rosse $H$-statistic, Lerner index, and Boone indicator.

\subsubsection{Concentration indicators}

Previous empirical studies have measured competition through structural and non-structural approaches. The structural approach relies on structure-conduct-performance (SCP) paradigm that links concentration, competition, and firm performance. That is, SCP assumes that the market structure, reflected in the level of concentration in the market, affects firm behavior, which in turn determines firm performance (Bain, 1951; Mason, 1939). The two most commonly used concentration indices in empirical SCP studies are concentration ratio (CR) and Herfindahl-Hirschman index (HHI). CR measures the total market share of a given number of firms with the largest market shares. HHI is the sum of the squares of the market shares of the firms in the market. The problem with structural measures is that concentration does not necessarily determine competitive behavior of firms in the market. For example, in a contestable monopoly, a monopolistic firm may set competitive prices under the threat of new entry. 
A non-structural approach to measuring competition is the new empirical industrial organization (NEIO) approach. Unlike the SCP paradigm that tries to determine competition from the market structure in a given industry, the NEIO models directly analyze firm conduct to detect the market power of firms.

\subsubsection{Panzar-Rosse model}

The NEIO models can rely on a comparative statics analysis as in the PR model. The PR model identifies the market power by using the index $H$-statistic. $H$-statistic is calculated as the sum of revenue elasticities with respect to input prices. It measures how much a change in factor prices affects in the firm's equilibrium revenue.

The PR model was widely applied to measure competition in the banking industry (for the U.S. banking industry, see Shaffer (1982); for the Canadian banking industry, see Nathan and Neave (1989) and Shaffer (1993)). ${ }^{51}$ Vesala (1995) investigates how deregulation in the 1980s affected the competition among Finnish banks. Coccorese (2004; 2009) analyzes the competitive conditions in the Italian banking industry. Hempell (2002) analyzes competitive behavior of the German banking industry. Matthews et al. (2007) and Maudos and Solís (2011) employ the PR model and Lerner index to analyze competition in the British banking industry and in the Mexican banking industry, respectively. These findings mostly indicate that banks operate under monopolistic competition. ${ }^{52}$

The PR model is robust to the imprecisions in extent of the market (Shaffer, 2004a). That is, because the empirical specification requires only firm-level data, market definition is not needed in the revenue equation. This feature makes it especially suitable for the clearing and settlement institutions, which can easily span across countries and markets and face some competition from other financial institutions.

According to Bikker and Haaf (2002), the PR model assumes a log-log marginal cost function (MC) of the following form:

$\ln \mathrm{MC}=\alpha_{0}+\alpha_{1} \ln \mathrm{OUT}+\sum_{\mathrm{i}=1}^{\mathrm{m}} \beta_{\mathrm{i}} \ln \mathrm{FIP}_{\mathrm{i}}+\sum_{\mathrm{j}=1}^{\mathrm{p}} \gamma_{\mathrm{j}} \ln \mathrm{EX}_{\mathrm{COST}_{\mathrm{j}}}$

\footnotetext{
51 In Appendix C, Table C.2 summarizes the empirical studies that use Panzar-Rosse Model and the Lerner index to measure competition in the banking industry.

52 Several studies analyze competition in banking industry across countries. De Bandt and Davis (2000) provide evidence that the behavior of large banks in the EMU was less competitive compared to the banks in the U.S. Competition appears to be lower among small banks, especially in France and Germany. Bikker and Haaf (2002) work on a study of 23 industrialized countries and conclude that in local markets competition is weaker than in international markets. Gelos and Roldós (2004) focus on eight emerging markets during the 1990s and argue that lower entry barriers mitigated a decline in competition driven by consolidation. Claessens and Laeven (2004) analyze competition across 50 banking systems and argue that higher competition is associated with lower restrictions to bank entry and to bank activities. Schaeck, Cihak, and Wolfe (2009) provide evidence that more competitive banking systems are less likely to undergo a systemic crisis. Liu, Molyneux, and Wilson (2013a) confirm the positive relation between competition and bank stability among regional banks in 11 European countries. Liu, Molyneux, and Wilson (2013b) examine the competition of nine EU banking markets by using different competition measures, and conclude that different measures can yield different outcomes.
} 
where OUT is the output of a clearing and settlement institution, FIP $_{\mathrm{i}}$ are the factor input prices (regarding funding, personnel expenses, and other non-interest expenses), and $\mathrm{EX}_{\mathrm{COST}_{j}}$ are other exogenous variables to the cost function. We assume that the marginal revenue function is log-linear. That is,

$\ln \mathrm{MR}=\delta_{0}+\delta_{1} \ln \mathrm{OUT}+\sum_{\mathrm{k}=1}^{\mathrm{q}} \gamma_{\mathrm{j}} \ln \mathrm{EX}_{\mathrm{REV}}$

where $\mathrm{EX}_{\mathrm{REV}}$ are variables that define the institution-specific demand function. $\mathrm{A}$ profit-maximizing institution operates where the marginal cost equals to the marginal revenue $(\ln M C=\ln M R)$. Equating (3.1) and (3.2), we obtain

$\ln$ OUT $^{*}=\left(\alpha_{0}-\delta_{0}+\sum_{\mathrm{i}=1}^{\mathrm{m}} \beta_{\mathrm{i}} \ln \mathrm{FIP}_{\mathrm{i}}+\sum_{\mathrm{j}=1}^{\mathrm{p}} \gamma_{\mathrm{j}} \ln \mathrm{EX}_{\mathrm{COST}_{\mathrm{j}}}-\sum_{\mathrm{k}=1}^{\mathrm{q}} \gamma_{\mathrm{j}} \ln \mathrm{EX}_{\mathrm{REV}_{\mathrm{k}}}\right) /\left(\alpha_{1}-\delta_{1}\right)$

The reduced-form revenue equation is computed by multiplying equilibrium output and the common price level, which is, by the inverse-log linear-demand equation, $\ln p^{*}=\zeta+\eta \ln$ $\left(\sum_{\mathrm{i}} \mathrm{OUT}^{*}{ }_{\mathrm{i}}\right)$. We employ the following reduced-form revenue equation in our analysis:

$\ln \mathrm{OPINCOM}_{\mathrm{it}}=\alpha_{\mathrm{i}}+\beta \ln \mathrm{AFR}_{\mathrm{it}}+\gamma \ln \mathrm{PPE}_{\mathrm{it}}+\zeta \ln \mathrm{PCE}_{\mathrm{it}}+\Phi\left(\mathrm{OI}_{\mathrm{it}} / \mathrm{OR}_{\mathrm{it}}\right)+\varphi_{\mathrm{t}}+\varepsilon_{\mathrm{it}}$

where OPINCOM $_{\mathrm{it}}$ is the operating income (as a measure of the revenue) of clearing and settlement institution $i$ at year $t .{ }^{53}$ Average Funding Rate $\left(\mathrm{AFR}_{\mathrm{it}}\right)$ is the ratio of annual interest expenses to total funds. Price of Personnel Expenses $\left(\mathrm{PPE}_{\mathrm{it}}\right)$ is the ratio of personnel expenses to total assets. Price of Capital Expenditure $\left(\mathrm{PCE}_{\mathrm{it}}\right)$ is the ratio of physical capital expenditure and other expenses to fixed assets. $\mathrm{AFR}_{\mathrm{it}}, \mathrm{PPE}_{\mathrm{it}}$, and $\mathrm{PCE}_{\mathrm{it}}$ are the clearing and settlement institution's unit input prices of funding, labor, and capital. We add the ratio of other income to operating revenue $\left(\mathrm{OI}_{\mathrm{it}} / \mathrm{OR}_{\mathrm{it}}\right)$ as a control variable to account for the increasing variety of clearing and settlement activities. Following Coccorese (2009), all institution-specific and time-varying factors that could affect the level of operating income, but are not explicitly addressed in (3.4), are captured through the insertion of dummy variables associated with clearing and settlement institutions and with years (denoted by $\alpha_{\mathrm{i}}$ and $\varphi_{\mathrm{t}}$ respectively).

The PR model $(1982,1987)$ measures competition through an index ' $H$-statistic' (Bikker, et al., 2006). The $H$-statistic is defined as the sum of the elasticities of revenues with respect to input prices. In the notation of (3.4), the $H$-statistic is given by $\beta+\gamma+\zeta . H \leq 0$ indicates collusive or joint monopoly equilibrium, $0<H<1$ indicates monopolistic competition, and $H=1$ indicates perfect competition (see Panzar and Rosse, 1987). ${ }^{54}$

\footnotetext{
53 Bikker, Shaffer, and Spierdijk (2012) argue that a scaled revenue function creates a significant upward bias and incorrectly measures the degree of competition. We follow their suggestion and employ the unscaled revenue function.

${ }^{54}$ In Appendix C, Table C.3 summarizes the discriminatory power of $H$-statistic in PR model.
} 
We introduce the interaction terms between the input price variables and additional factors to analyze what drives competition in clearing and settlement.

$$
\begin{aligned}
& \ln \mathrm{OPINCOM}_{\mathrm{it}}=\alpha_{\mathrm{i}}+\beta \ln \mathrm{AFR}_{\mathrm{it}}+\gamma \ln \mathrm{PPE}_{\mathrm{it}}+\zeta \ln \mathrm{PCE}_{\mathrm{it}}+\Phi\left(\mathrm{OI}_{\mathrm{it}} / \mathrm{OR}_{\mathrm{it}}\right)+\pi_{\mathrm{j}} \mathrm{F}_{\mathrm{it}}+ \\
& \beta_{\mathrm{j}} \ln \mathrm{AFR}_{\mathrm{it}} \times \mathrm{F}_{\mathrm{itj}}+\gamma_{\mathrm{j}} \ln \mathrm{PPE}_{\mathrm{it}} \times \mathrm{F}_{\mathrm{itj}}+\zeta_{\mathrm{j}} \ln \mathrm{PCE}_{\mathrm{it}} \times \mathrm{F}_{\mathrm{itj}}+\varphi_{\mathrm{t}}+\varepsilon_{\mathrm{it}}
\end{aligned}
$$

We include several variables that may affect competition between clearing and settlement institutions $\left(\mathrm{F}_{\mathrm{itj}}\right.$ denotes one of $j$ variables). We include the global financial crisis $\left(\delta_{\mathrm{t}}\right.$, which equals to 1 during the years 2008 to 2010 and 0 otherwise), institutional structure $\left(\operatorname{ICSD}_{i}\right.$, which equals 1 for international CSDs and 0 for domestic CSDs), logarithm of institution size (Size ${ }_{i t}$ ), Merger $_{\text {it }}$ (which equals 1 on the year of a merger, and 0 otherwise), technological development

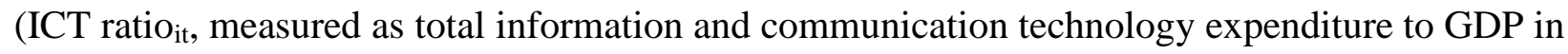
a given country), and USregion $_{\mathrm{i}}$ that equals 1 if a clearing and settlement institution is operating in the U.S. market and 0 otherwise. For the robustness check, we use the logarithm of total revenue of clearing and settlement institutions $\left(\operatorname{lnTR}_{\mathrm{it}}\right)$ as a dependent variable. All models are estimated using the ordinary least squares (OLS) regression with White's (1980) heteroskedasticity robust standard errors. Table 3.1 provides definitions of variables and data sources. All national currencies are converted into U.S. dollars and inflation-adjusted.

We define $H 0=\beta+\gamma+\zeta$ as the sum of the three input price elasticities. We compute the interaction terms of three unit input price variables and variable $j$ (i.e., $\beta_{j}+\gamma_{j}+\zeta_{j}$ ) to analyze the change of $H$-statistic due to the interaction with variable $j$. The total $H$-statistic is computed as $H$ $=\beta+\gamma+\zeta+\beta_{j}+\gamma_{j}+\zeta_{j}$ and measures the three unit input price elasticities and the regression coefficients of the interaction terms of three unit input price variables with variable $j$. 
Table 3.1. Data Structure and Source

\begin{tabular}{|c|c|c|c|}
\hline Variable & Definition & Variable Name in BankScope & Data Source \\
\hline TR & Total revenue in million dollars & (Total Operating Income + Interest Income) & BankScope \\
\hline TC & Total operating expense in million dollars & Total Operating Expense & BankScope \\
\hline OPINCOM & Total operating income in million dollars & Operating Income & BankScope \\
\hline AFR & The ratio of annual interest expenses to total funds, or other Average Funding Rate & $\begin{array}{l}\text { Total Interest Expense / (Long Term Funding + } \\
\text { Deposits \& Short Term Funding) }\end{array}$ & BankScope \\
\hline PPE & The ratio of personnel expenses to the balance sheet total asset & Personnel Expenses / Total Asset & BankScope \\
\hline PCE & The ratio of physical capital expenditure and other expenses to fixed assets & Other Operating Expenses / Fixed Assets & BankScope \\
\hline OI/OR & The ratio of other income to operating income & $\begin{array}{l}\text { (Net Income - Total Operating Income) / Total } \\
\text { Operating Income }\end{array}$ & BankScope \\
\hline$\delta$ & $\begin{array}{l}\text { A dummy variable for crises, which takes value of } 1 \text { for period } 2008-2010 \text { and } 0 \\
\text { otherwise }\end{array}$ & & \\
\hline Size & The logarithm of total assets representing the proxy for the size & Size $=\log ($ Total Asset $)$ & BankScope \\
\hline ICSD & $\begin{array}{l}\text { Binary variable, for international central securities depositories (ICSD), ICSD }=1 \text {; for } \\
\text { central securities depositories (CSD), ICSD }=0\end{array}$ & & $\begin{array}{l}\text { Annual reports } \\
1989-2012\end{array}$ \\
\hline ICT ratio & $\begin{array}{l}\text { Total information and communication technology expenditure to GDP in a given } \\
\text { country }\end{array}$ & & $\begin{array}{ll}\text { OECD } & \text { Factbook } \\
(2012) & \end{array}$ \\
\hline ROE & Return on equity & & BankScope \\
\hline Merger & $\begin{array}{l}\text { A binary variable that equals } 1 \text { on the year that the merger was announced, and } 0 \\
\text { otherwise }\end{array}$ & & \\
\hline USregion & $\begin{array}{l}\text { A dummy variable that equals to } 1 \text { if a clearing and settlement institution is from the } \\
\text { U.S., and } 0 \text { if a clearing and settlement institution is from Europe. }\end{array}$ & & \\
\hline Lerner index & $\begin{array}{l}\text { The Lerner index, an indicator of competition, derived from stochastic frontier } \\
\text { analysis (SFA) estimate of marginal cost and total assets, with higher values indicating } \\
\text { less competition. }\end{array}$ & & Own calculations \\
\hline GDP growth & Annual growth rate of GDP at market prices based on constant local currency & & World Bank \\
\hline Inflation & Inflation rate & & World Bank \\
\hline Interest rate & The interest rate charged by banks on loans to prime customers & & World Bank \\
\hline$t$ & Linear time trend variable & & \\
\hline
\end{tabular}




\subsubsection{Lerner index}

An alternative non-structural technique to the PR model is to estimate a parameter that directly measures firms' competitive behavior from the information on firm costs and demand. For example, the Lerner index is a relative mark-up of price over marginal cost and measures firm market power (Lerner, 1934). ${ }^{55}$ The higher the mark-up, the greater is the market power. The Lerner index ranges from 0 in the case of perfect competition to 1 in the case of monopoly. A number of studies (Bikker and Haaf, 2002; Shaffer, 1983a, 1983b) show empirically that the $H$-statistic and Lerner index are negatively correlated. That is, the relative price-cost mark-up (smaller Lerner index) decreases with higher competition (higher $H$-statistic). ${ }^{56}$

The Lerner index is calculated as

Lerner Index ${ }_{i t}=\left(P_{i t}-M C_{i t}\right) / P_{i t}$

where $\mathrm{P}_{\mathrm{it}}$ is the price of total assets for clearing and settlement institution $i$ at time $t$ and $\mathrm{MC}_{\mathrm{it}}$ is the marginal cost of clearing and settlement institution $i$ at time $t$. The marginal cost is derived from the total cost function. That is,

$\mathrm{MC}_{\mathrm{it}}=\frac{\mathrm{TC}_{\mathrm{it}}}{\mathrm{Q}_{\mathrm{it}}}\left(\alpha_{1}+\alpha_{2} \ln \mathrm{Q}_{\mathrm{it}}+\alpha_{9} \ln \mathrm{AFR}_{\mathrm{it}}+\alpha_{10} \ln \mathrm{PPE}_{\mathrm{it}}+\alpha_{11} \ln \mathrm{PCE}_{\mathrm{it}}\right)$

where the translog total cost function is

$\ln \mathrm{TC}_{\mathrm{it}}=$

$\alpha_{0}+\alpha_{1} \ln Q_{\mathrm{it}}+\frac{\alpha_{2}}{2}\left(\ln \mathrm{Q}_{\mathrm{it}}\right)^{2}+\alpha_{3} \ln \mathrm{AFR}_{\mathrm{it}}+\alpha_{4} \ln \mathrm{PPE}_{\mathrm{it}}+\alpha_{5} \ln \mathrm{PCE}_{\mathrm{it}}+\alpha_{6}\left(\ln \mathrm{AFR}_{\mathrm{it}}\right)^{2}+$

$\alpha_{7}\left(\ln \mathrm{PPE}_{\mathrm{it}}\right)^{2}+\alpha_{8}\left(\ln \mathrm{PCE}_{\mathrm{it}}\right)^{2}+\alpha_{9} \ln \mathrm{AFR}_{\mathrm{it}} * \ln \mathrm{Q}_{\mathrm{it}}+\alpha_{10} \ln \mathrm{PPE}_{\mathrm{it}} * \ln \mathrm{Q}_{\mathrm{it}}+\alpha_{11} \ln \mathrm{PCE}_{\mathrm{it}} *$

$\ln \mathrm{Q}_{\mathrm{it}}+\alpha_{12} \ln \mathrm{AFR}_{\mathrm{it}} * \ln \mathrm{PPE}_{\mathrm{it}}+\alpha_{13} \ln \mathrm{PPE}_{\mathrm{it}} * \ln \mathrm{PCE}_{\mathrm{it}}+\alpha_{14} \ln \mathrm{PCE}_{\mathrm{it}} * \ln \mathrm{AFR}_{\mathrm{it}}+\varepsilon_{\mathrm{it}}$

and $\mathrm{TC}_{\mathrm{it}}$ represents total costs measured by the total operating expenses and $\mathrm{Q}_{\text {it }}$ represents the output, measured by the total assets of a clearing and settlement institution $i$. $\mathrm{AFR}_{\mathrm{it}}, \mathrm{PPE}_{\mathrm{it}}$, and $\mathrm{PCE}_{\mathrm{it}}$ represent the input prices of the clearing and settlement institution, as defined previously in the PR model. Following Fu et al. (2014), Koetter et al. (2012), and Kumbhakar and Lovell (2000), we use the stochastic cost frontier analysis to estimate (3.8).

We then estimate the Lerner index in (3.6) by using the marginal cost based on (3.7) and the

\footnotetext{
55 The Lerner index is widely employed to estimate competition in the banking sector. Coccorese (2009) points out that Lerner index reflects well the bank's level of market power. Angelini and Cetorelli (2003) assess the behavior of Italian regional banks and find that deregulation led to a reduction in price-costs margins. See also Koetter et al. (2012), and Fu et al. (2014).

56 Several other approaches have been developed that mostly build on the Lerner measure of market power. For example, Bresnahan (1982) and Lau (1982) estimate the conjectural variation coefficient based on the deviation of perceived firm revenues from demand. A high conjectural variation suggests that a firm is highly aware of anticipates strongly its interdependence with other firms when setting the level of output and prices (see also Appelbaum, 1979; Iwata, 1974).
} 
price of total assets $\mathrm{P}_{\mathrm{it}}$ proxied by the ratio of total revenues to total assets. Subsequently, we estimate the following regression:

Lerner index ${ }_{\mathrm{it}}=\beta_{0}+\sum_{j} \beta_{\mathrm{k}}$ Controls $_{\mathrm{itj}}+\sum_{j} \beta_{\mathrm{j}} \mathrm{F}_{\mathrm{itj}}+\varepsilon_{\mathrm{it}}$

We are interested in how several factors $F_{i t j}$ affect competition. We analyze the effect of the global financial crisis $\left(\delta_{t}\right)$, institutional structure $\left(\operatorname{ICSD}_{i}\right)$, institutional size $\left(\operatorname{Size}_{\mathrm{it}}\right)$, the effects of mergers of different institutions (Merger ${ }_{i t}$ ), and the geographic location (USregion ${ }_{\mathrm{i}}$ ) on Lerner inde $_{\mathrm{it}}$. In an additional specification, we also include ICT ratio $\mathrm{it}_{\mathrm{it}}$ and a time trend variable $(t)$ to capture the effect of technological development.

As control variables, Controls itj $_{\text {, }}$, we include GDP growth and inflation in a country to account for economic cycles. We also add interest rates and the number of clearing and settlement institutions to control for the changes of monetary policy and the market structure of the clearing and settlement industry in a given country. We estimate the regression in (3.9) by using the feasible generalized least squares (FGLS) approach to cope with the heteroskedasticity problem.

\subsubsection{Boone indicator}

A more recent measure of competition is proposed by Boone $(2001,2008)$. The Boone indicator captures the link between competition and efficiency. It builds on the efficient structure hypothesis that relates firm performance with differences in efficiency. In particular, firms that are more efficient also perform superiorly which results in higher profits. The idea behind the Boone indicator is that the relationship between efficiency and profits is increasing in the degree of competition. The Boone model can be characterized as

$\ln \pi_{i t}=\alpha+\beta \ln \mathrm{MC}_{i t}+\varepsilon_{i t}$

where $\pi_{i t}$ is the profit, $\mathrm{MC}_{i t}$ is the marginal cost of a CSD $i$ at year $t$, and $\beta$ is defined as the Boone indicator. The Boone indicator $\beta$ is negative and decreasing in the level of competition. We use the log-log specification to better deal with heteroskedasticity.

$\ln \pi_{\mathrm{it}}=\alpha+\beta \ln \mathrm{MC}_{i t}+\sum_{j} \beta_{\mathrm{j}} \mathrm{F}_{\mathrm{itj}} * \ln \mathrm{MC}_{i t}+\varepsilon_{\mathrm{it}}$

We are interested in how several factors $F_{i t j}$, including the global financial crisis $\left(\delta_{t}\right)$, institutional structure $\left(\operatorname{ICSD}_{i}\right)$, institutional size $\left(\right.$ Size $\left._{i t}\right)$, the effects of mergers of different institutions $\left(\right.$ Merger $_{i t}$ ), ICT ratio ${ }_{i t}$, and the geographic location $\left(\mathrm{USregion}_{\mathrm{i}}\right.$ ), affect the Boone indicator. We include the interaction terms of a marginal cost and factors $F_{i t j}$ to analyze the change of Boone indicator due to the interaction with variable $\mathrm{j}$ (captured in $\beta_{\mathrm{j}}$ ).

By computing the marginal cost from (3.7), we estimate the Boone indicator from (3.10) and (3.11). We use the feasible generalized least squares approach to cope with the heteroskedasticity 
problem. ${ }^{57}$

We estimate concentration indexes, $H$-statistic, Lerner index, and the Boone indicator to measure competition in clearing and settlement.

\subsection{Description of Data and Concentration Measures}

The data is obtained from several sources, including BankScope database, OECD factbook, World Bank, and annual reports of the clearing and settlement institutions between 1989 and 2012. We focus on the U.S. and the European domestic and international clearing and settlement institutions (see Table 3.2).

Table 3.3 provides descriptive statistics of revenues and costs of the clearing and settlement institutions, based on geographic location and institutional type. Substantial variability across variables indicates that the diversity of economic conditions, changes in technological development, and the variety of services provided affect the characteristics of clearing and settlement institutions.

We can compare average cost structure of clearing and settlement institutions across the U.S. and EU. In the sample period, European clearing and settlement institutions have significantly higher interest expenses and physical expenses, but lower personnel expenses compared to U.S. clearing and settlement institutions.

We can also compare characteristics of ICSDs and CSDs. Personnel expenses and physical expenses are higher for ICSDs than for CSDs. The total revenue of ICSDs is $\$ 881.2$ million; this is 2.5 times as high as the total revenue of CSDs (\$358.56 million). That the average personnel expenses and physical expenses are higher for ICSDs compared to CSDs is consistent with Lannoo and Levin's (2002) findings that the operating costs of an ICSD are substantially higher than the operating costs of a CSD.

Table 3.2 also contains Herfindahl-Hirschman index and CR3 concentration index across countries in our sample. Both indicators suggest that clearing and settlement services are highly concentrated. As captured by our dataset, a single CSD operates in several countries. European security markets are substantially fragmented along national lines. The question is whether cross-border competition between CSDs can still exist in such a fragmented environment.

\footnotetext{
57 The FGLS estimator has similar properties as the GLS estimator, such as consistency and asymptotic normality (White, 1980).
} 
Table 3.2. Summary of Sample Clearing and Settlement Institutions, 1989-2012

\begin{tabular}{|c|c|c|c|c|c|}
\hline Clearing and Settlement Institution & CSD/ICSD & Years & Country & HHI & CR3 \\
\hline Oesterreichische Kontrollbank AG & CSD & $2002-2012$ & Austria & 1 & 1 \\
\hline Euroclear Bank & ICSD & $2000-2010$ & Belguim & \multirow{2}{*}{0.5296} & \multirow{2}{*}{1} \\
\hline Euroclear SA/NV & $\mathrm{CSD}$ & 2005-2010 & Belguim & & \\
\hline Central Registry of Securities JSC-Republic of Srpska & CSD & 2005-2012 & Bosnia \& Herzegovina & 1 & 1 \\
\hline Central Depository AD & CSD & $2007-2012$ & Bulgaria & 1 & 1 \\
\hline Central Depository \& Clearing Company Inc & CSD & $2008-2012$ & Croatia & 1 & 1 \\
\hline Cyprus Stock Exchange & $\mathrm{CSD}$ & 2003-2012 & Cyprus & 1 & 1 \\
\hline Central Securities Depository Prague & CSD & $1999-2011$ & Czech Republic & 1 & 1 \\
\hline VP Securities Service & CSD & 2006-2012 & Denmark & 1 & 1 \\
\hline Banque Centrale de Compensation & CSD & $2001-2010$ & France & \multirow{5}{*}{0.3558} & \multirow{5}{*}{1} \\
\hline CACEIS Bank France & CSD & 2005-2010 & France & & \\
\hline Euroclear France & CSD & $1999-2000$ & France & & \\
\hline Euronext Paris SA & CSD & $1996-2000,2009-2010$ & France & & \\
\hline IXIS Investor Services & CSD & 2005 & France & & \\
\hline Clearstream Banking AG Frankfurt & CSD & $1995-2010$ & Germany & \multirow{3}{*}{0.7717} & \multirow{3}{*}{1} \\
\hline European Commodity Clearing AG & CSD & $2008-2010$ & Germany & & \\
\hline Swiss Euro Clearing Bank GmbH & $\mathrm{CSD}$ & $2000-2010$ & Germany & & \\
\hline KELER Ltd & CSD & 2001-2012 & Hungary & 1 & 1 \\
\hline Iceland Securities Depository & CSD & $2010-2012$ & Iceland & 1 & 1 \\
\hline Cedel International & ICSD & 1993-1999 & Luxembourg & \multirow{6}{*}{0.5011} & \multirow{6}{*}{1} \\
\hline Centre de Transferts Electronique & CSD & 2002-2005 & Luxembourg & & \\
\hline Clearstream Banking SA & CSD & 1995-2010 & Luxembourg & & \\
\hline Clearstream International & ICSD & $2000-2006$ & Luxembourg & & \\
\hline Clearstream Services SA & CSD & $1999-2000$ & Luxembourg & & \\
\hline RBC Dexia Investor Services Bank & CSD & 2003-2010 & Luxembourg & & \\
\hline Malta Stock Exchange & CSD & $2000-2012$ & Malta & 1 & 1 \\
\hline ABN AMRO Clearing Bank N.V. & CSD & $2004-2010$ & Netherlands & \multirow{4}{*}{0.5226} & \multirow{4}{*}{1} \\
\hline CITCO Bank Nederland NV & CSD & $1994-2010$ & Netherlands & & \\
\hline Fortis Clearing International B.V & CSD & 1999 & Netherlands & & \\
\hline RBC Dexia Investor Services Nethe & CSD & $2005-2006$ & Netherlands & & \\
\hline
\end{tabular}


Table 3.2. Summary of Sample Clearing and Settlement Institutions, 1989-2012

\begin{tabular}{|c|c|c|c|c|c|}
\hline Clearing and Settlement Institution & CSD/ICSD & Years & Country & HHI & CR3 \\
\hline KDPW & CSD & $2002-2011$ & Poland & 1 & 1 \\
\hline Moscow Clearing Centre-Moskovsky & CSD & $2009-2011$ & Russia & \multirow{3}{*}{0.3649} & \multirow{3}{*}{1} \\
\hline National Clearing Centre CJSC JSC & CSD & $2007-2010$ & Russia & & \\
\hline National Settlement Depository & CSD & 2009-2012 & Russia & & \\
\hline Central Securities Depository of the Slovak Republic & CSD & $2008-2012$ & Slovak Republic & 1 & 1 \\
\hline Central Securities Clearing Corporation & CSD & $2007-2012$ & Slovenia & 1 & 1 \\
\hline RBC Dexia Investor Services Espan & CSD & $1989-2010$ & Spain & 1 & 1 \\
\hline CLS Group Holdings AG & CSD & $2004-2010$ & Switzerland & \multirow{2}{*}{0.8207} & \multirow{2}{*}{1} \\
\hline SIX Swiss Exchange & CSD & $2007-2012$ & Switzerland & & \\
\hline Central Securities Depository of Turkey & CSD & $2004-2012$ & Turkey & \multirow{2}{*}{0.5006} & \multirow{2}{*}{1} \\
\hline Takasbank-Istanbu Settlement and Custody Bank Inc & CSD & 1999-2012 & Turkey & & \\
\hline Euroclear Plc & CSD & $1999-2010$ & United Kingdom & \multirow{4}{*}{0.4706} & \multirow{4}{*}{1} \\
\hline LCH Clearnet Group Limited & CSD & $2002-2011$ & United Kingdom & & \\
\hline LCH.Clearnet Limited & CSD & 2006-2011 & United Kingdom & & \\
\hline RBSI Custody Bank Limited & CSD & 2001-2002, 2004-2005 & United Kingdom & & \\
\hline Fixed Income Clearing Corporation & CSD & 2003-2012 & U.S. & \multirow{4}{*}{0.5822} & \multirow{4}{*}{1} \\
\hline National Securities Clearing Corporation & CSD & $2003-2012$ & U.S. & & \\
\hline The Depository Trust Company & ICSD & $2003-2012$ & U.S. & & \\
\hline The Depository Trust \& Clearing Corporation & ICSD & 2003-2012 & U.S. & & \\
\hline
\end{tabular}

Note. 1) HHI is the Herfindahl Index, defined as HHI $=\sum_{i} m_{i}^{2}$ is the sum of the squared market shares of each clearing and settlement institutions at 2010 in each country. 2) CR3 is the share of the market taken by the largest three clearing and settlement institutions at 2010 in each country. 3) The estimation of HHI and CR3 is based on the data in 2010. 4) The data for BNY Mellon CSD and NBB SSS in Belgium was not available. 5) The data for Euroclear Netherlands in Netherlands was not available. 6) The data for Central Register of Treasury Bills (CRBS) in Poland was not available. 7) The data for Iberclear in Spain was not available. 
Table 3.3. Data Statistics

Variable

Operating Income

(\$ million)

Total Revenue

(\$ million)

Interest Expenses

(\$ million)

Personnel

Expenses

Physical Expenses

(\$ million)

Fixed Asset

(\$ million)

Total Asset

(\$ million)

AFR

PPE

PCE

OI/OR

ROE $(\%)$

ICT $(\%)$
Total

284.20

$(-2.88-8,876)$

414.87

$(-2.28-8,903)$

113.05

(0.01-1,816)

79.32

(0.06-579)

129.06

(0.79-8,841)

35.78

(0.00-256)

33,178

(13.21-700,049)

0.13

(0.00-7.00)

0.15

(0.00-0.51)

5.67

(0.20-71.34)

0.69

(-0.58-8.62)

11.5

(-64.45-144.01)

12.17

9.56-32.10)

\begin{tabular}{|c|c|c|c|}
\multicolumn{2}{c}{ Regions } & \multicolumn{2}{c|}{ ICSD / CSD } \\
\hline Europe & U.S. & ICSD & CSD \\
258.78 & 686.19 & 680.41 & 236.10 \\
$(-2.88-8,876)$ & $(341-1,089)$ & $(131.44-1,772)$ & $(-2.88-8,876)$ \\
391.27 & 787.16 & 881.18 & 358.56 \\
$(-2.28-8,903)$ & $(361-1,555)$ & $(300-2,711)$ & $(-2.28-8,903)$ \\
118.92 & 21.51 & 67.32 & 118.83 \\
$(0.01-1,816)$ & $(14-26.1)$ & $(0.12-481)$ & $(0.01-1,816)$ \\
63.06 & 207.49 & 269.84 & 55.98 \\
$(0.36-579)$ & $(0.06-532)$ & $(26.73-559)$ & $(0.06-579)$ \\
137.62 & 63.63 & 203.65 & 168.53 \\
$(0.79-8,841)$ & $(8.41-213)$ & $(29.89-915)$ & $(0.79-8,841)$ \\
28.62 & 95.08 & 126.79 & 18.34 \\
$(0.00-200)$ & $(8.11-256)$ & $(16.27-256)$ & $(0.00-201)$ \\
36,586 & 13,303 & 11,623 & 36,873 \\
$(13.21-700,049)$ & $(2,241-50,898)$ & $(1,012-50,898)$ & $(13.21-700,049)$ \\
0.14 & 0.01 & 0.05 & 0.15 \\
$(0.00-7.00)$ & $(0.00-0.05)$ & $(0.00-0.75)$ & $(0.01-7.00)$ \\
0.17 & 0.03 & 0.03 & 0.16 \\
$(0.00-0.51)$ & $(0.00-0.10)$ & $(0.01-0.10)$ & $(0.00-0.51)$ \\
6.64 & 1.16 & 2.16 & 6.64 \\
$(0.39-71.34)$ & $(0.20-10.86)$ & $(0.20-10.86)$ & $(0.39-71.34)$ \\
0.72 & 0.17 & 0.38 & 0.73 \\
$(-0.58-8.62)$ & $(0.04-0.49)$ & $(0.04-1.29)$ & $(-0.58-8.62)$ \\
11.29 & 12.5 & 11.64 & 11.4 \\
$(-64.45-144.01)$ & $(-13.79-57.19)$ & $(-9.88-57.19)$ & $(-64.45-144.01)$ \\
9.09 & 29.28 & 29.27 & 10.72 \\
$(9.56-25.00)$ & $(26.30-32.10)$ & $(26.30-32.10)$ & $(9.56-32.10)$ \\
\hline
\end{tabular}

Note. This table describes the mean of each variable, and range of each variable is reported in parentheses. All currencies are converted to dollars and inflation adjusted.

Despite high concentration, competition between several providers might still be possible in a contestable market when the threat of new entry forces a local monopolist to charge competitive prices. The improved services of ICSDs and their links to local CSDs might have contributed to increased competition in the clearing and settlement industry. Competitive pressure by ICSDs, which are increasingly acquiring the local CSDs, is threatening the position of local CSDs in the financial markets. Through the threat of the entry, ICSDs might then establish a more competitive conduct of local CSDs (Van Cayseele, 2004). In this case, the structural approach to measuring competition through HHI and CR3 is inadequate. Therefore, further test of conduct should aim at directly addressing the competitive behavior of clearing and settlement institutions.

\subsection{Empirical Analysis Based on Panzar-Rosse Model}

We now estimate $H$-statistic based on the unscaled PR model as presented in (3.4) and (3.5). The results of the empirical estimation are reported in Table 3.4. In Panel A, we employ the operating income as a dependent variable. Column 1 of Panel A shows that the unit price of labor is negatively and statistically significantly related to the operating income, whereas the unit prices of funding and capital are positively but insignificantly associated with the operating income. The elasticity of the unit price of labor, $\gamma$, is the largest, followed by the unit price of capital, $\varsigma$, and then by the unit price of funding, $\beta$. This shows that the personnel expenses are the main input factor in clearing and settlement services. When we add the global financial crisis dummy 
$\left(\delta_{t}\right)$ in the regression, the regression coefficients of unit input price variables do not change substantially.

Table 3.4. The Competitive Equilibrium and the Impact of the Global Financial Crisis on H-statistic of Clearing and Settlement Institutions

\begin{tabular}{|c|c|c|c|c|c|c|c|}
\hline \multirow[t]{2}{*}{ Variable } & \multirow[t]{2}{*}{ Coefficients } & \multicolumn{3}{|c|}{ Panel A: InOPINCOM } & \multicolumn{3}{|c|}{ Panel B: $\ln T R$} \\
\hline & & (1) & (2) & (3) & (1) & (2) & (3) \\
\hline \multirow[t]{2}{*}{$\ln \mathrm{AFR}$} & $\beta$ & 0.0318 & 0.0301 & 0.0214 & 0.0521 & 0.0505 & 0.0411 \\
\hline & & $(0.36)$ & $(0.34)$ & $(0.23)$ & $(0.63)$ & $(0.61)$ & $(0.48)$ \\
\hline \multirow[t]{2}{*}{$\operatorname{lnPPE}$} & $\gamma$ & $-0.149 *$ & $-0.151 *$ & $-0.168 *$ & $-0.177 * *$ & $-0.178 * *$ & $-0.198 * *$ \\
\hline & & $(-1.65)$ & $(-1.66)$ & $(-1.75)$ & $(-2.02)$ & $(-2.03)$ & $(-2.12)$ \\
\hline \multirow[t]{2}{*}{$\operatorname{lnPCE}$} & $\zeta$ & 0.0403 & 0.0399 & -0.0159 & 0.0398 & 0.0395 & -0.0127 \\
\hline & & $(0.58)$ & $(0.57)$ & $(-0.32)$ & $(0.60)$ & $(0.60)$ & $(-0.27)$ \\
\hline \multirow[t]{2}{*}{$\mathrm{OI} / \mathrm{OR}$} & $\psi$ & -0.152 & -0.154 & -0.137 & $0.162 *$ & $0.160 *$ & $0.175^{*}$ \\
\hline & & $(-1.53)$ & $(-1.55)$ & $(-1.49)$ & $(1.68)$ & $(1.66)$ & $(1.94)$ \\
\hline \multirow[t]{2}{*}{$\delta$} & $\Pi_{1}$ & & -0.313 & 0.0404 & & -0.298 & 0.105 \\
\hline & & & $(-1.26)$ & $(0.15)$ & & $(-1.19)$ & $(0.36)$ \\
\hline \multirow[t]{2}{*}{$\ln \mathrm{AFR} * \delta$} & $\beta_{1}$ & & & 0.0177 & & & 0.0173 \\
\hline & & & & $(0.79)$ & & & $(0.75)$ \\
\hline \multirow[t]{2}{*}{$\operatorname{lnPPE} * \delta$} & $\gamma_{1}$ & & & 0.0571 & & & 0.0682 \\
\hline & & & & $(1.38)$ & & & $(1.62)$ \\
\hline \multirow[t]{2}{*}{$\ln \mathrm{PCE} * \delta$} & $\zeta_{1}$ & & & $0.133 * *$ & & & $0.124 * *$ \\
\hline & & & & $(2.35)$ & & & $(2.31)$ \\
\hline \multicolumn{2}{|c|}{$H 0$-statistic $(\beta+\gamma+\zeta)$} & -0.0769 & -0.0810 & -0.1625 & -0.0851 & -0.0880 & -0.1696 \\
\hline \multicolumn{2}{|c|}{ Wald $H O \leq 0$ ( $p$-value $)$} & 0.6870 & 0.6923 & 0.8611 & 0.7073 & 0.7120 & 0.8684 \\
\hline \multicolumn{2}{|c|}{ Wald $H O=1(p$-value $)$} & 0.0000 & 0.0000 & 0.0000 & 0.0000 & 0.0000 & 0.0000 \\
\hline \multicolumn{2}{|l|}{$\beta_{1}+\gamma_{1}+\zeta_{1}$} & & & 0.2078 & & & 0.2095 \\
\hline \multicolumn{2}{|c|}{ Wald $\beta_{1}+\gamma_{1}+\zeta_{1} \leq 0$ ( $p$-value) } & & & 0.0130 & & & 0.0102 \\
\hline \multicolumn{2}{|l|}{$H$-statistic } & & & 0.0453 & & & 0.0399 \\
\hline \multicolumn{2}{|c|}{ Wald $H \leq 0$ ( $p$-value $)$} & & & 0.3960 & & & 0.4058 \\
\hline \multicolumn{2}{|c|}{ Wald $H=1$ ( $p$-value $)$} & & & 0.0000 & & & 0.0000 \\
\hline \multicolumn{2}{|c|}{ Equilibrium test (ROE) ( $p$-value) } & 0.4073 & 0.4504 & 0.5262 & 0.4073 & 0.4054 & 0.5262 \\
\hline \multicolumn{2}{|l|}{$N$} & 318 & 318 & 318 & 318 & 318 & 318 \\
\hline \multicolumn{2}{|c|}{ Adjusted-R2 } & 0.986 & 0.986 & 0.987 & 0.994 & 0.994 & 0.994 \\
\hline
\end{tabular}

Note. The dependent variable lnOPINCOM represents log of operating income; lnTR represents log of total revenue. All regressions are OLS estimation. Dummy variables associated with clearing and settlement institutions and years are included. Heteroskedasticity robust $t$-values are reported in parentheses. Superscripts $* * *, * *, *$ indicate significant level of $0.01,0.05$ and 0.10 , respectively. The $p$-values of the Wald tests are also provided.

The $H$-statistic $(\beta+\gamma+\zeta)$ in columns 1 and 2 of Table 3.4 equals to -0.0769 and -0.810 respectively, and the Wald test shows that $H \leq 0$ cannot be rejected. This confirms that clearing and settlement institutions operate under monopoly (or under collusion).

An important feature of the $H$-statistic is that the PR model must be based on firms that operate in a long-run equilibrium (Nathan and Neave, 1989; Panzar and Rosse, 1987). Shaffer (1982) suggests an equilibrium ROE test that uses the return on equity instead of total operating income as the independent variable in (3.4) to check whether firms operate in a long-run equilibrium (Bikker, et al., 2012; see also Claessens and Laeven, 2004). Null hypothesis $H_{0}: H^{R O E}=0$ suggests the long-run equilibrium and $H_{1}: H^{R O E}<0$ confirms disequilibrium. Based on a one-sided $t$-test, we can find that the hypothesis of the long-run equilibrium $\left(H^{R O E}=0\right)$ cannot be rejected (see $p$-value of ROE test in the equilibrium test in Table 3.4). Hence, our findings indicate that clearing and settlement institutions operate under monopoly in a long-run 
equilibrium.

We now examine factors that may affect competition in clearing and settlement.

The global financial crisis: The global financial crisis has no significant effect on the operating income of clearing and settlement institutions. The Wald test indicates that the $H$-statistic significantly increased during the global financial crisis $\left(\beta_{1}+\gamma_{1}+\zeta_{1}=0.2078\right.$ and the hypothesis that $\beta_{1}+\gamma_{1}+\zeta_{1} \leq 0$ is rejected at $5 \%$ level). This indicates that the competition between clearing and settlement institutions is higher during the global financial crisis than during the normal times, and this is consistent with our Hypothesis 3.1. As a robustness check, we also use total revenues as a dependent variable to obtain the same results (see Panel B of Table 3.4).

Institutional structure: Binary variable $\operatorname{ICSD}_{\mathrm{i}}$ is positively and statistically significantly related to the operating income of a clearing and settlement institution (see Table 3.5). An institution that operates cross-border securities clearing and settlements is able to secure larger operating income potentially due to a wider range of services, instruments, and products that bring more business and higher revenues (this finding is consistent with the comparison between ICSDs and CSDs in Table 3.3).

To estimate the direct effect of dummy variable $\operatorname{ICSD}_{\mathrm{i}}$ on $H$-statistic, we test the statistical significance of the sign of the interaction term $\left(\beta_{2}+\gamma_{2}+\zeta_{2}\right)$. The interaction term equals 0.4280 (Panel A) and 0.3994 (Panel B) respectively, and the hypothesis that $\beta_{2}+\gamma_{2}+\zeta_{2} \leq 0$ is rejected. This result is consistent with Hypothesis 3.2 that claims that competition between ICSDs is higher than competition between domestic CSDs.

One explanation is that ICSDs that provide cross-border services do not only compete with the CSDs in the local market, but also with the CSDs from other countries and with other ICSDs. This finding indicates that several barriers to cross-border clearing and settlement (as identified by the Giovannini group $(2002,2003)$ ) might not substantially lower competition among ICSDs (despite making cross-border clearing and settlement substantially more expensive; see Van Cayseele and Wuyts (2007)).

Table 3.5. The Impact of Institutional Structure (ICSD) on H-statistic of Clearing and Settlement Institutions

\begin{tabular}{|c|c|c|c|c|c|}
\hline \multirow[t]{2}{*}{ Variable } & \multirow[t]{2}{*}{ Coefficients } & \multicolumn{2}{|c|}{ Panel A: InOPINCOM } & \multicolumn{2}{|c|}{ Panel B: $\ln T R$} \\
\hline & & & (2) & (1) & (2) \\
\hline \multirow[t]{2}{*}{$\ln \mathrm{AFR}$} & $\beta$ & 0.0318 & 0.0321 & 0.0521 & 0.0522 \\
\hline & & $(0.36)$ & $(0.34)$ & $(0.63)$ & $(0.60)$ \\
\hline \multirow[t]{2}{*}{$\operatorname{lnPPE}$} & $\gamma$ & $-0.149 *$ & $-0.157 *$ & $-0.177 * *$ & $-0.184 * *$ \\
\hline & & $(-1.65)$ & $(-1.70)$ & $(-2.02)$ & $(-2.06)$ \\
\hline \multirow[t]{2}{*}{$\operatorname{lnPCE}$} & $\zeta$ & 0.0403 & 0.0367 & 0.0398 & 0.0370 \\
\hline & & $(0.58)$ & $(0.51)$ & $(0.60)$ & $(0.55)$ \\
\hline \multirow[t]{2}{*}{$\mathrm{OI} / \mathrm{OR}$} & $\psi$ & -0.152 & -0.153 & $0.162 *$ & $0.161 *$ \\
\hline & & $(-1.53)$ & $(-1.52)$ & $(1.68)$ & $(1.66)$ \\
\hline \multirow[t]{2}{*}{ ICSD } & $\Pi_{2}$ & $1.362 *$ & $2.425 * *$ & $1.440 *$ & $2.471 * *$ \\
\hline & & $(1.75)$ & $(2.23)$ & $(1.78)$ & $(2.18)$ \\
\hline $\ln \mathrm{AFR} * \mathrm{ICSD}$ & $\beta_{2}$ & & -0.157 & & -0.150 \\
\hline
\end{tabular}


Table 3.5. The Impact of Institutional Structure (ICSD) on H-statistic of Clearing and Settlement Institutions

\begin{tabular}{|c|c|c|c|c|c|}
\hline \multirow[t]{3}{*}{ Variable } & \multirow[t]{3}{*}{ Coefficients } & \multicolumn{2}{|c|}{ Panel A: InOPINCOM } & \multicolumn{2}{|c|}{ Panel B: $\ln T R$} \\
\hline & & (1) & (2) & & (2) \\
\hline & & & $(-1.14)$ & & $(-1.06)$ \\
\hline \multirow[t]{2}{*}{$\operatorname{lnPPE} *$ ICSD } & $\gamma_{2}$ & & $0.617 * * *$ & & $0.605^{* * *}$ \\
\hline & & & $(3.46)$ & & $(3.24)$ \\
\hline \multirow[t]{2}{*}{$\operatorname{lnPCE} *$ ICSD } & $\zeta_{2}$ & & -0.0320 & & -0.0556 \\
\hline & & & $(-0.28)$ & & $(-0.46)$ \\
\hline$H O$-statistic $(\beta+\gamma+\zeta)$ & & -0.0769 & -0.0882 & -0.0851 & -0.0948 \\
\hline Wald $H O \leq 0$ ( $p$-value $)$ & & 0.6870 & 0.7013 & 0.7073 & 0.7191 \\
\hline Wald $H O=1$ ( $p$-value $)$ & & 0.0000 & 0.0000 & 0.0000 & 0.0000 \\
\hline$\beta_{2}+\gamma_{2}+\zeta_{2}$ & & & 0.4280 & & 0.3994 \\
\hline Wald $\beta_{2}+\gamma_{2}+\zeta_{2} \leq 0$ ( $p$-value) & & & 0.0382 & & 0.0528 \\
\hline$H$-statistic $\left(\beta+\gamma+\zeta+\beta_{2}+\gamma_{2}+\zeta_{2}\right)$ & & & 0.3398 & & 0.3046 \\
\hline Wald $H \leq 0$ ( $p$-value $)$ & & & 0.0161 & & 0.0383 \\
\hline Wald $H=1$ ( $p$-value $)$ & & & 0.0000 & & 0.0000 \\
\hline Equilibrium test (ROE) ( $p$-value) & & 0.4073 & 0.2245 & 0.4073 & 0.2245 \\
\hline$N$ & & 318 & 318 & 318 & 318 \\
\hline Adjusted-R2 & & 0.986 & 0.986 & 0.994 & 0.994 \\
\hline
\end{tabular}

Note. The dependent variable lnOPINCOM represents log of operating income; lnTR represents log of total revenue. All regressions are OLS estimation. Dummy variables associated with clearing and settlement institutions and years are included. Heteroskedasticity robust $t$-values are reported in parentheses. Superscripts $* * *, * *, *$ indicate significant level of $0.01,0.05$ and 0.10 , respectively. The $p$-values of the Wald tests are also provided.

Institutional size and merger: We estimate the interaction between $H$-statistic and i) institution size $\left(\right.$ Size $_{\mathrm{it}}$ ), measured by the logarithm of total asset, and ii) binary variable Merger ${ }_{i t}$. Variable Size $_{i t}$ is positively and statistically significantly related to total revenues. This indicates that large clearing and settlement institutions have higher revenues than small ones. The regression coefficient of the interaction terms between $H$-statistics and institution size, $\beta_{3}+\gamma_{3}+\zeta_{3}$, is statistically significantly positive (see Table 3.6). ${ }^{58}$ This is consistent with the positive relationship between the competition and the size of clearing and settlement institutions as predicted in Hypothesis 3.3..$^{59}$

The regression coefficient of the interaction term between the $H$-statistics and dummy variable Merger $_{i t}, \beta_{4}+\gamma_{4}+\zeta_{4}$, equals 0.2393 and is statistically significantly positive (see Table 3.7 ). This is consistent with the positive relationship between competition and increased merger activity as predicted by Hypothesis 3.4. According to Tapking and Yang (2006), mergers lower operating costs (the link between merged CSDs can be terminated after a full technical merger (Tapking and Yang, 2006)). Our findings indicate that clearing and settlement institutions might exploit such lower operating costs to compete for their customers more intensively.

\footnotetext{
58 The inclusion of scale in estimation of $H$-statistic results in a significant upward bias and an incorrect measure of the degree of competition (Bikker, et al., 2012). The estimated $H$-statistic $(\beta+\gamma+\zeta)$ in Table 3.6 is based on a scaled revenue function and is therefore not considered for evaluation of competitive conditions.

59 These findings resemble observations in the banking industry. Bikker and Groeneveld (2000), De Bandt and Davis (2000), Bikker and Haaf (2002), Hempell (2002), and Bikker (2004) find that competition in banking increases with a bank's size.
} 
Table 3.6. The Impact of Size on H-statistic of Clearing and Settlement Institutions

\begin{tabular}{|c|c|c|c|c|c|}
\hline \multirow{3}{*}{$\begin{array}{l}\text { Variable } \\
\ln \mathrm{AFR}\end{array}$} & \multirow{3}{*}{$\begin{array}{l}\text { Coefficients } \\
\beta\end{array}$} & \multicolumn{2}{|c|}{ Panel A: InOPINCOM } & \multicolumn{2}{|c|}{ Panel B: lnTR } \\
\hline & & & (2) & (1) & $(2)$ \\
\hline & & 0.0398 & $0.332 *$ & 0.0607 & $0.288 *$ \\
\hline & & $(0.46)$ & $(1.80)$ & $(0.77)$ & $(1.66)$ \\
\hline \multirow[t]{2}{*}{$\operatorname{lnPPE}$} & $\gamma$ & $0.149 *$ & -0.137 & $0.145^{*}$ & -0.140 \\
\hline & & $(1.83)$ & $(-1.05)$ & $(1.94)$ & $(-1.15)$ \\
\hline \multirow[t]{2}{*}{$\ln P C E$} & $\zeta$ & 0.0599 & $-0.131 *$ & 0.0610 & $-0.130 *$ \\
\hline & & $(0.88)$ & $(-1.90)$ & $(0.95)$ & $(-1.88)$ \\
\hline \multirow[t]{2}{*}{$\mathrm{OI} / \mathrm{OR}$} & $\psi$ & $-0.214 * *$ & $-0.241 * * *$ & 0.0950 & 0.0684 \\
\hline & & $(-2.15)$ & $(-2.60)$ & $(1.05)$ & $(0.84)$ \\
\hline \multirow[t]{2}{*}{ Size } & $\Pi_{3}$ & $0.434 * * *$ & $0.450 * * *$ & $0.468 * * *$ & $0.499 * * *$ \\
\hline & & $(5.43)$ & $(5.39)$ & $(6.37)$ & $(6.38)$ \\
\hline \multirow[t]{2}{*}{$\ln \mathrm{AFR} * \mathrm{Size}$} & $\beta_{3}$ & & $-0.0295^{*}$ & & -0.0230 \\
\hline & & & $(-1.80)$ & & $(-1.49)$ \\
\hline \multirow[t]{2}{*}{$\operatorname{lnPPE} *$ Size } & $\gamma_{3}$ & & $0.0325 * *$ & & $0.0334 * * *$ \\
\hline & & & $(2.47)$ & & $(2.72)$ \\
\hline \multirow[t]{2}{*}{$\operatorname{lnPCE} *$ Size } & $\zeta_{3}$ & & $0.0265 * *$ & & $0.0265^{* *}$ \\
\hline & & & $(2.16)$ & & $(2.26)$ \\
\hline \multicolumn{2}{|l|}{$H O$-statistic $(\beta+\gamma+\zeta)$} & 0.0997 & 0.0640 & 0.2667 & 0.018 \\
\hline \multicolumn{2}{|l|}{ Wald $H O \leq 0$ ( $p$-value $)$} & 0.0592 & 0.3959 & 0.0360 & 0.4680 \\
\hline \multicolumn{2}{|l|}{ Wald $H O=1$ ( $p$-value $)$} & 0.0000 & 0.0000 & 0.0000 & 0.0000 \\
\hline \multicolumn{2}{|l|}{$\beta_{3}+\gamma_{3}+\zeta_{3}$} & & 0.0295 & & 0.0369 \\
\hline \multicolumn{2}{|l|}{ Wald $\beta_{3}+\gamma_{3}+\zeta_{3} \leq 0$ ( $p$-value $)$} & & 0.0813 & & 0.0347 \\
\hline \multicolumn{2}{|l|}{$H$-statistic $\left(\beta+\gamma+\zeta+\beta_{3}+\gamma_{3}+\zeta_{3}\right)$} & & 0.0935 & & 0.0549 \\
\hline \multicolumn{2}{|l|}{ Wald $H \leq 0$ ( $p$-value $)$} & & 0.3399 & & 0.3986 \\
\hline \multicolumn{2}{|l|}{ Wald $H=1(p$-value $)$} & & 0.0000 & & 0.0000 \\
\hline \multicolumn{2}{|l|}{ Equilibrium test (ROE) ( $p$-value) } & 0.9875 & 0.1593 & 0.9875 & 0.1593 \\
\hline \multicolumn{2}{|l|}{$N$} & 318 & 318 & 318 & 318 \\
\hline \multicolumn{2}{|l|}{ Adjusted-R2 } & 0.990 & 0.991 & 0.996 & 0.996 \\
\hline
\end{tabular}

Table 3.7. The Impact of Merger on H-statistic of Clearing and Settlement Institutions

\begin{tabular}{|c|c|c|c|c|c|}
\hline \multirow{3}{*}{$\begin{array}{l}\text { Variable } \\
\ln \mathrm{AFR}\end{array}$} & \multirow{3}{*}{$\begin{array}{l}\text { Coefficients } \\
\beta\end{array}$} & \multicolumn{2}{|c|}{ Panel A: lnOPINCOM } & \multicolumn{2}{|c|}{ Panel B: $\ln T R$} \\
\hline & & & (2) & (1) & (2) \\
\hline & & 0.0306 & 0.0322 & 0.0508 & 0.0524 \\
\hline & & $(0.35)$ & $(0.37)$ & $(0.62)$ & $(0.64)$ \\
\hline \multirow[t]{2}{*}{$\operatorname{lnPPE}$} & $\gamma$ & $-0.152 *$ & $-0.154 *$ & $-0.180 * *$ & $-0.182 * *$ \\
\hline & & $(-1.68)$ & $(-1.68)$ & $(-2.06)$ & $(-2.06)$ \\
\hline \multirow[t]{2}{*}{$\ln P C E$} & $\zeta$ & 0.0429 & 0.0425 & 0.0428 & 0.0428 \\
\hline & & $(0.61)$ & $(0.59)$ & $(0.65)$ & $(0.63)$ \\
\hline \multirow[t]{2}{*}{ OI/OR } & $\psi$ & -0.152 & -0.149 & $0.162 *$ & $0.164 *$ \\
\hline & & $(-1.54)$ & $(-1.50)$ & $(1.70)$ & $(1.70)$ \\
\hline \multirow[t]{2}{*}{ Merger } & $\Pi_{4}$ & 0.306 & $1.155^{* *}$ & $0.347^{*}$ & $1.199 * *$ \\
\hline & & $(1.61)$ & $(2.37)$ & $(1.83)$ & $(2.45)$ \\
\hline \multirow[t]{2}{*}{$\ln \mathrm{AFR} *$ Merger } & $\beta_{4}$ & & -0.0334 & & -0.0353 \\
\hline & & & $(-0.92)$ & & $(-0.98)$ \\
\hline \multirow[t]{2}{*}{$\operatorname{lnPPE} *$ Merger } & $\gamma_{4}$ & & $0.206^{* *}$ & & $0.200 * *$ \\
\hline & & & $(2.05)$ & & (1.99) \\
\hline \multirow[t]{2}{*}{ lnPCE $*$ Merger } & $\zeta_{4}$ & & 0.0667 & & 0.0419 \\
\hline & & & $(0.60)$ & & $(0.39)$ \\
\hline \multirow[t]{2}{*}{$H O$-statistic $(\beta+\gamma+\zeta)$} & & -0.0785 & -0.0793 & -0.0864 & -0.0868 \\
\hline & & 91 & & & \\
\hline
\end{tabular}


Table 3.7. The Impact of Merger on H-statistic of Clearing and Settlement Institutions

\begin{tabular}{|c|c|c|c|c|}
\hline \multirow[t]{2}{*}{ Coefficients } & \multicolumn{2}{|c|}{ Panel A: $\ln$ OPINCOM } & \multicolumn{2}{|c|}{ Panel B: $\ln T R$} \\
\hline & & $(2)$ & $(1)$ & $(2)$ \\
\hline Wald $H O \leq 0$ ( $p$-value $)$ & 0.6913 & 0.6904 & 0.7123 & 0.7113 \\
\hline Wald $H O=1(p$-value $)$ & 0.0000 & 0.0000 & 0.0000 & 0.0000 \\
\hline$\beta_{4}+\gamma_{4}+\zeta_{4}$ & & 0.2393 & & 0.2066 \\
\hline Wald $\beta_{4}+\gamma_{4}+\zeta_{4} \leq 0$ ( $p$-value $)$ & & 0.0808 & & 0.1095 \\
\hline$H$-statistic $\left(\beta+\gamma+\zeta+\beta_{4}+\gamma_{4}+\zeta_{4}\right)$ & & 0.1600 & & 0.2066 \\
\hline Wald $H \leq 0$ ( $p$-value $)$ & & 0.2315 & & 0.2933 \\
\hline Wald $H=1$ ( $p$-value $)$ & & 0.0000 & & 0.0001 \\
\hline Equilibrium test (ROE) ( $p$-value) & 0.4077 & 0.0675 & 0.4077 & 0.0675 \\
\hline$N$ & 318 & 318 & 318 & 318 \\
\hline Adjusted-R2 & 0.986 & 0.986 & 0.994 & 0.994 \\
\hline
\end{tabular}

Note. The dependent variable lnOPINCOM represents log of operating income; lnTR represents log of total revenue. All regressions are OLS estimation. Dummy variables associated with clearing and settlement institutions and years are included. Heteroskedasticity robust $t$-values are reported in parentheses. Superscripts $* * *, * * *$ indicate significant level of $0.01,0.05$ and 0.10 , respectively. The $p$-values of the Wald tests are also provided.

Technological development: We also analyze whether competition in the clearing and settlement industry has increased with the fast development in information and communication technology. Table 3.8 indicates that the ICT ratio $_{\text {it }}$ is statistically significantly positively associated with $H$-statistic $\left(\beta_{5}+\gamma_{5}+\zeta_{5}\right.$ is positive). This provides support for the Hypothesis 3.5 that predicts positive relationship between competition and technological development.

Table 3.8. The Impact of ICT Ratio on H-statistic of Clearing and Settlement Institutions

\begin{tabular}{|c|c|c|c|c|c|}
\hline \multirow[t]{2}{*}{ Variable } & \multirow[t]{2}{*}{ Coefficients } & \multicolumn{2}{|c|}{ Panel A: InOPINCOM } & \multicolumn{2}{|c|}{ Panel B: InTR } \\
\hline & & & (2) & (1) & (2) \\
\hline \multirow[t]{2}{*}{$\ln A F R$} & $\beta$ & -0.0935 & $-0.391 * * *$ & -0.0588 & $-0.372 * * *$ \\
\hline & & $(-1.50)$ & $(-4.41)$ & $(-0.97)$ & $(-4.34)$ \\
\hline \multirow[t]{2}{*}{$\operatorname{lnPPE}$} & $\gamma$ & -0.107 & $-0.506^{* * *}$ & -0.119 & $-0.549 * * *$ \\
\hline & & $(-1.32)$ & $(-4.00)$ & $(-1.51)$ & $(-5.00)$ \\
\hline \multirow[t]{2}{*}{$\operatorname{lnPCE}$} & $\zeta$ & $-0.0860 * *$ & $-0.206^{* * *}$ & $-0.0682 *$ & $-0.178 * * *$ \\
\hline & & $(-2.28)$ & $(-5.01)$ & $(-1.74)$ & $(-4.19)$ \\
\hline \multirow[t]{2}{*}{ OI/OR } & $\psi$ & $-0.154 * *$ & $-0.228 * * *$ & $0.200 * *$ & $0.122 * *$ \\
\hline & & $(-2.21)$ & $(-5.63)$ & $(2.59)$ & $(2.45)$ \\
\hline \multirow[t]{2}{*}{ ICT ratio } & $\Pi_{5}$ & $0.0991 * * *$ & $0.209 * * *$ & $0.0896^{* *}$ & $0.211 * * *$ \\
\hline & & (2.81) & $(9.38)$ & $(2.45)$ & $(9.23)$ \\
\hline \multirow[t]{2}{*}{$\ln \mathrm{AFR} * \mathrm{ICT}$ ratio } & $\beta_{5}$ & & $0.0174 * * *$ & & $0.0177 * * *$ \\
\hline & & & (3.62) & & (3.76) \\
\hline \multirow[t]{2}{*}{$\operatorname{lnPPE} *$ ICT ratio } & $\gamma_{5}$ & & $0.0258 * * *$ & & $0.0281 * * *$ \\
\hline & & & (4.70) & & $(6.03)$ \\
\hline \multirow[t]{2}{*}{$\operatorname{lnPCE} *$ ICT ratio } & $\zeta_{5}$ & & $0.0125 * * *$ & & $0.0109 * * *$ \\
\hline & & & $(4.11)$ & & $(3.62)$ \\
\hline \multicolumn{2}{|l|}{$H 0$-statistic $(\beta+\gamma+\zeta)$} & -0.2865 & -1.1030 & -0.2460 & -1.0990 \\
\hline \multicolumn{2}{|c|}{ Wald $H O \leq 0(p$-value $)$} & 0.9781 & 0.9999 & 0.9534 & 0.9999 \\
\hline \multicolumn{2}{|c|}{ Wald $H O=1(p$-value $)$} & 0.0000 & 0.0000 & 0.0000 & 0.0000 \\
\hline \multicolumn{2}{|c|}{$\beta_{5}+\gamma_{5}+\zeta_{5}$} & & 0.0557 & & 0.0567 \\
\hline \multicolumn{2}{|c|}{ Wald $\beta_{5}+\gamma_{5}+\zeta_{5} \leq 0$ ( $p$-value $)$} & & 0.0000 & & 0.0000 \\
\hline \multicolumn{2}{|l|}{$H$-statistic } & & -1.0473 & & -1.0423 \\
\hline \multicolumn{2}{|c|}{ Wald $H \leq 0$ ( $p$-value $)$} & & 0.9999 & & 0.9999 \\
\hline \multicolumn{2}{|c|}{ Wald $H=1$ ( $p$-value $)$} & & 0.0000 & & 0.0000 \\
\hline \multicolumn{2}{|c|}{ Equilibrium test (ROE) ( $p$-value) } & 0.9234 & 0.8044 & 0.9234 & 0.8044 \\
\hline \multicolumn{2}{|c|}{$N$} & 209 & 209 & 209 & 209 \\
\hline \multicolumn{2}{|l|}{ Adjusted-R2 } & 0.990 & 0.993 & 0.996 & 0.997 \\
\hline
\end{tabular}

Note. The dependent variable lnOPINCOM represents log of operating income; lnTR represents log of total revenue. All regressions are OLS estimation. Dummy variables associated with clearing and settlement institutions and years are included. Heteroskedasticity robust $t$-values are reported in parentheses. Superscripts $* * *, * *, *$ indicate significant level of $0.01,0.05$ and 0.10 , respectively. The $p$-values of the Wald tests are also provided. 
Geographic location: We now analyze whether geographical location significantly affects competition between clearing and settlement institutions. In Table 3.9, we use the dummy variable USregion $_{\mathrm{i}}$ to compare the level of competition across the U.S. market and the European market. The null hypothesis that $\beta_{6}+\gamma_{6}+\zeta_{6} \leq 0$ is rejected at $1 \%$ level. Hence, $H$-statistic is higher in the U.S. market than in Europe. This is consistent with several studies showing that the costs of clearing and settlement services are higher in Europe than in the U.S. (Lannoo and Levin, 2002; NERA Economic Consulting, 2004).

Table 3.9. The Impact of Geographic Location (USregion) on H-statistic of Clearing and Settlement Institutions

\begin{tabular}{|c|c|c|c|c|c|}
\hline \multirow{3}{*}{$\begin{array}{l}\text { Variable } \\
\ln \mathrm{AFR}\end{array}$} & \multirow{3}{*}{$\begin{array}{l}\text { Coefficients } \\
\beta\end{array}$} & \multirow{2}{*}{\multicolumn{2}{|c|}{ Panel A: InOPINCOM }} & \multicolumn{2}{|c|}{ Panel B: $\ln T R$} \\
\hline & & & & (1) & (2) \\
\hline & & 0.0318 & 0.0225 & 0.0521 & 0.0425 \\
\hline & & $(0.36)$ & $(0.25)$ & $(0.63)$ & $(0.51)$ \\
\hline \multirow[t]{2}{*}{$\ln P P E$} & $\gamma$ & $-0.149 *$ & $-0.154^{*}$ & $-0.177 * *$ & $-0.181 * *$ \\
\hline & & $(-1.65)$ & $(-1.69)$ & $(-2.02)$ & $(-2.06)$ \\
\hline \multirow[t]{2}{*}{$\operatorname{lnPCE}$} & $\zeta$ & 0.0403 & 0.0401 & 0.0398 & 0.0404 \\
\hline & & $(0.58)$ & $(0.56)$ & $(0.60)$ & $(0.59)$ \\
\hline \multirow[t]{2}{*}{ OI/OR } & $\psi$ & -0.152 & -0.148 & $0.162 *$ & $0.166^{*}$ \\
\hline & & $(-1.53)$ & $(-1.51)$ & $(1.68)$ & $(1.75)$ \\
\hline \multirow[t]{2}{*}{ USregion } & $\Pi_{6}$ & $1.362^{*}$ & $3.565 * * *$ & $1.440^{*}$ & $3.652 * * *$ \\
\hline & & $(1.75)$ & (3.16) & $(1.78)$ & $(3.00)$ \\
\hline \multirow[t]{2}{*}{$\ln \mathrm{AFR} *$ USregion } & $\beta_{6}$ & & 0.195 & & 0.205 \\
\hline & & & $(0.83)$ & & $(0.80)$ \\
\hline \multirow[t]{2}{*}{$\operatorname{lnPPE} *$ USregion } & $\gamma_{6}$ & & $0.520 *$ & & $0.520 *$ \\
\hline & & & $(1.93)$ & & $(1.78)$ \\
\hline \multirow[t]{2}{*}{ lnPCE * USregion } & $\zeta_{6}$ & & -0.0487 & & -0.0794 \\
\hline & & & $(-0.44)$ & & $(-0.68)$ \\
\hline$H 0$-statistic $(\beta+\gamma+\zeta)$ & & -0.0769 & -0.0914 & -0.0851 & -0.0981 \\
\hline Wald $H O \leq 0$ ( $p$-value $)$ & & 0.6870 & 0.7121 & 0.7073 & 0.7310 \\
\hline Wald $H O=1$ ( $p$-value $)$ & & 0.0000 & 0.0000 & 0.0000 & 0.0000 \\
\hline$\beta_{6}+\gamma_{6}+\zeta_{6}$ & & & 0.6663 & & 0.6456 \\
\hline Wald $\beta_{6}+\gamma_{6}+\zeta_{6} \leq 0$ (p-value $)$ & & & 0.0029 & & 0.0078 \\
\hline$H$-statistic $\left(\beta+\gamma+\zeta+\beta_{6}+\gamma_{6}+\zeta_{6}\right)$ & & & 0.5749 & & 0.5475 \\
\hline Wald $H \leq 0$ (p-value $)$ & & & 0.0010 & & 0.0079 \\
\hline Wald $H=1$ (p-value) & & & 0.0226 & & 0.0421 \\
\hline Equilibrium test (ROE) ( $p$-value) & & 0.4073 & 0.3782 & 0.4073 & 0.3782 \\
\hline$N$ & & & & 318 & 318 \\
\hline Adjusted-R2 & & 0.986 & 0.986 & 0.994 & 0.994 \\
\hline
\end{tabular}

A caveat is in place. Bikker, Shaffer, and Spierdijk (2012) point to several weaknesses of the $H$-statistic. Bikker et al. (2012) prove that a negative $H$-statistic does not necessarily indicate monopoly even though the equilibrium test indicates a long-run equilibrium. They argue that $H$-statistic jointly measures competitive conduct and long-run structural equilibrium and, to evaluate its applicability, additional information is needed about costs, market equilibrium, and even market demand elasticity. In addition, $H$-statistic is not necessarily an ordinal function of the competitive conduct (see also Shaffer, 2004a). Therefore, we also analyze competition in the clearing and settlement industry by estimating the Lerner index and Boone indicator. 


\subsection{Factors Affecting the Lerner Index}

We now estimate Lerner index and regress them against a set of explanatory variables (see (3.9)).

Regression in column 1 of Table 3.10 only includes control variables. It shows that GDP growth is positively and significantly associated with the Lerner index. This indicates that competition between clearing and settlement institutions decreases when economy is growing. The number of clearing and settlement institutions in a given country is negatively and significantly associated with the Lerner index. This is consistent with the expectation that a higher number of institutions corresponds to higher competition in clearing and settlement. Inflation and interest rate are positively but insignificantly related to the Lerner index.

We now analyze which factors drive the Lerner index (columns 2 to 13 of Table 3.10). We find that a dummy variable, denoting the presence of the global financial crisis $\delta_{t}$, is (mostly) negatively and statistically significantly associated with the Lerner index. Hence, competition between clearing and settlement institutions is higher during the global financial crisis than in normal times. This is aligned with Hypothesis 3.1.

Dummy variable $\operatorname{ICSD}_{i}$ is negatively and highly statistically significantly (across all specifications) associated with the Lerner index. Negative relationship between $\operatorname{ICSD}_{\mathrm{i}}$ and the Lerner index indicates that international CSDs face higher competition than domestic CSDs. This confirms Hypothesis 3.2.

The Lerner index is negatively related to the size of a clearing and settlement institution. Hence, larger institutions are exposed to higher competition. This confirms Hypothesis 3.3. Dummy variable Merger is negatively but mostly insignificantly related to the Lerner index. This provides some but limited support for Hypothesis 3.4 that states that mergers between clearing and settlement institutions improve competition.

Dummy variable USregion $_{\mathrm{i}}$ is negatively and statistically significantly related to the Lerner index, indicating that competition between clearing and settlement institutions is higher in the U.S. market than in the European market. This provides additional support for Hypothesis 3.6.

Variables ICT ratio ${ }_{\text {it }}$ and time $t$ are negatively and significantly related to the Lerner index. We report regression with the ICT ratio it as a separate specification because an inclusion of the ICT ratio $_{\text {it }}$ significantly lowers the sample size. We also include separately variable time $t$ to prevent potential multicollinearity with crisis dummy $\delta_{t}$. We can conclude that technological development increases competition in clearing and settlement, confirming Hypothesis 3.5. 
Table 3.10. Estimation of Factors Affecting the Lerner Index

\begin{tabular}{|c|c|c|c|c|c|c|c|c|c|c|c|c|c|}
\hline Variable & (1) & (2) & (3) & (4) & (5) & (6) & (7) & (8) & (9) & (10) & (11) & (12) & (13) \\
\hline \multirow[t]{2}{*}{ GDP growth } & $0.0276^{* * * *}$ & $0.0228^{* *}$ & $0.0477 * * *$ & $0.0309 * * *$ & $0.0297 * * *$ & $0.0208 * *$ & -0.00433 & 0.0135 & $0.0453 * * *$ & $0.0430 * * *$ & $0.0405^{* * *}$ & 0.00127 & $0.0286^{* * *}$ \\
\hline & (2.92) & (2.03) & $(6.85)$ & (3.13) & (3.20) & (2.46) & $(-0.14)$ & (1.25) & $(4.25)$ & $(4.50)$ & (3.73) & $(0.02)$ & (2.58) \\
\hline \multirow[t]{2}{*}{ Inflation } & 0.00164 & -0.00350 & $-0.0243 * *$ & -0.0109 & 0.000494 & -0.00685 & 0.00982 & -0.0125 & -0.0219 & $-0.0329 * * *$ & -0.0196 & 0.0116 & -0.0117 \\
\hline & $(0.12)$ & $(-0.26)$ & $(-2.15)$ & $(-0.76)$ & $(0.04)$ & $(-0.59)$ & $(0.24)$ & $(-0.73)$ & $(-1.58)$ & $(-2.80)$ & $(-1.40)$ & $(0.21)$ & $(-0.75)$ \\
\hline \multirow[t]{2}{*}{ Interest Rate } & 0.00784 & -0.00304 & 0.00370 & -0.0163 & 0.00655 & 0.0130 & -0.00578 & -0.0121 & $-0.0179^{*}$ & -0.000155 & -0.0147 & -0.00627 & -0.0135 \\
\hline & $(0.82)$ & $(-0.30)$ & $(0.41)$ & $(-1.64)$ & $(0.67)$ & (1.48) & $(-0.23)$ & $(-1.00)$ & $(-1.65)$ & $(-0.02)$ & $(-1.35)$ & $(-0.16)$ & $(-1.09)$ \\
\hline \multirow{2}{*}{$\begin{array}{l}\text { Number of } \\
\text { Institutions }\end{array}$} & $-0.0316^{* *}$ & $-0.0265^{*}$ & 0.00873 & 0.00515 & $-0.0311^{* *}$ & $-0.0384 * * *$ & $0.218^{* * *}$ & -0.0181 & 0.0161 & -0.00275 & 0.00348 & $0.210^{* * * *}$ & -0.00103 \\
\hline & $(-2.09)$ & $(-1.75)$ & $(0.60)$ & $(0.33)$ & $(-2.08)$ & $(-2.87)$ & (5.99) & $(-1.04)$ & (1.03) & $(-0.18)$ & $(0.21)$ & (3.36) & $(-0.06)$ \\
\hline \multirow[t]{2}{*}{$\delta$} & & $-0.111^{*}$ & & & & & & & -0.0898 & -0.0877 & $-0.110^{*}$ & 0.118 & \\
\hline & & $(-1.71)$ & & & & & & & $(-1.35)$ & $(-1.43)$ & $(-1.67)$ & $(0.44)$ & \\
\hline \multirow[t]{2}{*}{ ICSD } & & & $-0.599 * * *$ & & & & & & $-0.441 * * *$ & $-0.440^{* * *}$ & $-0.336 * * *$ & & $-0.314 * * *$ \\
\hline & & & $(-10.81)$ & & & & & & $(-7.10)$ & $(-4.95)$ & $(-4.23)$ & & $(-3.86)$ \\
\hline \multirow[t]{2}{*}{ Size } & & & & $-0.0723 * * *$ & & & & & $-0.0371^{* *}$ & & $-0.0311^{*}$ & 0.0113 & -0.000476 \\
\hline & & & & $(-4.78)$ & & & & & $(-2.18)$ & & $(-1.81)$ & $(0.20)$ & $(-0.02)$ \\
\hline \multirow[t]{2}{*}{ Merger } & & & & & -0.0347 & & & & -0.0611 & $-0.171^{*}$ & -0.0742 & & -0.0681 \\
\hline & & & & & $(-0.52)$ & & & & $(-0.82)$ & $(-1.66)$ & $(-0.99)$ & & $(-0.90)$ \\
\hline \multirow[t]{2}{*}{ USregion } & & & & & & $-0.709 * * *$ & & & & $-0.280^{* * *}$ & $-0.238^{* *}$ & -0.0238 & $-0.202 *$ \\
\hline & & & & & & $(-12.05)$ & & & & $(-2.61)$ & $(-2.09)$ & $(-0.07)$ & $(-1.74)$ \\
\hline \multirow[t]{2}{*}{ ICT ratio } & & & & & & & $-0.0344 * * *$ & & & & & $-0.0353^{*}$ & \\
\hline & & & & & & & $(-3.76)$ & & & & & $(-1.65)$ & \\
\hline \multirow[t]{2}{*}{$t$} & & & & & & & & $-0.0167 * * *$ & & & & & $-0.0168 * * *$ \\
\hline & & & & & & & & $(-2.69)$ & & & & & $(-2.64)$ \\
\hline \multirow[t]{2}{*}{ Intercept } & $0.782 * * *$ & $0.854 * * *$ & $0.730 * * *$ & $1.284 * * *$ & $0.788^{* * *}$ & $0.876^{* * * *}$ & 0.303 & $1.082^{* * * *}$ & $1.033^{* * *}$ & $0.858 * * *$ & $1.041 * * *$ & 0.231 & $1.013^{* * *}$ \\
\hline & (7.83) & (8.03) & (7.76) & (7.78) & (7.95) & (9.95) & (1.10) & (6.09) & $(5.80)$ & $(8.23)$ & $(5.82)$ & $(0.41)$ & (5.17) \\
\hline$N$ & 207 & 207 & 207 & 207 & 207 & 207 & 124 & 207 & 207 & 207 & 207 & 124 & 207 \\
\hline Wald Chi2 & $14.15^{* * *}$ & $18.33^{* * * *}$ & $261.2 * * *$ & $46.65^{* * *}$ & $15.04 * * *$ & $161.5^{* * *}$ & $44.02 * * *$ & $13.59 * * *$ & $259.5^{* * *}$ & $414.3^{* * *}$ & $205.1^{* * *}$ & $36.74 * * *$ & $122.5^{* * *}$ \\
\hline
\end{tabular}

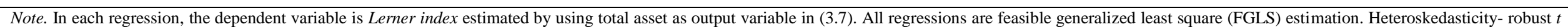
values are reported in parentheses. Superscripts $* * *, * *, *$ indicate significant level of $0.01,0.05$ and 0.10 , respectively. 


\subsection{Factors Affecting the Boone Indicator}

We now estimate the Boone indicator, which provides more directly the link between competition and efficiency of clearing and settlement institutions in our sample, and test the effect of several factors on the Boone indicator based on (3.10) and (3.11).

As expected, regression in column 1 of Table 3.11 shows that marginal cost is negatively and significantly associated with the profit of clearing and settlement institutions. We find that the interaction term of dummy variable the global financial crisis and marginal cost, $\delta_{\mathrm{t}} * \ln \mathrm{MC}_{\mathrm{it}}$, is negatively and statistically significantly associated with profit. This indicates that the negative Boone indicator during the financial crisis is lower than in normal times, and confirms that competition between clearing and settlement institutions is higher during the global financial crisis than in normal times. This is aligned with Hypothesis 3.1.

Interaction term between dummy variable $\operatorname{ICSD}_{\mathrm{i}}$ and marginal cost, $\operatorname{ICSD}_{\mathrm{i}} * \ln \mathrm{MC}_{\mathrm{it}}$, is negatively and statistically significantly related to profit. This indicates that the Boone indicator is lower for ICSDs than for CSDs. Consequently, ICSDs face higher competition than domestic CSDs. This confirms Hypothesis 3.2.

The interaction term between institution size and marginal cost, $\mathrm{Size}_{\mathrm{it}} * \ln \mathrm{MC}_{\mathrm{it}}$, is negatively and statistically significantly related to the profit of CSDs. Hence, larger institutions are exposed to higher competition. This confirms Hypothesis 3.3.

The interaction term between merger and marginal cost, Merger $_{i t} * \ln \mathrm{MC}_{\mathrm{it}}$, is negatively (but insignificantly) related to the profit. This provides some further support for Hypothesis 3.4 that states that the mergers between clearing and settlement institutions improve competition.

The interaction term between technological development and marginal cost, ICT ratio $\mathrm{it}^{*} \ln \mathrm{MC}_{\mathrm{it}}$ is negatively and significantly related to profit. This confirms that the ICT ratio ${ }_{\text {it }}$ is negatively and significantly related to the Boone indicator. Hence, technological development increases competition between clearing and settlement institutions, confirming Hypothesis 3.5.

Variable USregion ${ }_{\mathrm{i}} * \ln \mathrm{MC}_{\mathrm{it}}$ is negatively and statistically significantly related to profit, indicating that the Boone indicator is lower and that competition between clearing and settlement institutions is higher in the U.S. than in Europe. This confirms Hypothesis 3.6. 
Table 3.11. Estimation of Factors Affecting the Boone Indicator

\begin{tabular}{|c|c|c|c|c|c|c|c|c|c|c|c|c|c|}
\hline Variable & & (1) & (2) & (3) & (4) & (5) & (6) & (7) & (8) & (9) & (10) & (11) & (12) \\
\hline $\ln \mathrm{MC}$ & $\beta$ & $\begin{array}{c}-0.350 * * * \\
(-13.88)\end{array}$ & $\begin{array}{c}-0.333 * * * \\
(-12.88)\end{array}$ & $\begin{array}{c}-0.358 * * * \\
(-11.52)\end{array}$ & $\begin{array}{c}0.528 * * * \\
(6.05)\end{array}$ & $\begin{array}{c}-0.349 * * * \\
(-13.00)\end{array}$ & $\begin{array}{l}-0.0556 \\
(-0.68)\end{array}$ & $\begin{array}{c}-0.356 * * * \\
(-12.88)\end{array}$ & $\begin{array}{c}-0.289 * * * \\
(-8.50)\end{array}$ & $\begin{array}{c}0.831 * * * \\
(6.27)\end{array}$ & $\begin{array}{c}0.272 * * * \\
(3.54)\end{array}$ & $\begin{array}{c}0.830 * * * \\
(6.19)\end{array}$ & $\begin{array}{c}0.440 * * * \\
(4.95)\end{array}$ \\
\hline$\delta * \ln \mathrm{MC}$ & $\beta_{1}$ & & $\begin{array}{c}-0.0700 * * \\
(-2.10)\end{array}$ & & & & & & $\begin{array}{c}-0.110^{* * *} \\
(-2.98)\end{array}$ & & $\begin{array}{l}-0.0165 \\
(-0.45)\end{array}$ & $\begin{array}{l}-0.0303 \\
(-0.54)\end{array}$ & $\begin{array}{l}-0.0238 \\
(-0.65)\end{array}$ \\
\hline $\mathrm{ICSD} * \ln \mathrm{MC}$ & $\beta_{2}$ & & & $\begin{array}{c}-0.319 * * * \\
(-4.25)\end{array}$ & & & & & $\begin{array}{c}-0.323 * * * \\
(-4.37)\end{array}$ & $\begin{array}{l}-0.0956 \\
(-1.01)\end{array}$ & $\begin{array}{c}-0.238 * * * \\
(-3.14)\end{array}$ & & \\
\hline Size * $\ln \mathrm{MC}$ & $\beta_{3}$ & & & & $\begin{array}{c}-0.0816^{* * *} \\
(-9.91)\end{array}$ & & & & & $\begin{array}{c}-0.101 * * * \\
(-9.04)\end{array}$ & $\begin{array}{c}-0.0632 * * * \\
(-7.44)\end{array}$ & $\begin{array}{c}-0.0988 * * * \\
(-8.80)\end{array}$ & $\begin{array}{c}-0.0747 * * * \\
(-8.39)\end{array}$ \\
\hline Merger * $\ln \mathrm{MC}$ & $\beta_{4}$ & & & & & $\begin{array}{l}-0.0601 \\
(-0.54)\end{array}$ & & & $\begin{array}{l}-0.141 \\
(-1.13)\end{array}$ & & $\begin{array}{l}-0.0761 \\
(-0.49)\end{array}$ & & $\begin{array}{l}-0.0662 \\
(-0.42)\end{array}$ \\
\hline ICT ratio * $\ln \mathrm{MC}$ & $\beta_{5}$ & & & & & & $\begin{array}{c}-0.0168 * * * \\
(-5.42)\end{array}$ & & & $\begin{array}{c}-0.00263 \\
(-0.88)\end{array}$ & & $\begin{array}{c}-0.00378 \\
(-1.17)\end{array}$ & \\
\hline USregion * $\ln \mathrm{MC}$ & $\beta_{6}$ & & & & & & & $\begin{array}{c}-0.256^{* * *} \\
(-3.17)\end{array}$ & & & & $\begin{array}{c}-0.0773 \\
(-0.79)\end{array}$ & $\begin{array}{l}-0.129 \\
(-1.53)\end{array}$ \\
\hline Intercept & & $\begin{array}{c}1.434 * * * \\
(13.57)\end{array}$ & $\begin{array}{c}1.439 * * * \\
(15.44)\end{array}$ & $\begin{array}{c}1.297 * * * \\
(9.80)\end{array}$ & $\begin{array}{c}2.162^{* * *} \\
(14.16)\end{array}$ & $\begin{array}{c}1.435^{* * * *} \\
(12.99)\end{array}$ & $\begin{array}{c}1.239 * * * \\
(6.06)\end{array}$ & $\begin{array}{c}1.396^{* * * *} \\
(12.11)\end{array}$ & $\begin{array}{c}1.366^{* * * *} \\
(10.27)\end{array}$ & $\begin{array}{c}2.313^{* * *} \\
(9.11)\end{array}$ & $\begin{array}{c}1.583^{* * *} \\
(14.55)\end{array}$ & $\begin{array}{c}2.304^{* * * *} \\
(9.16)\end{array}$ & $\begin{array}{c}1.972 * * * \\
(13.39)\end{array}$ \\
\hline$N$ & & 286 & 286 & 286 & 286 & 286 & 186 & 286 & 286 & 186 & 286 & 186 & 286 \\
\hline Wald Chi2 & & $192.8 * * *$ & $213.7 * * *$ & $145.6^{* * *}$ & $169.4 * * *$ & $171.0^{* * *}$ & $152.0 * * *$ & $171.9^{* * *}$ & $127.4 * * *$ & $195.5^{* * *}$ & $219.3^{* * *}$ & $188.7 * * *$ & $172.3 * * *$ \\
\hline
\end{tabular}

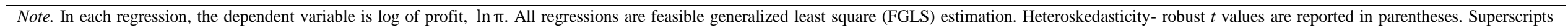
$* * *, * *, *$ indicate significant level of $0.01,0.05$ and 0.10 , respectively. 


\subsection{Conclusion}

Amid continued merger activities, competition is becoming a foremost issue in the currently still fragmented clearing and settlement industry. Using unbalanced annual data of 49 clearing and settlement institutions from 24 countries during 1989-2012, we analyze competition in clearing and settlement over times, across regions, and across different types of clearing and settlement institutions. We evaluate competition in the clearing and settlement industry using the structural and non-structural approach. We compute concentration indexes, the $H$-statistic of Panzar and Rosse $(1982,1987)$ model, the Lerner index, and Boone indicator.

We investigate the impact of the global financial crisis, institutional type, institutional size, mergers, technological development, and geographic location on the competition in clearing and settlement. Our findings suggest that although competition has increased over time, possibly due to the technological development, clearing and settlement institutions continue to operate in monopolistic markets. We confirm that larger size and mergers among clearing and settlement institutions lead to higher competition in clearing and settlement.

Our results support the view of Van Cayseele (2004) that contestable quasi-monopolies might be the most efficient industry structure among the feasible ones in clearing and settlement. The literature finds the presence of economies of scale in clearing and settlement (e.g., Van Cayseele and Wuyts, 2007). Clearing and settlement institutions can then exploit economies of scale through growth (either organic or through mergers or acquisitions). We refute the concerns that increased consolidation might hamper competition. In particular, we find that the creation of larger CSDs is associated with higher levels of competition. We also find that international CSDs face higher competition than domestic CSDs.

Our findings also suggest that competition between clearing and settlement institution in the U.S. market is higher than in the European market. This indicates that renewed initiative is necessary to enhance competition between clearing and settlement institutions in Europe. 


\section{GENERAL DISCUSSION AND CONCLUSION}

This chapter gives a general discussion on the main findings of the dissertation. The primary objective of this doctoral dissertation is to evaluate the role of financial derivatives, bank capital, and clearing and settlement services in normal times and during the global financial crisis. This chapter is structured as follows; first, it briefly summarizes the findings in each chapter of the dissertation and provides a systematic overview of them. Second, it discusses the main overarching theoretical and empirical contributions. The final part briefly concludes the dissertation.

\section{Summary of the Main Findings}

Building upon the extended four-factor model, Chapter 1 analyzes the relationship between the use of financial derivatives and systematic risk exposures of U.S. bank holding companies. The results confirm that a BHC's use of financial derivatives is associated with its higher exposure towards systematic interest rate risk, exchange rate risk, and credit risk (i.e., nondiversifiable risk exposures that investors cannot trade away on the financial markets). Interestingly, the positive relationship between financial derivatives and systematic risk exposure seems stronger for large BHCs than for small BHCs. Derivatives held for trading and derivatives held for hedging purposes are both positively and significantly related to BHCs' systematic risk exposures (in the case of interest rate derivatives, exchange rate derivatives, and credit derivatives). In the global financial crisis, the relationships between interest rate derivatives and exchange rate derivatives and systematic risk exposures became stronger than in normal time, and the positive relationship between credit derivatives and systematic credit risk became less pronounced.

Chapter 2 examines whether, and how, different types of bank capital affect bank lending. The impact of the size and several other dimensions that may influence the decline in credit growth during the financial crisis are also analyzed. The results indicate a positive effect of the tier 1 capital ratio on bank loan growth during the global financial crisis. The effect seems to be more pronounced for small banks and for banks in non-OECD and BRIC countries. Customer deposits also positively affected bank lending during the global financial crisis. Furthermore, the tier 2 capital ratio and interbank deposits positively affect loan growth in normal times and interbank deposits negatively affected bank lending during the global financial crisis. Whereas tier 1 capital and customer deposits acted as a stable source of funding during the global financial crisis, tier 2 capital and interbank deposits spur bank lending during normal times, but did not do so during the global financial crisis. During normal times, a bank lends more if the tier 1 capital ratio of competing banks is high. However, during the global financial crisis, a bank lent more if the tier 1 capital ratio of competing banks was low. Government ownership helped banks to better sustain credit growth during the global financial crisis. This effect was statistically significant only in non-OECD and BRIC countries, but not in OECD countries. 
Chapter 3 aims to analyze the competitive landscape in the clearing and settlement industry. Specifically, this chapter employs the Panzar and Rosse model (1982, 1987), Lerner index (1934), and Boone indicator $(2001,2008)$ to examine the competitive conditions in the clearing and settlement industry, and to test how competition is affected by several factors. The empirical results suggest the existence of monopoly equilibrium in the clearing and settlement industry. The Panzar Rosse model, Boone indicator, and Lerner index confirm the following conclusions: during the global financial crisis, the level of competition between clearing and settlement institutions is higher than in normal times. International CSDs face higher competition than CSDs in the local markets. The evidence also indicates that competition increases continuously over time, possibly due to technological development and implementation of new clearing and settlement systems. The results reveal that competition increases with the size of the clearing and settlement institutions and after mergers and acquisitions between clearing and settlement institutions. The findings also suggest that competition between clearing and settlement institutions is higher in the U.S. market than in the European market. This indicates that renewed initiative is necessary to enhance competition between clearing and settlement institutions in Europe.

Table 4.1 summarizes the research questions and main findings of each chapter, and presents the research methodologies we employed and the contribution to the existing literature. ${ }^{60}$

\section{Overarching Theoretical and Empirical Contributions}

An important methodological contribution of Chapter 1 of this dissertation is the extended Fama and French (1992) model and disentanglement of systematic (i.e., undiversifiable) risk into three components - systematic interest rate, exchange rate, and credit risk - at the same time. This allows us to jointly analyze the impact of interest rate derivatives, exchange rate derivatives, and credit derivatives on the corresponding systematic risks. The results show that financial derivatives are positively and significantly related to the systematic risk exposures of BHCs. Higher use of interest rate derivatives, exchange rate derivatives, and credit derivatives corresponds to greater systematic interest rate risk, exchange rate risk, and credit risk. Systematic risks are positively related to derivatives used for hedging purposes and to derivatives used for trading purposes.

Several factors affect the relationship between financial derivatives and systematic risks of BHCs. Financial derivatives expose large BHCs to higher level of systematic risk compared to small BHCs. High capital reinforces the positive relationship between financial derivatives for trading and systematic risks, but weakens the positive relationship between financial derivatives for hedging and systematic risk. In the global financial crisis, the relationship between interest rate derivatives and exchange rate derivatives and systematic risk exposures became stronger than in normal time, and the positive relationship between credit derivatives and systematic credit risk became less pronounced.

${ }^{60}$ In the Appendix D Table D.1, we summarize the main findings in terms of the hypotheses in each chapter. 
Table 4.1. Summary of Main Findings and Contributions in Chapters 1, 2, and 3.

\begin{tabular}{|c|c|c|c|c|c|}
\hline Chapter (Title) & Sample & Main Data Source & Methodology & Main Findings & Contribution \\
\hline $\begin{array}{l}\text { Chapter 1: The Use } \\
\text { of Financial } \\
\text { Derivatives and } \\
\text { Risks of U.S. Bank } \\
\text { Holding Companies }\end{array}$ & $\begin{array}{l}\text { 1997-2012, } \\
\text { BHCs }\end{array}$ & $\begin{array}{l}\text { FR Y-9C; } \\
\text { CRSP; } \\
\text { Federal Reserve } \\
\text { Board of Governors }\end{array}$ & $\begin{array}{l}\text { Fixed effect } \\
\text { model; } \\
\text { IV model; } \\
\text { GMM model }\end{array}$ & $\begin{array}{l}\text { Financial derivatives are positively and significantly related to } \\
\text { systematic risks of BHCs. } \\
\text { Derivatives held for trading and derivatives held for hedging } \\
\text { purposes are both positively and significantly related to BHCs' } \\
\text { systematic risk exposures. } \\
\text { In the global financial crisis, the relationship between interest rate } \\
\text { derivatives and exchange rate derivatives and systematic risk } \\
\text { exposures became stronger than in normal time, and the positive } \\
\text { relationship between credit derivatives and systematic credit risk } \\
\text { became less pronounced. }\end{array}$ & $\begin{array}{l}\text { The use of extended } \\
\text { four-factor model to } \\
\text { obtain systematic risks } \\
\text { factors, including } \\
\text { systematic credit risk; } \\
\text { Differentiation of } \\
\text { financial derivatives used } \\
\text { for hedging and for } \\
\text { trading purposes. }\end{array}$ \\
\hline 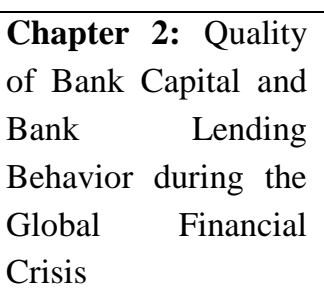 & $\begin{array}{l}2000-2010, \\
\text { Worldwide } \\
\text { banks }\end{array}$ & BankScope & $\begin{array}{l}\text { Fixed effect } \\
\text { model; } \\
\text { IV model; } \\
\text { GMM model }\end{array}$ & $\begin{array}{l}\text { High-quality bank funding sources (i.e., tier } 1 \text { bank capital and } \\
\text { retail deposits) and prevalent government backing were crucial for } \\
\text { continuous bank lending during the crisis period. } \\
\text { Higher use of tier } 2 \text { capital and interbank deposits could be } \\
\text { important for increased lending during a normal period; this did not } \\
\text { support lending activities during the financial crisis. }\end{array}$ & $\begin{array}{l}\text { Estimate separately the } \\
\text { impact of capital structure } \\
\text { (Tier } 1 \text { and Tier } 2 \text { capital } \\
\text { ratio) on bank lending in } \\
\text { normal times and during } \\
\text { the global crisis. }\end{array}$ \\
\hline $\begin{array}{l}\text { Chapter } 3 \text { : } \\
\text { Competition in the } \\
\text { Clearing and } \\
\text { Settlement Industry }\end{array}$ & $\begin{array}{l}\text { 1989-2012, } \\
\text { Clearing and } \\
\text { settlement } \\
\text { institutions }\end{array}$ & $\begin{array}{l}\text { BankScope; } \\
\text { Annual Reports; } \\
\text { World Bank. }\end{array}$ & $\begin{array}{l}\text { Panzar-Rosse } \\
\text { model; } \\
\text { the Lerner } \\
\text { Index; } \\
\text { the Boone } \\
\text { Indicator }\end{array}$ & $\begin{array}{l}\text { Clearing and settlement industry operates under the monopoly } \\
\text { equilibrium. } \\
\text { During the global financial crisis, the level of competition between } \\
\text { clearing and settlement institutions is higher than in normal times. } \\
\text { International CSDs face higher competition than CSDs in the local } \\
\text { market. } \\
\text { Competition increases continuously over time, possibly due to the } \\
\text { technological development and implementation of new clearing and } \\
\text { settlement systems. } \\
\text { Competition increases with the size of clearing and settlement } \\
\text { institutions and after mergers and acquisitions between clearing and } \\
\text { settlement institutions. } \\
\text { Competition between clearing and settlement institution in the U.S. } \\
\text { market is significantly higher than in the European market. }\end{array}$ & $\begin{array}{l}\text { This is the first } \\
\text { comprehensive study on } \\
\text { the competitive conditions } \\
\text { in the clearing and } \\
\text { settlement industry; } \\
\text { The analysis of factors } \\
\text { that affect competition in } \\
\text { clearing and settlement. }\end{array}$ \\
\hline
\end{tabular}


The contribution of Chapter 2 of this dissertation is to distinguish between tier 1 and tier 2 capital, and customer and interbank deposits as bank funding sources and to evaluate their relationship to bank lending. The results indicate that the higher quality of the bank funding side (i.e., a high tier 1 bank capital ratio, and high proportion of customer deposits) better supports bank lending during the crisis times. In contrast, tier 2 capital does not provide adequate support for bank lending during a financial crisis.

The empirical analysis in Chapter 2 reveals that the factors including different types of deposits and ownership are important in shaping the bank lending behavior in normal times and during the financial crisis. Interbank deposits negatively affected bank lending during the global financial crisis. Banks tried to compensate for this by turning to more stable funding sources, such as retail deposits (European Central Bank, 2011). Meanwhile, customer deposits were positively related to loan growth during the global financial crisis, which indicates customer (retail) deposits were sticky and acted as a stable source of funding even during the global financial crisis. In addition, banks were largely supported by the governments to overcome refinancing difficulties during the crisis, and this points to the benefits of government ownership in mitigating the credit crunch.

The contribution of Chapter 3 in this study is three-fold and incorporates important contributions to the field of competition literature, with special focus on clearing and settlement industry. While previous studies focus on the economies of scale, cost and revenue efficiency, and technology developments in the clearing and settlement industry. For example, Schmiedel, Malkamaki, and Tarkka (2006) focus on the factors of economies of scales and technological development. Van Cayseele and Wuyts (2007) find that economies of scale exist in European clearing and settlement; however, they are exhausted far below the size of the entire European market. This dissertation analyzes the competitive conditions in the clearing and settlement industry. In particular, it employs PR model, the Lerner index, and Boone indicator, to examine the competition of clearing and settlement institutions. The PR model, Lerner index, and Boone indicator have been extensively used to analyze the nature of competition in banking systems, but had never been applied to the clearing and settlement institutions. Finally, this analysis compares competition in the clearing and settlement industry across the U.S. and Europe.

\section{Conclusion}

This dissertation analyzes the role of financial derivatives, bank capital (and other bank funding sources), and clearing and settlement activities in normal times and during the global financial crisis. It analyzes the relationship between the use of financial derivatives and risks. It investigates the relation between the quality of bank capital and bank lending growth. In addition, this dissertation examines the competitive conditions in the clearing and settlement industry and tests the impact of several factors on competitive conditions in the clearing and settlement industry.

The methodologies employed in this analysis fill in an important gap in the previous literature, 
and provide insights and advancements that will help management communities and policy makers to better understand the performance of financial institutions. From a managerial point of view, financial institutions can improve their performance if they better understand how financial derivatives contribute to risk exposures, how bank capital structure decision could affect the credit growth, and how different factors affect the competitive conditions in clearing and settlement. 


\section{REFERENCE LIST}

Acharya, V. V., Mehran, H., \& Thakor, A. (2010). Caught between Scylla and Charybdis? Regulating Bank Leverage When There Is Rent-Seeking and Risk-Shifting. Federal Reserve Bank of New York. New York.

Acharya, V. V., \& Yorulmazer, T. (2008). Information Contagion and Bank Herding. Journal of Money, Credit and Banking, 40(1), 215-231.

Adams, R. M., Bauer, P. W., \& Sickles, R. (2004). Scale Economies, Scope Economies, and Technical Change in Federal Reserve Payment Processing. Journal of Money, Credit and Banking, 36(5), 943-958.

Admati, A., DeMarzo, P., Hellwig, M., \& Pfleiderer, P. (2012). Debt Overhang and Capital Regulation. Rock Center for Corporate Governance at Stanford University Working Paper No. 114, MPI Collective Goods Preprint, No. 2012/5.

Admati, A. R., DeMarzo, P. M., Hellwig, M., \& Pfleiderer, P. (2010). Fallacies, Irrelevant Facts, and Myths in the Discussion of Capital Regulation: Why Bank Equity Is Not Expensive. Max Planck Inst. for Research on Collective Goods.

Ahmed, A. S., Kilic, E., \& Lobo, G. J. (2011). Effects of SFAS 133 on the Risk Relevance of Accounting Measures of Banks' Derivative Exposures. The Accounting Review, 86(3), 769-804.

Al-Muharrami, S., Matthews, K., \& Khabari, Y. (2006). Market Structure and Competitive Conditions in the Arab GCC Banking System. Journal of Banking \& Finance, 30(12), 3487-3501.

Allen, F., Carletti, E., \& Marquez, R. (2011). Credit Market Competition and Capital Regulation. Review of Financial Studies, 24(4), 983-1018.

Allen, F., Hryckiewicz, A., Kowalewski, O., \& Tümer-Alkan, G. (2012). Transmission of Bank Liquidity Shocks in Loan and Deposit Markets: The Role of Interbank Borrowing and Market Monitoring. Wharton Financial Institution Center Working Paper.

Altunbas, Y., Gambacorta, L., \& Marques-Ibanez, D. (2012). Do Bank Characteristics Influence the Effect of Monetary Policy on Bank Risk? Economics Letters, 117(1), 220-222.

Altunbas, Y., Manganelli, S., \& Marques-Ibanez, D. (2011). Bank Risk During the Financial Crisis: Do Business Models Matter? ECB Working Paper Series 1394. European Central Bank.

Ammer, J., Vega, C., \& Wongswan, J. (2010). International Transmission of US Monetary Policy Shocks: Evidence from Stock Prices. Journal of Money, Credit and Banking, 42(s1), 179-198.

Anderson, T. W., \& Rubin, H. (1949). Estimation of the Parameters of a Single Equation in a Complete System of Stochastic Equations. The Annals of Mathematical Statistics, 20(1), 46-63.

Angelini, P., \& Cetorelli, N. (2003). The Effects of Regulatory Reform on Competition in the Banking Industry. Journal of Money, Credit and Banking, 35(5), 663-684.

Appelbaum, E. (1979). Testing Price Taking Behavior. Journal of Econometrics, 9(3), 283-294.

Arellano, M., \& Bond, S. (1991). Some Tests of Specification for Panel Data: Monte Carlo 
Evidence and an Application to Employment Equations. The Review of Economic Studies, 58(2), 277-297.

Ashcraft, A. B. (2008a). Are Bank Holding Companies a Source of Strength to Their Banking Subsidiaries? Journal of Money, Credit and Banking, 40(2/3), 273-294.

Ashcraft, A. B. (2008b). Does the Market Discipline Banks? New Evidence from Regulatory Capital Mix. Journal of Financial Intermediation, 17(4), 543-561.

Atkinson, T., Luttrell, D., \& Rosenblum, H. (2013). How Bad Was It? The Costs and Consequences of the 2007-09 Financial Crisis. Federal Reserve Bank of Dallas Staff Papers No.20.

Ayuso, J., Pérez, D., \& Saurina, J. (2004). Are Capital Buffers Pro-Cyclical?: Evidence from Spanish Panel Data. Journal of Financial Intermediation, 13(2), 249-264.

Bain, J. S. (1951). Relation of Profit Rate to Industry Concentration: American Manufacturing, 1936-1940. The Quarterly Journal of Economics, 65(3), 293-324.

Bajo, E., Bigelli, M., Hillier, D., \& Petracci, B. (2009). The Determinants of Regulatory Compliance: An Analysis of Insider Trading Disclosures in Italy. Journal of Business Ethics, 90(3), 331-343.

Barrell, R., Davis, E. P., Fic, T., \& Karim, D. (2011). Bank Capital Composition, Regulation and Risk Taking. NIESR and Brunel University London.

Barth, J. R., Caprio, G., \& Levine, R. (2004). Bank Regulation and Supervision: What Works Best? Journal of Financial Intermediation, 13(2), 205-248.

Basel Committee on Banking Supervision. (2010). Basel III: A Global Regulatory Framework for More Resilient Banks and Banking Systems. http://www.bis.org/press/p100912.htm

Bauer, P. W., \& Ferrier, G. D. (1996). Scale Economies, Cost Efficiencies, and Technological Change in Federal Reserve Payments Processing. Journal of Money, Credit and Banking, 28(4), 1004-1039.

Bauer, W., \& Ryser, M. (2004). Risk Management Strategies for Banks. Journal of Banking \& Finance, 28(2), 331-352.

Baum, C. F. (2006). An Introduction to Modern Econometrics Using Stata: Stata Corp.

Baum, C. F., Schaffer, M. E., \& Stillman, S. (2007). Enhanced Routines for Instrumental Variables/GMM Estimation and Testing. Stata Journal, 7(4), 465-506.

Beck, T., De Jonghe, O., \& Schepens, G. (2013). Bank Competition and Stability: Cross-Country Heterogeneity. Journal of Financial Intermediation, 22(2), 218-244.

Beck, T., Demirguc-Kunt, A., \& Levine, R. (2006). Bank Concentration, Competition, and Crises: First Results. Journal of Banking \& Finance, 30(5), 1581-1603.

Beijnen, C., \& Bolt, W. (2009). Size Matters: Economies of Scale in European Payments Processing. Journal of Banking \& Finance, 33(2), 203-210.

Berger, A. N. (2006). Potential Competitive Effects of Basel II on Banks in SME Credit Markets in the United States. Journal of Financial Services Research, 29(1), 5-36.

Berger, A. N., \& Bonaccorsi di Patti, E. (2006). Capital Structure and Firm Performance: A New Approach to Testing Agency Theory and an Application to the Banking Industry. Journal of Banking \& Finance, 30(4), 1065-1102.

Berger, A. N., \& Bouwman, C. H. (2009). Bank Liquidity Creation. Review of Financial Studies, 22(9), 3779-3837. 
Berger, A. N., \& Bouwman, C. H. (2013). How Does Capital Affect Bank Performance During Financial Crises? Journal of Financial Economics, 109(1), 146-176.

Berger, A. N., Bouwman, C., Kick, T., \& Schaeck, K. (2012). Bank Risk Taking and Liquidity Creation Following Regulatory Interventions and Capital Support. Working paper, July 2012.

Berger, A. N., DeYoung, R., Flannery, M. J., Lee, D., \& Öztekin, Ö. (2008). How Do Large Banking Organizations Manage Their Capital Ratios? Journal of Financial Services Research, 34(2/3), 123-149.

Berger, A. N., Hasan, I., \& Klapper, L. F. (2004). Further Evidence on the Link between Finance and Growth: An International Analysis of Community Banking and Economic Performance. Journal of Financial Services Research, 25(2), 169-202.

Berger, A. N., Hasan, I., \& Zhou, M. (2009). Bank Ownership and Efficiency in China: What Will Happen in the World's Largest Nation? Journal of Banking \& Finance, 33(1), 113-130.

Berger, A. N., Klapper, L. F., \& Turk-Ariss, R. (2009). Bank Competition and Financial Stability. Journal of Financial Services Research, 35(2), 99-118.

Berger, A. N., \& Udell, G. F. (1994). Did Risk-Based Capital Allocate Bank Credit and Cause a "Credit Crunch" in the United States? Journal of Money, Credit and Banking, 26(3), 585-628.

Berlin, M., \& Mester, L. J. (1999). Deposits and Relationship Lending. Review of Financial Studies, 12(3), 579-607.

Bernanke, B. S. (1990). Clearing and Settlement During the Crash. Review of Financial Studies, 3(1), 133-151.

Berrospide, J., \& Edge, R. (2010). The Effects of Bank Capital on Lending: What Do We Know, and What Does It Mean? International Journal of Central Banking, 6(34), 1-50.

Białkowski, J., Etebari, A., \& Wisniewski, T. P. (2012). Fast Profits: Investor Sentiment and Stock Returns During Ramadan. Journal of Banking \& Finance, 36(3), 835-845.

Bikker, J. A. (2004). Competition and Efficiency in a Unified European Banking Market. Cheltenham, UK: Edward Elgar Publishing.

Bikker, J. A., \& Bos, J. W. B. (2005). Trends in Competition and Profitability in the Banking Industry: A Basic Framework. Vienna: SUERF.

Bikker, J. A., \& Groeneveld, J. M. (2000). Competition and Concentration in the EU Banking Industry. Kredit und Kapital, 33(1), 62-98.

Bikker, J. A., \& Haaf, K. (2002). Competition, Concentration and Their Relationship: An Empirical Analysis of the Banking Industry. Journal of Banking \& Finance, 26(11), 2191-2214.

Bikker, J. A., Shaffer, S., \& Spierdijk, L. (2012). Assessing Competition with the Panzar-Rosse Model: The Role of Scale, Costs, and Equilibrium. Review of Economics and Statistics, 94(4), 1025-1044.

Bikker, J. A., Spierdijk, L., \& Finnie, P. (2006). The Impact of Bank Size on Market Power. DNB Working Papers No.120. De Nederlandsche Bank. Amsterdam.

Bikker, J. A., Spierdijk, L., \& Finnie, P. (2007). Misspecifiation of the Panzar-Rosse Model: Assessing Competition in the Banking Industry. DNB Working Papers No.114. De 
Nederlandsche Bank. Amsterdam.

Billmeier, A., \& Nannicini, T. (2013). Assessing Economic Liberalization Episodes: A Synthetic Control Approach. Review of Economics and Statistics, 95(3), 983-1001.

Bolt, W., \& Humphrey, D. (2007). Payment Network Scale Economies, SEPA, and Cash Replacement. Review of Network Economics, 6(4), 453-473.

Bonin, J. P., Hasan, I., \& Wachtel, P. (2005a). Bank Performance, Efficiency and Ownership in Transition Countries. Journal of Banking \& Finance, 29(1), 31-53.

Bonin, J. P., Hasan, I., \& Wachtel, P. (2005b). Privatization Matters: Bank Efficiency in Transition Countries. Journal of Banking \& Finance, 29(8), 2155-2178.

Boone, J. (2001). Intensity of Competition and the Incentive to Innovate. International Journal of Industrial Organization, 19(5), 705-726.

Boone, J. (2008). A New Way to Measure Competition. The Economic Journal, 118(531), $1245-1261$.

Boot, A. W. A. (2011). Banking at the Crossroads: How to Deal with Marketability and Complexity? Review of Development Finance, 1(3/4), 167-183.

Boot, A. W. A. (2014). Financial Sector in Flux. Journal of Money, Credit and Banking, 46(s1), 129-135.

Boot, A. W. A., \& Marinč, M. (2008). The Evolving Landscape of Banking. Industrial and Corporate Change, 17(6), 1173-1203.

Boot, A. W., \& Ratnovski, L. (2013). Banking and Trading. IMF Working Paper WP12/238. International Monetary Fund.

Boot, A. W. A., \& Thakor, A. V. (1991). Off-Balance Sheet Liabilities, Deposit Insurance and Capital Regulation. Journal of Banking \& Finance, 15(4), 825-846.

Boot, A. W. A., \& Thakor, A. V. (2010). The Accelerating Integration of Banks and Markets and Its Implications for Regulation. In Berger, A., Molyneux, P. and Wilson, J. (Eds.), The Oxford Handbook of Banking (pp. 58-90). Oxford: Oxford University Press.

Bredin, D., \& Hyde, S. (2011). Investigating Sources of Unanticipated Exposure in Industry Stock Returns. Journal of Banking \& Finance, 35(5), 1128-1142.

Brei, M., Gambacorta, L., \& von Peter, G. (2013). Rescue Packages and Bank Lending. Journal of Banking \& Finance, 37(2), 490-505.

Bresnahan, T. F. (1982). The Oligopoly Solution Concept Is Identified. Economics Letters, 10(1), 87-92.

Brewer, E., Kaufman, G. G., \& Wall, L. D. (2008). Bank Capital Ratios across Countries: Why Do They Vary? Journal of Financial Services Research, 34(2/3), 177-201.

Byoun, S. (2008). How and When Do Firms Adjust Their Capital Structures toward Targets? The Journal of Finance, 63(6), 3069-3096.

Calomiris, C. W., \& Kahn, C. M. (1991). The Role of Demandable Debt in Structuring Optimal Banking Arrangements. The American Economic Review, 81(3), 497-513.

Carbó, S., Humphrey, D., Maudos, J., \& Molyneux, P. (2009). Cross-Country Comparisons of Competition and Pricing Power in European Banking. Journal of International Money and Finance, 28(1), 115-134.

Carlson, M., Shan, H., \& Warusawitharana, M. (2013). Capital Ratios and Bank Lending: A Matched Bank Approach. Journal of Financial Intermediation, 22(4), 663-687. 
Carter, D. A., \& Sinkey, J. F. (1998). The Use of Interest Rate Derivatives by End-Users: The Case of Large Community Banks. Journal of Financial Services Research, 14(1), 17-34.

Casu, B., \& Girardone, C. (2006). Bank Competition, Concentration and Efficiency in the Single European Market. The Manchester School, 74(4), 441-468.

Cetorelli, N., \& Goldberg, L. S. (2009). Globalized Banks: Lending to Emerging Markets in the Crisis. Federal Reserve Bank of New York Staff Report No. 377.

Chamberlain, G. (1982). Multivariate Regression Models for Panel Data. Journal of Econometrics, 18(1), 5-46.

Chaudhry, M., Christie-David, R., Koch, T., \& Reichert, A. (2000). The Risk of Foreign Currency Contingent Claims at US Commercial Banks. Journal of Banking \& Finance, 24(9), 1399-1417.

Cherbonnier, F., \& Rochet, J.-C. (2010). Vertical Integration and Regulation in the Securities Settlement Industry. TSE Working Paper.

Choi, J. J., \& Elyasiani, E. (1997). Derivative Exposure and the Interest Rate and Exchange Rate Risks of US Banks. Journal of Financial Services Research, 12(2), 267-286.

Choi, J. J., Elyasiani, E., \& Kopecky, K. J. (1992). The Sensitivity of Bank Stock Returns to Market, Interest and Exchange Rate Risks. Journal of Banking \& Finance, 16(5), 983-1004.

Choi, J. J., \& Jiang, C. (2009). Does Multinationality Matter? Implications of Operational Hedging for the Exchange Risk Exposure. Journal of Banking \& Finance, 33(11), 1973-1982.

Chue, T. K., \& Cook, D. (2008). Emerging Market Exchange Rate Exposure. Journal of Banking \& Finance, 32(7), 1349-1362.

Cipollini, A., \& Fiordelisi, F. (2012). Economic Value, Competition and Financial Distress in the European Banking System. Journal of Banking \& Finance, 36(11), 3101-3109.

Claessens, S., \& Laeven, L. (2004). What Drives Bank Competition? Some International Evidence. Journal of Money, Credit and Banking, 36(3), 563-583.

Coccorese, P. (1998). Assessing the Competitive Conditions in the Italian Banking System: Some Empirical Evidence. Banca Nazionale del Lavoro Quarterly Review, 51(205), 171-191.

Coccorese, P. (2004). Banking Competition and Macroeconomic Conditions: A Disaggregate Analysis. Journal of International Financial Markets, Institutions and Money, 14(3), 203-219.

Coccorese, P. (2009). Market Power in Local Banking Monopolies. Journal of Banking \& Finance, 33(7), 1196-1210.

Collender, R. N., \& Shaffer, S. (2003). Local Bank Office Ownership, Deposit Control, Market Structure, and Economic Growth. Journal of Banking \& Finance, 27(1), 27-57.

Columba, F., Gambacorta, L., \& Mistrulli, P. E. (2009). The Effects of Mutual Guarantee Consortia on the Quality of Bank Lending. MPRA Paper 17052. Bank of Italy.

Columba, F., Gambacorta, L., \& Mistrulli, P. E. (2010). Mutual Guarantee Institutions and Small Business Finance. Journal of Financial Stability, 6(1), 45-54.

Cornett, M. M., McNutt, J. J., Strahan, P. E., \& Tehranian, H. (2011). Liquidity Risk Management and Credit Supply in the Financial Crisis. Journal of Financial Economics, 101(2), 297-312. 
Cragg, J. G., \& Donald, S. G. (1993). Testing Identifiability and Specification in Instrumental Variable Models. Econometric Theory, 9(2), 222-240.

Cyree, K. B., Huang, P., \& Lindley, J. T. (2012). The Economic Consequences of Banks' Derivatives Use in Good Times and Bad Times. Journal of Financial Services Research, 41(3), 121-144.

Dahiya, S., Puri, M., \& Saunders, A. (2003). Bank Borrowers and Loan Sales: New Evidence on the Uniqueness of Bank Loans. The Journal of Business, 76(4), 563-582.

De Bandt, O., \& Davis, E. P. (2000). Competition, Contestability and Market Structure in European Banking Sectors on the Eve of EMU. Journal of Banking \& Finance, 24(6), 1045-1066.

De Carvalho, C. (2004). Cross-Border Securities Clearing and Settlement Infrastructure in the European Union as a Prerequisite to Financial Markets Integration: Challenges and Perspectives. HWWA Discussion Paper No. 287.

De Haas, R., Korniyenko, Y., Loukoianova, E., \& Pivovarsky, A. (2012). Foreign Banks and the Vienna Initiative: Turning Sinners into Saints? IMF Working WP/12/117. International Monetary Fund.

De Haas, R., \& Van Lelyveld, I. (2010). Internal Capital Markets and Lending by Multinational Bank Subsidiaries. Journal of Financial Intermediation, 19(1), 1-25.

Delis, M. D., Staikouras, K. C., \& Varlagas, P. T. (2008). On the Measurement of Market Power in the Banking Industry. Journal of Business Finance \& Accounting, 35(7/8), 1023-1047.

Dell'Ariccia, G., \& Marquez, R. (2006). Lending Booms and Lending Standards. The Journal of Finance, 61(5), 2511-2546.

Demirgüç-Kunt, A., Detragiache, E., \& Merrouche, O. (2013). Bank Capital: Lessons from the Financial Crisis. Journal of Money, Credit and Banking, 45(6), 1147-1164.

Demirgüç-Kunt, A., Kane, E. J., Karacaovali, B., \& Laeven, L. (2008). Deposit Insurance around the World: A Comprehensive Database. In Demirgüç-Kunt, A., Kane, E. J. and Laeven, L. (Eds.), Deposit Insurance around the World: Issues of Design and Implementation (pp. 363-382). Cambridge, MA: The MIT Press.

Demsetz, H. (1968). The Cost of Transacting. The Quarterly Journal of Economics, 82(1), 33-53.

Demsetz, R. S., Saidenberg, M. R., \& Strahan, P. E. (1996). Banks with Something to Lose: The Disciplinary Role of Franchise Value. Economic Policy Review, 2(2), 1-14.

Dewally, M., \& Shao, Y. (2012). Financial Derivatives, Opacity, and Crash Risk: Evidence from Large US Banks. Journal of Financial Stability, 9(4), 565-577.

Diamond, D. (1984). Financial Intermediation and Delegated Monitoring. The Review of Economic Studies, 51(3), 393-414.

Doidge, C., Griffin, J., \& Williamson, R. (2006). Measuring the Economic Importance of Exchange Rate Exposure. Journal of Empirical Finance, 13(4), 550-576.

Drakos, K., \& Konstantinou, P. (2005). Competition and Contestability in Transition Banking: An Empirical Analysis. South-Eastern Europe Journal of Economics, 2, 183-209.

Driscoll, J. C., \& Kraay, A. C. (1998). Consistent Covariance Matrix Estimation with Spatially Dependent Panel Data. Review of Economics and Statistics, 80(4), 549-560.

Duffee, G. R., \& Zhou, C. (2001). Credit Derivatives in Banking: Useful Tools for Managing 
Risk? Journal of Monetary Economics, 48(1), 25-54.

Elyasiani, E., \& Mansur, I. (1998). Sensitivity of the Bank Stock Returns Distribution to Changes in the Level and Volatility of Interest Rate: A GARCH-M Model. Journal of Banking \& Finance, 22(5), 535-563.

Elyasiani, E., \& Mansur, I. (2004). Bank Stock Return Sensitivities to the Long-Term and Short-Term Interest Rates: A Multivariate GARCH Approach. Managerial Finance, 30(9), $32-55$.

European Central Bank. (2007). T2S Economic Feasibility Study. https://www.ecb.int/press/pr/date/2007/html/pr070308_2.en.html.

European Central Bank. (2008). T2S Economic Impact Assessment. https://www.ecb.int/paym/t2s/about/keydocs/html/index.en.html.

European Central Bank. (2011). Financial Stability Review. http://www.ecb.int/pub/pdf/other/financialstabilityreview201106en.pdf.

Faff, R. W., Hodgson, A., \& Kremmer, M. L. (2005). An Investigation of the Impact of Interest Rates and Interest Rate Volatility on Australian Financial Sector Stock Return Distributions. Journal of Business Finance \& Accounting, 32 (5 - 6), 1001-1031.

Fama, E. F., \& French, K. R. (1992). The Cross-Section of Expected Stock Returns. The Journal of Finance, 47(2), 427-465.

Financial Accounting Standards Board (FASB). (1998). Accounting for Derivative Instruments and Hedging Activities. Statement of Financial Accounting Standards No. 133. Stamford, CT: FASB.

Fiordelisi, F., \& Marques-Ibanez, D. (2013). Is Bank Default Risk Systematic? Journal of Banking \& Finance, 37(6), 2000-2010.

Flannery, M., \& James, C. (1984). The Effect of Interest Rate Changes on the Common Stock Returns of Financial Institutions. The Journal of Finance, 39(4), 1141-1153.

Flannery, M. J., \& Rangan, K. P. (2008). What Caused the Bank Capital Build-up of the 1990s? Review of Finance, 12(2), 391-429.

Foos, D., Norden, L., \& Weber, M. (2010). Loan Growth and Riskiness of Banks. Journal of Banking \& Finance, 34(12), 2929-2940.

Francis, B. B., Hasan, I., \& Hunter, D. M. (2008). Can Hedging Tell the Full Story? Reconciling Differences in United States Aggregate-and Industry-Level Exchange Rate Risk Premium. Journal of Financial Economics, 90(2), 169-196.

Froot, K. A., Scharfstein, D. S., \& Stein, J. C. (1993). Risk Managements Coordinating Corporate Investment and Financing Policies. The Journal of Finance, 48(5), 1629-1658.

Fu, X. M., Lin, Y. R., \& Molyneux, P. (2014). Bank Competition and Financial Stability in Asia Pacific. Journal of Banking \& Finance, 38, 64-77.

Géczy, C., Minton, B., \& Schrand, C. (1997). Why Firms Use Currency Derivatives. The Journal of Finance, 52(4), 1323-1354.

Günalp, B., \& Çelik, T. (2006). Competition in the Turkish Banking Industry. Applied Economics, $38(11), 1335-1342$.

Gambacorta, L., \& Marques - Ibanez, D. (2011). The Bank Lending Channel: Lessons from the Crisis. Economic Policy, 26(66), 135-182.

Gambacorta, L., \& Mistrulli, P. E. (2004). Does Bank Capital Affect Lending Behavior? Journal 
of Financial Intermediation, 13(4), 436-457.

Gehrig, T., \& Stenbacka, R. (2007). Information Sharing and Lending Market Competition with Switching Costs and Poaching. European Economic Review, 51(1), 77-99.

Gelos, R. G., \& Roldós, J. (2004). Consolidation and Market Structure in Emerging Market Banking Systems. Emerging Markets Review, 5(1), 39-59.

Giddy, I., Saunders, A., \& Walter, I. (1996). Alternative Models for Clearance and Settlement: The Case of the Single European Capital Market. Journal of Money, Credit and Banking, 28(4), 986-1000.

Giovannini Group. (2002). Cross-Border Clearing and Settlement Arrangements in the European Union. Directorate-General for Economic and Financial Affairs, European Commission.

Giovannini Group. (2003). Second Report on EU Clearing and Settlement Arrangements. Directorate-General for Economic and Financial Affairs, European Commission.

Gischer, H., \& Stiele, M. (2009). Competition Tests with a Non-Structural Model: The PanzarRosse Method Applied to Germany's Savings Banks. German Economic Review, 10(1), 50-70.

Goddard, J., \& Wilson, J. O. (2009). Competition in Banking: A Disequilibrium Approach. Journal of Banking \& Finance, 33(12), 2282-2292.

Goodhart, C. A. E. (2013). Ratio Controls Need Reconsideration. Journal of Financial Stability, 9(3), 445-450.

Gorton, G., \& Rosen, R. (1995). Banks and Derivatives. NBER Macroeconomics Annual.

Greenspan, A. (1999). Financial Derivatives. Before the Futures Industry Association, Boca Raton, Florida. March 19, 1999

Gropp, R., Hakenes, H., \& Schnabel, I. (2010). Competition, Risk-Shifting, and Public Bail-out Policies. Review of Financial Studies, 24(6), 2084.

Gropp, R., \& Heider, F. (2010). The Determinants of Bank Capital Structure. Review of Finance, 14(4), 587-622.

Guay, W., \& Kothari, S. P. (2003). How Much Do Firms Hedge with Derivatives? Journal of Financial Economics, 70(3), 423-461.

Gunther, J. W., \& Siems, T. F. (2002). The Likelihood and Extent of Banks' Involvement with Interest Rate Derivatives as End Users. Research in Finance, 19, 125-142.

Hall, A. R., \& Peixe, F. P. M. (2000). Data Mining and the Selection of Instruments. Journal of Economic Methodology, 7(2), 265-277.

Hall, A. R., Rudebusch, G. D., \& Wilcox, D. W. (1996). Judging Instrument Relevance in Instrumental Variables Estimation. International economic review, 37(2), 283-298.

Hancock, D., Humphrey, D. B., \& Wilcox, J. A. (1999). Cost Reductions in Electronic Payments: The Roles of Consolidation, Economies of Scale, and Technical Change. Journal of Banking \& Finance, 23(2), 391-421.

Hancock, D., \& Wilcox, J. A. (1998). The "Credit Crunch" and the Availability of Credit to Small Business. Journal of Banking \& Finance, 22(6), 983-1014.

Hart, O., \& Zingales, L. (2011). A New Capital Regulation for Large Financial Institutions. American Law and Economics Review, 13(2), 453-490.

Hasan, I., \& Malkamäki, M. (2001). Are Expansions Cost Effective for Stock Exchanges? A Global Perspective. Journal of Banking \& Finance, 25(12), 2339-2366. 
Hasan, I., Malkamäki, M., \& Schmiedel, H. (2003). Technology, Automation, and Productivity of Stock Exchanges: International Evidence. Journal of Banking \& Finance, 27(9), 1743-1773.

Hasan, I., \& Schmiedel, H. (2004). Networks and Equity Market Integration: European Evidence. International Review of Financial Analysis, 13(5), 601-619.

Hasan, I., Schmiedel, H., \& Song, L. (2012a). Growth Strategies and Value Creation: What Works Best for Stock Exchanges? Financial Review, 47(3), 469-499.

Hasan, I., Schmiedel, H., \& Song, L. (2012b). Returns to Retail Banking and Payments. Journal of Financial Services Research, 41(3), 163-195.

Hasan, I., Siddique, A., \& and Sun, X. (2015). Monitoring the "Invisible" Hand of Market Discipline: Capital Adequacy Revisited. Journal of Banking \& Finance, 50, 475-492.

Hau, H., Langfield, S., \& Marques - Ibanez, D. (2013). Bank Ratings: What Determines Their Quality? Economic Policy, 28(74), 289-333.

Hellmann, T. F., Murdock, K. C., \& Stiglitz, J. E. (2000). Liberalization, Moral Hazard in Banking, and Prudential Regulation: Are Capital Requirements Enough? American Economic Review, 90(1), 147-165.

Hempell, H. (2002). Testing for Competition among German Banks. Economic Research Centre of the Deutsche Bundesbank discussion paper No.04/02.

Hirtle, B. (1997). Derivatives, Portfolio Composition, and Bank Holding Company Interest Rate Risk Exposure. Journal of Financial Services Research, 12(2), 243-266.

Hirtle, B. (2009). Credit Derivatives and Bank Credit Supply. Journal of Financial Intermediation, 18(2), 125-150.

Holthausen, C., \& Tapking, J. (2007). Raising Rival's Costs in the Securities Settlement Industry. Journal of Financial Intermediation, 16(1), 91-116.

Hondroyiannis, G., Lolos, S., \& Papapetrou, E. (1999). Assessing Competitive Conditions in the Greek Banking System. Journal of International Financial Markets, Institutions and Money, 9(4), 377-391.

Hunter, W., \& Timme, S. (1986). Technical Change, Organizational Form, and the Structure of Bank Production. Journal of Money, Credit and Banking, 18(2), 152-166.

Hutson, E., \& Stevenson, S. (2009). Openness, Hedging Incentives and Foreign Exchange Exposure: A Firm-Level Multi-Country Study. Journal of International Business Studies, 41(1), 105-122.

Independent Commission on Banking. (2011). Final Report Recommendations. London.

Ivashina, V., \& Scharfstein, D. (2010). Bank Lending During the Financial Crisis of 2008. Journal of Financial Economics, 97(3), 319-338.

Iwata, G. (1974). Measurement of Conjectural Variations in Oligopoly. Econometrica: Journal of the Econometric Society, 947-966.

Jiang, G., Wong, J., Tang, N., \& Sze, A. (2004). Banking Sector Competition in Hong KongMeasurement and Evolution over Time. Hong Kong Monetary Authority (Vol. 30, pp. 40).

Jiménez, G., Ongena, S., Peydró, J. L., \& Saurina Salas, J. (2012). Credit Supply: Identifying Balance-Sheet Channels with Loan Applications and Granted Loans. American Economic Review, 102(5), 2301-2326.

Jokipii, T., \& Milne, A. (2008). The Cyclical Behaviour of European Bank Capital Buffers. 
Journal of Banking \& Finance, 32(8), 1440-1451.

Juranek, S., \& Walz, U. (2010). Vertical Integration, Competition, and Financial Exchanges: Is there Grain in the Silo? CFS Working Paper No. 2010/22.

Kauko, K. (2007). Interlinking Securities Settlement Systems: A Strategic Commitment? Journal of Banking \& Finance, 31(10), 2962-2977.

Kashyap, A., Rajan, R., \& Stein, J. (2008). Rethinking Capital Regulation. Federal Reserve Bank of Kansas City Symposium on Maintaining Stability in a Changing Financial System. Kansas City.

Kashyap, A. K., \& Stein, J. C. (1997). What Do a Million Banks Have to Say About the Transmission of Monetary Policy? NBER Working Paper No. 6056. National Bureau of Economic Research.

Keeley, M. C. (1990). Deposit Insurance, Risk, and Market Power in Banking. The American Economic Review, 80(5), 1183-1200.

Kleibergen, F., \& Paap, R. (2006). Generalized Reduced Rank Tests Using the Singular Value Decomposition. Journal of Econometrics, 133(1), 97-126.

Knieps, G. (2006). Competition in the Post-Trade Markets: A Network Economic Analysis of the Securities Business. Journal of Industry, Competition and Trade, 6(1), 45-60.

Koetter, M., Kolari, J. W., \& Spierdijk, L. (2012). Enjoying the Quiet Life under Deregulation? Evidence from Adjusted Lerner Indices for US Banks. Review of Economics and Statistics, 94(2), 462-480.

Köppl, T. V., \& Monnet, C. (2007). Guess What: It's the Settlements! Vertical Integration as a Barrier to Efficient Exchange Consolidation. Journal of Banking \& Finance, 31(10), 3013-3033.

Kumbhakar, S. C., \& Lovell, C. K. (2000). Stochastic Frontier Analysis. Cambridge: Cambridge University Press.

Lannoo, K., \& Levin, M. (2002). The Securities Settlement Industry in the EU: Structure, Costs and the Way Forward. CEPS Reports in Finance and Banking No. 26. CEPS.

Lau, L. J. (1982). On Identifying the Degree of Competitiveness from Industry Price and Output Data. Economics Letters, 10(1), 93-99.

Lee, M. H., \& Nagano, M. (2008). Market Competition before and after Bank Merger Wave: A Comparative Study of Korea and Japan. Pacific Economic Review, 13(5), 604-619.

Lee, S., \& Lee, J. (2005). Bank Consolidation and Bank Competition: An Empirical Analysis of the Korean Banking Industry. Bank of Korea Economic Paper 8.

Lensink, R., Meesters, A., \& Naaborg, I. (2008). Bank Efficiency and Foreign Ownership: Do Good Institutions Matter? Journal of Banking \& Finance, 32(5), 834-844.

Lerner, A. P. (1934). The Concept of Monopoly and the Measurement of Monopoly Power. The Review of Economic Studies, 1(3), 157-175.

Levine, R., Loayza, N., \& Beck, T. (2000). Financial Intermediation and Growth: Causality and Causes. Journal of Monetary Economics, 46(1), 31-77.

Lim, S., Sum, V., \& Khun, C. (2012). Interest Rate Sensitivity of Stock Returns: Effects across the Maturity Profile and Direction of Interest Rate Changes. International Research Journal of Applied Finance, 3(8), 1151-1162.

Liu, H., Molyneux, P., \& Wilson, J. O. (2013a). Competition and Stability in European Banking: 
A Regional Analysis. The Manchester School, 81(2), 176-201.

Liu, H., Molyneux, P., \& Wilson, J. O. (2013b). Competition in Banking: Measurement and Interpretation. In Bell, A., Brooks, C. and Prokopczuk, M. (Eds.), Handbook of Research Methods and Applications in Empirical Finance (pp. 197-215). Cheltenham, UK: Edward Elgar Publishing.

MaCurdy, T. E. (1982). The Use of Time Series Processes to Model the Error Structure of Earnings in a Longitudinal Data Analysis. Journal of Econometrics, 18(1), 83-114.

Mamatzakis, E., Staikouras, C., \& Koutsomanoli-Fillipaki, N. (2005). Competition and Concentration in the Banking Sector of the South Eastern European Region. Emerging Markets Review, 6(2), 192-209.

Marinč, M. (2013). Banks and Information Technology: Marketability vs. Relationships. Electronic Commerce Research, 13(1), 71-101.

Mason, E. S. (1939). Price and Production Policies of Large-Scale Enterprise. The American Economic Review, 29(1), 61-74.

Matthews, K., Murinde, V., \& Zhao, T. (2007). Competitive Conditions among the Major British Banks. Journal of Banking \& Finance, 31(7), 2025-2042.

Maudos, J., \& Solís, L. (2011). Deregulation, Liberalization and Consolidation of the Mexican Banking System: Effects on Competition. Journal of International Money and Finance, 30(2), 337-353.

Mayordomo, S., Rodriguez-Moreno, M., \& Peña, J. I. (2014). Derivatives Holdings and Systemic Risk in the US Banking Sector. Journal of Banking \& Finance, 45, 84-104.

Mehran, H., \& Thakor, A. (2011). Bank Capital and Value in the Cross-Section. Review of Financial Studies, 24(4), 1019-1067.

Memmel, C., \& Raupach, P. (2010). How Do Banks Adjust Their Capital Ratios? Journal of Financial Intermediation, 19(4), 509-528.

Mercier, F., \& Sauer, S. (2013). Optimal CSD Reshaping Towards T2S. European Central Bank Working Paper Series No. 1549.

Mester, L. J. (1987). Multiple Market Contact between Savings and Loans. Journal of Money, Credit and Banking, 19(4), 538-549.

Mian, S. (1996). Evidence on Corporate Hedging Policy. Journal of Financial and Quantitative Analysis, 31(03), 419-439.

Milne, A. (2007a). Competition and the Rationalization of European Securities Clearing and Settlement. In Mayes, D. and Wood, G. E. (Eds.), The Structure of Financial Regulation (pp. 370-409). New York: Routledge.

Milne, A. (2007b). The Industrial Organization of Post-Trade Clearing and Settlement. Journal of Banking \& Finance, 31(10), 2945-2961.

Minton, B. A., Stulz, R., \& Williamson, R. (2009). How Much Do Banks Use Credit Derivatives to Hedge Loans? Journal of Financial Services Research, 35(1), 1-31.

Mkrtchyan, A. (2005). The Evolution of Competition in Banking in a Transition Economy: An Application of the Panzar-Rosse Model to Armenia. The European Journal of Comparative Economics, 2(1), 67-82.

Molyneux, P., Lloyd-Williams, D. M., \& Thornton, J. (1994). Competitive Conditions in European Banking. Journal of Banking \& Finance, 18(3), 445-459. 
Molyneux, P., Thornton, J., \& Llyod-Williams, D. M. (1996). Competition and Market Contestability in Japanese Commercial Banking. Journal of Economics and Business, $48(1), 33-45$.

Morrison, A. D. (2005). Credit Derivatives, Disintermediation, and Investment Decisions. The Journal of Business, 78(2), 621-648.

Murjan, W., \& Ruza, C. (2002). The Competitive Nature of the Arab Middle Eastern Banking Markets. International Advances in Economic Research, 8(4), 267-274.

Nance, D., Smith Jr, C., \& Smithson, C. (1993). On the Determinants of Corporate Hedging. The Journal of Finance, 48(1), 267-284.

Nathan, A., \& Neave, E. H. (1989). Competition and Contestability in Canada's Financial System: Empirical Results. Canadian Journal of Economics, 19(3), 576-594.

NERA Economic Consulting. (2004). The Direct Costs of Clearing and Settlement: An EU-US Comparison. City Research Series.

Nielsson, U. (2009). Stock Exchange Merger and Liquidity: The Case of Euronext. Journal of Financial Markets, 12(2), 229-267.

Norden, L., Buston, C. S., \& Wagner, W. (2011). Banks' Use of Credit Derivatives and the Pricing of Loans: What Is the Channel and Does It Persist under Adverse Economic Conditions. Erasmus University Rotterdam and Tilburg University. Working Paper.

OECD. (2011). OECD Factbook 2011: Economic, Environmental and Social Statistics. OECD.

Office of the Comptroller of the Currency. (2012). OCC's Quarterly Report on Bank Trading and $\begin{array}{llll}\text { Derivatives } & \text { Activities } & \text { Fourth }\end{array}$ http://www.occ.gov/topics/capital-markets/financial-markets/trading/derivatives/dq411.p $\underline{\mathrm{df}}$

Olivero, M. P., Li, Y., \& Jeon, B. N. (2011). Competition in Banking and the Lending Channel: Evidence from Bank-Level Data in Asia and Latin America. Journal of Banking \& Finance, 35(3), 560-571.

Panzar, J. C., \& Rosse, J. N. (1982). Structure, Conduct, and Comparative Statistics. Economics Discussion Paper No.248. Bell Telephone Laboratories, Inc.

Panzar, J. C., \& Rosse, J. N. (1987). Testing for "Monopoly" Equilibrium. The Journal of Industrial Economics, 35(4), 443-456.

Pirrong, C. (2007). The Industrial Organization of Execution, Clearing and Settlement in Financial Markets. CFS Working Paper, No. 2008/43.

Puri, M., Rocholl, J., \& Steffen, S. (2009). The Impact of the US Financial Crisis on Global Retail Lending. Fuqua School of Business Duke University, mimeo.

Purnanandam, A. (2007). Interest Rate Derivatives at Commercial Banks: An Empirical Investigation. Journal of Monetary Economics, 54(6), 1769-1808.

Rajan, R. G. (1994). Why Bank Credit Policies Fluctuate: A Theory and Some Evidence. The Quarterly Journal of Economics, 109(2), 399-441.

Rampini, A. A., \& Viswanathan, S. (2010). Collateral, Risk Management, and the Distribution of Debt Capacity. The Journal of Finance, 65(6), 2293-2322.

Reichert, A., \& Shyu, Y. (2003). Derivative Activities and the Risk of International Banks: A Market Index and VaR Approach. International Review of Financial Analysis, 12(5), 489-511. 
Rime, B. (2001). Capital Requirements and Bank Behaviour: Empirical Evidence for Switzerland. Journal of Banking \& Finance, 25(4), 789-805.

Rochet, J.-C. (2005). The Welfare Effects of Vertical Integration in the Securities Clearing and Settlement Industry. IDEI, Toulouse University, mimeo.

Roodman, D. (2009). How to Do xtabond2: An Introduction to Difference and System GMM in Stata. The Stata Journal 9(1), 86-136.

Rosati, S., \& Secola, S. (2006). Explaining Cross-Border Large-Value Payment Flows: Evidence from TARGET and EURO1 Data. Journal of Banking \& Finance, 30(6), 1753-1782.

Saporoschenko, A. (2002). The Sensitivity of Japanese Bank Stock Returns to Economic Factors: An Examination of Asset/Liability Differences and Main Bank Status. Global Finance Journal, 13(2), 253-270.

Schaeck, K., Cihak, M., \& Wolfe, S. (2009). Are Competitive Banking Systems More Stable? Journal of Money, Credit and Banking, 41(4), 711-734.

Schaper, T. (2008). Trends in European Cross-Border Securities SettlementTARGET2-Securities and the Code of Conduct. In Veit, D., Kundisch, D., Weitzel, T. and Rajola, F. (Eds.), Enterprise Applications and Services in the Finance Industry (Vol. 4, pp. 50-65). Springer.

Schaper, T., \& Chlistalla, M. (2010). The Impact of Information Technology on European Post-Trading. In Nelson, M. L., Shaw, M. and Strader, T., J. (Eds.), Sustainable E-Business Management (pp. 48-62). Springer.

Schmiedel, H. (2001). Technological Development and Concentration of Stock Exchanges in Europe. Bank of Finland Discussion Paper Series, 21/2001. Helsinki.

Schmiedel, H., Malkamäki, M., \& Tarkka, J. (2006). Economies of Scale and Technological Development in Securities Depository and Settlement Systems. Journal of Banking \& Finance, 30(6), 1783-1806.

Schmiedel, H., \& Schönenberger, A. (2005). Integration of Securities Market Infrastructures in the Euro Area. European Central Bank Working Paper Series No. 33. European Central Bank.

Serifsoy, B., \& Weiß, M. (2007). Settling for Efficiency-a Framework for the European Securities Transaction Industry. Journal of Banking \& Finance, 31(10), 3034-3057.

Shaffer, S. (1982). A Non-Structural Test for Competition in Financial Markets. Paper presented at the Proceedings of a Conference on Bank Structure and Competition.

Shaffer, S. (1983a). Non-Structural Measures of Competition: Toward a Synthesis of Alternatives. Economics Letters, 12(3), 349-353.

Shaffer, S. (1983b). The Rosse-Panzar Statistic and the Lerner Index in the Short Run. Economics Letters, 11(1), 175-178.

Shaffer, S. (1993). A Test of Competition in Canadian Banking. Journal of Money, Credit and Banking, 25(1), 49-61.

Shaffer, S. (2002). Conduct in a Banking Monopoly. Review of Industrial Organization, 20(3), 221-238.

Shaffer, S. (2004a). Comment on "What Drives Bank Competition? Some International Evidence" by Stijn Claessens and Luc Laeven. Journal of Money, Credit and Banking, $36(3), 585-592$. 
Shaffer, S. (2004b). Patterns of Competition in Banking. Journal of Economics and Business, 56(4), 287-313.

Shaffer, S. (1999). The Competitive Impact of Disclosure Requirements in the Credit Card Industry. Journal of Regulatory Economics, 15(2), 183-198.

Shen, C.-H., Hasan, I., \& Lin, C.-Y. (2014). The Government's Role in Government-Owned Banks. Journal of Financial Services Research, 45(3), 307-340.

Shen, C.-H., \& Lin, C.-Y. (2012). Why Government Banks Underperform: A Political Interference View. Journal of Financial Intermediation, 21(2), 181-202.

Smith, C., \& Stulz, R. (1985). The Determinants of Firms' Hedging Policies. Journal of Financial and Quantitative Analysis, 20(04), 391-405.

Song, F., \& Thakor, A. V. (2007). Relationship Banking, Fragility, and the Asset-Liability Matching Problem. Review of Financial Studies, 20(6), 2129-2177.

Staikouras, C. K., \& Koutsomanoli - Fillipaki, A. (2006). Competition and Concentration in the New European Banking Landscape. European Financial Management, 12(3), 443-482.

Standard and Poor's. (2011). Industry Risk for Investment Banking Is Generally Higher Than for Other Financial Institutions. January 6.

Stavarek, D., \& Repkova, I. (2011). Estimation of the Competitive Conditions in the Czech Banking Sector. Acta Universitatis Agriculturae et Silviculturae Mendelianae Brunensis, 59(2), 299-305.

Stulz, R. M. (1996). Rethinking Risk Management. Journal of Applied Corporate Finance, 9(3), 8-25.

Stulz, R. M. (2003). Risk Management \& Derivatives. Cincinnati, Ohio: Southwestern Publishing.

Tapking, J., \& Yang, J. (2006). Horizontal and Vertical Integration and Securities Trading and Settlement. Journal of Money, Credit and Banking, 38(7), 1-16.

Thakor, A. V. (2012a). Incentives to Innovate and Financial Crises. Journal of Financial Economics, 103(1), 130-148.

Thakor, A. V. (2012b). The Economic Consequences of the Volcker Rule. Report by the US Chamber's Center for Capital Market Competitiveness.

Thakor, A. V. (2014). The Financial Crisis of 2007-09: Why Did It Happen and What Did We Learn? Available at SSRN: http://dx.doi.org/10.2139/ssrn.2372449.

The Financial Stability Board. (2010). Implementing OTC Derivatives Market Reforms. Financial Stability Board.

Trichet, J. C. (2007). Some Reflections on the Development of Credit Derivatives. Keynote address at the 22nd Annual General Meeting of the International Swaps and Derivatives Association (ISDA), Boston.

Tufano, P. (1989). Financial Innovation and First-Mover Advantages. Journal of Financial Economics, 25(2), 213-240.

Turk-Ariss, R. (2010). On the Implications of Market Power in Banking: Evidence from Developing Countries. Journal of Banking \& Finance, 34(4), 765-775.

Van Cayseele, P. (2004). Competition and the Organisation of the Clearing and Settlement Industry. CES, KU Leuven Discussion Paper.

Van Cayseele, P., \& Wuyts, C. (2007). Cost Efficiency in the European Securities Settlement and 
Depository Industry. Journal of Banking \& Finance, 31(10), 3058-3079.

VanHoose, D. (2007). Theories of Bank Behavior under Capital Regulation. Journal of Banking \& Finance, 31(12), 3680-3697.

Vesala, J. (1995). Testing for Competition in Banking: Behavioral Evidence from Finland. Bank of Finland Studies Working Paper E:1. Bank of Finland. Helsinki.

Viale, A. M., Kolari, J. W., \& Fraser, D. R. (2009). Common Risk Factors in Bank Stocks. Journal of Banking \& Finance, 33(3), 464-472.

White, H. (1980). A Heteroskedasticity-Consistent Covariance Matrix Estimator and a Direct Test for Heteroskedasticity. Econometrica, 48, 817-838.

World Bank. (2008). Bank Regulation and Supervision.

http://econ.worldbank.org/WBSITE/EXTERNAL/EXTDEC/EXTRESEARCH/0,, content MDK:20345037 pagePK:64214825 piPK:64214943 theSitePK:469382,00.html\#Surve y_III.

Yeyati, E. L., \& Micco, A. (2003). Banking Competition in Latin America. Paper presented at the Latin American Competition Forum.

Yildirim, H. S., \& Philippatos, G. C. (2007). Restructuring, Consolidation and Competition in Latin American Banking Markets. Journal of Banking \& Finance, 31(3), 629-639.

Yong, H. H. A., Faff, R. W., \& Chalmers, K. (2009). Derivative Activities and Asia-Pacific Banks' Interest Rate and Exchange Rate Exposures. Journal of International Financial Markets, Institutions and Money, 19(1), 16-32. 
APPENDICES 



\section{LIST OF APPENDICES}

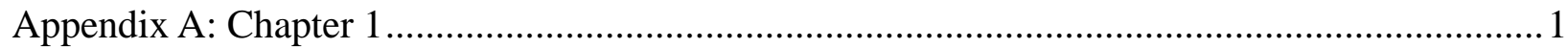

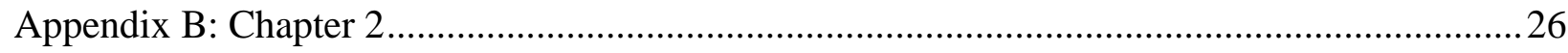

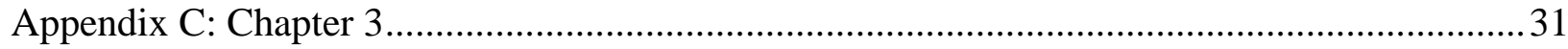

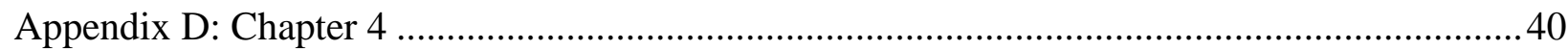

Appendix E: Summary in Slovenian language / Daljši povzetek disertacije v slovenskem ......... 43 



\section{Appendix A: Chapter 1}

Table A.1. Diagnostic Tests of Instruments Used in IV Regression in Table 1.8

Total Sample

Large BHCs

Small BHCs

\section{Panel A: Interest Rate Risk Betas}

Weak-instrument-robust inference:

Underidentification test

Weak identification test

\section{Panel B: Exchange Rate Risk Beta}

Weak-instrument-robust inference:

Underidentification test

Weak identification test

\section{Panel C: Credit Risk Beta}

Weak-instrument-robust inference:

Underidentification test

Weak identification test

Anderson-Rubin Wald test ( $p$-value)

Kleibergen-Paap rk LM statistic ( $p$-value)

Cragg-Donald Wald F statistic

Kleibergen-Paap Wald rk F statistic

Anderson-Rubin Wald test ( $p$-value)

Kleibergen-Paap rk LM statistic ( $p$-value)

Cragg-Donald Wald F statistic

Kleibergen-Paap Wald rk F statistic

Anderson-Rubin Wald test ( $p$-value)

Kleibergen-Paap rk LM statistic ( $p$-value)

Cragg-Donald Wald F statistic

Kleibergen-Paap Wald rk F statistic

$$
\begin{aligned}
& 23.45^{\text {*** }}(0.000) \\
& 40.26^{* * * *}(0.000) \\
& 2942.68^{* * *} \\
& 34.48^{* * * *} \\
& 39.51^{\text {****}}(0.000) \\
& 82.61^{\text {****}}(0.000) \\
& 1684.93^{\text {*** }} \\
& 36.845^{\text {*** }} \\
& 19.45^{\text {*** }}(0.000) \\
& 33.20^{* * *}(0.000) \\
& 1.4 \mathrm{e}+04^{* * *} \\
& 150.687^{* * *}
\end{aligned}
$$

$$
12.46^{* * *}(0.0060)
$$$$
42.130^{* * *}(0.000)
$$$$
457.639^{* * * *}
$$$$
33.96^{* * *}
$$$$
32.50^{* * *}(0.000)
$$$$
72.71^{* * *}(0.000)
$$$$
219.89^{* * *}
$$

$37.65^{* * *}$

$32.71^{\text {**** }}(0.000)$

$35.88^{* * * *}(0.000)$

$3938.86^{* * *}$

$218.270^{* * * *}$
$13.16^{* *}(0.011)$

$31.29^{* * *}(0.000)$

$1751.21^{* * * *}$

$41.66^{* * *}$

$14.97^{* * * *}(0.002)$

$13.65^{* * *}(0.003)$

$1605.38^{* * *}$

$16.45^{* * *}$

$12.64^{* *}(0.012)$

$20.21^{* * *}(0.001)$

$2119.16^{* * *}$

$44.36^{* * *}$

Note. Superscripts $* * *, * *, *$ indicate significance levels of $1 \%, 5 \%$, and $10 \%$ respectively. 
Table A.2. Determinants of Interest Rate, Exchange Rate, and Credit Risk Betas

\begin{tabular}{|c|c|c|c|c|c|c|c|c|c|}
\hline Variable & & tal BHCs & & & Large BHC & & & Small BHCs & \\
\hline Panel A: Interest Rate Risl & eign Expos & Instrumer & Variable) & & & & & & \\
\hline Interest Margin & $\begin{array}{c}4.542 * * * \\
(6.26)\end{array}$ & $\begin{array}{l}0.784 \\
(0.44)\end{array}$ & $\begin{array}{c}-8.467 * * * \\
(-2.73)\end{array}$ & $\begin{array}{c}8.028 * * \\
(1.99)\end{array}$ & $\begin{array}{l}8.515^{*} \\
(1.95)\end{array}$ & $\begin{array}{c}218.4 * * \\
(2.53)\end{array}$ & $\begin{array}{c}4.193 * * * \\
(5.85)\end{array}$ & $\begin{array}{c}-0.0626 \\
(-0.03)\end{array}$ & $\begin{array}{l}-1.795 \\
(-1.56)\end{array}$ \\
\hline C\&I Loans & $\begin{array}{l}-0.385 \\
(-0.99)\end{array}$ & $\begin{array}{c}2.220 * * \\
(2.26)\end{array}$ & $\begin{array}{c}8.888 * * * \\
(3.60)\end{array}$ & $\begin{array}{c}12.20^{* * * *} \\
(4.11)\end{array}$ & $\begin{array}{c}14.09 * * * \\
(4.35)\end{array}$ & $\begin{array}{l}0.138 \\
(0.14)\end{array}$ & $\begin{array}{c}-0.800 * * \\
(-2.09)\end{array}$ & $\begin{array}{l}0.699 \\
(0.73)\end{array}$ & $\begin{array}{l}0.593 \\
(0.67)\end{array}$ \\
\hline Mortgage Loans & $\begin{array}{l}0.129 \\
(0.54)\end{array}$ & $\begin{array}{l}0.754 \\
(1.20)\end{array}$ & $\begin{array}{c}5.309 * * * \\
(3.52)\end{array}$ & $\begin{array}{c}9.000 * * * \\
(3.92)\end{array}$ & $\begin{array}{c}9.662 * * * \\
(4.07)\end{array}$ & $\begin{array}{l}0.335 \\
(0.51)\end{array}$ & $\begin{array}{c}0.0504 \\
(0.22)\end{array}$ & $\begin{array}{l}0.195 \\
(0.32)\end{array}$ & $\begin{array}{l}0.148 \\
(0.29)\end{array}$ \\
\hline Other Loans & $\begin{array}{c}-1.552 * * * \\
(-3.22)\end{array}$ & $\begin{array}{l}-1.346 \\
(-1.09)\end{array}$ & $\begin{array}{c}14.05^{* * * *} \\
(3.56)\end{array}$ & $\begin{array}{c}5.139 * * \\
(2.05)\end{array}$ & $\begin{array}{c}5.608 * * \\
(2.03)\end{array}$ & $\begin{array}{l}0.856 \\
(1.24)\end{array}$ & $\begin{array}{c}-1.657 * * * \\
(-3.70)\end{array}$ & $\begin{array}{c}-3.811 * * * \\
(-3.18)\end{array}$ & $\begin{array}{l}1.094 * \\
(1.94)\end{array}$ \\
\hline Domestic Deposits & $\begin{array}{c}-0.861 * * * \\
(-3.34)\end{array}$ & $\begin{array}{c}1.787 * * * \\
(3.08)\end{array}$ & $\begin{array}{c}1.978 * * * \\
(2.75)\end{array}$ & $\begin{array}{l}1.615 \\
(0.95)\end{array}$ & $\begin{array}{l}1.011 \\
(0.57)\end{array}$ & $\begin{array}{l}-0.249 \\
(-0.49)\end{array}$ & $\begin{array}{c}-1.580 * * * \\
(-6.41)\end{array}$ & $\begin{array}{l}0.381 \\
(0.66)\end{array}$ & $\begin{array}{c}0.0531 \\
(0.17)\end{array}$ \\
\hline GAP Ratio & $\begin{array}{c}-0.00693 \\
(-1.57)\end{array}$ & $\begin{array}{c}-0.00453 \\
(-0.92)\end{array}$ & $\begin{array}{c}-0.0245 * * * \\
(-5.49)\end{array}$ & $\begin{array}{c}0.00158 \\
(0.29)\end{array}$ & $\begin{array}{c}0.00516 \\
(0.88)\end{array}$ & $\begin{array}{c}-0.00199 * \\
(-1.80)\end{array}$ & $\begin{array}{l}-0.0274 \\
(-1.16)\end{array}$ & $\begin{array}{c}-0.106 * * \\
(-2.14)\end{array}$ & $\begin{array}{c}-0.0868 * * \\
(-2.37)\end{array}$ \\
\hline Size & $\begin{array}{c}0.641 * * * \\
(10.41)\end{array}$ & $\begin{array}{c}0.917 * * * \\
(5.78)\end{array}$ & $\begin{array}{c}4.323 * * * \\
(3.95)\end{array}$ & $\begin{array}{c}1.048 * * \\
(2.58)\end{array}$ & $\begin{array}{c}1.163 * * * \\
(2.81)\end{array}$ & $\begin{array}{c}0.0123 \\
(0.19)\end{array}$ & $\begin{array}{c}0.474 * * * \\
(8.65)\end{array}$ & $\begin{array}{c}0.426 * * * \\
(3.05)\end{array}$ & $\begin{array}{l}0.160 \\
(1.26)\end{array}$ \\
\hline Capital Ratio & $\begin{array}{c}0.0952 \\
(0.86)\end{array}$ & $\begin{array}{c}2.943 * * * \\
(3.57)\end{array}$ & $\begin{array}{c}6.951 * * * \\
(2.92)\end{array}$ & $\begin{array}{l}4.496 \\
(1.08)\end{array}$ & $\begin{array}{l}3.665 \\
(0.74)\end{array}$ & $\begin{array}{l}-2.885 \\
(-1.45)\end{array}$ & $\begin{array}{r}0.0417 \\
(0.69)\end{array}$ & $\begin{array}{l}1.209 \\
(1.46)\end{array}$ & $\begin{array}{l}0.138 \\
(0.24)\end{array}$ \\
\hline GDP Growth & $\begin{array}{c}-0.00625 \\
(-1.28)\end{array}$ & $\begin{array}{c}-0.000173 \\
(-0.01)\end{array}$ & $\begin{array}{c}0.00539 \\
(0.51)\end{array}$ & $\begin{array}{l}0.0261 \\
(1.12)\end{array}$ & $\begin{array}{c}0.0283 \\
(1.17)\end{array}$ & $\begin{array}{c}0.00529 \\
(0.57)\end{array}$ & $\begin{array}{c}-0.0117 * * \\
(-2.36)\end{array}$ & $\begin{array}{c}-0.0399 * * * \\
(-2.97)\end{array}$ & $\begin{array}{c}0.00146 \\
(0.14)\end{array}$ \\
\hline Interest Rate Derivatives & $\begin{array}{c}0.116 * * * \\
(4.50)\end{array}$ & $\begin{array}{c}0.146 * * * \\
(4.60)\end{array}$ & $\begin{array}{l}1.127 * \\
(1.88)\end{array}$ & $\begin{array}{c}0.0962 * * * \\
\quad(3.29)\end{array}$ & $\begin{array}{c}0.106^{* * *} \\
(3.10)\end{array}$ & $\begin{array}{c}0.0126 * \\
(1.96)\end{array}$ & $\begin{array}{c}0.157 * * * \\
(2.99)\end{array}$ & $\begin{array}{c}0.200 * * * \\
(2.93)\end{array}$ & $\begin{array}{l}0.155 \\
(1.64)\end{array}$ \\
\hline L.Interest Rate Risk Beta & & & $\begin{array}{l}0.0361 \\
(1.04) \\
\end{array}$ & & & $\begin{array}{c}1.080 * * * \\
(81.24)\end{array}$ & & & $\begin{array}{l}0.256^{*} \\
(1.80) \\
\end{array}$ \\
\hline$N$ & 11795 & 4367 & 3866 & 754 & 719 & 725 & 11041 & 3648 & 3169 \\
\hline Adjusted-R2 & 0.199 & 0.138 & & 0.473 & 0.474 & & 0.191 & 0.0667 & \\
\hline $\mathrm{AR}(1)$ & & & 0.008 & & & 0.000 & & & 0.014 \\
\hline $\operatorname{AR}(2)$ & & & 0.676 & & & 0.206 & & & 0.436 \\
\hline Hansen J Statistic ( $p$-value) & & & $3.27(0.514)$ & & & $2.67(0.103)$ & & & $4.04(0.401)$ \\
\hline
\end{tabular}

Panel B: Exchange Rate Risk Beta (Interest Rate Exposure as Instrument Variable) 
Table A.2. Determinants of Interest Rate, Exchange Rate, and Credit Risk Betas

\section{Variable}

Assets in Foreign Currencies

Foreign Exchange Deposits

Size

Capital Ratio

GDP Growth

Exchange Rate Derivatives

L. Exchange Rate Risk Beta

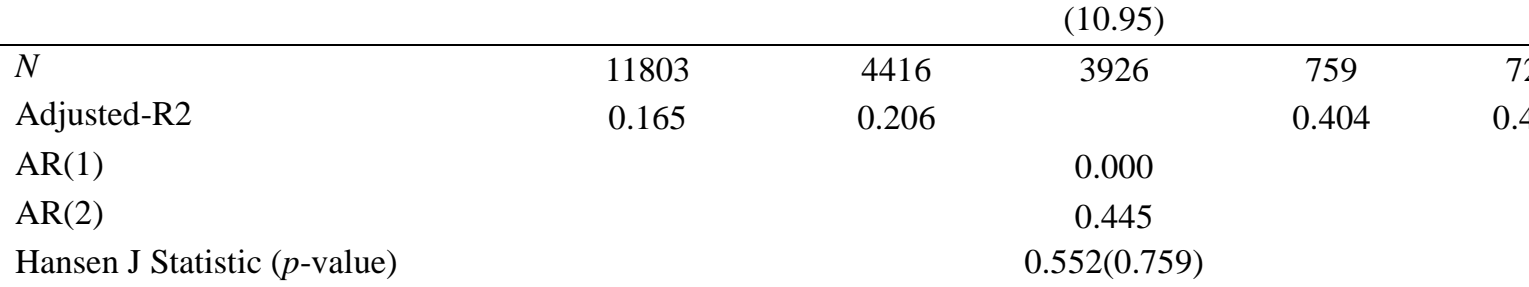

Market Liquidity

Funding Liquidity

Non-Performing Loans

Loan Charge-Offs

Loan Loss Provisions

$\begin{array}{ccc}-0.511 * * * & -0.416 * * * & -0.0891 \\ (-9.38) & (-5.21) & (-1.24) \\ -0.388 * * * & -0.636 * * * & 0.0139 \\ (-2.94) & (-4.04) & (0.14) \\ -4.717 * * * & -2.996 * * * & -0.345 \\ (-11.39) & (-6.01) & (-1.03) \\ -5.922 * * * & -4.308 * & -0.244 \\ (-2.68) & (-1.83) & (-0.24) \\ 6.488 * * * & 4.775 * * & 0.328\end{array}$

(3.19)
(2.08)
$-0.253$

(-1.11)

$-1.562 * * *$

$(-3.59)$

$-11.42 * * *$

(-7.45)

$-17.73 * * *$

$(-3.13)$

$12.56 * * *$

(0.30)
Large BHCs

0.778

(-3.19) (0.99)

$-2.116^{*}$

(-1.80)

0.114

(0.83)

$-2.805$

(-1.18)

0.0174

(0.92)

0.837 ***

(6.07)

0.158

(0.11)

0.181

(0.41)

0.283

(0.09)

0.0172

(0.83)

0.145

(1.54)

$1.686 * * *$

(3.80)

728
0.408

707

0.003

0.110

2.52(0.640)

\begin{tabular}{|c|c|c|}
\hline \multicolumn{3}{|c|}{ Small BHCs } \\
\hline $4.864 * * *$ & $3.875 * * *$ & -0.558 \\
\hline$(4.88)$ & $(3.15)$ & $(-1.06)$ \\
\hline $1.450 * *$ & -1.498 & 0.220 \\
\hline (1.98) & $(-1.22)$ & $(0.24)$ \\
\hline$-0.118 * *$ & $-0.212^{* *}$ & $0.220 *$ \\
\hline$(-2.23)$ & $(-2.36)$ & (1.73) \\
\hline-0.0499 & -0.163 & 0.290 \\
\hline$(-0.87)$ & $(-0.45)$ & (1.04) \\
\hline $0.0169 * * *$ & $0.0232 * * *$ & -0.0107 \\
\hline (3.04) & (2.84) & $(-1.39)$ \\
\hline $0.791 * * *$ & $0.920 * * *$ & $0.130 * * *$ \\
\hline \multirow[t]{3}{*}{$(5.29)$} & (4.19) & $(4.40)$ \\
\hline & & $0.981 * * *$ \\
\hline & & $(10.61)$ \\
\hline 11044 & 3688 & 3219 \\
\hline \multirow[t]{4}{*}{0.160} & 0.193 & \\
\hline & & 0.000 \\
\hline & & 0.816 \\
\hline & & $1.05(0.789)$ \\
\hline
\end{tabular}

$\begin{array}{lll}(2.70) & (3.92) \quad(-0.25)\end{array}$


Table A.2. Determinants of Interest Rate, Exchange Rate, and Credit Risk Betas

\begin{tabular}{|c|c|c|c|c|c|c|c|c|c|}
\hline Variable & \multicolumn{3}{|c|}{ Total BHCs } & \multicolumn{3}{|c|}{ Large BHCs } & \multicolumn{3}{|c|}{ Small BHCs } \\
\hline Size & $\begin{array}{c}0.0496 * * * \\
(2.83)\end{array}$ & $\begin{array}{l}-0.0180 \\
(-0.68)\end{array}$ & $\begin{array}{c}-0.0206 \\
(-0.69)\end{array}$ & $\begin{array}{c}0.207 * * * \\
(4.14)\end{array}$ & $\begin{array}{c}0.278 * * * \\
(6.01)\end{array}$ & $\begin{array}{c}0.00968 \\
(0.02)\end{array}$ & $\begin{array}{c}0.0658 * * * \\
(3.60)\end{array}$ & $\begin{array}{c}0.00813 \\
(0.28)\end{array}$ & $\begin{array}{c}-0.00276 \\
(-0.07)\end{array}$ \\
\hline Capital Ratio & $\begin{array}{c}-0.00646 \\
(-0.69)\end{array}$ & $\begin{array}{l}-0.0400 \\
(-0.46)\end{array}$ & $\begin{array}{c}0.117 * * \\
(1.97)\end{array}$ & $\begin{array}{c}2.162 * * * \\
(3.03)\end{array}$ & $\begin{array}{c}1.728 * * \\
(2.17)\end{array}$ & $\begin{array}{l}-0.367 \\
(-0.09)\end{array}$ & $\begin{array}{c}-0.00130 \\
(-0.11)\end{array}$ & $\begin{array}{c}0.0462 \\
(0.53)\end{array}$ & $\begin{array}{l}0.117 \\
(1.39)\end{array}$ \\
\hline GDP Growth & $\begin{array}{c}0.00617 * * * \\
(3.40)\end{array}$ & $\begin{array}{c}0.00622 * * \\
(2.27)\end{array}$ & $\begin{array}{c}0.00583^{* *} \\
(2.09)\end{array}$ & $\begin{array}{c}0.0161 * * \\
(2.41)\end{array}$ & $\begin{array}{c}0.0164 * * \\
(2.54)\end{array}$ & $\begin{array}{c}-0.0187 \\
(-1.14)\end{array}$ & $\begin{array}{c}0.00520 * * * \\
(2.80)\end{array}$ & $\begin{array}{c}0.00454 \\
(1.58)\end{array}$ & $\begin{array}{c}0.00836^{* *} \\
(2.02)\end{array}$ \\
\hline Credit Derivatives & $\begin{array}{c}0.0761 * * * \\
(4.73)\end{array}$ & $\begin{array}{c}0.0746 * * * \\
(4.37)\end{array}$ & $\begin{array}{c}0.0257 * * \\
(2.23)\end{array}$ & $\begin{array}{c}0.103 * * * \\
(5.88)\end{array}$ & $\begin{array}{c}0.0948 * * * \\
(5.71)\end{array}$ & $\begin{array}{l}0.0111 \\
(0.11)\end{array}$ & $\begin{array}{c}0.0708 * \\
(1.76)\end{array}$ & $\begin{array}{l}0.102 \\
(1.40)\end{array}$ & $\begin{array}{c}0.0426 * * * \\
(2.75)\end{array}$ \\
\hline L. Credit Risk Beta & & & $\begin{array}{c}-0.654 * * * \\
(-6.96) \\
\end{array}$ & & & $\begin{array}{l}0.332 \\
(1.24) \\
\end{array}$ & & & $\begin{array}{c}0.906 * * * \\
(7.87)\end{array}$ \\
\hline$N$ & 10984 & 4152 & 3705 & 697 & 667 & 646 & 10287 & 3485 & 3059 \\
\hline Adjusted-R2 & 0.163 & 0.216 & & 0.594 & 0.620 & & 0.149 & 0.168 & \\
\hline $\operatorname{AR}(1)$ & & & 0.000 & & & 0.02 & & & 0.000 \\
\hline $\operatorname{AR}(2)$ & & & 0.789 & & & 0.267 & & & 0.408 \\
\hline Hansen J Statistic ( $p$-value) & & & $2.02(0.364)$ & & & $10.18(0.179)$ & & & $0.15(0.928)$ \\
\hline Estimation Method & FE & IV & GMM & FE & IV & GMM & FE & IV & GMM \\
\hline
\end{tabular}




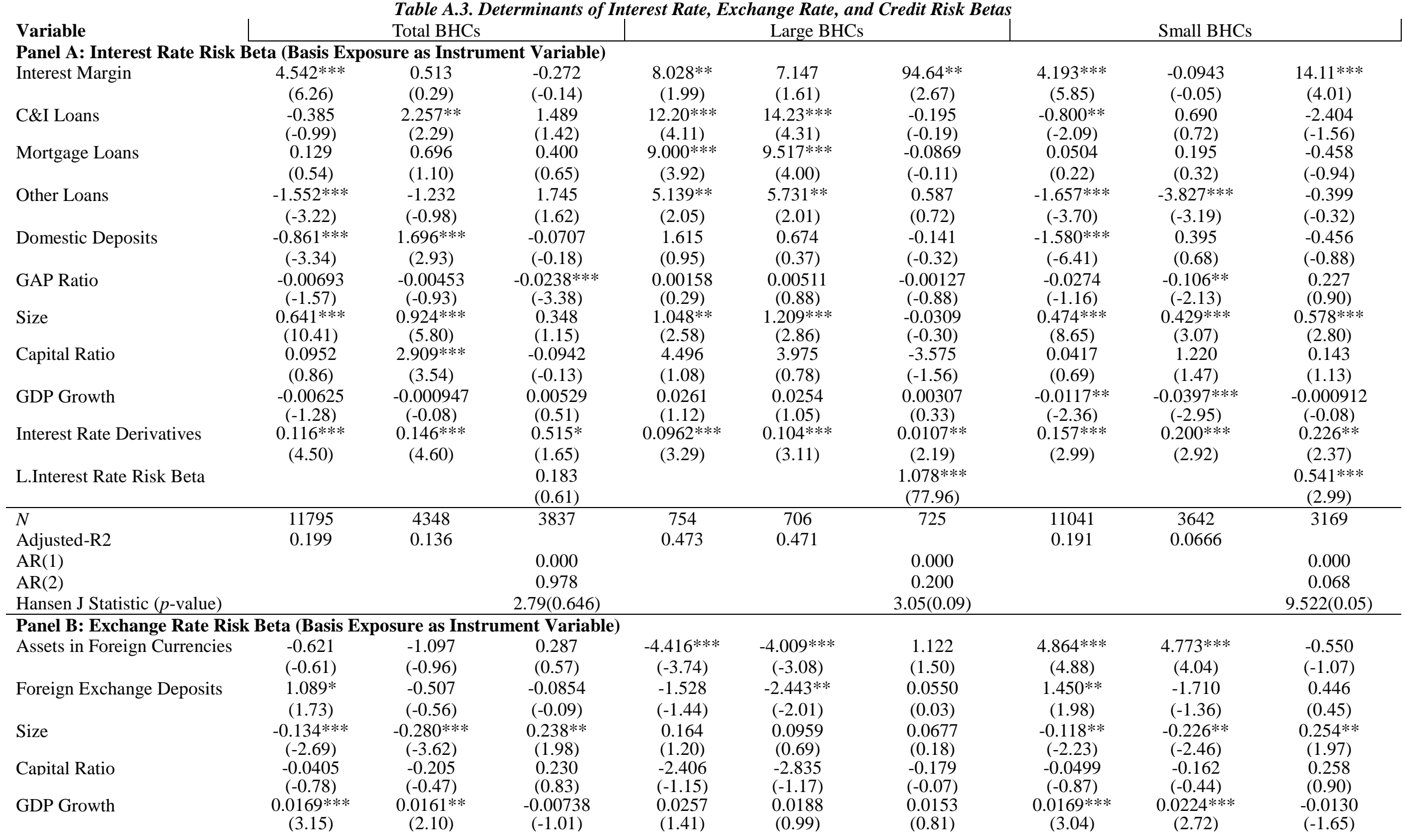


Table A.3. Determinants of Interest Rate, Exchange Rate, and Credit Risk Betas

Variable

Exchange Rate Derivatives

Total BHCs

L. Exchange Rate Risk Beta

$\begin{array}{cc} & \text { Total BHCs } \\ 0.545^{* * *} & 0.678^{* * *} \\ (6.31) & (5.81)\end{array}$

$\begin{array}{ccc} & & \text { Large BHCs } \\ 0.153 * * * & 0.721 * * * & 0.857 * * * \\ (4.97) & (6.84) & (5.95) \\ 0.993 * * * & & \end{array}$

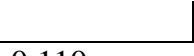

$N$
Adjusted-R2

$0.993 * * *$

(6.84) $\quad(5.95)$

0.110
$(1.35)$

$1.508 * * *$

$3841 \quad 759$

0.404

(4.49)

\begin{tabular}{ll} 
& Small BHCs \\
\hline $0.791^{* * *}$ & $0.934 * * *$
\end{tabular}

$0.934 * * *$
$(4.15)$

(5.29)

(4.15)

$0.135^{* * *}$

(4.62)

$\operatorname{AR}(1)$

$\operatorname{AR}(2)$

0.165

0.203

0.000

0.472

711
0.404

0.002

0.128
$94(0.568)$

3643

(10.63)

Hansen J Statistic ( $p$-value)

Variable)

Market Liquidity

Funding Liquidity

Non-Performing Loans

Loan Charge-Offs

Loan Loss Provisions

Size

Capital Ratio

GDP Growth

Credit Derivatives

L. Credit Risk Beta

\begin{tabular}{cc}
\multicolumn{2}{c}{ Exposure as Instrument } \\
$-0.511 * * *$ & $-0.440^{* * *}$ \\
$(-9.38)$ & $(-5.48)$ \\
$-0.388^{* * *}$ & $-0.663 * * *$ \\
$(-2.94)$ & $(-4.04)$ \\
$-4.717 * * *$ & $-3.147 * * *$ \\
$(-11.39)$ & $(-6.25)$ \\
$-5.922 * * *$ & $-4.501 *$ \\
$(-2.68)$ & $(-1.94)$ \\
$6.488^{* * *}$ & $4.960 * *$ \\
$(3.19)$ & $(2.20)$ \\
$0.0496 * * *$ & -0.00257 \\
$(2.83)$ & $(-0.10)$ \\
-0.00646 & -0.0133 \\
$(-0.69)$ & $(-0.15)$ \\
$0.00617 * * *$ & $0.00609 * *$ \\
$(3.40)$ & $(2.22)$ \\
$0.0761 * * *$ & $0.0734 * * *$ \\
$(4.73)$ & $(4.33)$
\end{tabular}

$-0.111$

$(-1.49)$

0.0233

$(0.23)$

$-0.532$

$(-1.62)$

$-0.200$

$(-0.20)$

0.193

$(0.18)$
-0.0140

$(-0.47)$
$0.137 * *$

$0.137 * *$
$(2.19)$

$(2.19)$
$.00570 * *$

(2.04)

$0.0243 * * *$

$(2.62)$
$0.703 * * *$

$-0.253 \quad-0.439 *$

$-0.262$

$(-1.11) \quad(-1.95)$

$-1.562 * * *$

$-1.601 * * *$

$(-0.05)$

$(-3.59)$

$(-3.76)$

$13.62 * *$

$-11.42 * * * \quad-12.54 * * *$

(2.14)

$(-7.45)$

$(-8.51)$

$-39.84$

$-17.73 * * *$

$-23.53 * * *$

(0.77)

(-3.13)

$(-5.16)$

$12.56 * * *$

$0.207 * * *$

$16.65 * * *$

$-41.29$

(-1.19)

$(3.91)$
$0.277 * * *$

80.36**

(4.14)

(5.99)

-0.390
$(-0.72)$

$2.162 * * *$

$(5.99)$
$1.720 * *$

$(-0.72)$
$-33.11 *$

$0.0161 * *$

(2.16)

$(-1.81)$

$0.0164 * *$

$$
\text { (2.54) }
$$

$0.103 * * *$

$0.0961 * * *$

$-0.0677$

(-1.63)

(5.77)

$0.998 * *$

$(3.75)$
$(0.0658$

0.0658 $\frac{(-7.37)}{3664}$

(5.88)

646

11044

0.160

0.193

0.000

0.847

$1.29(0.731)$

Adjusted-R2

10984

4121

3664

697

697
0.594

667

646

$-0.518 * * *$

$-0.462 * * *$

$-0.346 * * \quad(-5.54)$

$-0.547 * * * \quad-0.0500$

$(-3.04) \quad(-0.40)$

$\begin{array}{ccc}(-2.48) & (-3.04) & (-0.40) \\ -4.269 * * * & -1.570 * * * & 0.266\end{array}$

$\operatorname{AR}(1)$

0.000

0.001

0.482

$(-10.17) \quad(-3.22)$

(0.86)

$\begin{array}{lll}-3.878 * & -0.743 & 0.165\end{array}$

$\begin{array}{lll}(-1.65) & (-0.32) & (0.14)\end{array}$

$5.592 * * * \quad 2.570 \quad 2.685 * *$

$\begin{array}{lll}(2.60) & (1.14) & (2.23) \\ 0.0658 * * * & 0.0264 & 0.00147\end{array}$

$0.0658 * * * \quad 0.0264 \quad 0.00147$

$\begin{array}{lll}(3.60) & (0.92) & (0.03)\end{array}$

$\begin{array}{lll}-0.00130 & 0.0735 & 0.0867\end{array}$

AR(2)

Hansen J Statistic ( $p$-value)

$0.460(0.795)$

Estimation Method

IV GMM FE

FE IV

6.91(0.938)

$(-0.11)$

0.0735
$(0.86)$

0.0867
$(1.16)$

$0.00520^{* * *} \quad 0.00437 \quad 0.00701^{*}$

$\begin{array}{lll}(2.80) & (1.52) & (1.73)\end{array}$

$0.0708 *$

(1.76)

(1.52)

$(1.73)$

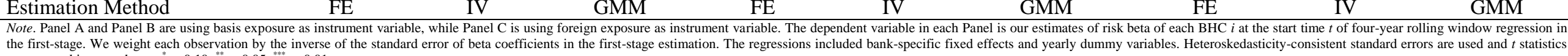

are reported in parentheses. " $p<0.10, " p<0.05,{ }^{3 * *} p<0.01$

SOure: Financial Statements data from FR Y-9C; Risk betas are computed from the four-factor model using data from Center for Research in Security Prices (CRSP) database and Federal Reserve monthly Statistical Releases. 
Table A.4. Determinants of Interest Rate, Exchange Rate, and Credit Risk Betas

\begin{tabular}{|c|c|c|c|c|c|c|c|c|c|}
\hline \multirow{2}{*}{$\begin{array}{l}\text { Variable } \\
\text { Panel A: Intere }\end{array}$} & \multicolumn{3}{|c|}{ Total BHCs } & \multicolumn{3}{|c|}{ Large BHCs } & \multicolumn{3}{|c|}{ Small BHCs } \\
\hline & ta (Foreign $\mathrm{E}$ & osure and $\mathrm{Bz}$ & s Exposure as & trument $V$ & ibles) & & & & \\
\hline \multirow{2}{*}{ Interest Margin } & $3.238 * * *$ & 0.878 & -1.263 & 7.394 & 6.849 & 21.91 & $2.823 * * *$ & 0.193 & 4.625 \\
\hline & $(3.49)$ & $(0.42)$ & $(-0.65)$ & $(1.52)$ & $(1.28)$ & $(1.03)$ & $(3.08)$ & $(0.10)$ & $(0.26)$ \\
\hline \multirow[t]{2}{*}{ C\&I Loans } & $-1.601 * * *$ & 0.168 & 0.774 & $16.65 * * *$ & $18.01 * * *$ & -0.616 & $-2.228 * * *$ & -1.727 & -2.415 \\
\hline & $(-3.38)$ & $(0.15)$ & $(0.75)$ & (4.82) & $(4.65)$ & $(-0.64)$ & $(-4.76)$ & $(-1.63)$ & $(-1.15)$ \\
\hline \multirow[t]{2}{*}{ Mortgage Loans } & 0.191 & 0.653 & 0.913 & $11.64 * * *$ & $11.86 * * *$ & -0.655 & 0.125 & 0.287 & -0.412 \\
\hline & $(0.71)$ & (1.05) & (1.24) & $(4.44)$ & $(4.33)$ & $(-0.66)$ & $(0.47)$ & $(0.48)$ & $(-0.50)$ \\
\hline \multirow[t]{2}{*}{ Other Loans } & -0.939 & 0.187 & -0.402 & 2.073 & 2.901 & -0.807 & -0.618 & -0.786 & 0.372 \\
\hline & $(-1.58)$ & $(0.12)$ & $(-0.28)$ & $(0.70)$ & $(0.86)$ & $(-0.67)$ & $(-1.13)$ & $(-0.64)$ & $(0.21)$ \\
\hline \multirow[t]{2}{*}{ Domestic Deposits } & -0.446 & $1.792 * * *$ & -0.00951 & 1.771 & 1.207 & -0.000953 & $-1.280 * * *$ & 0.496 & -0.153 \\
\hline & $(-1.45)$ & $(2.90)$ & $(-0.02)$ & $(0.89)$ & $(0.57)$ & $(-0.00)$ & $(-4.30)$ & $(0.86)$ & $(-0.16)$ \\
\hline \multirow[t]{2}{*}{ GAP Ratio } & $-0.00918^{* *}$ & $-0.00873^{* *}$ & $-0.0310^{* * *}$ & 0.00964 & $0.0175 * *$ & -0.000508 & -0.000455 & 0.0790 & 0.262 \\
\hline & $(-2.52)$ & $(-2.07)$ & $(-6.37)$ & $(1.50)$ & $(2.35)$ & $(-0.26)$ & $(-0.04)$ & $(1.57)$ & $(1.40)$ \\
\hline \multirow[t]{2}{*}{ Size } & $0.482 * * *$ & $0.713 * * *$ & -0.00317 & $1.021 * *$ & $1.119 * *$ & -0.233 & $0.305^{* * *}$ & $0.272 *$ & 0.214 \\
\hline & $(6.52)$ & (4.39) & $(-0.01)$ & $(2.19)$ & $(2.30)$ & $(-1.01)$ & $(4.45)$ & (1.90) & (1.14) \\
\hline \multirow[t]{2}{*}{ Capital Ratio } & -0.0251 & 0.180 & -0.00524 & $13.21 * * *$ & $12.77 * *$ & -3.123 & -0.0916 & 0.0224 & -0.0952 \\
\hline & $(-0.24)$ & $(1.14)$ & $(-0.57)$ & $(2.59)$ & $(2.09)$ & $(-1.25)$ & $(-1.31)$ & $(0.53)$ & $(-0.67)$ \\
\hline \multirow[t]{2}{*}{ GDP Growth } & $-0.0177 * * *$ & $-0.0286^{* *}$ & -0.00268 & 0.00875 & 0.000383 & 0.00286 & $-0.0254 * * *$ & $-0.0679 * * *$ & 0.00332 \\
\hline & $(-2.80)$ & $(-2.08)$ & $(-0.22)$ & $(0.29)$ & $(0.01)$ & $(0.29)$ & $(-3.96)$ & $(-4.68)$ & $(0.19)$ \\
\hline \multirow[t]{2}{*}{ Interest Rate Derivatives } & $0.117 * * *$ & $0.145^{* * *}$ & 0.233 & $0.0910 * *$ & $0.0825^{*}$ & $0.0143 * *$ & 0.0792 & 0.0825 & 0.129 \\
\hline & $(3.71)$ & $(3.65)$ & $(0.95)$ & $(2.37)$ & $(1.79)$ & $(2.73)$ & (1.43) & (1.19) & $(1.25)$ \\
\hline \multirow[t]{2}{*}{ L.Interest Rate Risk Beta } & & & $0.542 * *$ & & & $1.067 * * *$ & & & $0.711 * * *$ \\
\hline & & & $(2.27)$ & & & $(108.02)$ & & & $(6.52)$ \\
\hline$N$ & 14128 & 6154 & 5476 & 792 & 744 & 762 & 13336 & 5410 & 4714 \\
\hline Adjusted-R2 & 0.106 & 0.0633 & & 0.460 & 0.461 & & 0.0985 & 0.0209 & \\
\hline $\operatorname{AR}(1)$ & & & 0.014 & & & 0.000 & & & 0.000 \\
\hline $\operatorname{AR}(2)$ & & & 0.736 & & & 0.237 & & & 0.487 \\
\hline Hansen J Statistic ( $p$-value) & & & $11.74(0.228)$ & & & $0.0200(0.990)$ & & & $2.402(0.493)$ \\
\hline
\end{tabular}

Panel B: Exchange Rate Risk Beta (Interest Rate Exposure and Basis Exposure as Instrument Variables) 
Table A.4. Determinants of Interest Rate, Exchange Rate, and Credit Risk Betas

\section{Variable}

Assets in Foreign Currencies

Foreign Exchange Deposits

Size

\begin{tabular}{|ll} 
& Large BHCs \\
\hline$-5.007 * * *$ & $-4.760 * * *$
\end{tabular}

$(-4.14) \quad(-3.60)$

0.0423

$(0.04)$

0.150

$-0.504$

$(-0.42)$

0.0671

(1.16)

$-3.260 *$

$(-1.67)$

(0.06)

0.00678

(1.38)

$0.600 * * *$

(6.66)

(1.89)

$0.696 * * *$

(5.74)

0.000230

(0.18)

$-0.00423$

$(-0.56)$

(4.31)

$-0.000443$

(0.53)

$-3.341$

(-1.52)

$(-0.50)$

$0.693 * * * \quad 0.794 * * *$

(5.44)

(6.36)
Capital Ratio

GDP Growth

Exchange Rate Derivatives

L. Exchange Rate Risk Beta

$N$

Adjusted-R2

$\mathrm{AR}(1)$

$\mathrm{AR}(2)$

Hansen J Statistic ( $p$-value)

0.558(0.906)

$0.149 * * *$

$1.123 * * *$

(13.03)

$\begin{array}{ll}14136 & 6155 \\ 0.189 & 0.240\end{array}$

0.240

5474

0.000

\begin{tabular}{l}
-0.00696 \\
\hline
\end{tabular}

Market Liquidity

Funding Liquidity

Non-Performing Loans

Loan Charge-Offs

Loan Loss Provisions

$\begin{array}{cc}-0.134 * * * & -0.128 * * * \\ (-4.98) & (-2.99) \\ -0.181 * * * & -0.216 * * \\ (-3.25) & (-2.41) \\ -1.871 * * * & -0.950 * * * \\ (-9.93) & (-4.75) \\ -1.710 & -2.783 * * \\ (-1.48) & (-2.34) \\ 3.857 * * * & 3.795 * * *\end{array}$

(3.91)

(3.54)
-0.00696
$(-0.23)$

$-0.000983$

$(-0.03)$

0.0622

(0.59)

$-0.353$

(-0.79)

0.377

|

0.785

(1.07)

0.371

(0.33)

$-0.0643$

$(-0.20)$

$-1.066$

$(-0.38)$

0.00457

(0.17)

0.0530

(1.23)

$1.218 * * *$

(5.48)

$\begin{array}{cc}797 & 749 \\ 0.441 & 0.463\end{array}$

(0.83)
Instrument Variables)

$\begin{array}{ccc}0.334 * * * & 0.315 * * * & 0.228 \\ (3.39) & (3.07) & (0.97) \\ -1.093 * * * & -1.115 * * * & 0.616^{*} \\ (-4.37) & (-4.53) & (2.05) \\ -6.241 * * * & -6.637 * * * & 9.034 * * * \\ (-7.14) & (-7.25) & (2.86) \\ -12.41 * * * & -16.16^{* * *} & -9.121^{* *} \\ (-3.21) & (-4.95) & (-2.18) \\ 8.545 * * & 10.24 * * * & 20.35 * * \\ (2.38) & (3.02) & (2.34)\end{array}$

8

\begin{tabular}{ccc|}
\multicolumn{3}{c}{ Small BHCs } \\
\hline $4.954 * * *$ & $3.896 * * *$ & $-1.555^{*}$ \\
$(5.02)$ & $(2.78)$ & $(-1.72)$ \\
0.595 & -0.789 & 1.308 \\
$(0.78)$ & $(-0.65)$ & $(0.98)$ \\
-0.0305 & -0.0910 & $0.282^{* *}$ \\
$(-0.64)$ & $(-1.12)$ & $(2.40)$ \\
-0.00229 & -0.00811 & 0.000709 \\
$(-0.16)$ & $(-0.78)$ & $(0.52)$ \\
$0.00886 *$ & $0.0224 * * *$ & -0.0115 \\
$(1.75)$ & $(3.17)$ & $(-1.33)$ \\
$0.826 * * *$ & $0.908 * * *$ & $0.101 * * *$ \\
$(4.82)$ & $(3.68)$ & $(3.08)$ \\
& & $1.237 * * *$ \\
& & $(5.80)$ \\
\hline 13339 & 5406 & 4757 \\
0.182 & 0.217 & \\
& & 0.000 \\
& & 0.033
\end{tabular}

16.33(0.003) 
Table A.4. Determinants of Interest Rate, Exchange Rate, and Credit Risk Betas

\section{Variable}

Size

Capital Ratio

GDP Growth

Credit Derivatives

L. Credit Risk Beta

Adjusted-R2

$\operatorname{AR}(1)$

$\operatorname{AR}(2)$

Hansen J Statistic ( $p$-value)

Estimation Method

FE

FE
Total BHCs

$-0.00759 \quad-0.0197$

$(-0.80)$

0.00266

$(-1.31)$

-0.00214
$(-0.59)$

0.000603

0.00214

$(0.72)$

(1.44)

0.0126

0.0177

(1.16)

(1.40)

\begin{tabular}{c|} 
\\
$-0.0349 * * *$ \\
$(-3.88)$
\end{tabular}

$(-3.88)$

0.000250

(0.60)

0.00183

(1.51)

0.00741

\begin{abstract}
(1.19)
\end{abstract}
$-0.354 * * *$

5934

13359

0.0772

$(-3.85)$

5307

0.000

0.148

2.932(0.402)

GMM

\begin{abstract}
IV
\end{abstract}
Large BHCs

$0.0967 * * * \quad 0.127 * * *$

(3.55)

$0.762 *$

(4.19)

0.462

$-0.00224$

(0.92)

$(-1.03)$

$-0.00339$

$(-1.46)$

$0.0579 * * *$

(5.36)

$0.0585^{* * *}$

(5.24)

737

0.564

707

0.585

685

0.041

0.246

2.355(0.502)

FE

GMM

IV

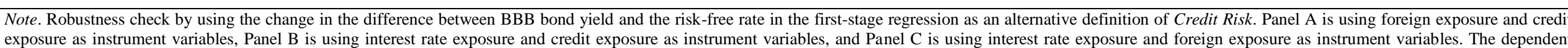

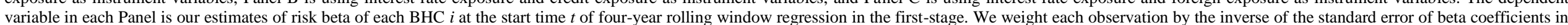

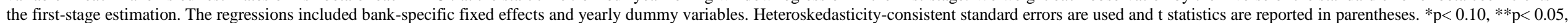
$* * * \mathrm{p}<0.01$.

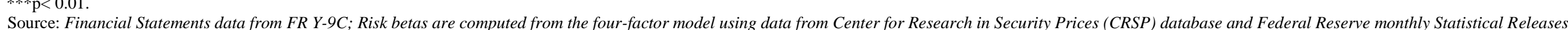


Table A.5. Determinants of Interest Rate, Exchange Rate, and Credit Risk Betas

\begin{tabular}{|c|c|c|c|c|c|c|c|c|c|}
\hline \multirow{3}{*}{$\begin{array}{l}\text { Variable } \\
\text { Panel A: Intere }\end{array}$} & \multicolumn{3}{|c|}{ Total BHCs } & \multicolumn{3}{|c|}{ Large BHCs } & \multicolumn{3}{|c|}{ Small BHCs } \\
\hline & ta (Foreign $F$ & osure as In & Iment Variab & & & & & & \\
\hline & $3.238 * * *$ & 1.019 & -1.282 & 7.394 & 8.170 & $-12.24 * * *$ & $2.823 * * *$ & 0.160 & $-2.729 *$ \\
\hline & $(3.49)$ & $(0.49)$ & $(-0.66)$ & (1.52) & $(1.56)$ & $(-3.59)$ & (3.08) & $(0.08)$ & $(-1.75)$ \\
\hline C\&I Loans & $-1.601 * * *$ & 0.218 & 0.859 & $16.65 * * *$ & $17.86 * * *$ & -0.901 & $-2.228 * * *$ & -1.617 & 1.030 \\
\hline & $(-3.38)$ & $(0.20)$ & $(0.84)$ & $(4.82)$ & $(4.71)$ & $(-0.94)$ & $(-4.76)$ & $(-1.51)$ & $(0.90)$ \\
\hline Mortgage Loans & 0.191 & 0.728 & 0.977 & $11.64 * * *$ & $11.97 * * *$ & 0.452 & 0.125 & 0.316 & 0.698 \\
\hline & $(0.71)$ & (1.17) & (1.34) & $(4.44)$ & $(4.40)$ & $(0.74)$ & $(0.47)$ & $(0.53)$ & $(1.31)$ \\
\hline Other Loans & -0.939 & 0.158 & -0.417 & 2.073 & 2.768 & 0.778 & -0.618 & -0.610 & 0.213 \\
\hline & $(-1.58)$ & $(0.11)$ & $(-0.29)$ & $(0.70)$ & $(0.85)$ & (1.10) & $(-1.13)$ & $(-0.49)$ & $(0.30)$ \\
\hline Domestic Deposits & -0.446 & $1.791 * * *$ & -0.0831 & 1.771 & 1.484 & 0.0585 & $-1.280 * * *$ & 0.419 & -0.0906 \\
\hline & $(-1.45)$ & $(2.89)$ & $(-0.19)$ & $(0.89)$ & $(0.71)$ & $(0.12)$ & $(-4.30)$ & $(0.73)$ & $(-0.20)$ \\
\hline GAP Ratio & $-0.00918 * *$ & $-0.00844 * *$ & $-0.0318 * * *$ & 0.00964 & $0.0177 * *$ & 0.00177 & -0.000455 & 0.0775 & $-0.0946 * *$ \\
\hline & $(-2.52)$ & $(-2.00)$ & $(-5.92)$ & $(1.50)$ & $(2.38)$ & $(1.23)$ & $(-0.04)$ & $(1.54)$ & $(-2.07)$ \\
\hline Size & $0.482 * * *$ & $0.723 * * *$ & -0.0214 & $1.021 * *$ & $1.076 * *$ & 0.129 & $0.305 * * *$ & $0.295 * *$ & 0.0980 \\
\hline & $(6.52)$ & $(4.42)$ & $(-0.05)$ & (2.19) & $(2.25)$ & $(1.36)$ & $(4.45)$ & $(2.02)$ & $(0.59)$ \\
\hline Capital Ratio & -0.0251 & 0.183 & -0.00434 & $13.21 * * *$ & $12.32 * *$ & -1.653 & -0.0916 & 0.0247 & -0.00254 \\
\hline & $(-0.24)$ & (1.14) & $(-0.48)$ & $(2.59)$ & $(2.07)$ & $(-0.77)$ & $(-1.31)$ & $(0.58)$ & $(-0.42)$ \\
\hline GDP Growth & $-0.0177 * * *$ & $-0.0271 * *$ & -0.00192 & 0.00875 & 0.00546 & 0.00816 & $-0.0254 * * *$ & $-0.0676 * * *$ & -0.00622 \\
\hline & $(-2.80)$ & $(-1.98)$ & $(-0.16)$ & $(0.29)$ & $(0.17)$ & $(0.73)$ & $(-3.96)$ & $(-4.66)$ & $(-0.42)$ \\
\hline Interest Rate Derivatives & $0.117 * * *$ & $0.144 * * *$ & 0.197 & $0.0910 * *$ & $0.0827 *$ & $0.0135 * *$ & 0.0792 & 0.0822 & 0.216 \\
\hline & $(3.71)$ & $(3.63)$ & $(1.01)$ & $(2.37)$ & $(1.78)$ & $(2.73)$ & $(1.43)$ & $(1.18)$ & $(0.51)$ \\
\hline L.Interest Rate Risk Beta & & & $0.594 * *$ & & & $1.073 * * *$ & & & $0.738 * *$ \\
\hline & & & $(2.30)$ & & & $(148.99)$ & & & $(2.03)$ \\
\hline$N$ & 14128 & 6173 & 5505 & 792 & 757 & 762 & 13336 & 5416 & 4711 \\
\hline Adjusted-R2 & 0.106 & 0.0645 & & 0.460 & 0.463 & & 0.0985 & 0.0211 & \\
\hline $\mathrm{AR}(1)$ & & & 0.013 & & & 0.000 & & & 0.035 \\
\hline $\operatorname{AR}(2)$ & & & 0.785 & & & 0.262 & & & 0.478 \\
\hline Hansen J Statistic ( $p$-value) & & & $10.57(0.227)$ & & & $0.0232(0.879)$ & & & $3.056(0.880)$ \\
\hline
\end{tabular}

Panel B: Exchange Rate Risk Beta (Interest Rate Exposure as Instrument Variable) 
Table A.5. Determinants of Interest Rate, Exchange Rate, and Credit Risk Betas

\begin{tabular}{|c|c|c|c|c|c|c|c|c|c|}
\hline \multirow{2}{*}{$\begin{array}{l}\text { Variable } \\
\text { Assets in Foreign Currencies }\end{array}$} & \multicolumn{3}{|c|}{ Total BHCs } & \multicolumn{3}{|c|}{ Large BHCs } & \multicolumn{3}{|c|}{ Small BHCs } \\
\hline & $\begin{array}{l}-0.570 \\
(-0.55)\end{array}$ & $\begin{array}{l}-1.806 \\
(-1.48)\end{array}$ & $\begin{array}{l}-0.316 \\
(-0.67)\end{array}$ & $\begin{array}{c}-5.007 * * * \\
(-4.14)\end{array}$ & $\begin{array}{c}-4.674 * * * \\
(-3.64)\end{array}$ & $\begin{array}{l}0.417 \\
(0.63)\end{array}$ & $\begin{array}{c}.954 * * * \\
(5.02)\end{array}$ & $\begin{array}{c}3.889 * * * \\
(2.79)\end{array}$ & $\begin{array}{c}1.677^{* *} \\
(2.54)\end{array}$ \\
\hline Foreign Exchange Deposits & $\begin{array}{l}0.820 \\
(1.30)\end{array}$ & $\begin{array}{l}0.367 \\
(0.42)\end{array}$ & $\begin{array}{l}0.341 \\
(0.49)\end{array}$ & $\begin{array}{c}0.0423 \\
(0.04)\end{array}$ & $\begin{array}{l}-0.376 \\
(-0.31)\end{array}$ & $\begin{array}{l}0.340 \\
(0.38)\end{array}$ & $\begin{array}{l}0.595 \\
(0.78)\end{array}$ & $\begin{array}{l}-0.793 \\
(-0.66)\end{array}$ & $\begin{array}{l}0.182 \\
(0.15)\end{array}$ \\
\hline Size & $\begin{array}{l}-0.0434 \\
(-0.96)\end{array}$ & $\begin{array}{l}-0.122^{*} \\
(-1.75)\end{array}$ & $\begin{array}{l}0.152 \\
(1.56)\end{array}$ & $\begin{array}{l}0.150 \\
(1.16)\end{array}$ & $\begin{array}{c}0.0843 \\
(0.67)\end{array}$ & $\begin{array}{l}-0.124 \\
(-0.33)\end{array}$ & $\begin{array}{l}-0.0305 \\
(-0.64)\end{array}$ & $\begin{array}{l}-0.0902 \\
(-1.13)\end{array}$ & $\begin{array}{c}-0.282^{* * * *} \\
(-2.64)\end{array}$ \\
\hline Capital Ratio & $\begin{array}{c}0.000939 \\
(0.06)\end{array}$ & $\begin{array}{c}-0.00971 \\
(-0.79)\end{array}$ & $\begin{array}{c}0.000339 \\
(0.27)\end{array}$ & $\begin{array}{l}-3.260^{*} \\
(-1.67)\end{array}$ & $\begin{array}{l}-3.262 \\
(-1.50)\end{array}$ & $\begin{array}{l}-1.675 \\
(-0.50)\end{array}$ & $\begin{array}{c}-0.00229 \\
(-0.16)\end{array}$ & $\begin{array}{c}-0.00700 \\
(-0.69)\end{array}$ & $\begin{array}{c}0.00334 \\
(1.20)\end{array}$ \\
\hline Exchange Rate Derivatives & $\begin{array}{c}0.600 * * * \\
(6.66)\end{array}$ & $\begin{array}{c}0.698^{* * * *} \\
(5.85)\end{array}$ & $\begin{array}{c}0.155^{* * *} \\
(4.17)\end{array}$ & $\begin{array}{c}0.693 * * * \\
(6.36)\end{array}$ & $\begin{array}{c}0.805 * * * \\
(5.61)\end{array}$ & $\begin{array}{l}0.0561 \\
(0.82)\end{array}$ & $\begin{array}{c}0.826 * * * \\
(4.82)\end{array}$ & $\begin{array}{c}0.901 * * * \\
(3.69)\end{array}$ & $\begin{array}{c}0.123 * * \\
(2.02)\end{array}$ \\
\hline L. Exchange Rate Risk Beta & & & $\begin{array}{c}1.098^{* * *} \\
(13.36)\end{array}$ & & & $\begin{array}{c}1.279^{* * * *} \\
(4.80)\end{array}$ & & & $\begin{array}{c}-0.661 * * * \\
(-5.04)\end{array}$ \\
\hline$N$ & 14136 & 6221 & 5565 & 797 & 766 & 742 & 13339 & 5455 & 4823 \\
\hline \multicolumn{10}{|c|}{ Panel C: Credit Risk Beta (Interest Rate Exposure as Instrument Variable) } \\
\hline Market Liquidity & $\begin{array}{c}-0.134 * * * \\
(-4.98)\end{array}$ & $\begin{array}{c}-0.117 * * * \\
(-2.73)\end{array}$ & $\begin{array}{c}-0.00539 \\
(-0.18)\end{array}$ & $\begin{array}{c}0.334 * * * \\
(3.39)\end{array}$ & $\begin{array}{c}0.315 * * * \\
(3.07)\end{array}$ & $\begin{array}{l}0.194 \\
(0.81)\end{array}$ & $\begin{array}{c}-0.139 * * * \\
(-5.07)\end{array}$ & $\begin{array}{c}-0.154 * * * \\
(-3.45)\end{array}$ & $\begin{array}{l}0.0665 \\
(1.65)\end{array}$ \\
\hline Funding Liquidity & $\begin{array}{c}-0.181 * * * \\
(-3.25)\end{array}$ & $\begin{array}{c}-0.187 * * \\
(-2.12)\end{array}$ & $\begin{array}{c}0.00368 \\
(0.11)\end{array}$ & $\begin{array}{c}-1.093 * * * \\
(-4.37)\end{array}$ & $\begin{array}{c}-1.115^{* * *} \\
(-4.53)\end{array}$ & $\begin{array}{c}0.676^{* *} \\
(2.25)\end{array}$ & $\begin{array}{c}-0.162 * * * \\
(-2.82)\end{array}$ & $\begin{array}{l}-0.0853 \\
(-0.88)\end{array}$ & $\begin{array}{c}-0.122 * * \\
(-2.53)\end{array}$ \\
\hline Non-Performing Loans & $\begin{array}{c}-1.871^{* * *} \\
(-9.93)\end{array}$ & $\begin{array}{c}-0.936 * * * \\
(-4.69)\end{array}$ & $\begin{array}{l}0.0786 \\
(0.75)\end{array}$ & $\begin{array}{c}-6.241 * * * \\
(-7.14)\end{array}$ & $\begin{array}{c}-6.637^{* * *} \\
(-7.25)\end{array}$ & $\begin{array}{c}8.002^{* *} \\
(2.38)\end{array}$ & $\begin{array}{c}-1.858 * * * \\
(-9.98)\end{array}$ & $\begin{array}{c}-0.751 * * * \\
(-4.16)\end{array}$ & $\begin{array}{c}0.250 * * \\
(2.17)\end{array}$ \\
\hline Loan Charge-Offs & $\begin{array}{l}-1.710 \\
(-1.48)\end{array}$ & $\begin{array}{c}-2.746 * * \\
(-2.30)\end{array}$ & $\begin{array}{l}-0.331 \\
(-0.73)\end{array}$ & $\begin{array}{c}-12.41 * * * \\
(-3.21)\end{array}$ & $\begin{array}{c}-16.16 * * * \\
(-4.95)\end{array}$ & $\begin{array}{l}-8.335^{*} \\
(-1.94)\end{array}$ & $\begin{array}{l}-0.885 \\
(-0.75)\end{array}$ & $\begin{array}{l}-1.356 \\
(-1.15)\end{array}$ & $\begin{array}{l}-0.0219 \\
(-0.05)\end{array}$ \\
\hline Loan Loss Provisions & $\begin{array}{c}3.857 * * * \\
(3.91)\end{array}$ & $\begin{array}{c}3.754 * * * \\
(3.49)\end{array}$ & $\begin{array}{l}0.345 \\
(0.74)\end{array}$ & $\begin{array}{c}8.545^{* *} \\
(2.38)\end{array}$ & $\begin{array}{c}10.24 * * * \\
(3.02)\end{array}$ & $\begin{array}{c}18.11^{* * *} \\
(2.10)\end{array}$ & $\begin{array}{c}3.346 * * * \\
(3.34)\end{array}$ & $\begin{array}{c}2.516^{* * *} \\
(2.39)\end{array}$ & $\begin{array}{l}0.498 \\
(1.09)\end{array}$ \\
\hline
\end{tabular}


Table A.5. Determinants of Interest Rate, Exchange Rate, and Credit Risk Betas

Variable

Size

Capital Ratio

GDP Growth

Credit Derivatives

L. Credit Risk Beta

\section{Credit Risk Beta}

$N$

Adjusted-R2

$\operatorname{AR}(1)$

$\operatorname{AR}(2)$

Hansen J Statistic ( $p$-value)

Estimation Method

Note. Robustness check by using the change in the

window regression in the first-stage. We weight each observation by the inverse of the standard error of beta coefficients in the

variables. Heteroskedasticity-consistent standard errors are used and $t$ statistics are reported in parentheses. ${ }^{*} p<0.10,{ }^{* *} p<0.05,{ }^{* * *} p<0.01$.

first-stage estimation. The regressions included bank-specific fixed effects and yearly dummy
$i$ and the fourting
Small BHCs

$-0.0746$

$(-1.63)$

$-1.053$

0.462

$(-0.91)$

$-0.00339$

0.000253

(0.06)

$(-1.47)$

0.00754

(5.36)

(5.24)

$0.00933^{*}$

(2.03)

$2.060 * *$

$-0.00185$

(-0.19)

$-0.0195$

$-0.0259 *$

0.00499

$(-1.22)$

$(-1.85)$

(0.41)

(0.75)

$0.00114 \quad 0.00451^{* * *} \quad 0.00152$

$\begin{array}{lll}(1.33) & (2.78) & (1.23)\end{array}$

$-0.353 * * *$

0.00937

$0.00483 *$

(2.36)

$0.799^{* * * *}$

(9.76)

4672

0.000

0.057

0.266

0.141

0.121

2.055(0.561)

$0.415(0.813)$

GMM

FE

IV

GMM

sing data from Center for Research in Security Prices (CRSP) database and Federal Reserve monthly Statistical 
Table A.6. Determinants of Interest Rate, Exchange Rate, and Credit Risk Betas

\begin{tabular}{|c|c|c|c|c|c|c|c|c|c|}
\hline \multirow{2}{*}{$\begin{array}{l}\text { Variable } \\
\text { Panel A: Intere }\end{array}$} & \multicolumn{3}{|c|}{ Total BHCs } & \multicolumn{3}{|c|}{ Large BHCs } & \multicolumn{3}{|c|}{ Small BHCs } \\
\hline & ta (Foreign I & posure as Ins & Iment Variab & & & & & & \\
\hline \multirow{2}{*}{ Interest Margin } & $3.238 * * *$ & 0.880 & -1.097 & 7.394 & 6.870 & 22.92 & $2.823 * * *$ & 0.193 & $-29.45^{* *}$ \\
\hline & $(3.49)$ & $(0.43)$ & $(-0.53)$ & $(1.52)$ & $(1.28)$ & $(1.37)$ & $(3.08)$ & $(0.10)$ & $(-1.99)$ \\
\hline \multirow[t]{2}{*}{ C\&I Loans } & $-1.601 * * *$ & 0.166 & 1.132 & $16.65^{* * *}$ & $17.99 * * *$ & -0.767 & $-2.228 * * *$ & -1.725 & -1.748 \\
\hline & $(-3.38)$ & $(0.15)$ & $(1.21)$ & $(4.82)$ & $(4.64)$ & $(-0.71)$ & $(-4.76)$ & $(-1.63)$ & $(-0.88)$ \\
\hline \multirow[t]{2}{*}{ Mortgage Loans } & 0.191 & 0.652 & 1.076 & $11.64 * * *$ & $11.84 * * *$ & -0.820 & 0.125 & 0.287 & -0.0578 \\
\hline & $(0.71)$ & $(1.05)$ & $(1.56)$ & $(4.44)$ & $(4.33)$ & $(-0.72)$ & $(0.47)$ & $(0.48)$ & $(-0.07)$ \\
\hline \multirow[t]{2}{*}{ Other Loans } & -0.939 & 0.187 & 0.273 & 2.073 & 2.888 & -0.846 & -0.618 & -0.787 & 0.498 \\
\hline & $(-1.58)$ & $(0.12)$ & $(0.22)$ & $(0.70)$ & $(0.85)$ & $(-0.64)$ & $(-1.13)$ & $(-0.64)$ & $(0.27)$ \\
\hline \multirow[t]{2}{*}{ Domestic Deposits } & -0.446 & $1.792 * * *$ & -0.000112 & 1.771 & 1.226 & 0.0149 & $-1.280 * * *$ & 0.497 & -0.452 \\
\hline & $(-1.45)$ & $(2.90)$ & $(-0.00)$ & $(0.89)$ & $(0.58)$ & $(0.04)$ & $(-4.30)$ & $(0.86)$ & $(-0.42)$ \\
\hline \multirow[t]{2}{*}{ GAP Ratio } & $-0.00918^{* *}$ & $-0.00873 * *$ & $-0.0296 * * *$ & 0.00964 & $0.0175^{* *}$ & -0.000466 & -0.000455 & 0.0790 & 0.202 \\
\hline & $(-2.52)$ & $(-2.07)$ & $(-7.42)$ & $(1.50)$ & $(2.36)$ & $(-0.26)$ & $(-0.04)$ & $(1.57)$ & $(1.45)$ \\
\hline \multirow[t]{2}{*}{ Size } & $0.482 * * *$ & $0.713 * * *$ & 0.240 & $1.021 * *$ & $1.116^{* *}$ & -0.247 & $0.305 * * *$ & $0.272 *$ & 0.230 \\
\hline & $(6.52)$ & $(4.38)$ & $(0.81)$ & $(2.19)$ & $(2.30)$ & $(-0.98)$ & $(4.45)$ & $(1.90)$ & $(1.12)$ \\
\hline \multirow[t]{2}{*}{ Capital Ratio } & -0.0251 & 0.180 & -0.00290 & $13.21 * * *$ & $12.77 * *$ & -3.450 & -0.0916 & 0.0224 & -0.0198 \\
\hline & $(-0.24)$ & $(1.14)$ & $(-0.30)$ & $(2.59)$ & $(2.09)$ & $(-1.33)$ & $(-1.31)$ & $(0.53)$ & $(-0.10)$ \\
\hline \multirow[t]{2}{*}{ GDP Growth } & $-0.0177 * * *$ & $-0.0286 * *$ & 0.000267 & 0.00875 & 0.000190 & 0.00176 & $-0.0254 * * *$ & $-0.0679 * * *$ & 0.00890 \\
\hline & $(-2.80)$ & $(-2.08)$ & $(0.02)$ & $(0.29)$ & $(0.01)$ & $(0.17)$ & $(-3.96)$ & $(-4.68)$ & $(0.49)$ \\
\hline \multirow[t]{2}{*}{ Interest Rate Derivatives } & $0.117 * * *$ & $0.144 * * *$ & $0.299 *$ & $0.0910 * *$ & $0.0803^{*}$ & $0.0135^{* *}$ & 0.0792 & 0.0834 & 0.156 \\
\hline & $(3.71)$ & $(3.63)$ & $(1.75)$ & $(2.37)$ & $(1.75)$ & $(2.27)$ & $(1.43)$ & $(1.20)$ & $(1.48)$ \\
\hline \multirow[t]{2}{*}{ L.Interest Rate Risk Beta } & & & $0.421 * *$ & & & $1.068 * * *$ & & & $0.726^{* * *}$ \\
\hline & & & $(2.15)$ & & & $(99.45)$ & & & $(6.57)$ \\
\hline$N$ & 14128 & 6154 & 5476 & 792 & 744 & 762 & 13336 & 5410 & 4714 \\
\hline Adjusted-R2 & 0.106 & 0.0633 & & 0.460 & 0.461 & & 0.0985 & 0.0209 & \\
\hline $\operatorname{AR}(1)$ & & & 0.012 & & & 0.000 & & & 0.000 \\
\hline $\operatorname{AR}(2)$ & & & 0.606 & & & 0.149 & & & 0.642 \\
\hline Hansen J Statistic ( $p$-value) & & & $12.70(0.241)$ & & & $0.204(0.651)$ & & & $2.478(0.649)$ \\
\hline
\end{tabular}

Panel B: Exchange Rate Risk Beta (Basis Exposure as Instrument Variable) 
Table A.6. Determinants of Interest Rate, Exchange Rate, and Credit Risk Betas

\begin{tabular}{|c|c|c|c|c|c|c|c|c|c|}
\hline \multirow{2}{*}{$\begin{array}{l}\text { Variable } \\
\text { Assets in Foreign Currencies }\end{array}$} & \multicolumn{3}{|c|}{ Total BHCs } & \multicolumn{3}{|c|}{ Large BHCs } & \multicolumn{3}{|c|}{ Small BHCs } \\
\hline & $\begin{array}{l}-0.570 \\
(-0.55)\end{array}$ & $\begin{array}{l}-1.165 \\
(-0.97)\end{array}$ & $\begin{array}{l}-0.0998 \\
(-0.21)\end{array}$ & $\begin{array}{c}-5.007 * * * \\
(-4.14)\end{array}$ & $\begin{array}{c}-4.647 * * * \\
(-3.50)\end{array}$ & $\begin{array}{l}0.709 \\
(0.97)\end{array}$ & $\begin{array}{l}4.954 * * * \\
(5.02)\end{array}$ & $\begin{array}{c}4.665 * * * \\
(3.75)\end{array}$ & $\begin{array}{c}1.745^{* * *} \\
(2.95)\end{array}$ \\
\hline Foreign Exchange Deposits & $\begin{array}{l}0.820 \\
(1.30)\end{array}$ & $\begin{array}{l}0.212 \\
(0.24)\end{array}$ & $\begin{array}{l}0.508 \\
(0.70)\end{array}$ & $\begin{array}{c}0.0423 \\
(0.04)\end{array}$ & $\begin{array}{l}-0.689 \\
(-0.56)\end{array}$ & $\begin{array}{l}0.459 \\
(0.43)\end{array}$ & $\begin{array}{l}0.595 \\
(0.78)\end{array}$ & $\begin{array}{l}-0.954 \\
(-0.79)\end{array}$ & $\begin{array}{l}0.379 \\
(0.31)\end{array}$ \\
\hline Size & $\begin{array}{l}-0.0434 \\
(-0.96)\end{array}$ & $\begin{array}{l}-0.131 * \\
(-1.84)\end{array}$ & $\begin{array}{l}0.181^{*} \\
(1.84)\end{array}$ & $\begin{array}{l}0.150 \\
(1.16)\end{array}$ & $\begin{array}{r}0.0661 \\
(0.52)\end{array}$ & $\begin{array}{l}-0.113 \\
(-0.34)\end{array}$ & $\begin{array}{l}-0.0305 \\
(-0.64)\end{array}$ & $\begin{array}{l}-0.0948 \\
(-1.16)\end{array}$ & $\begin{array}{c}-0.301 * * * \\
(-2.83)\end{array}$ \\
\hline Capital Ratio & $\begin{array}{c}0.000939 \\
(0.06)\end{array}$ & $\begin{array}{c}-0.00753 \\
(-0.64)\end{array}$ & $\begin{array}{l}0.000281 \\
(0.23)\end{array}$ & $\begin{array}{l}-3.260 * \\
(-1.67)\end{array}$ & $\begin{array}{l}-3.341 \\
(-1.51)\end{array}$ & $\begin{array}{l}-0.625 \\
(-0.22)\end{array}$ & $\begin{array}{c}-0.00229 \\
(-0.16)\end{array}$ & $\begin{array}{c}-0.00716 \\
(-0.70)\end{array}$ & $\begin{array}{c}0.00317 \\
(1.19)\end{array}$ \\
\hline GDP Growth & $\begin{array}{c}0.00678 \\
(1.38)\end{array}$ & $\begin{array}{c}0.0120 * \\
(1.81)\end{array}$ & $\begin{array}{c}-0.00450 \\
(-0.60)\end{array}$ & $\begin{array}{c}-0.000443 \\
(-0.02)\end{array}$ & $\begin{array}{c}-0.00910 \\
(-0.48)\end{array}$ & $\begin{array}{c}0.00485 \\
(0.18)\end{array}$ & $\begin{array}{c}0.00886^{*} \\
(1.75)\end{array}$ & $\begin{array}{c}0.0220 * * * \\
(3.11)\end{array}$ & $\begin{array}{c}-0.00343 \\
(-0.67)\end{array}$ \\
\hline Exchange Rate Derivatives & $\begin{array}{c}0.600 * * * \\
(6.66)\end{array}$ & $\begin{array}{c}0.716^{* * * *} \\
(5.84)\end{array}$ & $\begin{array}{c}0.150 * * * \\
(4.30)\end{array}$ & $\begin{array}{c}0.693 * * * \\
(6.36)\end{array}$ & $\begin{array}{c}0.823 * * * \\
(5.53)\end{array}$ & $\begin{array}{c}0.0309 \\
(0.54)\end{array}$ & $\begin{array}{c}0.826 * * * \\
(4.82)\end{array}$ & $\begin{array}{c}0.905 * * * \\
(3.63)\end{array}$ & $\begin{array}{c}0.121 * * \\
(1.99)\end{array}$ \\
\hline L. Exchange Rate Risk Beta & & & $\begin{array}{c}1.119 * * * \\
(13.06)\end{array}$ & & & $\begin{array}{c}1.159 * * * \\
(4.81)\end{array}$ & & & $\begin{array}{c}-0.686^{* * *} \\
(-5.14)\end{array}$ \\
\hline$N$ & 14136 & 6160 & 5480 & 797 & 749 & 717 & 13339 & 5411 & 4763 \\
\hline Adjusted-R2 & 0.189 & 0.238 & & 0.441 & 0.462 & & 0.182 & 0.216 & \\
\hline $\operatorname{AR}(1)$ & & & 0.000 & & & 0.013 & & & 0.000 \\
\hline $\operatorname{AR}(2)$ & & & 0.115 & & & 0.180 & & & 0.300 \\
\hline Hansen J Statistic ( $p$-value) & & & $0.109(0.947)$ & & & $5.360(0.252)$ & & & $0.359(0.836)$ \\
\hline \multicolumn{10}{|c|}{ Panel C: Credit Risk Beta (Foreign Exposure as Instrument Variable) } \\
\hline Market Liquidity & $\begin{array}{c}-0.134 * * * \\
(-4.98)\end{array}$ & $\begin{array}{c}-0.127 * * * \\
(-2.98)\end{array}$ & $\begin{array}{c}-0.0164 \\
(-0.53)\end{array}$ & $\begin{array}{c}0.334 * * * \\
(3.39)\end{array}$ & $\begin{array}{c}0.314 * * * \\
(3.06)\end{array}$ & $\begin{array}{c}0.0754 \\
(0.44)\end{array}$ & $\begin{array}{c}-0.139 * * * \\
(-5.07)\end{array}$ & $\begin{array}{c}-0.165^{* * *} \\
(-3.69)\end{array}$ & $\begin{array}{c}-0.0228 \\
(-0.70)\end{array}$ \\
\hline Funding Liquidity & $\begin{array}{c}-0.181 * * * \\
(-3.25)\end{array}$ & $\begin{array}{c}-0.210^{* *} \\
(-2.34)\end{array}$ & $\begin{array}{c}-0.00533 \\
(-0.16)\end{array}$ & $\begin{array}{c}-1.093^{* * *} \\
(-4.37)\end{array}$ & $\begin{array}{c}-1.121 * * * \\
(-4.55)\end{array}$ & $\begin{array}{c}0.0974 \\
(0.42)\end{array}$ & $\begin{array}{c}-0.162 * * * \\
(-2.82)\end{array}$ & $\begin{array}{l}-0.107 \\
(-1.08)\end{array}$ & $\begin{array}{c}-0.0102 \\
(-0.29)\end{array}$ \\
\hline Non-Performing Loans & $\begin{array}{c}-1.871 * * * \\
(-9.93)\end{array}$ & $\begin{array}{c}-0.957 * * * \\
(-4.76)\end{array}$ & $\begin{array}{c}0.0487 \\
(0.46)\end{array}$ & $\begin{array}{c}-6.241^{* * * *} \\
(-7.14)\end{array}$ & $\begin{array}{c}-6.623 * * * \\
(-7.23)\end{array}$ & $\begin{array}{l}-6.953 \\
(-0.96)\end{array}$ & $\begin{array}{c}-1.858 * * * \\
(-9.98)\end{array}$ & $\begin{array}{c}-0.773 * * * \\
(-4.26)\end{array}$ & $\begin{array}{c}0.0400 \\
(0.38)\end{array}$ \\
\hline Loan Charge-Offs & $\begin{array}{l}-1.710 \\
(-1.48)\end{array}$ & $\begin{array}{c}-2.840 * * \\
(-2.38)\end{array}$ & $\begin{array}{l}-0.270 \\
(-0.61)\end{array}$ & $\begin{array}{c}-12.41 * * * \\
(-3.21)\end{array}$ & $\begin{array}{c}-16.13 * * * \\
(-4.94)\end{array}$ & $\begin{array}{l}-3.457 \\
(-0.44)\end{array}$ & $\begin{array}{l}-0.885 \\
(-0.75)\end{array}$ & $\begin{array}{l}-1.455 \\
(-1.23)\end{array}$ & $\begin{array}{c}-0.0364 \\
(-0.08)\end{array}$ \\
\hline Loan Loss Provisions & $\begin{array}{c}3.857 * * * \\
(3.91)\end{array}$ & $\begin{array}{c}3.860 * * * \\
(3.59)\end{array}$ & $\begin{array}{l}0.245 \\
(0.54)\end{array}$ & $\begin{array}{c}8.545^{* *} \\
(2.38)\end{array}$ & $\begin{array}{c}10.21 * * * \\
(3.01)\end{array}$ & $\begin{array}{l}9.859 \\
(0.93)\end{array}$ & $\begin{array}{c}3.346^{* * *} \\
(3.34)\end{array}$ & $\begin{array}{c}2.630 * * \\
(2.50)\end{array}$ & $\begin{array}{c}0.0364 \\
(0.08)\end{array}$ \\
\hline
\end{tabular}


Table A.6. Determinants of Interest Rate, Exchange Rate, and Credit Risk Betas

\begin{tabular}{|c|c|c|c|c|c|c|c|c|c|}
\hline Variable & & Total BHC & & & Large $\mathrm{BH}$ & & & Small BHCs & \\
\hline Size & $\begin{array}{c}-0.00759 \\
(-0.80)\end{array}$ & $\begin{array}{l}-0.0215 \\
(-1.43)\end{array}$ & $\begin{array}{c}-0.0329 * * * \\
(-3.77)\end{array}$ & $\begin{array}{c}0.0967 * * * \\
(3.55)\end{array}$ & $\begin{array}{c}0.126^{* * * *} \\
(4.16)\end{array}$ & $\begin{array}{c}-0.131 * * * \\
(-2.94)\end{array}$ & $\begin{array}{c}-0.00185 \\
(-0.19)\end{array}$ & $\begin{array}{l}-0.0138 \\
(-0.87)\end{array}$ & $\begin{array}{c}-0.0294 * * * \\
(-2.96)\end{array}$ \\
\hline Capital Ratio & $\begin{array}{c}0.00266 \\
(0.42)\end{array}$ & $\begin{array}{c}-0.00282 \\
(-0.72)\end{array}$ & $\begin{array}{c}0.000247 \\
(0.59)\end{array}$ & $\begin{array}{c}0.762 * \\
(1.95)\end{array}$ & $\begin{array}{l}0.454 \\
(0.90)\end{array}$ & $\begin{array}{c}-2.378^{*} \\
(-2.01)\end{array}$ & $\begin{array}{c}0.00499 \\
(0.57)\end{array}$ & $\begin{array}{c}0.00123 \\
(0.32)\end{array}$ & $\begin{array}{c}0.000314 \\
(0.64)\end{array}$ \\
\hline GDP Growth & $\begin{array}{c}0.000603 \\
(0.72)\end{array}$ & $\begin{array}{c}0.00215 \\
(1.44)\end{array}$ & $\begin{array}{c}0.00158 \\
(1.32)\end{array}$ & $\begin{array}{c}-0.00224 \\
(-1.03)\end{array}$ & $\begin{array}{c}-0.00342 \\
(-1.48)\end{array}$ & $\begin{array}{c}0.00158 \\
(0.64)\end{array}$ & $\begin{array}{c}0.00114 \\
(1.33)\end{array}$ & $\begin{array}{c}0.00448^{* * *} \\
(2.73)\end{array}$ & $\begin{array}{c}0.00183 \\
(1.35)\end{array}$ \\
\hline Credit Derivatives & $\begin{array}{c}0.0126 \\
(1.16)\end{array}$ & $\begin{array}{l}0.0179 \\
(1.42)\end{array}$ & $\begin{array}{c}0.00864 * \\
(1.69)\end{array}$ & $\begin{array}{c}0.0579 * * * \\
(5.36)\end{array}$ & $\begin{array}{c}0.0597 * * * \\
(5.36)\end{array}$ & $\begin{array}{c}0.156 * * * \\
(4.01)\end{array}$ & $\begin{array}{c}0.0183^{* *} \\
(2.26)\end{array}$ & $\begin{array}{c}0.00601 \\
(0.65)\end{array}$ & $\begin{array}{c}0.0115^{* * * *} \\
(3.13)\end{array}$ \\
\hline L. Credit Risk Beta & & & $\begin{array}{c}-0.381 * * * \\
(-4.05)\end{array}$ & & & $\begin{array}{c}0.0810 * * \\
(2.73)\end{array}$ & & & $\begin{array}{c}-0.436 * * * \\
(-3.48)\end{array}$ \\
\hline$N$ & 13359 & 5941 & 5316 & 737 & 707 & 685 & 12622 & 5234 & 4631 \\
\hline Adjusted-R2 & 0.0809 & 0.0771 & & 0.564 & 0.585 & & 0.0919 & 0.103 & \\
\hline $\operatorname{AR}(1)$ & & & 0.000 & & & 0.054 & & & 0.000 \\
\hline $\mathrm{AR}(2)$ & & & 0.137 & & & 0.427 & & & 0.324 \\
\hline Hansen J Statistic ( $p$-value) & & & $0.030(0.985)$ & & & $2.734(0.987)$ & & & $0.594(0.743)$ \\
\hline Estimation Method & FE & IV & GMM & $\mathrm{FE}$ & IV & GMM & $\mathrm{FE}$ & IV & GMM \\
\hline
\end{tabular}


Table A.7. Determinants of Interest Rate, Exchange Rate, and Credit Risk Betas, and Interaction Terms

Variable

Panel A: Interest Rate Risk Beta

Interest Margin

C\& I Loans

Mortgage Loans

Other Loans

Domestic Deposits

GAP Ratio

Size

Capital Ratio

Tier1 Ratio

GDP Growth

Interest Rate Derivatives

Capital Ratio * Interest Rate Derivatives

Tier1 Ratio * Interest Rate Derivatives

Size * Interest Rate Derivatives

SIFI * Interest Rate Derivatives

Interest Rate Derivatives for Trading

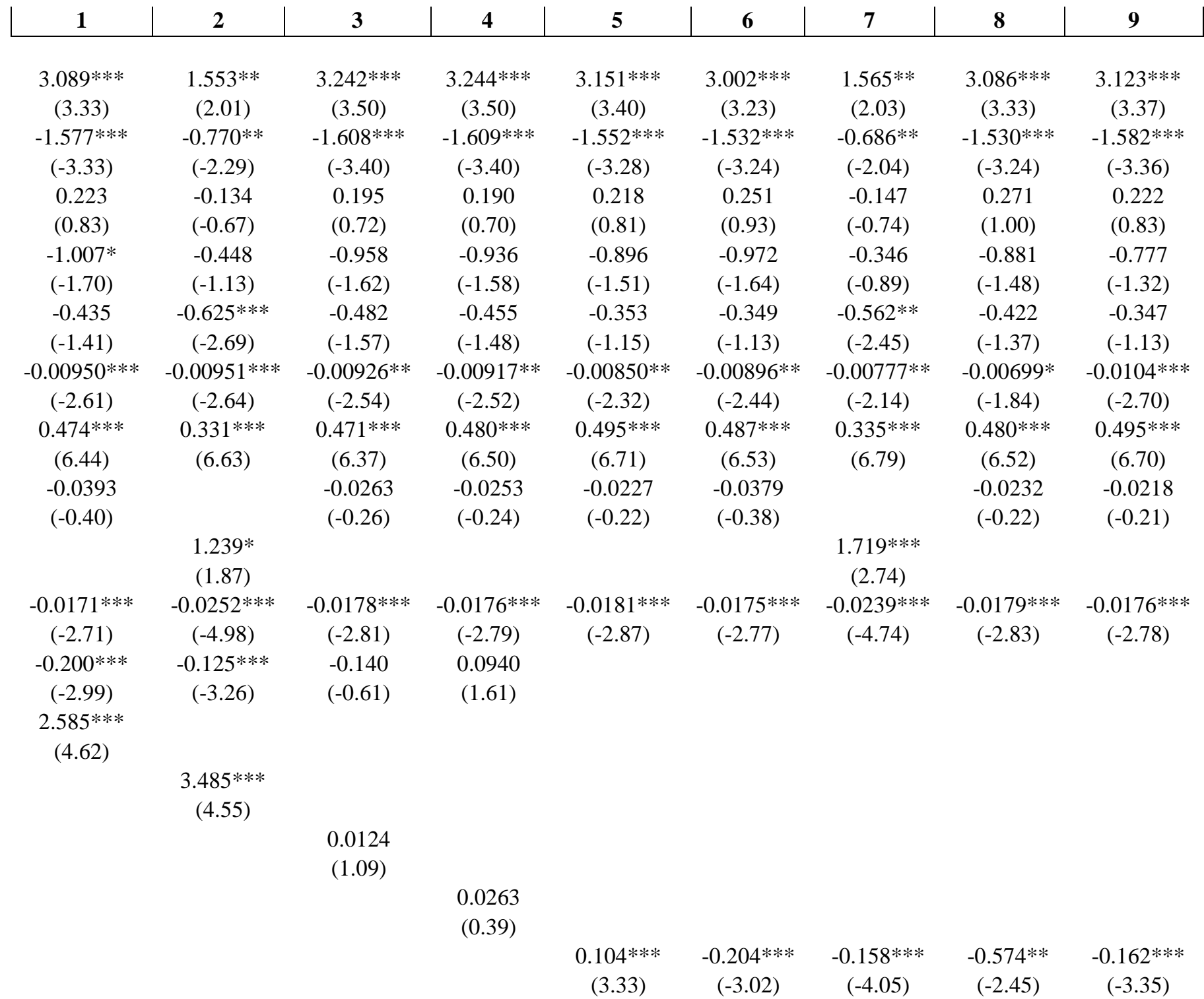


Table A.7. Determinants of Interest Rate, Exchange Rate, and Credit Risk Betas, and Interaction Terms

Variable

Interest Rate Derivatives for Hedging

$1 \quad \mid \quad 2$

Capital Ratio* Interest Rate Derivatives for Trading

Capital Ratio * Interest Rate Derivatives for Hedging

Tier1 Ratio * Interest Rate Derivatives for Trading

Tier1 Ratio * Interest Rate Derivatives for Hedging

Size * Interest Rate Derivatives for Trading

Size * Interest Rate Derivatives for Hedging

SIFI * Interest Rate Derivatives for Trading

$2 \quad$

$3 \quad 4$

4

SIFI * Interest Rate Derivatives for Hedging

\begin{tabular}{c|cc|c|c|}
$\mathbf{5}$ & $\mathbf{6}$ & $\mathbf{7}$ & $\mathbf{8}$ & $\mathbf{9}$ \\
\hline $0.374 * * *$ & -0.0472 & $1.405^{* * *}$ & -2.768 & $0.462 * * *$ \\
$(3.24)$ & $(-0.08)$ & $(3.45)$ & $(-1.64)$ & $(4.21)$ \\
& $2.526 * * *$ & & & \\
& $(4.40)$ & & & \\
& 3.135 & & & \\
& $(0.69)$ & & &
\end{tabular}

$N$

\begin{tabular}{l}
$N$ \\
Adjusted-R2 \\
\hline Panel B: Exchange Rate Risk Beta \\
Assets in Foreign Currencies
\end{tabular}

Assets in Foreign Currencies

Foreign Exchange Deposits

Size

Capital Ratio

Tier1 Ratio

GDP Growth

Exchange Rate Derivatives

(0.69)

$3.752 * * *$

(4.66)

$-10.12 * *$

$(-2.18)$

$0.0322 * * *$

(2.76)

$0.192^{*}$

(1.82)

$0.285^{* * *}$

(4.72)

$-0.908^{*}$

$(-1.68)$

$14128 \quad 17375$

17375
0.109

14128

14128

14132

14132

17379

0.111

14132

0.107

0.107

$-0.597$

$$
\text { (-0.57) }
$$

$-0.508$

$(-0.49)$

$-0.565$

$(-0.54)$

$-0.690$

$(-0.65)$

$-0.646$

$(-0.61)$

$-2.143 *$

$-1.745^{*}$

(-1.67)

0.936

(1.29)

(1.46)

(1.66)

0.953

(1.49)

$(-1.94)$

$1.169 *$

$1.136^{*}$

(1.85)

$-0.0575$

$-0.0490$

$-0.0421$

$-0.0490$

$(-1.08) \quad(-1.12)$

$(-0.98)$

$(-1.01)$

0.000389

0.000867

$0.000743 \quad 0.000472$

(0.02)

(0.03)

(0.06)

(0.05)

(0.03)

$(-1.23)$

$-0.200$

$(-0.47)$

$0.00670 \quad 0.00681$

(1.37)

(1.39)

0.00671

(1.37)

$-0.503$

0.00686

(1.40)

$0.826 * * *$

0.00673
$(1.38)$

0.00637
$(1.30)$

$(-0.87)$

0.00681

(1.39)

0.00662

(1.35)

0.00670

17
$-0.673 \quad-0.813$

$(-0.62) \quad(-0.75)$

$1.252 * * \quad 1.007$

(1.96) (1.60)

$-0.0570 \quad-0.0492$

$(-1.25) \quad(-1.08)$

$0.0000286 \quad 0.000746$

$(0.00) \quad(0.05)$ 
Table A.7. Determinants of Interest Rate, Exchange Rate, and Credit Risk Betas, and Interaction Terms

Variable

Capital Ratio * Exchange Rate Derivatives

Tier1 Ratio* Exchange Rate Derivatives

Size * Exchange Rate Derivatives

SIFI * Exchange Rate Derivatives

Exchange Rate Derivatives for Trading

Exchange Rate Derivatives for Hedging

Capital Ratio * ExchangeRate Derivatives for Trading

CapitalRatio * Exchange RateDerivatives forHedging

Tier1 Ratio * Exchange Rate Derivatives for Trading

Tier1 Ratio * Exchange Rate Derivatives for Hedging

Size * Exchange Rate Derivatives for Trading

Size * Exchange Rate Derivatives for Hedging

SIFI * Exchange Rate Derivatives for Trading

SIFI * Exchange Rate Derivatives for Hedging

\begin{tabular}{|c|c|}
$\mathbf{1}$ & $\mathbf{2}$ \\
\hline$(3.10)$ & $(4.12)$ \\
0.828 & \\
$(1.11)$ & \\
& -0.137 \\
& $(-0.08)$
\end{tabular}

$(-0.08)$

\subsection{6}

(1.62)

(-1.48)

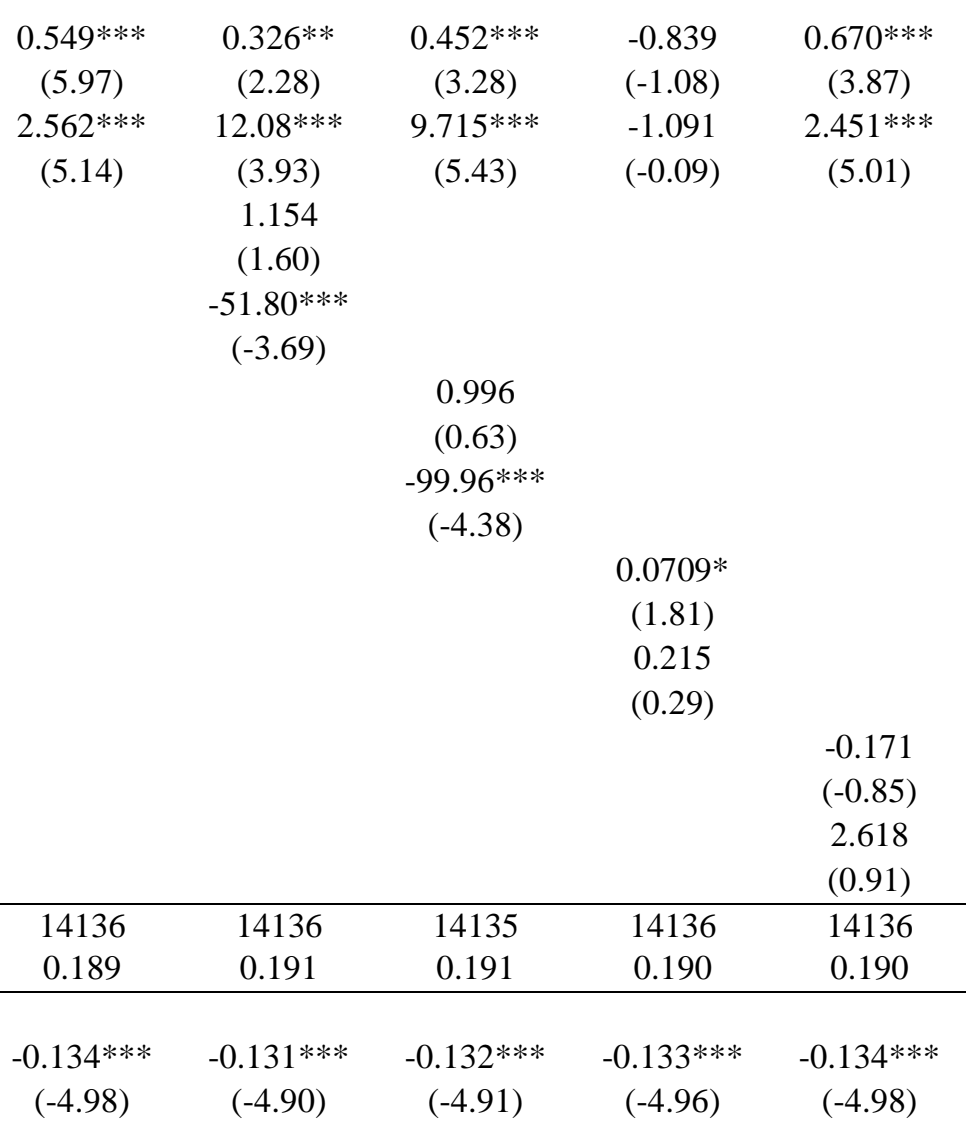

\begin{tabular}{|c|c|c|c|c|c|c|c|c|c|}
\hline$N$ & 14136 & 14135 & 14136 & 14136 & 14136 & 14136 & 14135 & 14136 & 14136 \\
\hline Adjusted-R2 & 0.189 & 0.189 & 0.189 & 0.189 & 0.189 & 0.191 & 0.191 & 0.190 & 0.190 \\
\hline \multicolumn{10}{|l|}{ Panel C: Credit Risk Beta } \\
\hline Market Liquidity & $\begin{array}{c}-0.132 * * * \\
(-4.91)\end{array}$ & $\begin{array}{c}-0.132 * * * \\
(-4.92)\end{array}$ & $\begin{array}{c}-0.130 * * * \\
(-4.94)\end{array}$ & $\begin{array}{c}-0.134 * * * \\
(-4.98)\end{array}$ & $\begin{array}{c}-0.134 * * * \\
(-4.98)\end{array}$ & $\begin{array}{c}-0.131 * * * \\
(-4.90)\end{array}$ & $\begin{array}{c}-0.132 * * * \\
(-4.91)\end{array}$ & $\begin{array}{c}-0.133 * * * \\
(-4.96)\end{array}$ & $\begin{array}{c}-0.134 * * * \\
(-4.98)\end{array}$ \\
\hline
\end{tabular}


Table A.7. Determinants of Interest Rate, Exchange Rate, and Credit Risk Betas, and Interaction Terms

\section{Variable}

Funding Liquidity

Non-Performing Loans

Loan Charge-Offs

Loan Loss Provisions

Size

Capital Ratio

Tier1 Ratio

GDP Growth

Credit Derivatives

Capital Ratio * Credit Derivatives

Tier1 Ratio * Credit Derivatives

Size $*$ Credit Derivatives

SIFI * Credit Derivatives

Gross Credit Protection

Net Credit Protection Bought

Capital Ratio * Gross Credit Protection

Capital Ratio * Net Credit Protection Bought

\begin{tabular}{|c|c|c|c|c|c|c|c|c|}
\hline 1 & 2 & 3 & 4 & 5 & 6 & 7 & 8 & 9 \\
\hline $\begin{array}{c}-0.184 * * * \\
(-3.29)\end{array}$ & $\begin{array}{c}-0.183 * * * \\
(-3.28)\end{array}$ & $\begin{array}{c}-0.108 * * \\
(-2.16)\end{array}$ & $\begin{array}{c}-0.181 * * * \\
(-3.24)\end{array}$ & $\begin{array}{c}-0.182 * * * \\
(-3.25)\end{array}$ & $\begin{array}{c}-0.187 * * * \\
(-3.35)\end{array}$ & $\begin{array}{c}-0.185 * * * \\
(-3.30)\end{array}$ & $\begin{array}{c}-0.181 * * * \\
(-3.24)\end{array}$ & $\begin{array}{c}-0.181 * * * \\
(-3.24)\end{array}$ \\
\hline $\begin{array}{c}-1.872 * * * \\
(-9.94)\end{array}$ & $\begin{array}{c}-1.875^{* * *} \\
(-10.06)\end{array}$ & $\begin{array}{c}-1.319 * * * \\
(-6.91)\end{array}$ & $\begin{array}{c}-1.871 * * * \\
(-9.93)\end{array}$ & $\begin{array}{c}-1.871 * * * \\
(-9.93)\end{array}$ & $\begin{array}{c}-1.872 * * * \\
(-9.94)\end{array}$ & $\begin{array}{c}-1.873 * * * \\
(-10.05)\end{array}$ & $\begin{array}{c}-1.872 * * * \\
(-9.93)\end{array}$ & $\begin{array}{c}-1.872 * * * \\
(-9.93)\end{array}$ \\
\hline $\begin{array}{l}-1.656 \\
(-1.43)\end{array}$ & $\begin{array}{l}-1.675 \\
(-1.45)\end{array}$ & $\begin{array}{l}0.204 \\
(0.20)\end{array}$ & $\begin{array}{l}-1.712 \\
(-1.48)\end{array}$ & $\begin{array}{l}-1.710 \\
(-1.48)\end{array}$ & $\begin{array}{l}-1.655 \\
(-1.43)\end{array}$ & $\begin{array}{l}-1.663 \\
(-1.44)\end{array}$ & $\begin{array}{l}-1.714 \\
(-1.48)\end{array}$ & $\begin{array}{l}-1.715 \\
(-1.48)\end{array}$ \\
\hline $\begin{array}{c}3.807 * * * \\
(3.86)\end{array}$ & $\begin{array}{c}3.820 * * * \\
(3.87)\end{array}$ & $\begin{array}{c}2.177 * * \\
(2.37)\end{array}$ & $\begin{array}{c}3.859 * * * \\
(3.91)\end{array}$ & $\begin{array}{c}3.857 * * * \\
(3.91)\end{array}$ & $\begin{array}{c}3.804 * * * \\
(3.85)\end{array}$ & $\begin{array}{c}3.804 * * * \\
(3.85)\end{array}$ & $\begin{array}{c}3.858 * * * \\
(3.91)\end{array}$ & $\begin{array}{c}3.864 * * * \\
(3.91)\end{array}$ \\
\hline $\begin{array}{c}-0.00763 \\
(-0.80)\end{array}$ & $\begin{array}{c}-0.00796 \\
(-0.79)\end{array}$ & $\begin{array}{c}0.00684 \\
(0.74)\end{array}$ & $\begin{array}{c}-0.00757 \\
(-0.79)\end{array}$ & $\begin{array}{c}-0.00760 \\
(-0.80)\end{array}$ & $\begin{array}{c}-0.00819 \\
(-0.86)\end{array}$ & $\begin{array}{c}-0.00798 \\
(-0.80)\end{array}$ & $\begin{array}{c}-0.00739 \\
(-0.77)\end{array}$ & $\begin{array}{c}-0.00758 \\
(-0.80)\end{array}$ \\
\hline $\begin{array}{c}0.00289 \\
(0.45)\end{array}$ & & $\begin{array}{c}-0.00359 \\
(-1.13)\end{array}$ & $\begin{array}{c}0.00266 \\
(0.42)\end{array}$ & $\begin{array}{c}0.00266 \\
(0.42)\end{array}$ & $\begin{array}{c}0.00300 \\
(0.46)\end{array}$ & & $\begin{array}{c}0.00270 \\
(0.43)\end{array}$ & $\begin{array}{c}0.00265 \\
(0.42)\end{array}$ \\
\hline & $\begin{array}{c}-0.0197 \\
(-0.26)\end{array}$ & & & & & $\begin{array}{c}-0.0176 \\
(-0.23)\end{array}$ & & \\
\hline $\begin{array}{c}0.000558 \\
(0.67)\end{array}$ & $\begin{array}{c}0.000629 \\
(0.76)\end{array}$ & $\begin{array}{c}-0.000365 \\
(-0.48)\end{array}$ & $\begin{array}{c}0.000603 \\
(0.72)\end{array}$ & $\begin{array}{c}0.000602 \\
(0.72)\end{array}$ & $\begin{array}{c}0.000518 \\
(0.62)\end{array}$ & $\begin{array}{c}0.000609 \\
(0.73)\end{array}$ & $\begin{array}{c}0.000595 \\
(0.71)\end{array}$ & $\begin{array}{c}0.000595 \\
(0.71)\end{array}$ \\
\hline
\end{tabular}

$-0.00697$

$(-0.48)$

$\begin{array}{ccccc}0.0122 & 0.164 * * * & 0.184 * * * & 0.399 & 0.00891 \\ (1.14) & (2.88) & (2.62) & (1.10) & (0.67) \\ 0.0234 & 3.405 * * & 2.064 & -1.952 & 0.391 \\ (0.31) & (2.12) & (1.38) & (-0.84) & (0.96) \\ & -1.040^{* *} & & & \\ & (-2.55) & & & \\ & -28.41 * * & & & \\ & (-2.11) & & \end{array}$


Table A.7. Determinants of Interest Rate, Exchange Rate, and Credit Risk Betas, and Interaction Terms

Variable

$1 \quad 2$

Tier1 Ratio * Gross Credit Protection

$2+3$

3

4

Tier1 Ratio * Net Credit Protection Bought

Size * Gross Credit Protection

$-0.0182$

Size * Net Credit Protection Bought

$(-1.05)$

0.0937

(0.85)

SIFI * Gross Credit Protection

0.00295

(0.17)

SIFI * Net Credit Protection Bought

$-0.385$

$(-0.93)$

$$
N
$$

13358

10921

13359

13359

13359

13358

0.0821

13359

13359

Adjusted-R2

FE

FE

$\mathrm{FE}$

$\mathrm{FE}$

FE

$\mathrm{FE}$

FE

FE

FE

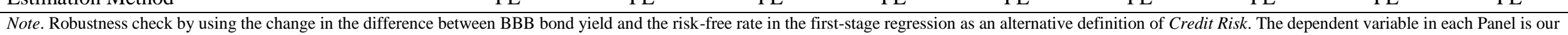

estimates of risk beta of each BHC $i$ at the start time $t$ of four-year rolling window regression in the first-stage. We weight each observation by the inverse of the standard error of beta coefficients in the first-stage estimation. The regressions included bank-specific fixed effects and yearly dummy variables. Heteroskedasticity-consistent standard errors are used and $t$ statistics are reported in parentheses. ${ }^{*} p<0.10,{ }^{* * *} p<0.05,{ }^{* * * *} p<0.01$.

Source: Financial Statements data from FR Y-9C; Risk betas are computed from the four-factor model using data from Center for Research in Security Prices (CRSP) database and Federal Reserve monthly Statistical Releases. 
Table A.8. Impact of Financial Crisis on the Determinants of Interest Rate, Exchange Rate, and Credit Risk Betas

Variable

Panel A: Interest Rate Risk Beta

Interest Margin

\section{C\&I Loans}

Mortgage Loans

Other Loans

Domestic Deposits

GAP Ratio

Size

Capital Ratio

GDP Growth

Interest Rate Derivatives

Crisis

Crisis * Interest Rate Derivatives

Interest Rate Derivatives for Trading

Interest Rate Derivatives for Hedging

Crisis * Interest Rate Derivatives for Trading

Crisis * Interest Rate Derivatives for Hedging

\begin{tabular}{|c|c|c|c|}
\hline 1 & 2 & 3 & 4 \\
\hline $\begin{array}{c}5.054 * * * \\
(5.25) \\
-1.523 * * * \\
(-3.21) \\
0.218 \\
(0.80) \\
-0.844 \\
(-1.42) \\
-0.571 * \\
(-1.86) \\
-0.00936 * * \\
(-2.50) \\
0.482 * * * \\
(6.53) \\
-0.0252 \\
(-0.24) \\
-0.0175 * * * \\
(-2.79) \\
0.117 * * * \\
(3.76) \\
-0.457 * * * \\
(-9.61)\end{array}$ & $\begin{array}{c}5.050 * * * \\
(5.25) \\
-1.527 * * * \\
(-3.22) \\
0.227 \\
(0.84) \\
-0.838 \\
(-1.41) \\
-0.583^{*} \\
(-1.90) \\
-0.00939 * * \\
(-2.51) \\
0.476 * * * \\
(6.45) \\
-0.0265 \\
(-0.26) \\
-0.0175 * * * \\
(-2.77) \\
0.109 * * * \\
(3.58) \\
-0.462 * * * \\
(-9.71) \\
0.0160 \\
(1.32)\end{array}$ & $\begin{array}{c}4.956 * * * \\
(5.15) \\
-1.478 * * * \\
(-3.12) \\
0.245 \\
(0.91) \\
-0.804 \\
(-1.35) \\
-0.481 \\
(-1.57) \\
-0.00871 * * \\
(-2.32) \\
0.495 * * * \\
(6.72) \\
-0.0229 \\
(-0.22) \\
-0.0179 * * * \\
(-2.85)\end{array}$ & $\begin{array}{c}4.897 * * * \\
(5.12) \\
-1.576 * * * \\
(-3.34) \\
0.412 \\
(1.52) \\
-0.660 \\
(-1.13) \\
-0.518 * \\
(-1.68) \\
-0.00597 \\
(-1.59) \\
0.469 * * * \\
(6.52) \\
-0.0289 \\
(-0.29) \\
-0.0167 * * * \\
(-2.66)\end{array}$ \\
\hline $\begin{array}{l}14128 \\
0.108 \\
\end{array}$ & $\begin{array}{l}14128 \\
0.108 \\
\end{array}$ & $\begin{array}{l}14132 \\
0.108 \\
\end{array}$ & $\begin{array}{l}14132 \\
0.116 \\
\end{array}$ \\
\hline $\begin{array}{c}-0.554 \\
(-0.53) \\
0.831\end{array}$ & $\begin{array}{c}-0.513 \\
(-0.49) \\
0.848\end{array}$ & $\begin{array}{c}-0.631 \\
(-0.59) \\
0.950\end{array}$ & $\begin{array}{c}-0.498 \\
(-0.46) \\
1.020\end{array}$ \\
\hline
\end{tabular}


Table A.8. Impact of Financial Crisis on the Determinants of Interest Rate, Exchange Rate, and Credit Risk Betas

Variable

Size

Capital Ratio

GDP Growth

Exchange Rate Derivatives

Crisis

Crisis * Exchange Rate Derivatives

Exchange Rate Derivatives for Trading

Exchange Rate Derivatives for Hedging

Crisis * Exchange Rate Derivatives for Trading

Crisis * Exchange Rate Derivatives for Hedging

\begin{tabular}{lc}
\hline$N$ & \\
Adjusted-R2 & 14136 \\
\hline Panel C: Credit Risk Beta & 0.190 \\
Market Liquidity & $-0.121^{* * * *}$ \\
& $(-4.49)$ \\
Funding Liquidity & $-0.130^{* *}$ \\
& $(-2.30)$ \\
Non-Performing Loans & $-1.894^{* * *}$ \\
& $(-9.93)$ \\
Loan Charge-Offs & -1.774 \\
& $(-1.53)$ \\
Loan Loss Provisions & $3.725 * * *$ \\
Size & $(3.77)$ \\
& -0.0105 \\
Capital Ratio & $(-1.11)$ \\
GDP Growth & 0.00134 \\
& $(0.24)$ \\
& 0.000520 \\
& $(0.63)$
\end{tabular}

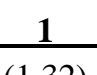

$-0.0486$

$(-1.07)$

$-0.0000902$

$(-0.01)$

$0.599 * * *$

(6.64)

$0.160 * * *$

(4.23)

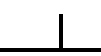

1

2

$-0.0501$

(-1.10)

$-0.000312$

$(-0.02)$
0.00663

(1.36)

$0.581 * * *$
$(6.33)$

$0.158 * * *$

(4.18)

0.0264

(0.78)

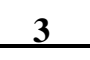

(1.52)

$-0.0545$

(-1.20)

$-0.000312$

$(-0.02)$

0.00665

(1.36)

$0.163 * * *$

(4.31)

$0.548 * * *$

(5.94)

(5.33)

1

4

(1.63)

$-0.0562$

(-1.24)

$-0.000790$

$(-0.05)$

0.00655

(1.34)

.164***

(4.32)

$.527^{* * * *}$

(5.62)

(5.37)

0.0488

(1.36)

$-2.889 *$

(-1.71)

14136
0.190

$121 * * * \quad-0.121 * * *$

$(-4.49)$
$-0.130 * *$

$(-2.29)$

$-1.894 * * *$

(-9.93)

$-1.774$

$(-1.53)$

$3.726 * * *$

(3.77)

$-0.0105$

$(-1.11)$

0.00133

$(0.23)$

0.000533

(0.64)
$-0.121 * * *$

(-4.49)

$-0.130 * *$

$(-2.29)$
$-1.894 * * *$

(-9.93)

$-1.774$

$(-1.53)$
$3.726 * * *$

(3.77)

$-0.0105$

$(-1.11)$

0.00133

(0.23)

0.000531

(0.64) 
Table A.8. Impact of Financial Crisis on the Determinants of Interest Rate, Exchange Rate, and Credit Risk Betas

Variable

Credit Derivatives

Crisis

Crisis * Credit Derivatives

Gross Credit Protection

Net Credit Protection Bought

Crisis * Gross Credit Protection

\begin{tabular}{cccc|}
$\mathbf{1}$ & $\mathbf{2}$ & $\mathbf{3}$ & $\mathbf{4}$ \\
\hline 0.0114 & 0.00501 & & \\
$(1.12)$ & $(0.61)$ & & $0.0965^{* * * *}$ \\
$0.0967^{* * *}$ & $0.0965^{* * *}$ & $0.0967 * * *$ & $(12.34)$ \\
$(12.37)$ & $(12.35)$ & $(12.37)$ & \\
& 0.00766 & & 0.00478 \\
& $(1.26)$ & 0.0109 & $0.59)$ \\
& & $(1.08)$ & 0.0200 \\
& & 0.0297 & $(0.37)$ \\
& & $(0.52)$ & 0.00740 \\
& & & $(1.17)$ \\
& & & 0.00642 \\
& & & $0.07)$ \\
\hline 13359 & 13359 & 13359 & 13359 \\
FE & & 0.0891 & 0.0892 \\
\hline
\end{tabular}

FE
FE
Crisis * Net Credit Protection Bought

Estimation Method

Note. Robustness check by using the change in the difference between BBB bond yield and the risk-free rate in the first-stage regression as an alternative definition of Credit Risk. The dependent variable in each
Panel is our estimates of risk beta of each BHC $i$ at the start time $t$ of four-year rolling window regression in the first-stage. We weight each observation by the inverse of the standard error of beta coefficients in the first-stage estimation. The regressions included bank-specific fixed effects and yearly dummy variables. Heteroskedasticity-consistent standard errors are used and $t$ statistics are reported in parentheses. ${ }^{*} p<0.10,{ }^{* *} p<$ $0.05, \stackrel{* * * *}{p}<0.01$

Source: Financial Statements data from FR Y-9C; Risk betas are computed from the four-factor model using data from Center for Research in Security Prices (CRSP) database and Federal Reserve monthly Statistical Releases. 
Table A.9. The Impact of Financial Derivatives on Scaled Idiosyncratic Risk $\left(\boldsymbol{I}-\boldsymbol{R}^{2}\right)$

Variable

Idiosyncratic Risk

Size

Capital Ratio

GDP Growth

Interest Rate Derivatives

Exchange Rate Derivatives

Credit Derivatives

Total Financial Derivatives

Total BHCs

$\begin{array}{cc}0.0492 * * * & 0.0812 * * * \\ (9.91) & (10.26) \\ 0.00353 & 0.00674 \\ (0.65) & (1.18) \\ 0.00101 * & 0.00218 * * \\ (1.83) & (2.52) \\ -0.00891 * * * & -0.00855 * * * \\ (-5.02) & (-4.63) \\ -0.0586 * * * & -0.0742 * * * \\ (-5.08) & (-5.81) \\ 0.0313 * * * & 0.0211^{* *} \\ (3.15) & (2.06)\end{array}$

Financial Derivatives for Trading

Financial Derivatives for Hedging
Large BHCs

Small BHCs

$\begin{array}{cccc}0.0506 * * * & 0.0506^{* * *} & 0.102 * * * & 0.108 * * * \\ (10.13) & (10.13) & (5.43) & (5.46) \\ 0.00354 & 0.00354 & 0.442 * * & 0.147 \\ (0.64) & (0.64) & (2.01) & (0.53) \\ 0.00106^{*} & 0.00106^{*} & 0.00581^{*} & 0.00393 \\ (1.92) & (1.92) & (1.91) & (1.31) \\ & & -0.00896^{* * *} & -0.00821^{* * *} \\ & & (-6.24) & (-5.48) \\ & & -0.0668 * * * & -0.0765 * * * \\ & & (-6.25) & (-6.02) \\ & & 0.00823 & 0.00748 \\ & & (0.77) & (0.71)\end{array}$

\section{$-0.00696 * * *$}

$(-4.35)$

$0.116 * * *$
$(6.61)$
$0.662 * * *$
$(2.88)$
$0.00632 * *$

$0.0427 * * *$ (8.37)

0.00298

(0.63)

$0.00632 * *$

0.000292

$0.0739 * * *$

(8.59)

0.00636

(1.21)

0.000829

$\begin{array}{cr}(0.53) & (0.99) \\ 0.00962 * * & 0.0129\end{array}$

(2.02)

$-0.194 * * * \quad-0.199 * * *$
(-4.94)
$(-4.87)$

$-0.0510 * * * \quad-0.0779 * * *$

$(-3.32) \quad(-3.68)$
$0.0436 * * *$

(8.48)

0.00273

$(0.59)$

0.000353

(0.64)

\begin{tabular}{|c|c|c|c|c|c|c|c|c|c|c|}
\hline 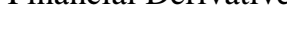 & & & & $(-0.60)$ & & & $(-2.50)$ & & & $(0.17)$ \\
\hline$N$ & 14263 & 6189 & 14263 & 14263 & 797 & 749 & 797 & 13466 & 5440 & 13466 \\
\hline Adjusted-R2 & 0.367 & 0.501 & 0.364 & 0.364 & 0.654 & 0.670 & 0.639 & 0.353 & 0.482 & 0.348 \\
\hline Estimation Method & $\mathrm{FE}$ & IV & $\mathrm{FE}$ & FE & $\mathrm{FE}$ & IV & FE & FE & IV & $\mathrm{FE}$ \\
\hline
\end{tabular}

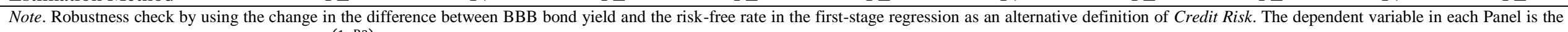

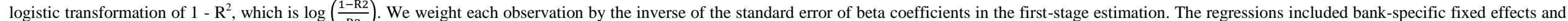
yearly dummy variables. Heteroskedasticity-consistent standard errors are used and $t$ statistics are reported in parentheses. ${ }^{*} p<0.10,{ }^{* *} p<0.05,{ }^{* * *} p<0.01$.

Source: Financial Statements data from FR Y-9C; Risk betas are computed from the four-factor model using data from Center for Research in Security Prices (CRSP) database and Federal Reserve monthly Statistical Releases. 
Table A.10. The Impact of Financial Derivatives on Market Risk Beta

Variable

Market Risk Beta

Size

Capital Ratio

GDP Growth

Interest Rate Derivatives

Exchange Rate Derivatives

Credit Derivatives

Adjusted-R2

Estimation Method

Total Sample

(12.77)

0.0245

$0.254 * * *$

$(1.04)$

$0.00395^{*}$

$(1.80)$

$0.0399 * * *$

(5.14)

$-0.444 * * *$

$(-11.34)$

0.00826

$(0.17)$

14263

0.429

FE

(7.67)

0.0199

(1.21)

$-0.00205$

$(-0.59)$

$0.0459 * * *$

(5.50)

$-0.505 * * *$

$(-10.37)$

$-0.0282$

$(-0.54)$

6189

6189
0.518

IV

(3.97)

$2.638 * *$

(2.36)

$0.0216^{*}$

(1.84)

$0.0557 * * *$

(7.42)

$-0.599 * * *$

$(-13.24)$

$-0.142 * *$

$(-2.53)$

797

0.665
FE

Large BHCs

Small BHCs

作 estimation. The regressions included bank-specific fixed effects and yearly dummy variables. Heteroskedasticity-consistent standard errors are used and $t$ statistics are reported in parentheses. ${ }^{*} p<0.10,{ }^{* * *} p<0.05,{ }^{* * *} p<0.01$.

Source: Financial Statements data from FR Y-9C; Risk betas are computed from the four-factor model using data from Center for Research in Security Prices (CRSP) database and Federal Reserve monthly Statistical Releases. 


\section{Appendix B: Chapter 2}

\section{Table B.1. The Construction of Variables and Data Source}

\begin{tabular}{|c|c|}
\hline Variable & efinition \\
\hline $\operatorname{lnGL}$ & The logarithm of gross loans \\
\hline TIER1 & Tier 1 capital to total risk-weighted assets ratio \\
\hline TIER2 & Tier 2 capital ratio, computed by subtracting Tier 1 capital ratio from total capital ratio \\
\hline TCD & The ratio of total customer deposits to total assets \\
\hline DEP & The ratio of interbank deposit to total assets \\
\hline TA & The logarithm of total assets representing the proxy for the size, $\mathrm{TA}=\log$ (total asset) \\
\hline LLP & The ratio of loan loss provision to gross loans \\
\hline FA & The ratio of fixed asset to total assets representing proxy for tangibility of bank assets \\
\hline TAXTA & The ratio of tax to the bank size, TAXTA $=$ Tax $/ \mathrm{TA}$ \\
\hline ROA & The ratio of net income to average total assets in recent two years, $\mathrm{ROA}_{\mathrm{t}}=2 * \mathrm{Net}_{\text {income }} / \mathrm{f}$ \\
\hline$\delta$ & A dummy variable for crisis, which takes value of 1 for period 2008-2010 and 0 otherwise \\
\hline GDP growth & Annual growth rate of GDP at market prices based on constant local currency \\
\hline Interest rate & The interest rate charged by banks on loans to prime customers. \\
\hline HHI & Herfindahl-Hirschman Index, defined as the sum of the squares of the market shares of asset o \\
\hline COMPTIER1 & The tier 1 capital ratio of the competitor banks: COMPTIER $1=\sum_{k \neq i}^{N_{j}} \operatorname{TIER} 1_{k, j} \frac{a_{k, j}}{A_{j}}$, Where $N_{j}$ \\
\hline & $a_{k, j}$ are the total assets of bank $k$ in country $j$, and $A_{j}=\sum_{k}^{N_{j}} a_{k, j}$ are the total assets of banks in \\
\hline Overall capital & Measures the extent of regulatory requirements regarding the amount of capital banks must ho \\
\hline & eposit insu \\
\hline Deposit insurance & eposit insu \\
\hline Commercial dummy & Takes value of 1 if the bank is a commercial bank and 0 otherwise \\
\hline Savings dummy & Takes value of 1 if the bank is a saving bank and 0 otherwise \\
\hline Government dummy & Takes value of 1 if the bank is a government-owned bank and 0 otherwise \\
\hline Foreign dummy & Takes value of 1 if the bank is a foreign-owned bank and 0 otherwise \\
\hline Subsidiary dummy & Takes value of 1 if the bank is a subsidiary bank and 0 otherwise \\
\hline Bail-out probability & Bail-out probabilities, based on the support ratings provided by the rating agency Fitch/IBCA. \\
\hline
\end{tabular}


Table B.2. Number of Observations in Sample Countries, 2001-2010

\begin{tabular}{|c|c|c|c|c|c|c|c|}
\hline Country & Sample & Country & Sample & Country & Sample & Country & Sample \\
\hline Afghanistan & 99 & Ecuador & 748 & Liberia & 44 & Saint Lucia & 44 \\
\hline Albania & 165 & Egypt & 440 & Libyan Arab Jamahir & 121 & San Marino & 66 \\
\hline Algeria & 220 & El Salvador & 220 & Liechtenstein & 33 & Sao Tome \& Principe & 33 \\
\hline Andorra & 44 & Equatorial Guinea & 22 & Lithuania & 165 & Saudi Arabia & 22 \\
\hline Angola & 143 & Eritrea & 22 & Luxembourg & 1848 & Senegal & 143 \\
\hline Anguilla & 22 & Estonia & 154 & Macau & 110 & Serbia & 737 \\
\hline Argentina & 1463 & Fiji & 44 & Madagascar & 66 & Sierra Leone & 121 \\
\hline Armenia & 275 & Finland & 341 & Malawi & 99 & Singapore & 495 \\
\hline Australia & 1155 & France & 5137 & Malaysia & 583 & Slovakia & 385 \\
\hline Austria & 3729 & Gabon & 55 & Maldives & 22 & Slovenia & 451 \\
\hline Azerbaijan & 231 & Gambia & 77 & Mali & 121 & South Africa & 539 \\
\hline Bahamas & 374 & Georgia Rep. Of & 165 & Malta & 132 & Spain & 3993 \\
\hline Bangladesh & 396 & Ghana & 429 & Mauritius & 264 & St. Kitts and Nevis & 22 \\
\hline Barbados & 55 & Gibraltar & 22 & Mexico & 693 & St. Vincent & 22 \\
\hline Belarus & 385 & Greece & 517 & Micronesia, Federal & 11 & Sudan & 165 \\
\hline Belgium & 1188 & Grenada & 33 & Moldova Rep. Of & 308 & Supranational & 22 \\
\hline Belize & 55 & Guatemala & 561 & Monaco & 44 & Suriname & 22 \\
\hline Benin & 99 & Guinea & 55 & Mongolia & 99 & Swaziland & 66 \\
\hline Bhutan & 33 & Guinea-Bissau & 11 & Montenegro & 121 & Sweden & 1485 \\
\hline Bolivia & 187 & Guyana & 33 & Morocco & 187 & Switzerland & 6237 \\
\hline Bosnia-Herzegovina & 418 & Haiti & 33 & Mozambique & 209 & Syria & 165 \\
\hline Botswana & 165 & Honduras & 440 & Myanmar Union of & 44 & Taiwan & 605 \\
\hline Brazil & 2244 & Hong Kong & 946 & Namibia & 154 & Tajikistan & 55 \\
\hline Brunei Darussalam & 11 & Hungary & 528 & Nauru & 44 & Tanzania & 539 \\
\hline Bulgaria & 418 & Iceland & 396 & Nepal & 275 & Thailand & 341 \\
\hline
\end{tabular}


Table B.2. Number of Observations in Sample Countries, 2001-2010

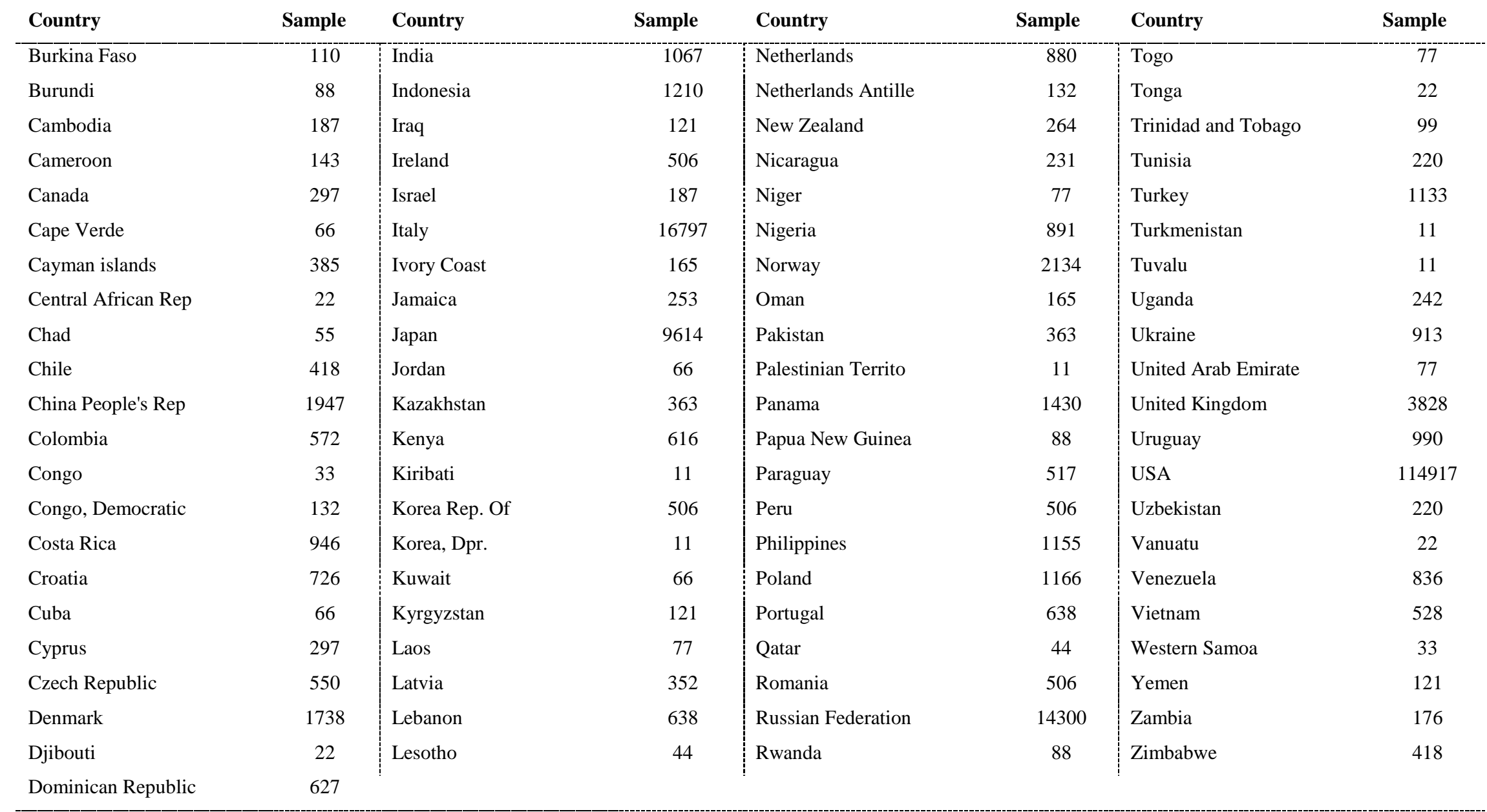

Note. Summary of the observations in each country in the total sample for 2001-2010 
Table B.3. Estimation Result of Commercial Banks

\begin{tabular}{|c|c|c|c|c|c|c|c|c|c|c|c|c|c|c|c|}
\hline & (1) & (2) & (3) & (4) & (5) & (6) & (7) & (8) & (9) & (10) & (11) & (12) & (13) & (14) & (15) \\
\hline $\begin{array}{l}\text { Bank specific variables } \\
\text { Intercept }\end{array}$ & $\begin{array}{c}2.929^{* * *} \\
(5.56)\end{array}$ & $\begin{array}{c}3.189^{* * *} \\
(5.68)\end{array}$ & $\begin{array}{l}2.924^{* * * *} \\
(5.53)\end{array}$ & $\begin{array}{c}2.997^{* * *} \\
(5.23)\end{array}$ & $\begin{array}{c}2.432^{* * *} \\
(3.01)\end{array}$ & & & & & & & & & & \\
\hline$\Delta \log \mathrm{GL}_{\mathrm{i}, \mathrm{t}-1}$ & & & & & & & & & & & $\begin{array}{l}-0.275 \\
(-1.30)\end{array}$ & $\begin{array}{l}-0.339^{*} \\
(-1.78)\end{array}$ & $\begin{array}{l}-0.293 \\
(-1.38)\end{array}$ & $\begin{array}{l}-0.407 \\
(-1.58)\end{array}$ & $\begin{array}{l}-0.307^{*} \\
(-1.79)\end{array}$ \\
\hline TIER $1_{\mathrm{i}, \mathrm{t}-1}$ & $\begin{array}{c}0.469^{* * * *} \\
(3.61)\end{array}$ & $\begin{array}{c}0.462^{* * * *} \\
(3.52)\end{array}$ & $\begin{array}{c}0.477^{* * * *} \\
(3.66)\end{array}$ & $\begin{array}{c}0.429^{* * * *} \\
(3.00)\end{array}$ & $\begin{array}{c}0.587^{* * * *} \\
(3.09)\end{array}$ & $\begin{array}{l}0.653 \\
(1.48)\end{array}$ & $\begin{array}{l}0.492 \\
(1.19)\end{array}$ & $\begin{array}{c}0.68 \\
(1.53)\end{array}$ & $\begin{array}{l}0.538 \\
(1.16)\end{array}$ & $\begin{array}{l}0.455 \\
(0.84)\end{array}$ & $\begin{array}{l}0.0225 \\
(0.03)\end{array}$ & $\begin{array}{c}0.11 \\
(0.14)\end{array}$ & $\begin{array}{l}0.0372 \\
(0.04)\end{array}$ & $\begin{array}{l}-0.227 \\
(-0.23)\end{array}$ & $\begin{array}{l}0.128 \\
(0.55)\end{array}$ \\
\hline $\operatorname{TIER} 1_{\mathrm{i}, \mathrm{t}-1} * \delta_{\mathrm{t}-1}$ & $\begin{array}{c}0.469^{* * * *} \\
(5.90)\end{array}$ & $\begin{array}{c}0.472^{* * * *} \\
(6.05)\end{array}$ & $\begin{array}{c}0.443^{* * * *} \\
(5.52)\end{array}$ & $\begin{array}{c}0.489^{* * * *} \\
(6.29)\end{array}$ & $\begin{array}{c}0.521^{* * * *} \\
(6.30)\end{array}$ & $\begin{array}{c}0.331^{* * * *} \\
(3.73)\end{array}$ & $\begin{array}{c}0.344^{* * * *} \\
(4.07)\end{array}$ & $\begin{array}{c}0.303^{* * *} \\
(3.36)\end{array}$ & $\begin{array}{c}0.369^{* * * *} \\
(4.44)\end{array}$ & $\begin{array}{c}0.335^{* * * *} \\
(4.17)\end{array}$ & $\begin{array}{l}0.534^{* *} \\
(2.45)\end{array}$ & $\begin{array}{l}0.515^{* *} \\
(2.30)\end{array}$ & $\begin{array}{l}0.497^{* *} \\
(2.25)\end{array}$ & $\begin{array}{l}0.595^{* *} \\
(2.12)\end{array}$ & $\begin{array}{l}0.229^{*} \\
(1.96)\end{array}$ \\
\hline TIER $2_{\mathrm{i}, \mathrm{t}-1}$ & $\begin{array}{l}0.890^{*} \\
(1.85)\end{array}$ & $\begin{array}{l}0.890^{*} \\
(1.87)\end{array}$ & $\begin{array}{l}0.839^{*} \\
(1.72)\end{array}$ & $\begin{array}{l}1.021^{* *} \\
(2.19)\end{array}$ & $\begin{array}{c}1.537^{* *} \\
(2.51)\end{array}$ & $\begin{array}{l}0.505 \\
(0.93)\end{array}$ & $\begin{array}{l}0.382 \\
(0.72)\end{array}$ & $\begin{array}{l}0.447 \\
(0.81)\end{array}$ & $\begin{array}{l}0.829^{*} \\
(1.82)\end{array}$ & $\begin{array}{l}0.84 \\
(1.53)\end{array}$ & $\begin{array}{l}1.156^{* * *} \\
(1.98)\end{array}$ & $\begin{array}{l}1.180^{* * *} \\
(2.09)\end{array}$ & $\begin{array}{l}1.144^{*} \\
(1.95)\end{array}$ & $\begin{array}{l}1.025 \\
(1.45)\end{array}$ & $\begin{array}{l}0.569 \\
(0.80)\end{array}$ \\
\hline TIER $2_{\mathrm{i}, \mathrm{t}-1} * \delta_{\mathrm{t}-1}$ & $\begin{array}{l}0.612 \\
(1.02)\end{array}$ & $\begin{array}{l}0.388 \\
(0.61)\end{array}$ & $\begin{array}{l}0.764 \\
(1.25)\end{array}$ & $\begin{array}{l}0.665 \\
(0.98)\end{array}$ & $\begin{array}{c}0.0725 \\
(0.09)\end{array}$ & $\begin{array}{l}0.537 \\
(0.79)\end{array}$ & $\begin{array}{l}0.359 \\
(0.52)\end{array}$ & $\begin{array}{l}0.743 \\
(1.06)\end{array}$ & $\begin{array}{l}0.382 \\
(0.59)\end{array}$ & $\begin{array}{l}-0.302 \\
(-0.36)\end{array}$ & $\begin{array}{l}0.0129 \\
(0.02)\end{array}$ & $\begin{array}{l}-0.317 \\
(-0.52)\end{array}$ & $\begin{array}{l}0.052 \\
(0.09)\end{array}$ & $\begin{array}{l}-0.0308 \\
(-0.04)\end{array}$ & $\begin{array}{l}0.256 \\
(0.45)\end{array}$ \\
\hline $\mathrm{TCD}_{\mathrm{i}, \mathrm{t}-1}$ & $\begin{array}{c}0.0647 \\
(0.38)\end{array}$ & $\begin{array}{l}0.0495 \\
(0.29)\end{array}$ & $\begin{array}{l}0.0715 \\
(0.42)\end{array}$ & $\begin{array}{c}-0.00235 \\
(-0.01)\end{array}$ & $\begin{array}{c}0.0293 \\
(0.13)\end{array}$ & $\begin{array}{l}0.148 \\
(0.77)\end{array}$ & $\begin{array}{l}0.106 \\
(0.55)\end{array}$ & $\begin{array}{l}0.164 \\
(0.85)\end{array}$ & $\begin{array}{c}0.0294 \\
(0.15)\end{array}$ & $\begin{array}{l}-0.0826 \\
(-0.35)\end{array}$ & $\begin{array}{l}0.0354 \\
(0.15)\end{array}$ & $\begin{array}{c}0.0544 \\
(0.23)\end{array}$ & $\begin{array}{c}0.0552 \\
(0.23)\end{array}$ & $\begin{array}{l}-0.0453 \\
(-0.15)\end{array}$ & $\begin{array}{l}-0.027 \\
(-0.13)\end{array}$ \\
\hline $\mathrm{TCD}_{\mathrm{i}, \mathrm{t}-1} * \delta_{\mathrm{t}-1}$ & $\begin{array}{c}0.261^{* * *} \\
(4.16)\end{array}$ & $\begin{array}{c}0.256^{* * *} \\
(4.09)\end{array}$ & $\begin{array}{c}0.233^{* * * *} \\
(3.47)\end{array}$ & $\begin{array}{c}0.324^{* * * *} \\
(4.19)\end{array}$ & $\begin{array}{l}0.285^{* * *} \\
(3.49)\end{array}$ & $\begin{array}{c}0.254^{* * *} \\
(3.33)\end{array}$ & $\begin{array}{c}0.267^{* * *} \\
(3.59)\end{array}$ & $\begin{array}{c}0.226^{* * *} \\
(2.62)\end{array}$ & $\begin{array}{c}0.277^{* * * *} \\
(2.96)\end{array}$ & $\begin{array}{c}0.327^{* * * *} \\
(2.79)\end{array}$ & $\begin{array}{l}0.199^{* *} \\
(2.12)\end{array}$ & $\begin{array}{l}0.204^{* *} \\
(2.17)\end{array}$ & $\begin{array}{l}0.156 \\
(1.54)\end{array}$ & $\begin{array}{l}0.284^{* *} \\
(2.09)\end{array}$ & $\begin{array}{c}0.256^{* * *} \\
(2.65)\end{array}$ \\
\hline $\mathrm{DEP}_{\mathrm{i}, \mathrm{t}-1}$ & $\begin{array}{l}0.205 \\
(1.01)\end{array}$ & $\begin{array}{l}0.193 \\
(0.95)\end{array}$ & $\begin{array}{l}0.208 \\
(1.03)\end{array}$ & $\begin{array}{l}0.375^{*} \\
(1.68)\end{array}$ & $\begin{array}{l}0.157 \\
(0.69)\end{array}$ & $\begin{array}{l}0.231 \\
(1.05)\end{array}$ & $\begin{array}{l}0.175 \\
(0.81)\end{array}$ & $\begin{array}{l}0.242 \\
(1.10)\end{array}$ & $\begin{array}{l}0.406^{*} \\
(1.68)\end{array}$ & $\begin{array}{l}-0.0153 \\
(-0.05)\end{array}$ & $\begin{array}{c}-0.00369 \\
(-0.01)\end{array}$ & $\begin{array}{l}0.0697 \\
(0.20)\end{array}$ & $\begin{array}{c}0.0166 \\
(0.05)\end{array}$ & $\begin{array}{l}0.233 \\
(0.54)\end{array}$ & $\begin{array}{l}-0.145 \\
(-0.61)\end{array}$ \\
\hline $\mathrm{DEP}_{\mathrm{i}, \mathrm{t}-1} * \delta_{\mathrm{t}-1}$ & $\begin{array}{c}0.0709 \\
(0.56)\end{array}$ & $\begin{array}{c}0.0619 \\
(0.50)\end{array}$ & $\begin{array}{c}0.0666 \\
(0.52)\end{array}$ & $\begin{array}{c}0.00115 \\
(0.01)\end{array}$ & $\begin{array}{c}0.0501 \\
(0.27)\end{array}$ & $\begin{array}{c}0.0956 \\
(0.64)\end{array}$ & $\begin{array}{l}0.117 \\
(0.81)\end{array}$ & $\begin{array}{l}0.0868 \\
(0.57)\end{array}$ & $\begin{array}{c}-0.0202 \\
(-0.12)\end{array}$ & $\begin{array}{l}0.128 \\
(0.55)\end{array}$ & $\begin{array}{c}0.02 \\
(0.12)\end{array}$ & $\begin{array}{c}-0.0313 \\
(-0.19)\end{array}$ & $\begin{array}{l}-0.0119 \\
(-0.07)\end{array}$ & $\begin{array}{c}-0.00382 \\
(-0.02)\end{array}$ & $\begin{array}{c}-0.0164 \\
(-0.06)\end{array}$ \\
\hline $\mathrm{TA}_{\mathrm{i}, t-1}$ & $\begin{array}{c}-0.199^{* * * *} \\
(-6.39)\end{array}$ & $\begin{array}{c}-0.215^{* * *} \\
(-6.55)\end{array}$ & $\begin{array}{c}-0.199^{* * * *} \\
(-6.37)\end{array}$ & $\begin{array}{c}-0.202^{* * * *} \\
(-6.09)\end{array}$ & $\begin{array}{c}-0.156^{* * *} \\
(-3.51)\end{array}$ & $\begin{array}{c}-0.155^{* * *} \\
(-4.02)\end{array}$ & $\begin{array}{c}-0.185^{* * * *} \\
(-4.50)\end{array}$ & $\begin{array}{c}-0.153^{* * * *} \\
(-3.90)\end{array}$ & $\begin{array}{c}-0.156^{* * * *} \\
(-3.76)\end{array}$ & $\begin{array}{c}-0.138^{* * * *} \\
(-3.29)\end{array}$ & $\begin{array}{c}-0.342^{* * * *} \\
(-5.15)\end{array}$ & $\begin{array}{c}-0.339^{* * * * *} \\
(-5.04)\end{array}$ & $\begin{array}{c}-0.337^{* * * *} \\
(-5.00)\end{array}$ & $\begin{array}{c}-0.325^{* * * *} \\
(-4.58)\end{array}$ & $\begin{array}{c}-0.255^{* * * *} \\
(-3.12)\end{array}$ \\
\hline $\mathrm{TA}_{\mathrm{i}, \mathrm{t}-1} * \delta_{\mathrm{t}-1}$ & $\begin{array}{c}0.00492 \\
(0.69)\end{array}$ & $\begin{array}{c}0.00794 \\
(1.08)\end{array}$ & $\begin{array}{c}-0.00233 \\
(-0.28)\end{array}$ & $\begin{array}{c}0.0112^{*} \\
(1.74)\end{array}$ & $\begin{array}{c}0.00408 \\
(0.45)\end{array}$ & $\begin{array}{c}-0.00237 \\
(-0.27)\end{array}$ & $\begin{array}{c}0.00161 \\
(0.18)\end{array}$ & $\begin{array}{c}-0.00937 \\
(-0.90)\end{array}$ & $\begin{array}{c}0.00532 \\
(0.74)\end{array}$ & $\begin{array}{c}0.00101 \\
(0.09)\end{array}$ & $\begin{array}{c}0.0193^{*} \\
(1.89)\end{array}$ & $\begin{array}{c}0.0242^{* * *} \\
(2.41)\end{array}$ & $\begin{array}{l}0.0134 \\
(1.21)\end{array}$ & $\begin{array}{c}0.0311^{* * * *} \\
(2.82)\end{array}$ & $\begin{array}{l}-0.0109 \\
(-1.15)\end{array}$ \\
\hline $\operatorname{LLP}_{\mathrm{i}, \mathrm{t}-1}$ & $\begin{array}{l}-1.132 \\
(-1.41)\end{array}$ & $\begin{array}{l}-1.134 \\
(-1.43)\end{array}$ & $\begin{array}{l}-1.133 \\
(-1.41)\end{array}$ & $\begin{array}{l}-1.233 \\
(-1.52)\end{array}$ & $\begin{array}{l}-0.407 \\
(-0.49)\end{array}$ & $\begin{array}{c}-0.4 \\
(-0.40)\end{array}$ & $\begin{array}{l}-0.598 \\
(-0.63)\end{array}$ & $\begin{array}{l}-0.397 \\
(-0.40)\end{array}$ & $\begin{array}{l}-0.618 \\
(-0.59)\end{array}$ & $\begin{array}{c}0.15 \\
(0.16)\end{array}$ & $\begin{array}{l}-0.919 \\
(-0.69)\end{array}$ & $\begin{array}{l}-0.757 \\
(-0.59)\end{array}$ & $\begin{array}{l}-0.889 \\
(-0.67)\end{array}$ & $\begin{array}{l}-1.126 \\
(-0.75)\end{array}$ & $\begin{array}{l}-0.412 \\
(-1.21)\end{array}$ \\
\hline $\mathrm{FA}_{\mathrm{i}, \mathrm{t}-1}$ & $\begin{array}{l}0.608 \\
(0.88)\end{array}$ & $\begin{array}{l}0.596 \\
(0.89)\end{array}$ & $\begin{array}{l}0.594 \\
(0.85)\end{array}$ & $\begin{array}{l}0.828 \\
(1.14)\end{array}$ & $\begin{array}{c}2.149^{* *} \\
(2.16)\end{array}$ & $\begin{array}{l}1.016 \\
(1.21)\end{array}$ & $\begin{array}{l}1.146 \\
(1.41)\end{array}$ & $\begin{array}{l}0.979 \\
(1.16)\end{array}$ & $\begin{array}{l}1.281 \\
(1.48)\end{array}$ & $\begin{array}{l}2.261^{* *} \\
(2.42)\end{array}$ & $\begin{array}{l}0.333 \\
(0.36)\end{array}$ & $\begin{array}{l}0.135 \\
(0.15)\end{array}$ & $\begin{array}{l}0.305 \\
(0.33)\end{array}$ & $\begin{array}{l}0.512 \\
(0.53)\end{array}$ & $\begin{array}{l}1.833^{*} \\
(1.66)\end{array}$ \\
\hline $\mathrm{ROA}_{\mathrm{i}, \mathrm{t}-1}$ & $\begin{array}{l}0.399 \\
(0.60)\end{array}$ & $\begin{array}{l}0.411 \\
(0.63)\end{array}$ & $\begin{array}{l}0.403 \\
(0.60)\end{array}$ & $\begin{array}{l}0.397 \\
(0.58)\end{array}$ & $\begin{array}{l}0.707 \\
(0.93)\end{array}$ & $\begin{array}{l}0.594 \\
(0.79)\end{array}$ & $\begin{array}{c}0.22 \\
(0.31)\end{array}$ & $\begin{array}{l}0.592 \\
(0.79)\end{array}$ & $\begin{array}{l}0.536 \\
(0.68)\end{array}$ & $\begin{array}{l}0.577 \\
(0.87)\end{array}$ & $\begin{array}{l}0.104 \\
(0.17)\end{array}$ & $\begin{array}{c}0.22 \\
(0.37)\end{array}$ & $\begin{array}{l}0.129 \\
(0.21)\end{array}$ & $\begin{array}{l}0.187 \\
(0.27)\end{array}$ & $\begin{array}{l}0.935 \\
(1.13)\end{array}$ \\
\hline$\delta_{t-1}$ & $\begin{array}{c}-0.290^{* * *} \\
(-2.29)\end{array}$ & $\begin{array}{c}-0.278^{* *} \\
(-2.14)\end{array}$ & $\begin{array}{l}-0.169 \\
(-1.15)\end{array}$ & $\begin{array}{c}-0.363^{* * * *} \\
(-2.73)\end{array}$ & $\begin{array}{l}-0.235 \\
(-1.36)\end{array}$ & $\begin{array}{l}-0.161 \\
(-1.00)\end{array}$ & $\begin{array}{l}-0.158 \\
(-1.00)\end{array}$ & $\begin{array}{c}-0.0466 \\
(-0.25)\end{array}$ & $\begin{array}{l}-0.259^{*} \\
(-1.78)\end{array}$ & $\begin{array}{l}-0.192 \\
(-0.83)\end{array}$ & $\begin{array}{l}-0.501^{* * *} \\
(-2.37)\end{array}$ & $\begin{array}{l}-0.503^{* *} \\
(-2.32)\end{array}$ & $\begin{array}{l}-0.389^{*} \\
(-1.69)\end{array}$ & $\begin{array}{c}-0.697^{* * *} \\
(-2.95)\end{array}$ & $\begin{array}{c}0.0268 \\
(0.14)\end{array}$ \\
\hline Macroeconomic variable & & & & & & & & & & & & & & & \\
\hline GDP growth $_{\mathrm{i}, \mathrm{t}}$ & $\begin{array}{c}0.923^{* * * 9} \\
(5.86)\end{array}$ & $\begin{array}{c}1.014^{* * * * 7} \\
(6.34)\end{array}$ & $\begin{array}{c}0.961^{N * * *} \\
(5.96)\end{array}$ & $\begin{array}{c}0.950^{2 m+4} \\
(5.65)\end{array}$ & $\begin{array}{c}0.697^{* 2 * 4} \\
(3.49)\end{array}$ & $\begin{array}{c}0.907^{* * * 4} \\
(5.25)\end{array}$ & $\begin{array}{c}1.069^{2 * 2 \times 4} \\
(5.92)\end{array}$ & $\begin{array}{c}0.946^{\text {श*F }} \\
(5.26)\end{array}$ & $\begin{array}{c}0.880^{n+m} \\
(4.66)\end{array}$ & $\begin{array}{l}0.804^{2} \\
(3.96)\end{array}$ & $\begin{array}{c}0.872^{m+n} \\
(4.33)\end{array}$ & $\begin{array}{c}0.885^{* * 27} \\
(4.35)\end{array}$ & $\begin{array}{c}0.890^{m+n} \\
(4.35)\end{array}$ & $\begin{array}{c}0.994^{\text {*N*FF }} \\
(4.55)\end{array}$ & $\begin{array}{c}0.617^{* 2 \times n} \\
(3.29)\end{array}$ \\
\hline Interest rate $\mathrm{i}_{\mathrm{i}, \mathrm{t}}$ & $\begin{array}{c}-0.559^{* * *} \\
(-3.66)\end{array}$ & $\begin{array}{c}-0.593^{* * *} \\
(-3.59)\end{array}$ & $\begin{array}{c}-0.557^{* * *} \\
(-3.64)\end{array}$ & $\begin{array}{c}-0.572^{* * * *} \\
(-3.57)\end{array}$ & $\begin{array}{l}-0.384^{* *} \\
(-2.33)\end{array}$ & $\begin{array}{l}-0.396^{* *} \\
(-2.37)\end{array}$ & $\begin{array}{c}-0.543^{* * * *} \\
(-3.08)\end{array}$ & $\begin{array}{l}-0.391^{* *} \\
(-2.31)\end{array}$ & $\begin{array}{c}-0.376^{* *} \\
(-2.14)\end{array}$ & $\begin{array}{l}-0.146 \\
(-0.92)\end{array}$ & $\begin{array}{l}-0.305^{* *} \\
(-2.02)\end{array}$ & $\begin{array}{l}-0.329^{* *} \\
(-2.09)\end{array}$ & $\begin{array}{l}-0.303^{* * *} \\
(-1.99)\end{array}$ & $\begin{array}{l}-0.256 \\
(-1.48)\end{array}$ & $\begin{array}{l}-0.164 \\
(-1.16)\end{array}$ \\
\hline
\end{tabular}


Table B.3. Estimation Result of Commercial Banks

\begin{tabular}{|c|c|c|c|c|c|c|c|c|c|c|c|c|c|c|c|}
\hline \multirow{2}{*}{\multicolumn{16}{|c|}{ Industry structure variables }} \\
\hline & & & & & & & & & & & & & & & \\
\hline \multirow[t]{2}{*}{$\mathrm{HHI}_{\mathrm{i}, \mathrm{t}-1}$} & & $-0.888^{*}$ & & & & & $-1.827^{* * *}$ & & & & & -0.715 & & & \\
\hline & & $(-1.87)$ & & & & & $(-2.66)$ & & & & & $(-1.12)$ & & & \\
\hline \multirow{2}{*}{$\mathrm{HHI}_{\mathrm{i}, \mathrm{t}-1} * \delta_{\mathrm{t}-1}$} & & 0.276 & & & & & $0.475^{*}$ & & & & & 0.183 & & & \\
\hline & & (1.23) & & & & & $(1.70)$ & & & & & $(0.77)$ & & & \\
\hline \multirow[t]{2}{*}{ COMPTIER $1_{\mathrm{i}, \mathrm{t}-1}$} & & $0.488^{* * * *}$ & & & & & $0.676^{* * * *}$ & & & & & $0.686^{* * * *}$ & & & \\
\hline & & $(3.04)$ & & & & & $(4.28)$ & & & & & $(3.20)$ & & & \\
\hline \multirow[t]{2}{*}{ COMPTIER $1_{\mathrm{i},-1-1} * \delta_{\mathrm{t}-1}$} & & $-0.637^{* * *}$ & & & & & $-0.876^{* * * *}$ & & & & & $-0.755^{* * *}$ & & & \\
\hline & & $(-3.45)$ & & & & & $(-4.60)$ & & & & & $(-3.75)$ & & & \\
\hline \multicolumn{16}{|l|}{ Bank type variables } \\
\hline \multirow[t]{2}{*}{ Government dummy $* \delta_{t-1}$} & & & $0.0514^{m, n}$ & & & & & $0.0468^{*}$ & & & & & $0.0512^{*}$ & & \\
\hline & & & $(2.38)$ & & & & & $(1.72)$ & & & & & $(1.86)$ & & \\
\hline \multirow{2}{*}{ Foreign dummy $* \delta_{t-1}$} & & & $-0.0913^{*}$ & & & & & $-0.126^{* *}$ & & & & & -0.0324 & & \\
\hline & & & $(-1.78)$ & & & & & $(-2.27)$ & & & & & $(-0.76)$ & & \\
\hline \multirow[t]{2}{*}{ Subsidiary dummy $* \delta_{t-1}$} & & & 0.0147 & & & & & $0.0607^{*}$ & & & & & 0.0138 & & \\
\hline & & & $(0.53)$ & & & & & $(1.84)$ & & & & & $(0.38)$ & & \\
\hline \multicolumn{16}{|l|}{ Regulation variables } \\
\hline \multirow{2}{*}{\multicolumn{2}{|c|}{ Overall capital stringency $* \delta_{t-1}$}} & & & $-0.0226^{* *}$ & & & & & -0.0127 & & & & & -0.0193 & \\
\hline & & & & $(-2.33)$ & & & & & $(-1.11)$ & & & & & $(-1.10)$ & \\
\hline \multirow{2}{*}{ Deposit insurance $* \delta_{\mathrm{t}-1}$} & & & & $0.0454^{*}$ & & & & & 0.0272 & & & & & 0.0137 & \\
\hline & & & & $(1.92)$ & & & & & $(1.05)$ & & & & & $(0.51)$ & \\
\hline Bail-out probability $* \delta_{\mathrm{t}-1}$ & & & & & $\begin{array}{c}-0.0763^{* * *} \\
(-2.05)\end{array}$ & & & & & $\begin{array}{r}-0.0788^{*} \\
(-1.93)\end{array}$ & & & & & $\begin{array}{c}-0.0533 \\
(-1.07)\end{array}$ \\
\hline$N$ & 3849 & 3848 & 3849 & 3256 & 1822 & 2796 & 2796 & 2796 & 2315 & 1461 & 2382 & 2382 & 2382 & 2024 & 1236 \\
\hline Adjusted-R2 & 0.189 & 0.201 & 0.191 & 0.218 & 0.269 & & & & & & & & & & \\
\hline Hansen J Statistic ( $p$-value) & & & & & & $5.525(0.06)$ & $8.351(0.04)$ & $5.969(0.05)$ & $3.179(0.2)$ & $2.672(0.3)$ & $20.73(0.05)$ & $29.27(0.004)$ & $21.25(0.06))$ & $17.13(0.1)$ & $19.45(0.1)$ \\
\hline Estimation Method & FE & $\mathrm{FE}$ & $\mathrm{FE}$ & $\mathrm{FE}$ & $\mathrm{FE}$ & IV & IV & IV & IV & IV & GMM & GMM & GMM & GMM & GMM \\
\hline
\end{tabular}

Note. The dependent variable is $\Delta \operatorname{logGL}, \mathrm{it}$. Estimation methods are FE, IV and GMM. T-values are reported in parentheses. Superscripts ***, $* *, *$ indicate significance levels of $1 \%, 5 \%$, and $10 \%$ respectively. 


\section{Appendix C: Chapter 3}

Table C.1. Summary of Empirical Analysis on Clearing and Settlement Institutions (Chronologically)

\begin{tabular}{|c|c|c|c|c|c|}
\hline Author(year) & Sample & Research Question & Method & Indicator & Findings \\
\hline $\begin{array}{l}\text { Bauer and } \\
\text { Ferrier (1996) }\end{array}$ & $\begin{array}{l}\text { 1990-1994, } \\
\text { The Federal Reserve } \\
\text { Payments system }\end{array}$ & $\begin{array}{l}\text { Scale Economies, Cost } \\
\text { Efficiencies, and } \\
\text { Technological Change in } \\
\text { Federal Reserve Payments } \\
\text { Processing }\end{array}$ & $\begin{array}{l}\text { Cross-section } \\
\text { Analysis }\end{array}$ & $\begin{array}{l}\text { Technical } \\
\text { Changes, } \\
\text { Input Prices, } \\
\text { Scale, } \\
\text { Cost } \\
\text { Efficiency }\end{array}$ & $\begin{array}{l}\text { Significant scale economies are found in all three ACH processing } \\
\text { sites and Cost Efficiencies can be improved. }\end{array}$ \\
\hline $\begin{array}{l}\text { Hancock, } \\
\text { Humphrey, and } \\
\text { Wilcox (1999) }\end{array}$ & $\begin{array}{l}\text { 1979-1996, Fedwire } \\
\text { Payment Processing }\end{array}$ & $\begin{array}{l}\text { Potential Effects of } \\
\text { Consolidation on Fedwire } \\
\text { Operations }\end{array}$ & $\begin{array}{l}\text { Cross-section } \\
\text { Analysis }\end{array}$ & $\begin{array}{l}\text { Technical } \\
\text { Changes, } \\
\text { Scale, } \\
\text { Cost Effects }\end{array}$ & $\begin{array}{l}\text { The Fedwire funds transfer operation exhibited large scale } \\
\text { economies but little technical advance beyond that already } \\
\text { embodied in the technology-adjusted input prices of data } \\
\text { processing and telecommunication inputs. } \\
\text { The consolidation of Fedwire into fewer offices contributed } \\
\text { around one-fourth of the overall reduction in Fedwire average } \\
\text { cost. }\end{array}$ \\
\hline $\begin{array}{l}\text { Hasan and } \\
\text { Malkamäki } \\
(2001)\end{array}$ & $\begin{array}{l}\text { 1989-1998, } \\
38 \text { Exchanges in } 32 \\
\text { Countries }\end{array}$ & $\begin{array}{l}\text { Investigates the Existence and } \\
\text { Extent of Economies of Scale } \\
\text { and Scope Among the Stock } \\
\text { Exchanges }\end{array}$ & $\begin{array}{l}\text { Cross-section } \\
\text { Analysis }\end{array}$ & $\begin{array}{l}\text { Economies of } \\
\text { Scale, } \\
\text { Economies of } \\
\text { Scope, }\end{array}$ & $\begin{array}{l}\text { The largest exchanges show an increasing trend of cost } \\
\text { effectiveness. } \\
\text { The exchanges in North America and Europe report substantially } \\
\text { higher economies of scale relative to the exchanges in the } \\
\text { Asia-Pacific regions. }\end{array}$ \\
\hline $\begin{array}{l}\text { Lannoo and } \\
\text { Levin (2002) }\end{array}$ & $\begin{array}{l}\text { Securities Settlement } \\
\text { Systems in EU and } \\
\text { U.S. }\end{array}$ & $\begin{array}{l}\text { Verify Whether the Costs for } \\
\text { Cross-border Securities } \\
\text { Settlement were Indeed as } \\
\text { High as Often Assumed. }\end{array}$ & $\begin{array}{l}\text { Panel } \\
\text { Analysis }\end{array}$ & $\begin{array}{l}\text { Cost/Income } \\
\text { Structure }\end{array}$ & $\begin{array}{l}\text { The operating costs of securities settlement are higher in the EU } \\
\text { than in the U.S. } \\
\text { Cross-border settlement is much more costly than its domestic } \\
\text { counterpart. }\end{array}$ \\
\hline $\begin{array}{l}\text { Hasan, } \\
\text { Malkamäki, and } \\
\text { Schmiedel } \\
\text { (2003) }\end{array}$ & $\begin{array}{l}\text { 1989-1998, } \\
49 \text { Stock Exchanges }\end{array}$ & $\begin{array}{l}\text { The Productivity of Stock } \\
\text { Exchanges over Time and } \\
\text { Across Different Types and } \\
\text { Groups of Exchanges }\end{array}$ & $\begin{array}{l}\text { Cross-section } \\
\text { Analysis }\end{array}$ & $\begin{array}{l}\text { Revenue and } \\
\text { Cost } \\
\text { Efficiency, } \\
\text { Technological } \\
\text { Development }\end{array}$ & $\begin{array}{l}\text { The findings indicate significant variability in productivity- } \\
\text { revenue and cost efficiency - across these exchanges. } \\
\text { North American exchanges are found to be most cost and revenue } \\
\text { efficient. } \\
\text { European exchanges have improved the most, in respect of cost } \\
\text { efficiency, while exchanges in South America and Asia-Pacific } \\
\text { are found to be lagging as regards both cost and revenue } \\
\text { estimations. } \\
\text { Investment in technology-related developments effectively } \\
\text { influenced cost and revenue efficiency. } \\
\text { Organizational structure and market competition are found to be } \\
\text { significantly associated with both cost and revenue efficiency for }\end{array}$ \\
\hline
\end{tabular}


Table C.1. Summary of Empirical Analysis on Clearing and Settlement Institutions (Chronologically)

\begin{tabular}{|c|c|c|c|c|c|}
\hline Author(year) & Sample & Research Question & Method & Indicator & Findings \\
\hline & & & & & $\begin{array}{l}\text { the exchanges studied, whereas market size and quality are related } \\
\text { only to revenue efficiency. }\end{array}$ \\
\hline $\begin{array}{l}\text { Schmiedel } \\
(2001)\end{array}$ & $\begin{array}{l}1985-1999, \\
\text { Exchanges in } \\
\text { European }\end{array}$ & $\begin{array}{l}\text { The Existence and Extent of } \\
\text { Technical Inefficiencies of } \\
\text { Financial } \\
\text { Exchanges in Europe. }\end{array}$ & $\begin{array}{l}\text { Cross-section } \\
\text { Analysis }\end{array}$ & $\begin{array}{l}\text { Technical } \\
\text { Inefficiencies } \\
\text { Scores }\end{array}$ & $\begin{array}{l}\text { Size of exchange; market concentration and quality; structural } \\
\text { reorganizations of exchange governance; diversification in trading } \\
\text { service activities; and adoption of automated trading systems } \\
\text { significantly influence the efficient provision of trading services in } \\
\text { Europe. } \\
\text { European exchanges notably improved their ability to efficiently } \\
\text { manage their production and input resources }\end{array}$ \\
\hline NERA (2004) & $\begin{array}{l}\text { Clearing and } \\
\text { Settlement Systems } \\
\text { in EU and U.S. }\end{array}$ & $\begin{array}{l}\text { Investigates the Direct Costs } \\
\text { of Clearing and Settling an } \\
\text { Equity } \\
\text { Transaction in Europe and in } \\
\text { the U.S. }\end{array}$ & $\begin{array}{l}\text { Panel } \\
\text { Analysis }\end{array}$ & $\begin{array}{l}\text { Cost } \\
\text { Structure }\end{array}$ & $\begin{array}{l}\text { There is a significant gap between the costs of clearing and } \\
\text { settlement in the U.S. and Europe. } \\
\text { Cost differentials between the U.S. and Europe are higher for } \\
\text { non-domestic than for domestic transactions. } \\
\text { Costs differ very significantly within Europe. }\end{array}$ \\
\hline $\begin{array}{l}\text { Rosati and } \\
\text { Secola (2006) }\end{array}$ & $\begin{array}{l}\text { 1999-2002, Payment } \\
\text { System TARGET } \\
\text { and EURO1 }\end{array}$ & $\begin{array}{l}\text { Distribution of the TARGET } \\
\text { Cross-border Interbank } \\
\text { Payment Flows }\end{array}$ & $\begin{array}{l}\text { Time-series } \\
\text { analysis }\end{array}$ & $\begin{array}{l}\text { Trading } \\
\text { Volumes, } \\
\text { Location, }\end{array}$ & $\begin{array}{l}\text { That bilateral payment flows reflects an organization of interbank } \\
\text { trading between countries in which the size of the banking sector, } \\
\text { geographic proximity and cultural similarities play a significant } \\
\text { role. } \\
\text { The payment traffic in TARGET is strongly affected by technical } \\
\text { market deadlines. }\end{array}$ \\
\hline $\begin{array}{l}\text { Schmiedel et al. } \\
(2006)\end{array}$ & $\begin{array}{l}1993-2000, \\
16 \text { Settlement } \\
\text { Institutions }\end{array}$ & $\begin{array}{l}\text { Investigates the Existence and } \\
\text { Extent of Economies of Scale } \\
\text { in Depository and Settlement } \\
\text { Systems }\end{array}$ & $\begin{array}{l}\text { Cross-section } \\
\text { Analysis }\end{array}$ & $\begin{array}{l}\text { Economies of } \\
\text { Scale, } \\
\text { Technological } \\
\text { Development }\end{array}$ & $\begin{array}{l}\text { The degree of economies differs by size of settlement institution } \\
\text { and region. } \\
\text { While smaller settlement service providers reveal a high potential } \\
\text { of economies for scale, larger institutions show an increasing } \\
\text { trend toward cost effectiveness. } \\
\text { Clearing and settlement systems in countries in Europe and Asia } \\
\text { report substantially larger economies of scale than those of the } \\
\text { U.S. system. } \\
\text { European cross-border settlement seems to be more cost intensive } \\
\text { than that on a domestic level, reflecting chiefly complexities of } \\
\text { EU international securities settlement systems and differences in } \\
\text { the scope of international settlement service providers. } \\
\text { Investments in implementing new systems and upgrades of } \\
\text { settlement technology continuously improved cost effectiveness } \\
\text { over the sample period }\end{array}$ \\
\hline Milne (2007b) & $\begin{array}{l}\text { 1997-2000, } \\
16 \text { Securities } \\
\text { Settlement }\end{array}$ & & $\begin{array}{l}\text { Cross-section } \\
\text { Analysis }\end{array}$ & & $\begin{array}{l}\text { Identified substantial economies of both scale and scope and } \\
\text { important interactions with trading platforms. }\end{array}$ \\
\hline
\end{tabular}


Table C.1. Summary of Empirical Analysis on Clearing and Settlement Institutions (Chronologically)

\begin{tabular}{|c|c|c|c|c|c|}
\hline Author(year) & Sample & Research Question & Method & Indicator & Findings \\
\hline & $\begin{array}{l}\text { Institutions in } \\
\text { Europe and U.S. }\end{array}$ & & & & \\
\hline $\begin{array}{l}\text { Van Cayseele } \\
\text { and Wuyts } \\
(2007)\end{array}$ & $\begin{array}{l}\text { 1997-2004, } \\
10 \text { Settlement } \\
\text { Institutions in } \\
\text { Europe }\end{array}$ & $\begin{array}{l}\text { Cost Efficiency in the } \\
\text { European Securities } \\
\text { Settlement and Depository } \\
\text { Industry }\end{array}$ & $\begin{array}{l}\text { Cross-section } \\
\text { Analysis }\end{array}$ & $\begin{array}{l}\text { Cost } \\
\text { Efficiency, } \\
\text { Economies of } \\
\text { Scale, } \\
\text { Economies of } \\
\text { Scope }\end{array}$ & $\begin{array}{l}\text { The findings indicate that especially the smaller institutions still } \\
\text { can realize many scale economies. This constitutes a rationale for } \\
\text { further consolidation. } \\
\text { Separating settlement from issuance services, and hosting them in } \\
\text { different entities will entail efficiency losses and cost increases. }\end{array}$ \\
\hline $\begin{array}{l}\text { Beijnen and Bolt } \\
\text { (2009) }\end{array}$ & $\begin{array}{l}1990-2005,8 \\
\text { Institutions in } \\
\text { Europe }\end{array}$ & $\begin{array}{l}\text { The Existence and Extent of } \\
\text { Economies of Scale in the } \\
\text { European Payment Processing } \\
\text { Industry. }\end{array}$ & $\begin{array}{l}\text { Cross-section } \\
\text { Analysis }\end{array}$ & $\begin{array}{l}\text { Economies of } \\
\text { Scale, }\end{array}$ & $\begin{array}{l}\text { The existence of significant economies of scale. } \\
\text { Ownership structure is an important factor to explain cost } \\
\text { differences across European processing centers }\end{array}$ \\
\hline Nielsson (2009) & $\begin{array}{l}2000, \\
\text { Merger Events in } \\
\text { Euronext }\end{array}$ & $\begin{array}{l}\text { How Exchange Consolidation } \\
\text { has Affected Stock Liquidity } \\
\text { and How the Effect Varies } \\
\text { with Firm Type }\end{array}$ & $\begin{array}{l}\text { Panel } \\
\text { Analysis }\end{array}$ & $\begin{array}{l}\text { Liquidity, } \\
\text { Merger }\end{array}$ & $\begin{array}{l}\text { Asymmetric liquidity gains from the stock exchange merger, } \\
\text { where the positive effects are concentrated among big firms and } \\
\text { firms with foreign sales. } \\
\text { The merger is associated with an increase in Euronext's market } \\
\text { share, where the increase is drawn from the } \\
\text { London Stock Exchange. }\end{array}$ \\
\hline $\begin{array}{l}\text { Hasan, } \\
\text { Schmiedel, and } \\
\text { Song }(2012)\end{array}$ & $\begin{array}{l}2000-2008 \\
15 \text { Public Stock } \\
\text { Exchanges in } 12 \\
\text { Countries }\end{array}$ & $\begin{array}{l}\text { Investigate Short-run Share } \\
\text { Price of Stock Exchanges } \\
\text { Responses to the Formation } \\
\text { of M\&As and Alliances }\end{array}$ & $\begin{array}{l}\text { Cross-section } \\
\text { Analysis }\end{array}$ & $\begin{array}{l}\text { Merger, } \\
\text { Cumulative } \\
\text { Return, } \\
\text { Technological } \\
\text { Integration }\end{array}$ & $\begin{array}{l}\text { The average stock price responses for M\&As and alliances are } \\
\text { positive. M\&As create more value than alliances. } \\
\text { For alliances, joint ventures generate more value than non-equity } \\
\text { alliances. More value accrues when the integration is horizontal } \\
\text { than when it is vertical. } \\
\text { Cross-border integration creates more value than domestic } \\
\text { integration. } \\
\text { Finally, the findings indicate that when the partnering exchange is } \\
\text { located in a country with better shareholder protection, accounting } \\
\text { standards, and capital market development, more shareholder } \\
\text { value accrues to our sample exchange. These patterns are } \\
\text { consistent when we examine the exchanges' long-run } \\
\text { performance. }\end{array}$ \\
\hline
\end{tabular}


Table C.2. Empirical Evidence on the Use of Panzar-Rosse Model and Lerner Index (Chronologically)

\begin{tabular}{|c|c|c|c|c|c|}
\hline Author (Year) & Sample & $\begin{array}{l}\text { Financial } \\
\text { Indicators }\end{array}$ & $\begin{array}{l}\text { Dependent } \\
\text { Variable }\end{array}$ & Scaling & Key Findings \\
\hline Shaffer (1982) & $\begin{array}{l}\text { 1979, } \\
\text { New Year banks }\end{array}$ & $\begin{array}{l}\text { Panzar-Rosse } \\
H \text {-Statistic }\end{array}$ & $\ln$ II & $\ln \mathrm{TA}$ & Monopolistic Competition \\
\hline $\begin{array}{l}\text { Nathan and } \\
\text { Neave (1989) }\end{array}$ & $\begin{array}{l}\text { 1982-1984, } \\
\text { Canadian banks }\end{array}$ & $\begin{array}{l}\text { Panzar-Rosse } \\
H \text {-Statistic }\end{array}$ & $\ln \mathrm{TI}$ & $\ln \mathrm{TA}$ & 1982: perfect competition; 1983 and 1984: monopolistic Competition \\
\hline Shaffer (1993) & $\begin{array}{l}\text { 1965-1989, } \\
\text { Canadian Banks }\end{array}$ & Lerner Index & & & Perfect competition \\
\hline $\begin{array}{l}\text { Molyneux et al. } \\
\text { (1994) }\end{array}$ & $\begin{array}{l}\text { 1986-1989, France, } \\
\text { Germany, Italy, Spain and } \\
\text { UK }\end{array}$ & $\begin{array}{l}\text { Panzar-Rosse } \\
H \text {-Statistic }\end{array}$ & $\ln$ II & $\ln \mathrm{TA}$ & $\begin{array}{l}\text { Monopoly: Italy: } \\
\text { Monopolistic Competition: France, Germany, Spain, UK }\end{array}$ \\
\hline $\begin{array}{l}\text { Vesala and } \\
\text { Pankki (1995) }\end{array}$ & 1985-1992, Finland & $\begin{array}{l}\text { Panzar-Rosse } \\
H \text {-Statistic }\end{array}$ & $\ln$ II & $\ln \mathrm{TA}$ & $\begin{array}{l}\text { Monopoly: 1989-1990; } \\
\text { Monopolistic Competition: 1985-1988.1991-1002 }\end{array}$ \\
\hline $\begin{array}{l}\text { Molyneux et al. } \\
\text { (1996) }\end{array}$ & 1986-1988, Japan & $\begin{array}{l}\text { Panzar-Rosse } \\
H \text {-Statistic }\end{array}$ & $\ln$ II & $\begin{array}{l}\ln \mathrm{TA} \\
\ln \mathrm{TD}\end{array}$ & $\begin{array}{l}\text { Monopoly: 1986; } \\
\text { Monopolistic Competition: 1987-1988 }\end{array}$ \\
\hline Coccorese (1998) & 1988-1996, Italy & $\begin{array}{l}\text { Panzar-Rosse } \\
H \text {-Statistic }\end{array}$ & $\ln \mathrm{TI}$ & $\begin{array}{l}\ln \mathrm{TA} \\
\ln \mathrm{TD}\end{array}$ & Monopolistic Competition \\
\hline Shaffer (1999) & 1984-1993, U.S. & Lerner Index & & & Monopolistic Competition \\
\hline $\begin{array}{l}\text { Hondroyiannis et } \\
\text { al. (1999) }\end{array}$ & 1993-1995, Greece & $\begin{array}{l}\text { Panzar-Rosse } \\
H \text {-Statistic }\end{array}$ & $\ln (\mathrm{TI} / \mathrm{TA})$ & $\ln \mathrm{TA}$ & Monopolistic Competition \\
\hline $\begin{array}{l}\text { Bikker and } \\
\text { Groeneveld } \\
(2000)\end{array}$ & $\begin{array}{l}\text { 1989-1996, } \\
15 \text { EU countries }\end{array}$ & $\begin{array}{l}\text { Panzar-Rosse } \\
H \text {-Statistic }\end{array}$ & $\ln (\mathrm{II} / \mathrm{TA})$ & $\ln \mathrm{TA}$ & Monopolistic Competition \\
\hline $\begin{array}{l}\text { De Bandt and } \\
\text { Davis (2000) }\end{array}$ & $\begin{array}{l}\text { 1992-1996, France, } \\
\text { Germany and Italy }\end{array}$ & $\begin{array}{l}\text { Panzar-Rosse } \\
H \text {-Statistic }\end{array}$ & $\begin{array}{l}\ln \mathrm{II} ; \\
\ln \mathrm{TI}\end{array}$ & $\ln \mathrm{EQ}$ & $\begin{array}{l}\text { Monopolistic Competition: Large Banks; Small Banks in Italy; } \\
\text { Monopoly: Small Banks in France and Germany. }\end{array}$ \\
\hline $\begin{array}{l}\text { Levine et al. } \\
(2000)\end{array}$ & 1960-1995, 74 countries, & $\begin{array}{l}\text { Panzar-Rosse } \\
H \text {-Statistic }\end{array}$ & GDP growth & & $\begin{array}{l}\text { Legal and accounting reforms that strengthen creditor rights, contract } \\
\text { enforcement, and accounting practices can boost financial } \\
\text { development and accelerate economic growth. }\end{array}$ \\
\hline Hempell (2002) & 1993-1998, Germany & $\begin{array}{l}\text { Panzar-Rosse } \\
H \text {-Statistic }\end{array}$ & $\ln (\mathrm{TI} / \mathrm{TA})$ & & Monopolistic Competition \\
\hline $\begin{array}{l}\text { Bikker and Haaf } \\
(2002)\end{array}$ & $\begin{array}{l}\text { 1988-1998, } \\
23 \text { OECD Countries }\end{array}$ & $\begin{array}{l}\text { Panzar-Rosse } \\
H \text {-Statistic }\end{array}$ & $\ln (\mathrm{II} / \mathrm{TA})$ & $\ln \mathrm{TA}$ & Monopolistic Competition \\
\hline Shaffer (2002) & $\begin{array}{l}\text { 1985-2000, } \\
\text { U.S. banks }\end{array}$ & $\begin{array}{l}\text { Panzar-Rosse } \\
H \text {-Statistic }\end{array}$ & $\ln \mathrm{TI}$ & $\ln \mathrm{TA}$ & Monopolistic Competition \\
\hline $\begin{array}{l}\text { Murjan and Ruza } \\
(2002)\end{array}$ & $\begin{array}{l}\text { 1993-1997, } \\
\text { Arab Middle East }\end{array}$ & $\begin{array}{l}\text { Panzar-Rosse } \\
H \text {-Statistic }\end{array}$ & $\ln$ II & $\ln \mathrm{TA}$ & Monopolistic Competition \\
\hline
\end{tabular}


Table C.2. Empirical Evidence on the Use of Panzar-Rosse Model and Lerner Index (Chronologically)

\begin{tabular}{|c|c|c|c|c|c|}
\hline Author (Year) & Sample & $\begin{array}{l}\text { Financial } \\
\text { Indicators }\end{array}$ & $\begin{array}{l}\text { Dependent } \\
\text { Variable } \\
\end{array}$ & Scaling & Key Findings \\
\hline $\begin{array}{l}\text { Collender and } \\
\text { Shaffer (2003) }\end{array}$ & & $\begin{array}{l}\text { Panzar-Rosse } \\
\mathrm{H} \text {-Statistic } \\
\end{array}$ & & & \\
\hline $\begin{array}{l}\text { Yeyati and Micco } \\
(2003)\end{array}$ & $\begin{array}{l}\text { 1993-2002, } \\
\text { Latin America }\end{array}$ & $\begin{array}{l}\text { Panzar-Rosse } \\
H \text {-Statistic }\end{array}$ & $\ln (\mathrm{TI} / \mathrm{TA})$ & $\ln \mathrm{TA}$ & $\begin{array}{l}\text { Monopolistic Competition: Argentina, Brazil, Colombia, Costa Rica, } \\
\text { Peru, EI Salvador; } \\
\text { Perfect Competition: Chile. }\end{array}$ \\
\hline Coccorese (2004) & 1997-199, Italy & $\begin{array}{l}\text { Panzar-Rosse } \\
\mathrm{H} \text {-Statistic }\end{array}$ & $\begin{array}{l}\ln \mathrm{II} \\
\ln \mathrm{TI}\end{array}$ & $\ln \mathrm{TA}$ & Monopolistic Competition \\
\hline $\begin{array}{l}\text { Claessens and } \\
\text { Leaven (2004) }\end{array}$ & $\begin{array}{l}\text { 1994-2001, } \\
50 \text { Countries, }\end{array}$ & $\begin{array}{l}\text { Panzar-Rosse } \\
H \text {-Statistic }\end{array}$ & $\begin{array}{l}\ln (\mathrm{TI} / \mathrm{TA}) ; \\
\ln (\mathrm{II} / \mathrm{TA})\end{array}$ & $\ln \mathrm{TA}$ & Monopolistic Competition \\
\hline Jiang et al. (2004) & $\begin{array}{l}\text { 1992-2002, } \\
\text { Hong Kong }\end{array}$ & $\begin{array}{l}\text { Panzar-Rosse } \\
H \text {-Statistic }\end{array}$ & $\begin{array}{l}\text { (1) } \ln (\mathrm{TI} / \mathrm{TA}) \\
\text { (2) } \ln \mathrm{TI} /\end{array}$ & $\begin{array}{l}\text { (1) None } \\
\text { (2) ln TA }\end{array}$ & Perfect Competition \\
\hline Shaffer (2004b) & $\begin{array}{l}\text { 1984-1994, } \\
\text { U.S. Banks }\end{array}$ & $\begin{array}{l}\text { Panzar-Rosse } \\
H \text {-Statistic }\end{array}$ & $\ln \mathrm{TI}$ & $\ln \mathrm{TA}$ & Monopolistic Competition \\
\hline $\begin{array}{l}\text { Drakos and } \\
\text { Konstantinou } \\
(2005)\end{array}$ & $\begin{array}{l}\text { 1992-2000, } \\
\text { Former Soviet Union }\end{array}$ & $\begin{array}{l}\text { Panzar-Rosse } \\
H \text {-Statistic }\end{array}$ & $\ln \mathrm{TI}$ & $\ln \mathrm{TA}$ & Monopolistic Competition \\
\hline $\begin{array}{l}\text { Mkrtchyan } \\
(2005)\end{array}$ & 1998-2002, Armenia & $\begin{array}{l}\text { Panzar-Rosse } \\
H \text {-Statistic }\end{array}$ & $\ln$ II & $\ln \mathrm{TA}$ & Monopolistic Competition \\
\hline $\begin{array}{l}\text { Casu and } \\
\text { Girardone (2006) }\end{array}$ & 1997-2003, EU 15 & $\begin{array}{l}\text { Panzar-Rosse } \\
H \text {-Statistic }\end{array}$ & $\ln (\mathrm{TI} / \mathrm{TA})$ & $\ln \mathrm{TA}$ & Monopolistic Competition \\
\hline $\begin{array}{l}\text { Lee and Lee } \\
(2005)\end{array}$ & 1992-2002, Korea & $\begin{array}{l}\text { Panzar-Rosse } \\
H \text {-Statistic }\end{array}$ & $\begin{array}{l}\text { (1) } \ln (\mathrm{II} / \mathrm{TA}) \\
\text { (2) } \ln (\mathrm{TI} / \mathrm{TA}) \text {; } \\
\text { (3) } \ln \text { II; } \\
\text { (4) } \ln \text { TI }\end{array}$ & $\begin{array}{l}\text { (1) None; } \\
\text { (2) None; } \\
\text { (3) } \ln \mathrm{TA} \\
\text { (4) } \ln \mathrm{TA}\end{array}$ & Monopolistic Competition \\
\hline $\begin{array}{l}\text { Mamatzakis et al. } \\
(2005)\end{array}$ & $\begin{array}{l}\text { 1998-2002, } \\
\text { South-Eastern European } \\
\text { Countries }\end{array}$ & $\begin{array}{l}\text { Panzar-Rosse } \\
H \text {-Statistic }\end{array}$ & $\ln (\mathrm{TI} / \mathrm{TA})$ & & Monopolistic Competition \\
\hline $\begin{array}{l}\text { Al-Muharrami et } \\
\text { al. (2006) }\end{array}$ & $\begin{array}{l}\text { 1993-2002, Arab GCC } \\
\text { countries, }\end{array}$ & $\begin{array}{l}\text { Panzar-Rosse } \\
H \text {-Statistic }\end{array}$ & $\ln \mathrm{TI}$ & $\ln \mathrm{TA}$ & $\begin{array}{l}\text { Perfect Competition: Kuwait, Saudi Arabia, UAE; } \\
\text { Monopolistic Competition: Bahrian, Qatar, } \\
\text { Monopoly: Oman }\end{array}$ \\
\hline $\begin{array}{l}\text { Günalp and Çelik } \\
(2006)\end{array}$ & 1990-2000, Turkey & $\begin{array}{l}\text { Panzar-Rosse } \\
H \text {-Statistic }\end{array}$ & $\ln \mathrm{TI}$ & $\ln \mathrm{TA}$ & Monopolistic Competition \\
\hline $\begin{array}{l}\text { Staikouras and } \\
\text { Koutsomanoli - } \\
\text { Fillipaki (2006) }\end{array}$ & $\begin{array}{l}\text { 1998-2002, } \\
\text { EU } 10 \text { vs EU } 15\end{array}$ & $\begin{array}{l}\text { Panzar-Rosse } \\
H \text {-Statistic }\end{array}$ & $\ln (\mathrm{TI} / \mathrm{TA})$ & & Monopolistic Competition \\
\hline Yildirim and & 1993-2000, & Panzar-Rosse & $\ln (\mathrm{TI} / \mathrm{TA})$ & $\ln \mathrm{TA}$, & Monopolistic Competition \\
\hline
\end{tabular}


Table C.2. Empirical Evidence on the Use of Panzar-Rosse Model and Lerner Index (Chronologically)

\begin{tabular}{|c|c|c|c|c|c|}
\hline Author (Year) & Sample & $\begin{array}{l}\text { Financial } \\
\text { Indicators }\end{array}$ & $\begin{array}{l}\text { Dependent } \\
\text { Variable }\end{array}$ & Scaling & Key Findings \\
\hline $\begin{array}{l}\text { Philippatos } \\
(2007)\end{array}$ & $\begin{array}{l}11 \text { Latin American } \\
\text { Countries. }\end{array}$ & $H$-Statistic & & $\begin{array}{l}\ln \mathrm{EQ}, \\
\ln \mathrm{FA}\end{array}$ & \\
\hline $\begin{array}{l}\text { Bikker et al. } \\
(2007)\end{array}$ & $\begin{array}{l}1986-2005, \\
101 \text { countries, }\end{array}$ & $\begin{array}{l}\text { Panzar-Rosse } \\
H \text {-Statistic }\end{array}$ & $\begin{array}{l}\text { (1) } \ln \text { II; } \\
\text { (2) } \ln (\mathrm{II} / \mathrm{TA}) \\
\text { (3) } \ln \text { II } \\
\text { (4) } \ln \text { TI } \\
\text { (5) } \ln (\mathrm{TI} / \mathrm{TA}) \\
\text { (6) } \ln \mathrm{TI} \\
\end{array}$ & $\begin{array}{l}\text { (1) None; } \\
\text { (2) None; } \\
\text { (3) In TA } \\
\text { (4) None; } \\
\text { (5) None; } \\
\text { (6) In TA }\end{array}$ & \\
\hline $\begin{array}{l}\text { Matthews, } \\
\text { Murinde, and } \\
\text { Zhao (2007) }\end{array}$ & $\begin{array}{l}1980-2004, \\
12 \text { U.K. banks }\end{array}$ & $\begin{array}{l}\text { Panzar-Rosse } \\
H \text {-Statistic }\end{array}$ & $\begin{array}{l}\ln (\mathrm{TI} / \mathrm{TA}) \\
\ln (\mathrm{II} / \mathrm{TA})\end{array}$ & $\ln \mathrm{TA}$ & $\begin{array}{l}\text { The results confirm the consensus finding that competition in British } \\
\text { banking is most accurately characterized by the theoretical model of } \\
\text { monopolistic competition. There is evidence that the intensity of } \\
\text { competition in the core market for bank lending remained } \\
\text { approximately unchanged throughout the 1980s and 1990s. However, } \\
\text { competition appears to have become less intense in the non-core } \\
\text { (off-balance sheet) business of British banks. }\end{array}$ \\
\hline $\begin{array}{l}\text { Delis, Staikouras, } \\
\text { and Varlagas } \\
(2008)\end{array}$ & $\begin{array}{l}\text { 1993-2004, } \\
\text { Greece, Spain, } \\
\text { Latvia }\end{array}$ & $\begin{array}{l}\text { Panzar-Rosse } \\
H \text {-Statistic }\end{array}$ & $\ln \mathrm{TI}$ & & Monopolistic Competition \\
\hline $\begin{array}{l}\text { Lee and Nagano } \\
(2008)\end{array}$ & $\begin{array}{l}\text { 1993-2005, } \\
\text { Korea, Japan }\end{array}$ & $\begin{array}{l}\text { Panzar-Rosse } \\
H \text {-Statistic }\end{array}$ & $\ln (\mathrm{II} / \mathrm{TA})$ & & $\begin{array}{l}\text { High levels of banking market competitiveness are detected after } \\
\text { mergers in both the Korean and Japanese metropolitan areas. } \\
\text { Although the level of market competition remains low throughout } \\
\text { Japanese local cities, it is competitive compared with the metropolitan } \\
\text { area of Korea. This paper concludes that market concentration } \\
\text { brought about by bank mergers does not always result in low } \\
\text { competitiveness. }\end{array}$ \\
\hline $\begin{array}{l}\text { Carbó et al. } \\
(2009)\end{array}$ & $\begin{array}{l}\text { 1995-2001, } \\
14 \text { EU countries }\end{array}$ & $\begin{array}{l}\text { Panzar-Rosse } \\
H \text {-Statistic }\end{array}$ & $\ln \mathrm{TI}$ & $\ln \mathrm{TA}$ & \\
\hline Coccorese (2009) & 1998-2005, Italy & $\begin{array}{l}\text { Panzar-Rosse } \\
H \text {-Statistic }\end{array}$ & $\ln \mathrm{TI}$ & $\ln \mathrm{TA}$ & \\
\hline $\begin{array}{l}\text { Gischer and } \\
\text { Stiele (2009) }\end{array}$ & 1993-2002, Germany & $\begin{array}{l}\text { Panzar-Rosse } \\
H \text {-Statistic }\end{array}$ & $\ln$ II; & $\ln \mathrm{EQ}$ & Monopolistic Competition \\
\hline $\begin{array}{l}\text { Goddard and } \\
\text { Wilson (2009) }\end{array}$ & $\begin{array}{l}\text { 2001-2007, Canada, } \\
\text { France, Germany, } \\
\text { Italy, Japan, United } \\
\text { Kingdom, United States }\end{array}$ & $\begin{array}{l}\text { Panzar-Rosse } \\
H \text {-Statistic }\end{array}$ & $\begin{array}{l}\text { (1) } \ln \mathrm{II} \text {; } \\
\text { (2) } \ln \mathrm{TI}\end{array}$ & $\begin{array}{l}\text { (1) None, } \\
\text { (2) In TA }\end{array}$ & \\
\hline Schaeck et al. & 1980-2005, & Panzar-Rosse & $\ln (\mathrm{II} / \mathrm{TA})$ & & \\
\hline
\end{tabular}


Table C.2. Empirical Evidence on the Use of Panzar-Rosse Model and Lerner Index (Chronologically)

\begin{tabular}{|c|c|c|c|c|c|}
\hline Author (Year) & Sample & $\begin{array}{l}\text { Financial } \\
\text { Indicators }\end{array}$ & $\begin{array}{l}\text { Dependent } \\
\text { Variable }\end{array}$ & Scaling & Key Findings \\
\hline$(2009)$ & 45 countries & $H$-Statistic & & & \\
\hline $\begin{array}{l}\text { Turk-Ariss } \\
(2010)\end{array}$ & $\begin{array}{l}\text { 1999-2005, } 60 \\
\text { developing countries: } \\
\text { including Africa, } \\
\text { East/South } \\
\text { Asia and Pacific, } \\
\text { Eastern Europe and } \\
\text { Central } \\
\text { Asia, Latin America and } \\
\text { Caribbean, } \\
\text { and the Middle East. }\end{array}$ & $\begin{array}{l}\text { Lerner index } \\
\text { and } \\
\text { funding } \\
\text { adjusted } \\
\text { Lerner index }\end{array}$ & & & $\begin{array}{l}\text { The conventional Lerner figures show varying degrees of market } \\
\text { power across countries, but the figures are generally closely aligned } \\
\text { across all regions (around } 30 \% \text { price mark-up over marginal costs) } \\
\text { except for Latin America and the Caribbean where the conventional } \\
\text { Lerner is as low as } 17 \% \text {. The estimated efficiency and } \\
\text { funding-adjusted Lerner indices also vary across countries and } \\
\text { regions. }\end{array}$ \\
\hline $\begin{array}{l}\text { Olivero et al. } \\
(2011)\end{array}$ & $\begin{array}{l}\text { 1996-2006, } 10 \text { Asian } \\
\text { countries and } 10 \text { Latin } \\
\text { American countries }\end{array}$ & $\begin{array}{l}\text { Panzar-Rosse } \\
H \text {-Statistic }\end{array}$ & & & $\begin{array}{l}\text { Most estimates are positive and less than } 1 \text { indicates that banks in } \\
\text { Latin American and Asian countries seem to operate in a } \\
\text { monopolistically competitive environment. Exceptions include India, } \\
\text { Korea, and China from Asia and Venezuela from Latin America } \\
\text { which are shown to have negative values of the H statistics. This } \\
\text { implies a potential monopolistic environment or the presence of a } \\
\text { structural disequilibrium in their banking markets. Banking industries } \\
\text { in Latin America seem to be more competitive than those in Asia. }\end{array}$ \\
\hline $\begin{array}{l}\text { Stavarek and } \\
\text { Repkova (2011) }\end{array}$ & $\begin{array}{l}\text { 2001-2009, } \\
\text { Czech Republic }\end{array}$ & $\begin{array}{l}\text { Panzar-Rosse } \\
H \text {-Statistic }\end{array}$ & & & $\begin{array}{l}\text { Highly competitive market in period 2001-2005 and monopolistic } \\
\text { competition in 2005-2009. }\end{array}$ \\
\hline $\begin{array}{l}\text { Cipollini and } \\
\text { Fiordelisi (2012) }\end{array}$ & $\begin{array}{l}\text { 1996-2009, European } \\
\text { countries: Austria, } \\
\text { Belgium, Denmark, } \\
\text { Finland, } \\
\text { France, Germany, } \\
\text { Greece, } \\
\text { Ireland, Italy, } \\
\text { Netherlands, } \\
\text { Portugal, Spain, Sweden, } \\
\text { United Kingdom }\end{array}$ & Lerner index & & & $\begin{array}{l}\text { The mean value of the Lerner index suggests monopolistic } \\
\text { competition }\end{array}$ \\
\hline $\begin{array}{l}\text { Casu and } \\
\text { Girardone (2006) }\end{array}$ & $\begin{array}{l}\text { 2000-2005, European } \\
\text { countries: France, } \\
\text { Germany, Italy, Spain, } \\
\text { UK }\end{array}$ & Lerner index & & & $\begin{array}{l}\text { Values of both indices are diversified across time and across } \\
\text { countries, and suggest monopolistic competition. Spanish and Italian } \\
\text { banking industries seem to be the most competitive, with Lerner } \\
\text { index close to } 0 \text {. }\end{array}$ \\
\hline $\begin{array}{l}\text { Bikker et al. } \\
\text { (2012) }\end{array}$ & 1986-2004.67 countries & $\begin{array}{l}\text { Panzar-Rosse } \\
H \text {-Statistic }\end{array}$ & $\begin{array}{l}\text { (1) } \ln \text { II; } \\
\text { (2) } \ln (\mathrm{II} / \mathrm{TA})\end{array}$ & $\begin{array}{l}\text { (1) None; } \\
\text { (2) None; }\end{array}$ & \\
\hline
\end{tabular}


Table C.2. Empirical Evidence on the Use of Panzar-Rosse Model and Lerner Index (Chronologically)

\begin{tabular}{|c|c|c|c|c|c|}
\hline Author (Year) & Sample & $\begin{array}{l}\text { Financial } \\
\text { Indicators }\end{array}$ & $\begin{array}{l}\text { Dependent } \\
\text { Variable }\end{array}$ & Scaling & Key Findings \\
\hline & & & $\begin{array}{l}\text { (3) } \ln \text { II } \\
\text { (4) } \ln \text { TI } \\
\text { (5) } \ln (\mathrm{TI} / \mathrm{TA}) \\
\text { (6) } \ln \mathrm{TI} .\end{array}$ & $\begin{array}{l}\text { (3) } \ln \mathrm{TA} \\
\text { (4) None; } \\
\text { (5) None; } \\
\text { (6) } \ln \mathrm{TA}\end{array}$ & \\
\hline Beck et al. (2013) & 1994-2009, 79 countries & Lerner index & & & $\begin{array}{l}\text { The values of the index are positive and suggest monopolistic } \\
\text { competition }\end{array}$ \\
\hline Fu et al. (2014) & $\begin{array}{l}\text { 2003-2010, Asia Pacific } \\
\text { countries: } \\
\text { Australia, China, Hong } \\
\text { Kong, } \\
\text { India, Indonesia, Japan, } \\
\text { Korea, } \\
\text { Malaysia, Pakistan, } \\
\text { Philippines, Singapore, } \\
\text { Sri } \\
\text { Lanka, Taiwan, Thailand }\end{array}$ & $\begin{array}{l}\text { Lerner index } \\
\text { and } \\
\text { efficiency } \\
\text { adjusted } \\
\text { Lerner index }\end{array}$ & & & $\begin{array}{l}\text { Values of both indices are diversified across time and across } \\
\text { countries, and suggest monopolistic competition. The trend for the } \\
\text { Lerner index (non-structural measure) is descending between } 2005 \\
\text { and } 2008 \text { suggesting a decrease in pricing power. The Lerner index } \\
\text { exhibits varying degrees of market power across countries. Singapore } \\
\text { has the highest efficiency adjusted } \\
\text { Lerner index value }(0.44) \text {, whereas Taiwan has the lowest value } \\
(0.22) \text {. }\end{array}$ \\
\hline
\end{tabular}




\begin{tabular}{ll}
\hline Values of $H$ & Competitive Environment Test \\
\hline$H \leq 0$ & $\begin{array}{l}\text { Monopoly Equilibrium: each institution operates independently as under monopoly profit maximization } \\
\text { conditions }(H \text { is a decreasing function of the perceived demand elasticity }) \text { or perfect cartel }\end{array}$ \\
$0<H<1$ & Monopolistic competition: free entry equilibrium $(H$ is an increasing function of the perceived demand elasticity) \\
$H=1$ & Perfect competition: free entry equilibrium with full efficient capacity utilization
\end{tabular}




\title{
Appendix D: Chapter 4
}

Table D.1. Summaries of the Hypotheses and Findings in Chapters 1, 2, and 3.

Chapter (Title) Hypothesis

Main Findings

\begin{abstract}
Chapter 1: The Use of Financial Derivatives and Risks of U.S. Bank Holding Companies
Hypothesis 1.1: Financial derivatives impact (systematic interest rate, exchange rate and credit) risks of a $\mathrm{BHC}$.

Hypothesis 1.1a: Financial derivatives for hedging impact risks of a BHC.
\end{abstract}

Hypothesis 1.1b: Financial derivatives for trading impact risks of a BHC.

Hypothesis 1.2: The relationship between financial derivatives and risks is affected by a BHC's capital strength.

High capital reinforces the positive relationship between financial derivatives for trading and systematic risks, but weakens the positive relationship between financial derivatives for hedging and systematic risk.

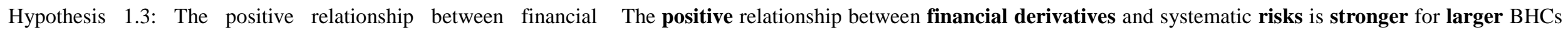
derivatives and risks intensifies for larger BHCs. (especially for Exchange rate derivatives and Credit derivatives)

Chapter 2: Quality of Bank Capital and Bank Lending Behavior during the Global Financial Crisis

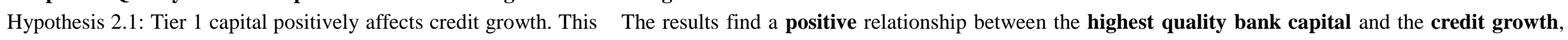
effect was more pronounced during the global financial crisis. and the interaction term constructed as a product of the tier 1 ratio and crisis dummy also demonstrates a positive relationship with loan growth. This supports the notion of tier 1 serving as a buffer and not an incentive mechanism for banks

Hypothesis 2.2: Tier 2 capital positively affects loan growth during normal times. During the global financial crisis, tier 2 capital negatively affected loan growth.

Hypothesis 2.3: The decline in bank lending during the global financial crisis was higher for banks with higher levels of interbank deposits and lower levels of customer deposits.

Hypothesis 2.4a: Higher market concentration (HHI) is associated with lower lending during normal times, but was associated with higher lending during the global financial crisis.

Hypothesis 2.4b: Higher tier 1 capital ratios of competing banks

We find some evidence that tier 2 positively affects lending growth in normal times. Contrary to Hypothesis 2.2, tier 2 capital had no significant effect on credit growth during the global financial crisis.

Customer deposits positively and significantly affected bank lending during the global financial crisis Interbank lending is positively associated with bank lending during normal times and negatively during the global financial crisis.

We observe a negative effect of the concentration index $\left(\mathrm{HHI}_{i, t-1}\right)$ on credit growth (although mostly insignificant) during normal times.

Competitors' tier 1 capital ratios have a significantly positive impact on loan growth during normal 
are positively associated with bank lending during normal times but were negatively associated during the global financial crisis.

Hypothesis 2.5a: Government owned banks sustained lending better during the global financial crisis than non-government owned banks.

Hypothesis 2.5b: Foreign ownership was associated with weaker lending during the global financial crisis.

Hypothesis 2.5c: The subsidiary status of banks was associated with stronger credit growth during the global financial crisis.

\section{Chapter 3: Competition in the Clearing and Settlement Industry}

Hypothesis 3.1: Competition between clearing and settlement institutions during the financial crises is higher than in normal time.

Hypothesis 3.2: ICSDs are exposed to the higher level of competition than CSDs.

Hypothesis 3.3: The size of clearing and settlement institutions positively affects the level of competition between clearing and settlement institutions. times, but this coefficient reversed during the global financial crisis. This provides support only for Hypothesis $2.4 \mathrm{~b}$.

We find a significant and positive effect of government ownership on credit growth in the global financial crisis. This corresponds to direct support of governments through ownership participation in banks and also confirms our Hypothesis $2.5 \mathrm{a}$.

We find some limited support for the negative effect of foreign ownership on lending growth during the global financial crisis. This is in line with Hypothesis $2.5 \mathrm{~b}$.

We find limited evidence that credit growth during the global financial crisis was affected by the organizational structure of the bank. That is, we find that a subsidiary bank cut back on lending less during the global financial crisis than a stand-alone entity. This is in line with Hypothesis $2.5 \mathrm{c}$.

The $\boldsymbol{H}$-statistic significantly increased during the global financial crisis.

The negative Boone indicator during the financial crisis is lower than in normal times,

Dummy variable of the global financial crisis is negatively and statistically significantly associated with Lerner index.

Competition between clearing and settlement institutions is higher during the financial crises period than in normal times.

The regression coefficient of the interaction terms between $\boldsymbol{H}$-statistics and ICSD is statistically significantly positive.

The negative coefficient of the interaction term between International CSDs and marginal cost indicates that the negative Boone indicator of International CSDs is lower than CSDs and international CSDs face higher competition than domestic CSDs.

ICSD is negatively and highly statistically significantly associated with Lerner index.

The regression coefficient of the interaction terms between $\boldsymbol{H}$-statistics and institution size is statistically significantly positive.

The result of Boone indicator shows the interaction term between institution size and marginal cost is negatively and statistically significantly associate with the profit of clearing and settlement institutions.

Lerner index is negatively related to the size of a clearing and settlement institution. Hence, larger institutions are exposed to higher competition. 
Table D.1. Summaries of the Hypotheses and Findings in Chapters 1, 2, and 3.

Chapter (Title) Hypothesis

Main Findings

Hypothesis 3.4: Mergers between CSDs are associated with higher competition between clearing and settlement institutions.

Hypothesis 3.5: Technological development increases competition between clearing and settlement institutions.

Hypothesis 3.6: Competition between clearing and settlement institutions in the U.S. market is higher than in the Europe.
The regression coefficient of the interaction term between the $\boldsymbol{H}$-statistics and dummy variable Merger is statistically significantly positive.

The interaction term between merger and marginal cost is negatively related to the profit, although it is insignificant, which indicates the negative Boone indicator of merged clearing and settlement institutions is lower than not merged institutions.

Merger is negatively but mostly insignificantly related to the Lerner index. This provides some but limited support that mergers between clearing and settlement institutions improve competition.

ICT ratio is statistically significantly positively associated with $\boldsymbol{H}$-statistic.

The ICT ratio has a negative and significant effect on Boone indicator and technological development increases competition between clearing and settlement institutions.

Variables ICT ratio and Time are negatively and significantly related to Lerner index.

We conclude that technological development increases competition between clearing and settlement institutions.

$\boldsymbol{H}$-statistic is higher in the U.S. market than in Europe.

Boone indicator of the clearing and settlement institutions in U.S. marker is lower than in the European market.

Dummy variable USregion is negatively and statistically significantly related to Lerner index.

These results indicate that competition between clearing and settlement institutions is higher in the U.S. market than in the European market. 


\section{Appendix E: Summary in Slovenian language / Daljši povzetek disertacije v slovenskem}

To poglavje se osredotoča na najpomembnejše ugotovitve disertacije. Glavni cilj pričujoče disertacije je ovrednotenje vloge izvedenih finančnih instrumentov, bančnega kapitala ter storitev obračuna in poravnave $v$ normalnih razmerah in med svetovno finančno krizo. Poglavje je sestavljeno iz štirih delov: najprej na kratko povzame ugotovitve vsakega poglavja disertacije in poda sistematični pregled ugotovitev, sledi obravnava glavnih vseobsegajočih teoretičnih in empiričnih prispevkov, na koncu pa še kratek zaključek disertacije.

\section{Povzetek najpomembnejših ugotovitev}

Prvo poglavje, ki temelji na razširjenem štirifaktorskem modelu, analizira razmerje med uporabo izvedenih finančnih instrumentov in sistematično izpostavljenostjo tveganju ameriških bančnih holdingov. Rezultati potrjujejo, da je uporaba izvedenih finančnih instrumentov bančnih holdingov povezana $\mathrm{z}$ večjo izpostavljenostjo sistematičnemu obrestnemu, tečajnemu in kreditnemu tveganju (t.i. 'nerazpršljivem' tveganju, ki se mu investitorji na finančnih trgih ne morejo izogniti). Zanimivo je to, da je pozitivno razmerje med izvedenimi finančnimi instrumenti in izpostavljenostjo sistematičnemu tveganju močnejše pri večjih bančnih holdingih kot pri manjših. Tako izvedeni finančni instrumenti za trgovanje kot tudi instrumenti za varovanje pred tveganjem so pozitivno in močno povezani z izpostavljenostjo bančnih holdingov sistematičnemu tveganju ( $\mathrm{v}$ primerih obrestnih, tečajnih in kreditnih izvedenih finančnih instrumentov). V času svetovne finančne krize so postala razmerja med obrestnimi in tečajnimi izvedenimi finančnimi instrumenti ter izpostavljenostjo sistematičnemu tveganju močnejša kot $\mathrm{v}$ normalnih razmerah, pozitivno razmerje med kreditnimi finančnimi instrumenti in sistematičnim kreditnim tveganjem pa manj izrazito.

Drugo poglavje preučuje, če in kako različne vrste bančnega kapitala vplivajo na bančna posojila. Analizira tudi vpliv velikosti bank in drugih razsežnosti, ki lahko vplivajo na upad kreditne rasti med finančno krizo. Rezultati kažejo na pozitiven vpliv deleža kapitala prvega reda na rast bančnih posojil med svetovno finančno krizo. Zdi se, da je vpliv bolj izrazit pri manjših bankah in bankah držav nečlanic OECD ter držav BRIK, prav tako pa so depoziti strank pozitivno vplivali na bančna posojila v času svetovne finančne krize. Poleg tega je delež kapitala drugega reda skupaj z medbančni vlogami pozitivno vplival na rast posojil v normalnih razmerah, v času svetovne krize pa so medbančne vloge negativno vplivale na rast bančnih posojil. Medtem ko so kapital prvega reda in depoziti strank predstavljali stabilen vir financiranja med svetovno finančno krizo, pa so kapital drugega reda in medbančne vloge spodbudile bančna posojila $\mathrm{V}$ normalnih razmerah, vendar ne tudi v času krize. V normalnih razmerah je bilo bančnih posojil več, če je bil delež kapitala prvega reda konkurenčnih bank visok, v času svetovne finančne krize pa so banke dajale več posojil, če je bil delež kapitala prvega reda konkurenčnih bank nizek. Vladno lastništvo je pomagalo bankam pri ohranjanju kreditne rasti med svetovno finančno krizo, učinek pa je bil statistično pomemben le v državah nečlanicah OECD in državah BRIK, ne pa tudi v državah članicah OECD. 
Tretje poglavje skuša analizirati konkurenčno okolje $\mathrm{v}$ industriji obračuna in poravnave. Natančneje, to poglavje uporablja model Panzar-Rosse (1982, 1987), Lernerjev indeks (1934) in Boonov indikator $(2001,2008)$ pri preučevanju konkurenčnih pogojev v industriji obračuna in poravnave ter pri preizkušanju, kako na konkurenco vpliva več dejavnikov. Empirični rezultati kažejo na obstoj monopolnega ravnovesja $\mathrm{v}$ industriji obračuna in poravnave. Model Panzar-Rosse, Boonov indikator in Lernerjev indeks potrjujejo naslednje sklepe: v času svetovne finančne krize je raven konkurence med institucijami obračuna in poravnave višja kot $\mathrm{v}$ normalnih razmerah. Mednarodne centralne depotne družbe (CDD) se soočajo $\mathrm{z}$ večjo konkurenco kot CDD na lokalnih trgih. Dokazi prav tako nakazujejo na to, da se konkurenca skozi čas nenehno povečuje, kar je mogoče pripisati tehnološkemu napredku in izvajanju novih sistemov obračuna in poravnave. Rezultati razkrivajo, da se konkurenca povečuje z velikostjo institucij obračuna in poravnave ter združitvami in prevzemi med le-temi. Ugotovitve tudi kažejo na to, da je konkurenca med institucijami obračuna in poravnave večja na ameriškem trgu kot na evropskem, kar pa kaže na to, da bo potrebno obnoviti pobudo za povečanje konkurence med evropskimi institucijami obračuna in poravnave.

Tabela 4.1 povzema raziskovalna vprašanja in glavne ugotovitve vsakega poglavja ter predstavi metodologije raziskave in prispevek k obstoječi literaturi.

\section{Vseobsegajoči teoretični in tmpirični prispevki}

Pomemben metodološki prispevek prvega poglavja disertacije je uporaba razširjenega modela Fame in Frencha (1992) in hkratna razmejitev sistematičnega ('nerazpršljivega') tveganja na tri komponente: sistematično obrestno, menjalno in kreditno tveganje. To nam omogoča, da skupno analiziramo vpliv obrestnih, menjalnih in kreditnih izvedenih finančnih instrumentov na ustrezna sistematična tveganja. Rezultati kažejo na to, da izvedeni finančni instrumenti pozitivno in močno vplivajo na izpostavljenost bančnih holdingov sistematičnemu tveganju. Večja uporaba obrestnih, menjalnih in kreditnih izvedenih finančnih instrumentov ustreza večjemu sistematičnemu obrestnemu, menjalnemu in kreditnemu tveganju. Sistematična tveganja pozitivno vplivajo tako na izvedene finančne instrumente za varovanje pred tveganjem, kot tudi na tiste za trgovanje.

$\mathrm{Na}$ razmerje med izvedenimi finančnimi instrumenti in sistematičnim tveganjem bančnih holdingov vpliva več dejavnikov. Finančni instrumenti izpostavljajo velike holdinge višji stopnji sistematičnega tveganja v primerjavi z malimi holdingi. Visok delež kapitala okrepi pozitivno razmerje med izvedenimi finančnimi instrumenti za trgovanje in sistematičnimi tveganji, a negativno vpliva na razmerje med finančnimi instrumenti za varovanje pred tveganjem in sistematičnim tveganjem. V času svetovne finančne krize je postalo razmerje med obrestnimi in menjalnimi izvedenimi finančnimi instrumenti ter izpostavljanju sistematičnemu tveganju močnejše kot v normalnih razmerah, pozitivno razmerje med kreditnimi izvedenimi finančnimi instrumenti in sistematičnim kreditnim tveganjem pa manj izrazito. 
Tabela 4.1. Povzetek Glavnih Ugotovitev in Prispevkov v Poglavjih 1, 2 in 3.

\begin{tabular}{|c|c|c|c|c|c|}
\hline Poglavje (naslov) & Vzorec & $\begin{array}{l}\text { Glavni vir } \\
\text { podatkov }\end{array}$ & Metodologija & Glavne ugotovitve & Prispevek \\
\hline $\begin{array}{l}\text { Poglavje 1: Uporaba } \\
\text { izvedenih finančnih } \\
\text { instrumentov in } \\
\text { tveganja ameriških } \\
\text { bančnih holdingov }\end{array}$ & $\begin{array}{l}\text { 1997-2012, } \\
\text { bančni } \\
\text { holdingi }\end{array}$ & $\begin{array}{l}\text { FR Y-9C; } \\
\text { CRSP; } \\
\text { Svet } \\
\text { guvernerjev } \\
\text { Centralne banke }\end{array}$ & $\begin{array}{l}\text { Model fiksnih } \\
\text { učinkov; } \\
\text { IV model; } \\
\text { GMM model. }\end{array}$ & $\begin{array}{l}\text { Izvedeni finančni instrumenti so pozitivno in močno povezani s } \\
\text { sistematičnim tveganjem bančnih holdingov. } \\
\text { Tako instrumenti za trgovanje kot tudi instrumenti za varovanje pred } \\
\text { tveganjem so pozitivno in močno povezani z izpostavljenostjo } \\
\text { sistematičnemu tveganju bančnih holdingov. } \\
\text { V času svetovne finančne krize so postala razmerja med obrestnimi in } \\
\text { tečajnimi izvedenimi finančnimi instrumenti ter izpostavljenostjo } \\
\text { sistematičnemu tveganju močnejša kot v normalnih razmerah, } \\
\text { pozitivno razmerje med kreditnimi instrumenti in sistematičnim } \\
\text { kreditnim tveganjem pa manj izrazito. }\end{array}$ & $\begin{array}{l}\text { Uporaba razširjenega } \\
\text { štirifaktorskega modela za } \\
\text { pridobitev dejavnikov } \\
\text { sistematičnega tveganja, } \\
\text { vključno s sistematičnim } \\
\text { kreditnim tveganjem; } \\
\text { diferenciacija izvedenih } \\
\text { finančnih instrumentov, ki se } \\
\text { uporabljajo za trgovanje kot } \\
\text { tudi za varovanje pred } \\
\text { tveganjem. }\end{array}$ \\
\hline $\begin{array}{l}\text { Poglavje 2: Kvaliteta } \\
\text { bančnega kapitala in } \\
\text { ravnanje bank s } \\
\text { posojili med } \\
\text { svetovno finančno } \\
\text { krizo }\end{array}$ & $\begin{array}{l}2000-2010, \\
\text { banke po } \\
\text { svetu }\end{array}$ & Bankscope & $\begin{array}{l}\text { Model fiksnih } \\
\text { učinkov; } \\
\text { Model IV; } \\
\text { Model GMM. }\end{array}$ & $\begin{array}{l}\text { Visoko kakovostni viri bančnega financiranja (t.j. bančni kapital } \\
\text { prvega reda in vloge na drobno) in prevladujoča vladna podpora so } \\
\text { bili ključnega pomena za neprekinjena bančna posojila med krizo. } \\
\text { Večja uporaba kapitala drugega reda in medbančnih depozitov bi } \\
\text { lahko bila pomembna pri povečanju posojil v normalnih razmerah, } \\
\text { kar pa ni podprlo aktivnosti dajanja posojil med krizo. }\end{array}$ & $\begin{array}{l}\text { Ločene ocene vpliva strukture } \\
\text { kapitala (deleža kapitala prvega } \\
\text { in drugega reda) na bančna } \\
\text { posojila v normalnih razmerah } \\
\text { in med svetovno finančno } \\
\text { krizo. }\end{array}$ \\
\hline $\begin{array}{l}\text { Poglavje 3: } \\
\text { Konkurenca v } \\
\text { industriji obračuna in } \\
\text { poravnave }\end{array}$ & $\begin{array}{l}\text { 1989-2012, } \\
\text { Institucije } \\
\text { obračuna in } \\
\text { poravnave }\end{array}$ & $\begin{array}{l}\text { Bankscope; } \\
\text { Letna poročila; } \\
\text { Svetovna banka. }\end{array}$ & $\begin{array}{l}\text { Model } \\
\text { Panzar-Rosse, } \\
\text { Lernerjev } \\
\text { indeks; } \\
\text { Boonov } \\
\text { indikator. }\end{array}$ & $\begin{array}{l}\text { Industrija obračuna in poravnave deluje pod pogoji monopolnega } \\
\text { ravnovesja. } \\
\text { Med svetovno finančno krizo je raven konkurence med institucijami } \\
\text { obračuna in poravnave višja kot v normalnih razmerah. Mednarodne } \\
\text { centralne depotne družbe (CDD) se soočajo z večjo konkurenco kot } \\
\text { CDD na lokalnih trgih. } \\
\text { Konkurenca se skozi čas nenehno povečuje, kar je mogoče pripisati } \\
\text { tehnološkemu napredku in izvajanju novih sistemov obračune in } \\
\text { poravnave. } \\
\text { Konkurenca se povečuje z velikostjo institucij obračuna in poravnave } \\
\text { ter združitvah in prevzemih med le-temi. } \\
\text { Konkurenca med institucijami obračuna in poravnave na ameriškem } \\
\text { trgu je znatno večja na kot na evropskem; }\end{array}$ & $\begin{array}{l}\text { To je prva obširna študija o } \\
\text { konkurenčnih pogojih } \mathrm{v} \\
\text { industriji obračuna in } \\
\text { poravnave; analiza dejavnikov, } \\
\text { ki vplivajo na konkurenco v } \\
\text { obračunu in poravnavai. }\end{array}$ \\
\hline
\end{tabular}


Prispevek drugega poglavja disertacije je vpogled v razlike med kapitalom prvega in drugega reda, depoziti strank in medbančnimi depoziti kot viri bančnega financiranja in ovrednotenje njihovega razmerja $\mathrm{z}$ bančnimi posojili. Rezultati kažejo, da višja kakovost banke, ki se financira $\mathrm{z}$ visokim deležem bančnega kapitala prvega reda in depozitov strank, ustrezneje podpira izdajo bančnih posojil $\mathrm{v}$ času krize. Nasprotno pa kapital drugega reda ne predstavlja ustrezne podpore za bančna posojila med finančno krizo.

Empirična analiza v drugem poglavju razkriva, da so dejavniki, ki vključujejo različne vrste depozitov in lastništev pomembni pri oblikovanju ravnanja bank s posojili v normalnih razmerah in med finančno krizo. Medbančni depoziti so negativno vplivali na bančna posojila med krizo, banke pa so to skušale nadomestiti z obračanjem na bolj stabilne vire financiranja, kot na primer vloge na drobno (Evropska centralna banka, 2011). Depoziti strank pa so medtem pozitivno vplivali na rast posojil med svetovno finančno krizo, kar nakazuje na to, da so bile vloge strank stabilne in so služile kot stabilen vir financiranja tudi med krizo. Poleg tega so bile banke v času svetovne finančne krize deležne široke podpore s strani vlad, kar jim je pomagalo reševati težave refinanciranja. To pa nakazuje na prednosti vladnega lastništva pri zmanjševanju kreditnega krča.

Prispevek tretjega poglavja te študije je sestavljen iz treh delov in vključuje pomemben doprinos k področju konkurenčne literature, s posebnim poudarkom na industriji obračuna in poravnave, medtem ko so se prejšnje študije osredotočale na ekonomije obsega, stroškovno učinkovitost in učinkovitost prihodkov ter tehnološkim napredkom $\mathrm{v}$ industriji obračuna in poravnave. Na primer, Schmiedel, Malkamaki in Tarkka (2006) se osredotočajo na dejavnike ekonomij obsega in na tehnološki razvoj. Van Cayseele in Wuyts (2007) ugotavljata, da ekonomije obsega obstajajo $\mathrm{v}$ evropskem obračunu in poravnavi, vendar še zdaleč ne pokrivajo celotnega evropskega trga. Disertacija analizira konkurenčne pogoje $v$ industriji obračuna in poravnave predvsem z uporabo modela Panzar-Rosse, Lernerjevega indeksa in Boonovega indikatorja pri preučevanju konkurence institucij obračuna in poravnave. Model Panzar-Rosse, Lernerjev indeks in Boonov indikator so v preteklosti v velikem obsegu uporabljali pri analizi narave konkurence $\mathrm{v}$ bančnih sistemih, vendar ne tudi pri analizi institucij obračuna in poravnave. Na koncu analiza primerja tudi konkurenco $v$ industriji obračuna in poravnave v ZDA in Evropi.

\section{Zaključek}

Disertacija analizira vlogo izvedenih finančnih instrumentov, bančnega kapitala (in drugih virov financiranja bank) ter dejavnosti obračuna in poravnave $\mathrm{v}$ normalnih razmerah in $\mathrm{v}$ času svetovne finančne krize. Disertacija prav tako analizira razmerje med uporabo izvedenih finančnih instrumentov in tveganj in raziskuje povezavo med kvaliteto bančnega kapitala in rastjo bančnih posojil. Poleg tega preučuje konkurenčne pogoje $\mathrm{v}$ industriji obračuna in poravnave in testira vpliv več dejavnikov na konkurenčne pogoje $\mathrm{v}$ industriji obračuna in poravnave.

Metodologije, ki so bile uporabljene $\mathrm{v}$ pričujoči analizi, zapolnjujejo pomembno vrzel v obstoječi literaturi in dajejo napreden vpogled v delovanje finančnih institucij, kar bo pomagalo 
upravnim skupnostim in oblikovalcem politike $\mathrm{k}$ boljšemu razumevanju delovanja le-teh. Z vidika upravljanja lahko finančne institucije izboljšajo učinkovitost z boljšim razumevanjem, na kakšen način izvedeni finančni instrumenti pripomorejo k izpostavljenosti tveganju, kako bi lahko odločitve o strukturi bančnega kapitala vplivale na kreditno rast in kako različni dejavniki vplivajo na konkurenčne pogoje v obračunu in poravnavi. 